\title{
Large eddy simulations of compressible magnetohydrodynamic turbulence
}

\author{
Dissertation \\ zur Erlangung des mathematisch-naturwissenschaftlichen Doktorgrades \\ "Doctor rerum naturalium" \\ der Georg-August-Universität Göttingen \\ im Promotionsprogramm PROPHYS \\ der Georg-August University School of Science (GAUSS)
}

vorgelegt von
Philipp Grete

aus Peine

Göttingen, 2016 
Betreuungsausschuss

Prof. Dr. Dominik Schleicher

Departamento de Astronomía, Universidad de Concepción, Chile

PD Dr. Wolfram Schmidt

Hamburger Sternwarte, Universität Hamburg, Germany

Prof. Dr. Laurent Gizon

Max-Planck-Institut für Sonnensystemforschung, Göttingen, Germany

Institut für Astrophysik, Georg-August-Universität Göttingen, Germany

Mitglieder der Prüfungskommision

Referent: PD Dr. Wolfram Schmidt

Hamburger Sternwarte, Universität Hamburg, Germany

Korreferent: Prof. Dr. Dr.h.c. Eberhard Bodenschatz

Max-Planck-Institut für Dynamik und Selbstorganisation, Göttingen, Germany

Institut für Nicht-Lineare Dynamik, Georg-August-Universität Göttingen, Germany

2. Korreferent: Prof. Dr. Marcus Brüggen

Hamburger Sternwarte, Universität Hamburg, Germany

Weitere Mitglieder der Prüfungskommission:

Prof. Dr. Dominik Schleicher

Departamento de Astronomía, Universidad de Concepción, Chile

Prof. Dr. Jens Niemeyer

Institut für Astrophysik, Georg-August-Universität Göttingen, Germany

Prof. Dr. Jörg Büchner

Max-Planck-Institut für Sonnensystemforschung, Göttingen, Germany

Prof. Dr. Andreas Dillmann

Institut für Aerodynamik und Strömungstechnik, DLR, Göttingen, Germany

Tag der mündlichen Prüfung: 9. September 2016 


\section{Contents}

\begin{tabular}{ll}
\hline Abstract & 5
\end{tabular}

\begin{tabular}{lll}
\hline & Introduction & 7
\end{tabular}

1.1 Motivation . . . . . . . . . . . . . . . . . 7

1.2 Turbulence . . . . . . . . . . . . . . . . . . . . . 9

1.2.1 Incompressible hydrodynamic turbulence . . . . . . . . . . . 9

1.2 .2 Compressibility and presence of magnetic fields . . . . . . . . . 11

1.2.3 Turbulence in astrophysics . . . . . . . . . . . . . . . . 13

1.3 Numerical methods . . . . . . . . . . . . . . . . . . . 15

1.3 .1 Overview . . . . . . . . . . . . . . . . 15

1.3 .2 High-resolution methods . . . . . . . . . . . . . . 17

1.4 Large eddy simulations $\ldots \ldots \ldots$. . . . . . . . . . . . . . . . . . . . 19

$1.4 .1 \quad$ Implicit LES . . . . . . . . . . . . . . . . . . . 21

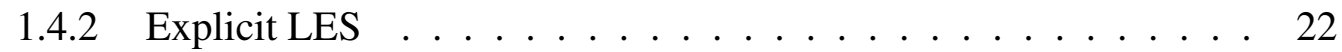

1.4 .3 Explicit discrete filters $\ldots \ldots \ldots . \ldots . \ldots 25$

1.4 .4 Verification and validation . . . . . . . . . . . 30

2 Paper I: Nonlinear closures for scale separation in supersonic MHD turbulence

3 Paper II: A nonlinear structural subgrid-scale closure for compressible MHD. I. Derivation and energy dissipation properties

4 Paper III: A nonlinear structural subgrid-scale closure for compressible MHD. II. A priori comparison on turbulence simulation data

5 Paper IV: Comparative statistics of selected subgrid-scale models in large eddy simulations of decaying, supersonic MHD turbulence 71

\begin{tabular}{|ll|}
6 & Summary and conclusions \\
\hline
\end{tabular}

\begin{tabular}{ll}
\hline Bibliography & 91
\end{tabular}

\begin{tabular}{|ll}
\hline A Discrete filter approximations & 101
\end{tabular}

\begin{tabular}{ll}
\hline Scientific contributions & 103
\end{tabular}

\begin{tabular}{ll}
\hline Acknowledgements & 105
\end{tabular} 



\section{Abstract}

Supersonic, magnetohydrodynamic (MHD) turbulence is thought to play an important role in many processes - especially in astrophysics, where detailed three-dimensional observations are scarce. Simulations can partially fill this gap and help to understand these processes. However, direct simulations with realistic parameters are often not feasible. Consequently, large eddy simulations (LES) have emerged as a viable alternative. In LES the overall complexity is reduced by simulating only large and intermediate scales directly. The smallest scales, usually referred to as subgrid-scales (SGS), are introduced to the simulation by means of an SGS model. Thus, the overall quality of an LES with respect to properly accounting for small-scale physics crucially depends on the quality of the SGS model. While there has been a lot of successful research on SGS models in the hydrodynamic regime for decades, SGS modeling in MHD is a rather recent topic, in particular, in the compressible regime.

In this thesis, we derive and validate a new nonlinear MHD SGS model that explicitly takes compressibility effects into account. A filter is used to separate the large and intermediate scales, and it is thought to mimic finite resolution effects. In the derivation, we use a deconvolution approach on the filter kernel. With this approach, we are able to derive nonlinear closures for all SGS terms in MHD: the turbulent Reynolds and Maxwell stresses, and the turbulent electromotive force (EMF). We validate the new closures both a priori and a posteriori.

In the a priori tests, we use high-resolution reference data of stationary, homogeneous, isotropic MHD turbulence to compare exact SGS quantities against predictions by the closures. The comparison includes, for example, correlations of turbulent fluxes, the average dissipative behavior, and alignment of SGS vectors such as the EMF. In order to quantify the performance of the new nonlinear closure, this comparison is conducted from the subsonic (sonic Mach number $\left.\mathrm{M}_{\mathrm{s}} \approx 0.2\right)$ to the highly supersonic $\left(\mathrm{M}_{\mathrm{s}} \approx 20\right)$ regime, and against other SGS closures. The latter include established closures of eddy-viscosity and scale-similarity type. In all tests and over the entire parameter space, we find that the proposed closures are (significantly) closer to the reference data than the other closures.

In the a posteriori tests, we perform large eddy simulations of decaying, supersonic MHD turbulence with initial $\mathrm{M}_{\mathrm{s}} \approx 3$. We implemented closures of all types, i.e. of eddy-viscosity, scale-similarity and nonlinear type, as an SGS model and evaluated their performance in comparison to simulations without a model (and at higher resolution). We find that the models need to be calculated on a scale larger than the grid scale, e.g. by an explicit filter, to have an influence on the dynamics at all. Furthermore, we show that only the proposed nonlinear closure improves higher-order statistics. 



\section{Introduction}

\subsection{Motivation}

Turbulence accompanies everyone - for some people more unconscious, for example, if they rely on turbulent mixing by stirring their cup of coffee with sugar and milk, than for others, who consciously study turbulence. In general, a turbulent flow may be understood as a qualitative description of a system that is dominated by virtually random, chaotic motions with a huge number of excited modes and intermittent features. This behavior makes exact predictions on the evolution of the flow extremely challenging (if not impossible). The difficulty stems from the nonlinearity of the fundamental equations, e.g. the $(\boldsymbol{u} \cdot \nabla) \boldsymbol{u}$ term in the incompressible $(\nabla \cdot \boldsymbol{u}=0)$ Navier-Stokes equations

$$
\frac{\partial \boldsymbol{u}}{\partial t}+(\boldsymbol{u} \cdot \nabla) \boldsymbol{u}=-\frac{1}{\rho} \nabla P+v \nabla^{2} \boldsymbol{u}
$$

with velocity $\boldsymbol{u}$, density $\rho$, pressure $P$ and dynamic viscosity $v$. While exact solutions only exists for a few specific cases, flows can often be described statistically or in an even more simplified qualitative manner. One important qualitative description is the (hydrodynamic) Reynolds number

$$
\operatorname{Re}=\frac{L V}{v}
$$

which combines a characteristic lengthscale $L$ and velocity $V$ with $v$ to give a dimensionless number, see e.g. textbooks of Frisch (1995), Pope (2000) and Davidson (2004). This Reynolds number is often used to characterize flows given that flows with similar Reynolds number have similar properties. Illustratively, this is the reason why e.g. the real air flow over an airplane wing can be imitated with a model of reduced size that is subject to a flow of higher velocity. Typically, a flow is considered turbulent at Reynolds number of $O\left(10^{3}\right)$, which is already reached for the initially mentioned cup of coffee under stirring of one revolution per second.

While an exact dynamical description of a cup of coffee is barely possible, the situation is astrophysics is even worse. Not only is the dynamical range naturally larger by many orders of magnitudes, but also compressibility and the presence of magnetic fields introduce further complexity. Here, the dynamics for an electrically conducting fluid can be described by the magnetohydrodynamic (MHD) equations, which can be derived by combining Maxwell's equations with the Navier-Stokes equations. The basic equations 
of compressible ideal MHD are given by

$$
\begin{aligned}
\frac{\partial \rho}{\partial t}+\nabla \cdot(\rho \boldsymbol{u}) & =0, \\
\frac{\partial \rho \boldsymbol{u}}{\partial t}+\nabla \cdot(\rho \boldsymbol{u} \otimes \boldsymbol{u}-\boldsymbol{B} \otimes \boldsymbol{B})+\nabla\left(P+\frac{B^{2}}{2}\right) & =\mathbf{0}, \\
\frac{\partial \boldsymbol{B}}{\partial t}-\nabla \times(\boldsymbol{u} \times \boldsymbol{B}) & =\mathbf{0} .
\end{aligned}
$$

with the magnetic field $\boldsymbol{B}$ incorporating units of $1 / \sqrt{4 \pi}$. Even though they look rather simple, they provide the key to a plethora of phenomena and processes on vastly varying scales. These include, for example, the geo-dynamo, which is responsible for maintaining the Earth's protective magnetic field, the strong collimation of astrophysical jets by magnetic fields, and the magnetorotational instability, which regulates angular momentum transport in accretion disks. A more detailed description of selected turbulent astrophysical systems including their characteristic quantities is given in in subsection 1.2.3. All these processes have in common large Reynolds numbers and the exact impact and importance of turbulence is still subject to active research in most processes.

The lack of final answers to many questions is partially due to the lack of detailed observations. Naturally, most astrophysical observations provide 2-dimensional information only and at a limited resolution. Here, numerical simulations can complement observations as they provide not only a detailed control over the environment, but also allow for the analysis for full (instantaneous) 3-dimensional data. Unfortunately, direct numerical simulations covering realistic regimes, i.e. the $\mathrm{Re}>O\left(10^{9}\right)$, are not going to be possible for quite some time. Even the largest purely hydrodynamic turbulence simulations reaches only $\operatorname{Re} \sim O\left(10^{4}\right)$ (Federrath et al. 2016). Thus, alternative approaches that reduce the complexity while still maintaining the fundamental physics are required. One approach is the so-called large eddy simulation (LES). The justification and application of this specific type of simulation to ideal, compressible MHD turbulence is the subject of this thesis.

The thesis is structured as follows. In the remainder of the introduction, the phenomenology and basic equations of turbulence are first discussed in the following subsection - starting with incompressible hydrodynamic turbulence and then extended to the most important features of compressible MHD turbulence. Afterwards, turbulence in astrophysics on different scales and in different regimes is presented in more detail. Then, in section 1.3 the standard numerical methods for computational (magneto-) fluid dynamics are introduced as the concept of large eddy simulation is intrinsically linked to the numerics. LES themselves are then presented in section 1.4 including the general formalism, the distinction between implicit and explicit LES, (discrete) filters, and different verification and validation methods. The first results for a simple nonlinear closure are given in chapter 2 . This closure is then extended to explicitly account for compressibility from a theoretical point of view in chapter 3 , and tested against most common alternative closures a priori in chapter 4 . Afterwards, the testing is extended to a posteriori application in simulations of decaying MHD turbulence in chapter 5. Finally, in chapter 6 all results are summarized and discussed in a broader context. 


\subsection{Turbulence}

\subsubsection{Incompressible hydrodynamic turbulence}

To date, there is no universal theory of turbulence. However, in certain areas, not only phenomenological descriptions exists, but also analytical ones. The most famous example is probably Kolmogorov (1941) with his theory of homogeneous, isotropic, incompressible hydrodynamic turbulence.

While the phenomenology has already been described earlier, e.g. by Richardson (1922) "Big whirls have little whirls that feed on their velocity, and little whirls have lesser whirls and so on to viscosity.", Kolmogorov first derived an analytical description. This energy cascade, which is mediated by interacting whirls (or eddies), is a characteristic feature of turbulence. In addition to homogeneity and isotropy, Kolmogorov also assumed stationarity of the flow, and that the energy dissipation is finite even in the case of $\operatorname{Re} \rightarrow \infty$ (i.e. viscosity $v \rightarrow 0$ ). Even though the underlying theory is more complicated, see e.g. Frisch (1995) for further details, the key results can be illustrated by simple arguments and dimensional analysis (Choudhuri 1998).

Assuming that the largest eddies have characteristic velocity $V$ and size $L$, which is close to the scale where energy is injected into the stationary system, they are characterized by

$$
\operatorname{Re} \sim \frac{L V}{v} .
$$

Eddies at these scales now break up into eddies of smaller and smaller scale until molecular dissipation starts to be important at $\mathrm{Re} \approx 1$. Thus, at the smallest scales with eddies of size $l_{\mathrm{d}}$ and velocity $v_{\mathrm{d}}$

$$
l_{\mathrm{d}} v_{\mathrm{d}} \sim v .
$$

At the intermediate scales $l_{\mathrm{d}}<l<L$ energy is simply transferred down-scale at the dissipation rate $\varepsilon$, which, under the assumption of being independent of $v$, is given by dimensional analysis as

$$
\varepsilon \sim \frac{v^{3}}{l} .
$$

With this equation two important relations can be derived. First, given that (1.6) holds for both the smallest and largest eddies in stationary turbulence, it can be combined with (1.4) and (1.5) to obtain a scaling relation that describes the scale separation for a given large scale Reynolds number:

$$
\frac{L}{l_{d}} \sim \operatorname{Re}^{3 / 4} .
$$

Second, the energy spectrum $E(k)$ can be assumed to depend only on the constant dissipation rate and the wavenumber $k$. Again, dimensional analysis results in

$$
E(k) \sim \varepsilon^{2 / 3} k^{-5 / 3} .
$$

This famous Kolmogorov slope of $-5 / 3$ is illustrated in figure 1.1 . The range of scales where this relation holds is usually referred to as inertial (sub)range and was observed in nature, in experiments and in numerical simulations. 


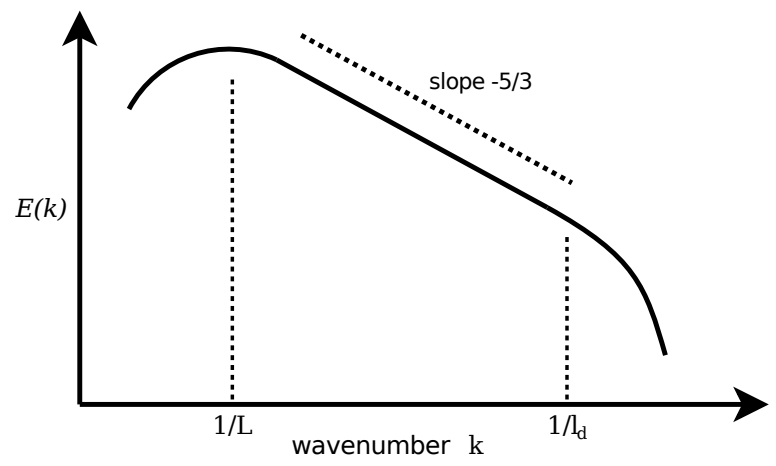

Figure 1.1: Illustration of the Kolmogorov energy spectrum.

Structure functions are an important statistical measure to describe turbulence, especially as the second order longitudinal structure function is directly related to the energy spectrum, see e.g. Pope (2000) for comprehensive introduction, which is roughly followed here. In general, structure functions of order $p$ are defined as

$$
S_{\|}^{p}(l)=\left\langle|(\boldsymbol{u}(\boldsymbol{x}+\boldsymbol{l})-\boldsymbol{u}(\boldsymbol{x})) \cdot \boldsymbol{l} / l|^{p}\right\rangle .
$$

In this formulation, they are the moments of the instantaneous velocity $\boldsymbol{u}$ differences in the direction of the spatial separation $\boldsymbol{l}$ in the homogeneous, isotropic case. The following scaling relation

$$
S_{\|}^{p}(l) \propto l^{\zeta p}
$$

is obtained if the characteristic velocity $v$ in $(1.6)$ is interpreted as the velocity increments at that scale. Here, $\zeta_{p}=p / 3$ is the structure function exponent.

While it has been observed that these scaling prediction are quite accurate at lower orders $(p \lesssim 3)$, there are significant deviations at higher orders (Anselmet et al. 1984). The deviations stem from the oversimplified hypothesis of self-similarity over all inertial scales in the original derivation by Kolmogorov. In fact, as later recognized by Kolmogorov (1962) and Obukhov (1962), the phenomenon of intermittency invalidates the hypothesis. Not the mean dissipation over all scales should be used in (1.6), but the mean value corresponding to the separation $l$. This is usually referred to as refined self-similarity hypothesis and requires an additional parameter specifying intermittency. Nowadays, a further refinement by She and Leveque (1994) is often used. It does not require the additional parameter and has great success in correctly predicting scaling exponents with

$$
\zeta_{p}=\frac{p}{9}+2\left(1-\left(\frac{2}{3}\right)^{p / 3}\right)
$$

Here, the main assumption is that dissipation does not occur isotropically, but most strongly in intermittent structures of filamentary nature.

Extended self-similarity (ESS) was discovered almost at the same time by Benzi et al. (1993) and also used to confirm the predicted scaling exponents (1.11). Then, as now, not all systems (including experiments and numerical simulations) showed a clear inertial 
range in the energy spectrum for several reasons, such as insufficient spatial separation between the largest and smallest scales, or too much numerical dissipation (see following section 1.3 for a discussion). This lack of a clear inertial range is equivalent to a lack of the corresponding scaling range in the structure functions. Nevertheless, it is observed that the individual structure functions of different order $p$ are self-similar in the sense of suffering the same defects. Thus, Benzi et al. (1993) showed that, if plotted against each other, the relative structure functions recover a power law scaling with a corresponding relative scaling exponent $Z_{p}=\zeta_{p} / \zeta_{p, \text { ref }}$. In addition to this, the range of scales over which this scaling is present extends far beyond the original (potentially negligible) inertial range. Eventually, the original scaling exponents can be recovered by this procedure if $S_{\|}^{3}(l)$ is used as reference structure function. Given that $\zeta_{3}=1$, the absolute and relative exponents are identical $Z_{p}=\zeta_{p} / \zeta_{3}=\zeta_{p}$. This concept also extends to MHD as later shown, for example, in figure $5 \mathrm{c}$ of chapter 5 .

\subsubsection{Compressibility and presence of magnetic fields}

The previous discussion of turbulence covered important aspects of incompressible hydrodynamics. Here, in a similar fashion important aspects of turbulence are presented that include compressible, i.e. $\nabla \cdot \boldsymbol{u} \neq 0$, effects and/or magnetic fields, i.e. $\boldsymbol{B} \neq \mathbf{0}$. For additional information, see e.g. the textbooks of Biskamp (2003) and Choudhuri (1998) or more recent reviews by Tobias et al. (2013) and Miesch et al. (2015), which are the basis of this subsection.

First of all, compressible fluids support waves. Several linear waves exist which are valid for small initial perturbations. On the one hand, there are acoustic or sound waves in compressible hydrodynamics. Here, the pressure provides a restoring force for waves that propagate at the speed of sound $c_{\mathrm{s}}$. One the other hand, if magnetic fields are present, additional restoring forces allow for additional wave types. First, the Alfvén wave is a transversal wave propagating at the Alfvén velocity $v_{\mathrm{a}}=B / \sqrt{4 \pi \rho}$ in the direction of $\boldsymbol{B}$. Magnetic tension provides the restoring force, and given that Alfvén waves are incompressible waves, they also exists in incompressible MHD. Second, magnetosonic waves are compressible waves and can be further discriminated in slow and fast modes. The former is characterized by the thermal and magnetic pressure working against each other as restoring forces, while in the case of the fast mode both pressures act together. The exact behavior of these modes is more complex and depends on the angle between the wave vector and the magnetic field, and the ratio of sound to Alfvén speed.

Besides these nondispersive linear waves, there also exist nonlinear waves, which more strongly influence the compressible turbulence dynamics. Here, even smooth initial conditions can develop a steep profile - a thin region in which the fluid variables change rapidly, i.e. make a jump. This is also referred to (and mathematically treated as) a discontinuity. Using the hydrodynamic equations in conservational form, jump conditions can be derived to relate the up- and downstream fluid quantities over this region. These Rankine-Huginiot conditions can then be treated as a Riemann problem to solve the behavior over the discontinuity. For instance, a contact discontinuity is associated with a jump in the density but otherwise continuous transition of pressure or velocity. In contrast to this, a shock wave, which builds if the velocity is faster than the local speed of sound, is associated with a jump in all fluid quantities. In general, the width (or sharpness) of the 
discontinuity is only limited by the dissipative properties of the system, e.g. viscosity in HD. Similar considerations also apply to MHD. Again, the additional degrees-of-freedom allow for additional complexity. For example, the magnetic field is bent away from the shock-normal for a fast-mode MHD shock, while it is changed in the direction of the shock-normal for a slow-mode MHD shock.

In general, compressibility effects are important once the root mean square sonic Mach number reaches $M_{s} \gtrsim 0.3$. In turbulence, shock waves themselves are important as dissipation in a shock-dominated system predominately occurs at the spatially sparse shock fronts. More specifically, dissipation does not occur in quasi-one-dimensional vortex filaments any more, but rather in structures of higher dimension. These structures include shock fronts and, in the case of MHD, reconnection sites. They are usually not volume filling and occupy only a small fraction of the available volume. In the remaining volume dissipation is usually small.

While this is mostly attributed to the compressibility and thus also applies in HD, there are two characteristics of MHD turbulence that are fundamentally different from hydrodynamic turbulence: increasing anisotropy on the smallest scales and nonlocal spectral transfer.

The former stems from the fact that a background magnetic field does not vanish at any scale. In contrast to hydrodynamics, where, for example, large scale rotation gets increasingly negligible at decreasing scales so that isotropy can be assumed for scales smaller than a given scale, the influence of a background magnetic field in MHD actually increases with decreasing scales. In addition to this, this background field does not necessarily need to be some (potentially) external (mean) field. A sufficiently smooth field on a certain scale will again look like a large scale mean field on scales sufficiently smaller. This anisotropy is important as it provides preferred directions for physical processes such as thermal conduction or dissipation (Braginskii 1965).

The nonlocal spectral transfer is especially relevant in the case of turbulence with finite magnetic helicity. In hydrodynamics, the two ideal invariants, energy and kinetic helicity, have a direct cascade, i.e. their ideal spectrum (cf. the absolute equilibrium distribution) peaks at high wavenumbers $k_{0}$ and any energy injected at scale larger than $k$ relaxes towards $k_{0}$. Moreover, this relaxation is local in the sense that energy is transferred preferentially between wavenumber of similar magnitude, which is why the process is typically referred to as a cascade. The ideal MHD equations have three ideal invariants: energy, cross-helicity and magnetic helicity. Again, the ideal spectrum of the first two quantities is peaked at high $k$. However, the spectrum of the magnetic helicity is peaked at low $k$. For this reason, it relaxes towards the largest scales allowed in a system. In addition, the link between the different relaxation processes allows for nonlocal transfer.

Finally, there are two interesting extreme regimes in MHD turbulence: systems with a strong background field and quasi-isotropic turbulence.

On the one hand, situations with a strong (potentially external) mean or background magnetic field can (at least in the incompressible limit) be described by interacting Alfvén waves. For this reason, this regime is also referred to as Alfvénic turbulence. Here, the anisotropy of MHD turbulence is well pronounced. The initial description by Iroshnikov (1964) and Kraichnan (1965) based on the assumption of weak interactions between waves on all scales, is now superseded by Goldreich and Sridhar (1995) (GS). While the former derived a scaling of the energy spectrum of $E(k) \propto k^{-3 / 2}$, the latter derived a Kol- 
mogorov scaling for the parallel cascade $E(k) \propto k^{-5 / 3}$. The major change in the GS theory is that interactions below a certain scale are no longer weak, but actually strong.

On the other hand, quasi-isotropic turbulence with no external field (or of negligible strength) is of particular importance for astrophysics as it, for example, allows for the generation of magnetic fields (from weak seed fields) and maintenance against dissipative processes (Brandenburg and Subramanian 2005). In the case of the so called small-scale dynamo, a stretch-twist-fold mechanism (figuratively) converts kinetic energy of turbulent small-scale motion into magnetic energy, e.g. described by the Kazantsev (1968) model. This growth of magnetic field strength first ends once the magnetic energy reaches comparable levels to the kinetic energy at the driving scale, i.e. magnetic field works against the turbulent driving. This back-reaction from magnetic fields, which are amplified on a certain scale, onto motions at a different scale is another example of nonlocal interactions in MHD turbulence.

In general, different regimes are typically characterized by dimensionless numbers. Analogous to the kinetic Reynolds number, a magnetic Reynolds Rm can be defined, which is a relative measure of magnetic field advection versus diffusion by the finite resistivity

$$
\mathrm{Rm}=\frac{L V}{\eta}
$$

with magnetic diffusivity $\eta$. Combining both, kinetic and magnetic Reynolds numbers yield another dimensionless number. The magnetic Prandtl number

$$
\operatorname{Pm}=\frac{\operatorname{Rm}}{\operatorname{Re}}=\frac{v}{\eta}
$$

provides an indication about which microscopic process dominates the MHD flow (assuming diffusion is relevant in the first place). Finally, the plasma beta

$$
\beta_{\mathrm{p}}=\frac{2 P}{B^{2}}
$$

denotes the ratio between thermal and magnetic pressure.

\subsubsection{Turbulence in astrophysics}

Turbulence plays an important role in many astrophysical processes and on many different scales. Even when microphysical proceses are excluded, phenomena on scales ranging over several orders or magnitudes, e.g. from stellar scales to galaxy cluster scales, are influenced or dominated by turbulent processes.

Turbulence in stars One example in relatively close proximity to Earth is the convection zone of the Sun (Canuto and Christensen-Dalsgaard 1998, Miesch 2005). All stars with masses of $\sim 1 M_{\odot}$ are thought to consist of a core, which is surrounded by a radiative and a convection zone. The names stem from the dominant process that transport the energy released from fusion in the core to the outside, i.e. radiative transfer in the radiation zone and convective transport in the convection zone. The largest convection cells in the 
Sun have characteristic sizes of $L \sim 200 \mathrm{Mm}$ with velocities $V \sim 100 \mathrm{~m} / \mathrm{s}$, and the associated viscosity is estimated to be $v \sim 1 \mathrm{~cm}^{2} / \mathrm{s}$. Thus, the Reynolds number is $\operatorname{Re} \sim 10^{14}$ indicating a highly turbulent flow.

Moreover, most of the material in the convection zone is a fully ionized hydrogen plasma, which is, in turn, susceptible to magnetic fields. The magnetic Reynolds number is also very large $\mathrm{Rm} \sim 10^{9}$ (given magnetic diffusivities of $\eta \sim 10^{5} \mathrm{~cm}^{2} / \mathrm{s}$ ) suggesting a tangled magnetic field driven by the turbulent velocity field.

In addition to this, the flow velocity approaches the speed of sound in the near surface layers of the convection zone. Hence, compressibility effects are getting important. In particular, the turbulent hydrodynamic pressure is thought to contribute a substantial fraction to the total pressure. However, as recently shown by Brandenburg et al. (2011), the presence of turbulent magnetic fields (at high Reynolds numbers) can effectively reduce the total turbulent pressure again. This negative effective magnetic pressure instability (NEMPI) is currently discussed as driver for active regions atop the convection zone.

One of the most pronounced features in observations of the Sun is, for instance, the characteristic granulation pattern due to the dynamics in the turbulent convection zone. Furthermore, the magnetic field of the Sun is thought to be generated at the bottom of the convection zone. Thus, in order to understand the global magnetic field dynamics of the Sun, e.g. the 22-year solar cycle, turbulence in the convection zone needs to be understood.

Turbulence in the interstellar medium (ISM) The ISM is a very dynamic environment due to being subject to self-gravity, compressibility, thermal processes, magnetic fields, and turbulence (Vázquez-Semadeni 2015, Falgarone et al. 2015, Klessen and Glover 2016). Altogether the ISM combines $\lesssim 1 \%$ of the total mass in the Milky Way and consists of multiple phases. The latter are typically separated into molecular clouds (temperatures of $O(10 \mathrm{~K}))$, a cold $(50-100 \mathrm{~K})$ and warm $(6000-10000 \mathrm{~K})$ neutral component, and a warm $(\sim 8000 \mathrm{~K})$ and hot $\left(\sim 10^{6} \mathrm{~K}\right)$ ionized component. While the characteristic velocities in the ionized components are sub- to transonic, observations of the neutral components and molecular clouds reveal supersonic motions.

Molecular clouds are often of special interest given that they are the regions where star formation takes place. With velocity dispersions of $\sigma \sim 1 \mathrm{~km} / \mathrm{s}$ at length scales of $1 \mathrm{pc}$ and dynamic viscosities $v \sim 10^{16} \mathrm{~cm}^{2} / \mathrm{s}$ their kinetic Reynolds number is $\operatorname{Re} \sim 10^{8}$. Thus, molecular clouds are highly turbulent with sonic Mach numbers $\gtrsim 10 \mathrm{M}_{\mathrm{s}}$ indicating strong compressibility. In addition, the observed magnetic fields strengths at these length scales are $\sim 10^{-5} \mathrm{G}$ yielding super-Alfvénic velocities.

In the past, coherent magnetic fields were thought to provide sufficient support against the collapse of the gravitationally unstable molecular clouds (Shu et al. 1987). However, more recently, this picture changed to a more dynamic one. For instance, Federrath (2015) shows that (close to) realistic star formation rates can be reproduced if and only if compressible turbulence, magnetic fields and stellar feedback are considered simultaneously. The rates are well below what is theoretically expected from a purely gravitational collapse. Hence, a correct treatment of compressible, magnetized turbulence is important.

Turbulence in clusters of galaxies Galaxy clusters are the largest (several Mpc), most massive gravitationally bound objects known, with typical masses of $10^{14}-10^{15} M_{\odot}$ 
(Brunetti and Jones 2015, Brüggen and Vazza 2015). According to the standard model, the vast majority of their total mass is dark matter. The second largest contribution (and the dominant baryonic one) with $\sim 10 \%$ of the total mass is the intracluster medium (ICM). With temperatures of $10^{7}-10^{8} \mathrm{~K}$ and number densities of $n \sim 10^{-1}-10^{-4} \mathrm{~cm}^{-3}$, the ICM is a weakly collisional, hot, dilute plasma with $\beta_{\mathrm{p}} \gg 1$.

Again, turbulence and magnetic fields have been observed (or suggested from observations) as in the previous two examples. However, the regime is quite different. For instance, the low collisionality (cf. long mean free path) allows for charged particles to be significantly affected by the presence of (even weak) magnetic fields. As a result, the overall flow can be susceptible to plasma (i.e. non-MHD) instabilities, and the flow is subject to anisotropic processes such as thermal conduction, which is suppressed in the direction perpendicular to the magnetic field. Moreover, the total (kinetic and magnetic) turbulent pressure contributes $\sim 10 \%$ to the total thermal pressure.

Nevertheless, the turbulent magnetic field in cluster cores with a characteristic lengthscale of $\sim 10 \mathrm{kpc}$ and strength of a few $\mu \mathrm{G}$ is dynamically unimportant on larger scales. This field strength translates to super-Alfénic $\left(\mathrm{M}_{\mathrm{a}} \sim 5-10\right)$ motion in the core, while the characteristic flow velocities are generally subsonic $\left(\mathrm{M}_{\mathrm{s}} \sim 0.25-0.6\right)$. Still, supersonic shocks $\left(\mathrm{M}_{\mathrm{s}} \sim 2-3\right)$ are observed, which are thought to be driven from cluster mergers. Thus, as an approximation the ICM has been described by hydrodynamic turbulence with mixed solenoidal and compressive modes in the past.

More recently, Egan et al. (2016) analyzed several cosmological MHD simulations of galaxy clusters with respect to statistical properties of turbulence and magnetic fields. On the one hand, they find that the ICM as a whole can be characterized by isotropic, magnetized turbulence. On the other hand, there is no global trend with respect to anisotropy of the magnetic field in the cluster sample. For this reason, a single global model is insufficient and a more local treatment of anisotropies is required. In addition, magnetic field amplification by the small-scale dynamo, which is physically expected (Subramanian et al.2006), and the magnetic field autocorrelation length, which is naturally linked to the process, are limited by resolution. Thus, a correct (physical) treatment of local and small-scale processes is important - especially as galaxy clusters are used to determine cosmological parameters, and serve as a testbed of plasma physics in an extreme environment.

\subsection{Numerical methods}

\subsubsection{Overview}

After the presentation of the governing equations in analytical form in the previous section 1.1, the necessary steps to solve these with the help of a computer are given here. The field is generally referred to as computational fluid dynamics (CFD). While there is a wealth of methods available with certain advantages and disadvantages each, here, only a brief overview of the methods is given with more details on methods common in compressible (magneto-)hydrodynamics. For more information see the general textbooks of Ferziger and Perić (2002) or the more in-depth books on hyperbolic conservation laws by Toro (2009) and LeVeque (2002). 
The first step in any method is the discretization of the differential equations. On the one hand, space needs to be discretized. In general, one differentiates between structured and unstructured grids. For structured grids the spatial topology is fixed. Each discrete point in space has fixed neighbours in space and this structure does not evolve in time. This eases the implementation as discrete stencils (and the underlying coordinate system) need to be defined only once and can then be used throughout the simulations, which is also one of the main disadvantages of this approach. Due to the fixed layout, a dynamic in the sense of temporally evolving spatial resolution can only be achieved by introducing additional (more complex) methods such as adaptive mesh refinement (Berger and Colella 1989).

On the other hand, the continuous differential operators need to be replaced by discrete approximations. Again, several different approaches are common in literature.

Finite differences FD methods are traditionally used due to their simplicity, both with respect to construction and implementation. Flow quantities are discretized on the respective grid points. The discretized differential operators can easily be derived by e.g. Taylor expansion and ultimately only depend on a limited number of adjacent points. The main disadvantages of this method are that it provides incorrect jump conditions for shocks and that it is not conservative. The latter is generally unfavorable for a conservative system such as periodic boxes governed by the (magneto-)hydrodynamic equations, which are commonly used in astrophysics or turbulence research.

Finite volume FV methods follow a different approach to alleviate this problem. The general idea is to recast the differential equations into conservative form and calculate the fluxes through boundary surfaces of a control volume. Hence, the method is conservative by construction. However, another problem is introduced by this approach in its basic formulation. Given that fluid quantities are not defined at individual grid points any more, but by the average volume density within a control volume, their spatial distribution within the cells needs to reconstructed first in order to determine the interface states. Here, spurious oscillations can be introduced to the simulation violating, for example, monotonicity, if no special care is taken. Godunov (1959) realized this deficiency and proved that linear, monotonicity preserving schemes can only achieve first order accuracy. Based on this reasoning higher-order, nonlinear methods have been developed afterwards, which are conservative and capable of properly handling shocks. For this reason, they are commonly used for compressible turbulence simulations and more details about these methods are given in the following subsection.

For completeness, three other approaches in CFD should be mentioned, which are fundamentally different in certain aspects from the previously described methods. They are much less often used in systems dominated by highly compressible dynamics for different reasons.

Spectral methods These methods (Canuto et al. 1988) have been applied with great success in the regime of incompressible turbulence where the solutions are smooth, e.g. a superposition of solenoidal motion. This smoothness allows for the easy transformation of the governing equations to spectral space (and back), where differentiation reduces to 
simple multiplication. This corresponds to the evolution of a global solution rather than the local (in physical space) solution at discrete points (or over discrete volumes). However, this global aspect also limits this method to simple geometries (such as a periodic equidistant grid) in addition to requiring smooth solutions.

Finite element FE methods can be seen as a link between spectral and finite volume methods. The idea of discretizing space in volumetric flow elements is borrowed from the latter approach. However, each element is approximated by a local polynomial and weighted with a specific function, which can be understood as a spectral method with local basis functions. The overall solution is then evolved by minimizing the weight of the specific functions. While this approach is very flexible, e.g. with respect to geometry, other aspects are less straightforward. However, the extension to compressible, discontinuous flows is a rather recent area of research and their broader application to complex flows as typically found in astrophysics is less developed.

Smoothed particle hydrodynamics Contrary to the previously described methods SPH is completely meshfree and the equations are not solved based on their Eulerian form, but from a Lagrangian point of view. Thus, fluid quantities such as density are not evolved within an external frame of reference, but the fluid itself is represented by individual fluid parcels that evolve freely with the flow. The evolution of and interaction between particles are then calculated by means of a smoothing function or kernel which spreads the fluid quantities in space. While the basic concept and implementation is straightforward and has the advantage of being locally adaptive with respect to the spatial resolution by construction, its accuracy is highly dependent on the smoothing kernel. The latter is also responsible for less frequent use of SPH in supersonic, shock-dominated turbulent flows because (at least in the original formulation) discontinuities and small-scale dynamics are artificially smeared out below the characteristic smoothing length of the kernel. Nevertheless, the method has successfully been applied to astrophysical problems in the past, for example, for cosmological simulations with the well-known SPH code GADGET by Springel (2005).

\subsubsection{High-resolution methods}

In the early days of CFD it was recognized that the most straightforward schemes, e.g. based on finite central difference stencils, are unconditionally unstable. This led to the development of so called upwind methods, which employ one-sided finite differences in the upwind direction. While these methods are conditionally stable, they resulted in firstorder accurate schemes only. In addition to this, the local upwind direction needs to be determined. This is straightforward in linear advection problems, but generally not possible in the nonlinear case. Moreover, several characteristics exists as solutions to the hyperbolic system of equations (cf. method of characteristics). Along these characteristics the hyperbolic system can be described by ordinary differential equations.

On this basis, Godunov (1959) introduced a nonlinear upwind finite volume method (often simply referred to as Godunov method) that can be characterized by a three step reconstruct-evolve-average (REA) algorithm. 
First, in the reconstruction step the spatial structure within a cell is reconstructed from the volume averages in that specific cell and neighbouring ones by piecewise polynomials. At first order the local average is taken constant everywhere in the cell. With increasing order reconstruction can be done piecewise linear (PLM), piecewise parabolic (PPM), etc. However, special care needs to be taken when performing this reconstruction so that no new minima or maxima are introduced, which would result in nonphysical oscillations at discontinuities. This procedure is referred to as slope limiting and many different slope limiters with different properties exist in literature. As discussed later in the context of implicit large eddy simulation (subsection 1.4.1) the combination of reconstruction and slope limiting inherently controls the numerical dissipation of a scheme.

Second, the reconstructed states are evolved in time. The main idea of the Godunov method is to evolve the solution along the individual characteristic and not as a whole. For this reason, a Riemann problem is locally solved on all interfaces between cells in order to determine the correct flux. While the Riemann problem can be solved exactly even though, through iterative methods only - most often approximate Riemann solvers are used in this step. Different approximate Riemann solver are usually discriminated by the amount and types of the characteristics that are followed. In hydrodynamics three characteristics exist: a shock wave, a contact discontinuity and a rarefaction wave. One of the most famous and widely distributed Riemann solvers is probably the Harten-Lax-vanLeer (HLL) solver, which captures two waves. A more accurate solver is, for example is HLLC ("C" for Contact), which captures one additional wave for a better resolution of intermediate waves (Toro 2009) and is also also frequently used. In MHD, the additional governing equations allow for additional characteristics corresponding to Alfvén waves, and fast and slow magnetosonic waves. While the hydrodynamic Riemann solvers can also be applied in MHD (missing of course depending on the problem more or less important features), extensions specifically capturing MHD characteristics have been proposed as well. These include, for example, HLLD ("D" for Discontinuities), which is designed to capture fast shocks (Miyoshi and Kusano 2005).

The third and final step of the REA cycle is the spatial averaging over the intermediate solution at the end of the timestep. However, this integration is not done explicitly, but automatically accounted for by the total flux through all interfaces.

From these basic ingredients higher-resolution methods can be derived. Here, the basic concept is to use a high-accuracy method (and thus a less numerically dissipative one) in smooth regions of the flow and employ appropriate (potentially first order) upwind methods at discontinuities. Ideally, these schemes are automatically locally adaptive and thus referred to as shock-capturing schemes. A well-known class of these schemes are total variation diminishing (TVD) schemes, whose main goal is that the total variation of the numerical solution does not increase over time. A famous representative of this kind in the Monotone Upstream-centred Scheme for Conservation Laws (MUSCL) scheme that is implemented in many codes, e.g. Enzo (Bryan et al.|2014) and FLash (Fryxell et al.|2000). In general, these schemes are nowadays quite mature, but cannot be extended to higher orders as a whole in straightforward fashion. Alternative approaches, e.g. essentially nonoscillatory (ENO) and weighted ENO (WENO) gained more and more attention recently (Balsara et al.2009).

Finally, in the context of MHD another constraint further complicates the development of numerical schemes: $\nabla \cdot \boldsymbol{B}=0$. In order to not have any magnetic monopoles, this 
constraint is ideally fulfilled to machine precision. Two mechanism are frequently employed. On the one hand, the constrained transport Evans and Hawley (1988) scheme is capable of keeping $\nabla \cdot \boldsymbol{B}=0$ exactly (to machine precision) by evolving not the magnetic field, but a potential (e.g. the electric) field. This potential field is typically defined on the edges of interfaces so that the magnetic field can be calculated readily by Stokes theorem. On the other hand, Dedner et al. (2002) introduced a method of hyperbolic divergence cleaning. In this scheme, the magnetic field is evolved along with the other fluid quantities potentially allowing for $\nabla \cdot \boldsymbol{B} \neq 0$ at the end of a timestep. Figuratively, these $\nabla \cdot \boldsymbol{B} \neq 0$ are collected and evolved by an additional equation corresponding to a divergence wave. Eventually, this divergence wave is then advecting any $\nabla \cdot \boldsymbol{B} \neq 0$ away from its source while simultaneously damping it down. Moreover, the hyperbolicity and conservational properties of the system are retained. Tests by Wang and Abel (2009) have shown that the $\nabla \cdot \boldsymbol{B} \neq 0$ present throughout the simulations are dynamically not important.

\subsection{Large eddy simulations}

One of the main challenges in computational (magneto-)hydrodynamics is the high nonlinearity of the basic equations. Simulations with physically realistic parameters - especially in the realm of astrophysics - are rarely possible. For example, even in the simplest case of incompressible hydrodynamics, the resources required to resolve a 3-dimensional simulation scale with $\mathrm{Re}^{3}$ (cf. equation (1.7) for three spatial and one temporal dimension (Davidson 2004)) Due to the many different scales involved in astrophysics, Reynolds numbers are easily getting very large, for example reaching $\operatorname{Re} \sim 10^{14}$ in the solar convection zone as illustrated in subsection 1.2.3. Thus, direct numerical simulation (DNS) that resolve all scales are often not possible.

While there are different approaches to solve this problem, e.g. by more or less motivated simplifications of the equations or the physical system of interest, one particular approach has been successfully used for decades: large eddy simulations (LES). The main idea of LES is based on the observation that in many systems most of the dynamicsgoverning energy is contained on the largest scales (cf. for example the turbulence energy spectrum Figure 1.1). These largest scales vary spatially - by construction - the least and usually develop on the largest timescales $T \propto L$. For this reason, they are also the computationally least expensive ones in the system.

The general idea of large eddy simulation is simple: the computationally cheap large and intermediate scales are simulated directly, whereas the smallest scales are modeled. From a practical point of view, these small scales are associated with processes occuring on scales that are typically below the scale of the discrete grid. Thus, the corresponding models are referred to as subgrid-scale (SGS) models. For a general introduction in the realm of hydrodynamics see Sagaut (2006) (incompressible), Garnier et al. (2009) (compressible) or Schmidt (2015) (astrophysical). For MHD there are recent (to the author's knowledge, the first) reviews by Chernyshov et al. (2014) and Miesch et al. (2015).

Formally, the separation of scales is achieved by the application of a low-pass filter to the dynamical equations, i.e. the convolution of a quantity $\phi$ with a filter kernel $G$ : 
$\bar{\phi}=G * \phi$. In the case of the ideal MHD equations (1.3) the filtered equations read

$$
\begin{aligned}
\frac{\partial \bar{\rho}}{\partial t}+\nabla \cdot(\bar{\rho} \widetilde{\boldsymbol{u}}) & =0, \\
\frac{\partial \bar{\rho} \widetilde{\boldsymbol{u}}}{\partial t}+\nabla \cdot(\bar{\rho} \widetilde{\boldsymbol{u}} \otimes \widetilde{\boldsymbol{u}}-\overline{\boldsymbol{B}} \otimes \overline{\boldsymbol{B}})+\nabla\left(\bar{P}+\frac{\bar{B}^{2}}{2}\right) & =-\nabla \cdot \tau, \\
\frac{\partial \overline{\boldsymbol{B}}}{\partial t}-\nabla \times(\widetilde{\boldsymbol{u}} \times \overline{\boldsymbol{B}}) & =\nabla \times \mathcal{E} .
\end{aligned}
$$

for a static, homogeneous and isotropic filter under periodic boundary conditions. A more detailed treatment of the requirements and properties of different (discrete) filters follows in subsection 1.4.3. Normal filtered quantities, such as the filtered density $\bar{\rho}$, magnetic field $\bar{B}$ and pressure $\bar{P}$, are denoted by an overbar, $\bar{\square}$. The tilde, $\widetilde{\square}$, indicates massweighted filtering, e.g. for the velocity field $\widetilde{\boldsymbol{u}}=\overline{\rho \boldsymbol{u}} / \bar{\rho}$. It is also referred to as Favre (1983) filtering who first introduced it to LES for two reasons. First, in the context of LES, filtered individual quantities are considered resolved and thus readily available in the simulation. However, the finite volume methods usually employed in the supersonic regime do not evolve the velocity field directly, but evolve the conserved momentum field, which effectively corresponds to an implicit mass-weighting. Second, by this change of variables, no additional terms, e.g. in the mass-conservation equation (1.15a), enter the equations.

The overall form of the filtered equations 1.15 is identical to the unfiltered ones, but for two new terms: the SGS stress $\tau$ in the momentum equation, and the turbulent electromotive force $(\mathrm{EMF}) \mathcal{E}$ in the induction equation. Analytically, they are expressed as

$$
\begin{aligned}
\tau_{i j} & =\tau_{i j}^{\mathrm{u}}-\tau_{i j}^{\mathrm{b}}+\left(\overline{B^{2}}-\bar{B}^{2}\right) \frac{\delta_{i j}}{2} \quad \text { and } \\
\mathcal{E} & =\overline{\boldsymbol{u} \times \boldsymbol{B}}-\widetilde{\boldsymbol{u}} \times \overline{\boldsymbol{B}}
\end{aligned}
$$

where the stress tensor is further decomposed into the turbulent (or SGS) Reynolds stress $\tau_{i j}^{\mathrm{u}}$ and the SGS Maxwell stress $\tau_{i j}^{\mathrm{b}}$ with

$$
\tau_{i j}^{\mathrm{u}} \equiv \bar{\rho}\left(\widetilde{u_{i} u_{j}}-\widetilde{u}_{i} \widetilde{u}_{j}\right) \text { and } \tau_{i j}^{\mathrm{b}} \equiv\left(\overline{B_{i} B_{j}}-\bar{B}_{i} \bar{B}_{j}\right)
$$

However, these new terms require closure as only filtered individual quantities are thought to be known, but not filtered mixed quantities such as $\widetilde{u_{i} u_{j}}, \overline{B_{i} B_{j}}$, and $\overline{\boldsymbol{u} \times \boldsymbol{B}}$. Different closure strategies are discussed in the following two subsections.

In general, these terms incorporate the dynamics across and below the filter (or grid) scale. This is an important distinction to the Reynolds-averaged Navier-Stokes (RANS) formalism, where interactions between resolved and unresolved scales are absent in the the turbulent stresses. The stresses in RANS incorporate dynamics below the filter scale only.

Finally, there is another important point in the compressible MHD LES formalism, which clearly separates it from the incompressible HD LES formalism: the subgrid-scale 
energies. Their definitions can readily be derived from the filtered total energy

$$
\bar{E}=\underbrace{\frac{1}{2} \widetilde{\rho u}^{2}+\frac{1}{2} \bar{B}^{2}}_{\text {(resolved) }}+\underbrace{\frac{1}{2} \bar{\rho}\left(\widetilde{u^{2}}-\widetilde{u}^{2}\right)+\frac{1}{2}\left(\overline{B^{2}}-\bar{B}^{2}\right)}_{=E_{\mathrm{sgs}}^{\mathrm{u}}+E_{\mathrm{sgg}}^{\mathrm{b}}=E_{\mathrm{sgs}}(\text { unresolved })},
$$

which can be decomposed into its resolved and the unresolved, SGS parts, $E_{\mathrm{sgs}}^{\mathrm{u}}$ and $E_{\mathrm{sgs}}^{\mathrm{b}}$. In hydrodynamics, there is only kinetic SGS energy and it is equivalent to the turbulent pressure. While in the incompressible regime it is usually deemed to be small and either neglected or absorbed into a "modified" pressure, its contribution are not negligible any more in the compressible regime Schmidt and Federrath (2011). Moreover, in the realm of MHD there is not only kinetic SGS energy, but also magnetic SGS energy (proportional to the turbulent magnetic pressure). Comparing equations (1.19) and (1.18) it is clearly visibly that the SGS energies are given by the traces of the SGS stresses by virtue of the identity

$$
\tau_{k k}^{\square}=2 E_{\mathrm{sgs}}^{\square}
$$

This is later important for so-called zero-equations closures, i.e. closures that do not require additional dynamical equations, in particular, for the SGS energies. General closure strategies are now presented in the next two subsections.

\subsubsection{Implicit LES}

The main idea behind implicit large eddy simulations (ILES) is that the SGS terms ( $\tau$ and $\mathcal{E}$ ) are not explicitly closed by an (external) model, but rather by the numerical discretization itself. In other words, the grid provides the filtering operation $\bar{\square}$. For a detailed presentation see the collection by Grinstein et al. (2007) and in particular the chapter 5 by Margolin and Rider (2007) therein, which is followed here.

They use the modified equation analysis (MEA), which was originally developed to analyze the stability of numerical algorithms, to asses the nature of the truncation error in finite-volume schemes. Based on a Taylor series expansion about the grid spacing $\Delta_{x}$ of the discrete terms, i.e. the governing equations already in discretized form, the modified equation of a FV scheme can be written as

$$
\frac{\partial \boldsymbol{U}}{\partial t}+\nabla \cdot \boldsymbol{F}(\boldsymbol{U})=\nabla \cdot \mathcal{T}\left(\boldsymbol{U}, \Delta_{x}, \Delta_{t}\right),
$$

with a vector of conserved quantities $\boldsymbol{U}$, a general flux function $\boldsymbol{F}$ and the truncation error written in the form of the divergence of an SGS term $\mathcal{T}$. When applied to highresolution FV method with cell-centered quantities, this term can further be separated into a term attributed to inertial transport $\mathcal{T}_{\text {hyp }}$, and a dissipative term $\mathcal{T}_{\text {diss }}$, which effectively suppresses oscillations.

If a piecewise constant reconstruction is used (cf. subsection 1.3.2), MEA shows that the overall algorithm is only first order accurate with the dissipative error $\nabla \cdot \mathcal{T}_{\text {diss }} \sim \Delta_{x}$ dominating over the inertial error $\nabla \cdot \mathcal{T}_{\text {hyp }} \sim \Delta_{x}^{2}$. This does not yield a high-resolution method. A high-resolution method can be constructed from higher-order reconstruction and nonlinear slope limiting to prevent nonphysical oscillations. In general, a slope limiter 
chooses (or interpolates) between the left and right hand side differences of the (volume) averaged quantities, i.e. it is a function of the form $\varphi\left(U_{j}-U_{j-1}, U_{j+1}-U_{j}\right)$. For example, applying MEA to a scheme consisting of piecewise linear reconstruction with a minmod (taking smallest absolute value) slope limiter

$$
\varphi_{\mathrm{mm}}(a, b)= \begin{cases}\operatorname{minmod}(a, b), & \text { if } a b>0, \\ 0, & \text { if } a b \leq 0,\end{cases}
$$

results in the following implicit SGS contributions at leading order:

$$
\mathcal{T}_{\text {hyp }}=-\Delta_{x}^{2}\left(\frac{1}{24} \frac{\partial^{2} F\left(\partial_{x} U\right)^{2}}{\partial U^{2}}+\frac{1}{12} \frac{\partial F\left(\partial_{x x} U\right)}{\partial U}\right) \quad \text { and } \quad \mathcal{T}_{\text {diss }}=\frac{\Delta_{x}^{2}}{4} \frac{\left|\partial_{U} F\left(\partial_{x} U \partial_{x x} U\right)\right|}{\partial_{x} U}
$$

Thus, the scheme is formally of second order. However, dissipation and inertial transport errors are of the same order, which is unfavorable in ILES with respect to establishing a distinct inertial range.

A simple modification can resolve this deficiency. Performing the analysis again with a double minmod limiter

$$
\varphi_{\mathrm{dmm}}(a, b)= \begin{cases}\operatorname{minmod}\left(\frac{a+b}{2}, 2 a, 2 b\right), & \text { if } a b>0, \\ 0, & \text { if } a b \leq 0,\end{cases}
$$

gives a similar $\mathcal{T}_{\text {hyp }}$ term, but the $\mathcal{T}_{\text {diss }}$ term is now of $O\left(\Delta_{x}^{3}\right)$ at leading order

$$
\mathcal{T}_{\text {diss }}=\frac{\Delta_{x}^{3}}{8}\left|\frac{\partial F\left(\partial_{x x x} U\right)}{\partial U}\right| \text {. }
$$

With this limiter it is expected that the inertial range is less affected by numerical dissipation and should thus be more pronounced (or extended).

This analysis of the implicit SGS contributions exemplifies the crucial aspect of the numerical scheme (and the slope limiter in particular) with respect to SGS model properties and the overall quality of the solution. Moreover, the different error terms can be related to different (explicit) SGS model, e.g. regarding their intrinsic properties, as shown in the following subsection.

\subsubsection{Explicit LES}

In contrast to ILES, which can be understood as discretizing the primary equations first and then interpreting the result as an implicit filter operation, explicit large eddy simulation can be seen as applying the filter operation first and then discretizing the resulting equations - including the new SGS terms or rather their closures.

In general, there are two different approaches to close the SGS stress tensor $\tau(1.16)$ and electromotive force $\mathcal{E}$ (1.17): functional and structural modeling. In the following, the general ideas (Sagaut 2006, Garnier et al. 2009) behind the the closures used in this thesis are introduced and an overview of the most important features can be found in table 1.1. 
Table 1.1: Overview of the different SGS model families used in the thesis.

\begin{tabular}{|c|c|c|c|}
\hline & Eddy-viscosity $^{a}$ & Scale-similarity $^{a}$ & Nonlinear \\
\hline Closure type & Functional & Structural & Structural \\
\hline SGS Reynolds stress & $-2 \bar{\rho} v^{\mathrm{u}} \widetilde{\mathcal{S}}_{i j}^{*}$ & $\overline{\bar{\rho}}\left({\widetilde{\widetilde{u_{i}}}}_{\widetilde{u}_{j}}-\widetilde{\widetilde{u_{i}}} \widetilde{\widetilde{u}_{j}}\right)$ & $\frac{1}{12} \Delta^{2} \widetilde{\rho}_{i, k} \widetilde{u}_{j, k}$ \\
\hline SGS Maxwell stress & $-2 v^{\mathrm{b}} \overline{\mathcal{M}}_{i j}$ & $\overline{B_{i} B_{j}}-\overline{\overline{B_{i}}} \overline{\overline{B_{j}}}$ & $\frac{1}{12} \Delta^{2} \bar{B}_{i, k} \bar{B}_{j, k}$ \\
\hline Electromotive force & $-\eta_{t} \overline{\mathbf{J}}$ & $\underbrace{}_{\widetilde{\boldsymbol{u}} \times \bar{B}}-\underbrace{\widetilde{B}}_{\widetilde{\boldsymbol{u}}}$ & $\begin{array}{l}\frac{1}{12} \Delta^{2} \varepsilon_{i j k}\left[\widetilde{u}_{j, l} \bar{B}_{k, l}\right. \\
\left.\quad-(\ln \bar{\rho})_{, l} \widetilde{u}_{j, l} \bar{B}_{k}\right]\end{array}$ \\
\hline Primary motivation & $\begin{array}{l}\text { Turbulence is of } \\
\text { dissipative nature }\end{array}$ & $\begin{array}{l}\text { Turbulence } \\
\text { dynamics is } \\
\text { self-similar }\end{array}$ & $\begin{array}{l}\text { Approximate } \\
\text { deconvolution of } \\
\text { the filter kernel }\end{array}$ \\
\hline
\end{tabular}

Functional modeling The oldest approach to modeling aims at reproducing a certain effect on the resolved scales from the unresolved or non-represented scales. The most famous representative of this approach is the eddy-viscosity model (also Smagorinsky (1963) model, who introduced the concept to LES). In analogy to molecular viscosity, a similar term with a turbulent eddy-viscosity $v_{k}$ proportional to the kinetic rate-of-strain tensor $\mathcal{S}_{i j}=\left(u_{i, j}+u_{j, i}\right) / 2$ is introduced as follows.

$$
\tau_{i j}^{\mathrm{u} *} \propto-2 \bar{\rho} v^{\mathrm{u}} \widetilde{\mathcal{S}}_{i j}^{*}
$$

The superscript star denotes the traceless part of a tensor, i.e. $\square_{i j}^{*}=\square_{i j}-\delta_{i j} \square_{k k} / 3$. Thus, an additional closure for the trace (cf. the kinetic SGS energy) is required if turbulent pressure should explicitly be accounted for. It is based on the assumption that the main function of turbulence is energy dissipation. This is straightforwardly exemplified given that the turbulent energy flux $\Sigma^{E}=\tau_{i j} \widetilde{\mathcal{S}}_{i j}$, which encodes the transfer of energy between resolved and unresolved scales, is of definite sign.

Several scalings have been proposed to determine the local strength of the turbulent viscosity $v_{k}$ based on different physical considerations. For instance, Vreman et al. (1994) use the turbulent energy as a proxy whereas Müller and Carati (2002) use the turbulent cross-helicity. The latter is motivated by including the interaction between velocity and magnetic field in the case of MHD turbulence. In general, the concept of functional closures has already been transferred to MHD earlier. Yoshizawa (1990), for example, introduces - in analogy to the eddy-viscosity for the SGS Reynolds stress - an eddydiffusivity $v^{\mathrm{b}}$ for the SGS Maxwell stress, which is proportional to the magnetic rate-ofstrain tensor $\mathcal{M}_{i j}=\left(B_{i, j}+B_{j, i}\right) / 2$ as shown in table 1.1. Moreover, he proposes a closure for the electromotive force that consists of three components to incorporate a) unresolved helicity effects, b) turbulent resistivity and c) dynamo action due SGS cross-helicity. The full model has been further studied in more detail by Miki and Menon (2008) and Yokoi 
(2013), whereas the turbulent resistivity $\eta_{t}$ alone, which is proportional to the resolved current $\overline{\boldsymbol{J}}=\nabla \times \overline{\boldsymbol{B}}$ in the EMF $\mathcal{E} \propto \eta_{t} \overline{\boldsymbol{J}}$ is used much more frequently. Similar to the eddy-viscosity, this resistivity (sometimes also referred to as anomalous resistivity) also allows for energy transfer only from the resolved to the unresolved scales by costruction (assuming that the resistivity is positive definite).

Structural modeling The main idea behind structural modeling is quite different from functional modeling. Here, the intrinsic properties of the SGS terms are modeled based on the assumption that the appropriate (manifold) functions follow automatically. Several models have originally been developed in hydrodynamics and also extended to MHD such as the scale-similarity model and deconvolution based models.

The scale-similarity model (here only the hydrodynamic closure)

$$
\tau_{i j}^{\mathrm{u}} \propto \overline{\bar{\rho}}\left({\widetilde{\widetilde{u_{i}}} \widetilde{u_{j}}}_{-}-{\widetilde{u_{i}}}_{\widetilde{u_{j}}}^{\widetilde{C}}\right)
$$

uses a second filter (also called test filter) with a characteristic width $\underbrace{}_{\Delta}$ larger than the initial filter $\Delta$. Bardina et al. (1980) observed that the scale-by-scale energy transfer is self-similar and deduced (Liu et al.|1994) that this idea also translates to the overall structure, i.e. $\tau(\Delta, \Delta) \sim \tau \hat{=} \tau\left(\Delta, \Delta_{x}\right)$. More precisely, the dynamics across the test filter scale $\Delta$ down to the filter scale $\Delta$ resemble the dynamics across the the filter scale $\Delta$ down to the (in this context) unknown dynamics at and below the grid-scale $\Delta_{x}$. Given the form of the closure, the turbulent energy flux has no definite sign any more and energy transfer is also possible from the unresolved to the resolved scales. This concept can straightforwardly extended to the MHD case in order to close the SGS Maxwell stress and the EMF as listed in table 1.1 .

Deconvolution based models try to reconstruct the information lost in the initial filtering, which is a convolution. Again, different approaches exist within this family, e.g. deconvolution based on Taylor expansion of the transfer function. Yeo (1987) introduced this nonlinear closure in hydrodynamics

$$
\tau_{i j}^{\mathrm{u}} \propto \frac{1}{12} \Delta^{2} \widetilde{\rho}_{i, k} \widetilde{u}_{j, k}
$$

which is related to the gradient-diffusion model (Leonard 1975, Clark et al. 1979) and here extended to compressible MHD, see chapter 2 and 3 for the derivation and table 1.1 for the result. The indices $\square_{i, j}$ indicate the $j$-th partial derivative of $i$-th component and summation of repeated indices applies. The major advantage of this kind of model is that its derivation does not rely on a particular feature, e.g. dissipation or self-similarity, of turbulence. Again, the form of the closure allows for a turbulent energy flux both forward from resolved to unresolved scales and inverse from unresolved to resolved ones. Another notable example in the family of deconvolution models is the approximate deconvolution approach by Stolz and Adams (1999), in which the deconvolution operation is directly applied to the primitive (filtered) quantities.

Finally, there exists a whole zoo of models which are constructed from the different approaches and with different regularization procedures to control unwanted behavior of a model. The most famous examples are mixed models and dynamic models. 
Mixed models (Zang et al. 1993) are typically constructed from a structural model that is combined with a purely dissipative model, e.g. a scale-similarity model complemented with an eddy-viscosity term. The motivation for this kind of regularization is that structural models allow for a backscatter of energy from the unresolved scales, which (in certain cases) results in unstable simulations (Vreman et al. 1997). This backscatter is then effectively suppressed by the additional dissipative term of the model.

Dynamic models (Germano et al. 1991, Lilly 1992) resort to a dynamic calculation of the model coefficient, which has not been mentioned yet. Basically all all models rely on an additional (ideally dimensionless) model coefficient. There has been a lot of work to determine coefficient values both theoretically and experimentally (or numerically). However, the coefficient value is virtually never universal. It has to be tuned so that the model is applicable in different situations. The dynamic procedure tries to circumvent this general deficiency. A second filter is applied to the governing equations and the resulting two new terms (in each equation) are statistically related to each other. This relation then allows for the determination of the coefficient, which is automatically adapted to the current state of the flow. This procedure has been proven to be quite successful in flows with boundary conditions, e.g. wall-bounded flows, where homogeneity is not provided any more.

\subsubsection{Explicit discrete filters}

In the case of explicit large eddy simulations, all SGS models are calculated based on resolved (filtered) quantities - even if a second additional filter is used. Usually, the computational grid is thought to be the implicit filter (even for explicit LES) and quantities at the grid-scale are considered to be resolved. However, this assumption strongly relies on the numerical method used. While this might be a good approximation within spectral schemes due to their high accuracy, it is a questionable approach in very dissipative schemes such as shock-capturing finite volume schemes (Garnier et al. 1999). In addition to this, the effective width and nature of the grid filter is unknown, i.e. the integrated behavior of discretization and numerical solvers does not naturally correspond, for example, to a box filter. For this reason, explicit filters can be used with filter widths larger than the grid-spacing so that their behavior dominates over the grid filter.

In general, a one dimensional, time independent filter of a function $\phi$ is defined by

$$
\bar{\phi}(x)=\int_{-\infty}^{+\infty} G(x-y) \phi(y) \mathrm{d} y
$$

with convolution kernel $G$. Similarly, a discrete filter on a uniform mesh with grid-spacing $\Delta_{x}$ is defined by

$$
\bar{\phi}_{i}=G \phi_{i}=\sum_{n=-M}^{N} a_{n} \phi_{i+n}
$$

with filter weights $a_{n}$ that are supported by a $M+N+1$ point stencil. Without loss of generality, the discussion is restricted to symmetric filters (i.e. $M=N$ and $a_{-n}=a_{n}$ ) as astrophysical flows are typically unbounded. For a discussion on asymmetric filters in complex geometries, see e.g. Vasilyev et al.(1998). 
Moreover, in order to construct a physically meaningful filter, preservation of constants and positiveness of the filter is required. The former leads to the following expression

$$
1=\sum_{n=-N}^{N} a_{n}=a_{0}+2 \sum_{n=1}^{N} a_{n} .
$$

Vreman et al. (1994) showed that for a positive filter

$$
a_{n}>0
$$

is necessary and sufficient to ensure the realizability of the SGS stress tensor and thus non-negative SGS energies. Both properties are fulfilled by the box and Gaussian filter, but not by the sharp cutoff filter.

Those three filters are the most commonly used filters in LES (Sagaut 2006). Their analytical kernels and transfer functions for a filter width $\Delta$ are in case of

- the box or top-hat filter

$$
G(x-y)=\left\{\begin{array}{ll}
\frac{1}{\Delta}, & \text { if }|x-y| \leq \frac{\Delta}{2} \\
0, & \text { otherwise }
\end{array} \quad \text { and } \quad \widehat{G}(k)=\frac{\sin k \Delta / 2}{k \Delta / 2},\right.
$$

- the Gaussian filter

$$
G(x-y)=\left(\frac{\gamma}{\pi \Delta^{2}}\right)^{1 / 2} \exp \left(\frac{-\gamma|x-y|^{2}}{\Delta^{2}}\right) \quad \text { and } \quad \widehat{G}(k)=\exp \left(\frac{-\Delta^{2} k^{2}}{4 \gamma}\right),
$$

- the spectral or sharp cutoff filter

$$
G(x-y)=\frac{\sin (\pi(x-y) / \Delta)}{\pi(x-y) / \Delta} \quad \text { and } \quad \widehat{G}(k)=\left\{\begin{array}{ll}
1, & \text { if }|k| \leq \frac{\pi}{\Delta} \\
0, & \text { otherwise }
\end{array} .\right.
$$

While the spectral filter is often applied in spectral schemes (for incompressible hydrodynamics) or theoretical analyses, it is less useful in the compressible regime. In addition to the already mentioned potential violation of realizability, it also allows for negative densities. Consequently, box and Gaussian filters are typically used in finite volume simulations of compressible (M)HD.

However, these filters in their analytical form cannot be readily applied in the simulation and need to be discretized first. One possibility is the approximation by polynomial truncation. Following Sagaut (2006), the discrete filter is constructed by matching the discrete filtering operator with the associated continuous differential operator up to arbitrary order. For this reason, both representations are approximated by a Taylor expansion. The expansion of the continuous operator 1.29 results in the following differential form

$$
\bar{\phi}(x)=\phi(x)+\sum_{l=1}^{\infty} \frac{(-1)^{l} \alpha^{(l)} \bar{\Delta}^{l}}{l !} \frac{\partial^{l} \phi(x)}{\partial x^{l}} \quad \text { with } \quad \alpha^{(l)}=\frac{1}{\bar{\Delta}^{l}} \int_{-\infty}^{+\infty} z^{l} G(z) \mathrm{d} z .
$$


Table 1.2: The first four non-zero moments of the box and Gaussian filter with filter width $\bar{\Delta}$

\begin{tabular}{lcccc}
\hline$\alpha^{(l)}$ & $l=0$ & $l=2$ & $l=4$ & $l=6$ \\
\hline box & 1 & $1 / 12$ & $1 / 80$ & $1 / 448$ \\
Gaussian & 1 & $1 / 12$ & $1 / 48$ & $1 / 576$ \\
\hline
\end{tabular}

$\alpha^{(l)}$ is the moment of order $l$ of the convolution kernel and $\bar{\Delta}$ the characteristic filter width. The first four non-zero moments of the box and Gaussian filter are given in table 1.2 .

Substituting $\phi_{i+n}$ in the discrete filter $(1.30)$ by the the Taylor expansion of $\phi$ around point $i$ leads to the following expression:

$$
\bar{\phi}_{i}=\left(1+\sum_{l=1}^{\infty} \frac{a_{l}^{*} \Delta_{x}^{l}}{l !} \frac{\partial^{l}}{\partial x^{l}}\right) \phi_{i} \quad \text { with } \quad a_{l}^{*}=\sum_{n=-N}^{N} a_{n} n^{l} .
$$

The discrete filter can now be constructed by matching the coefficients of $(1.36)$ and (1.37) up to desirable order $l$ resulting in an unclosed system of equations

$$
\epsilon^{2 l^{\prime}} \alpha^{\left(2 l^{\prime}\right)}=2 \sum_{n=1}^{N} a_{n} n^{2 l^{\prime}} \quad \text { for each } \quad l^{\prime}=1,2, \ldots, l / 2 \text {. }
$$

Odd moments do not enter the system for symmetric filters. The system is under-determined, in particular, by the ratio of filter width to grid spacing $\epsilon=\bar{\Delta} / \Delta_{x}$.

Together with the preservation of constants property (1.31), the resulting system can be solved for the coefficients by symbolic computation, e.g. as shown in listing A.1 in the appendix. The coefficients for the discrete box filter of 2nd, 4th, and 6th order, which are later used in chapter 5, are

$$
\begin{array}{ll}
l=2: & a_{0}=-\frac{1}{12} \epsilon^{2}+1, a_{1}=\frac{1}{24} \epsilon^{2}, \\
l=4: & a_{0}=\frac{1}{320} \epsilon^{4}-\frac{5}{48} \epsilon^{2}+1, a_{1}=-\frac{1}{480} \epsilon^{4}+\frac{1}{18} \epsilon^{2}, a_{2}=\frac{1}{1920} \epsilon^{4}-\frac{1}{288} \epsilon^{2}, \\
l=6: & a_{0}=-\frac{1}{16128} \epsilon^{6}+\frac{7}{1440} \epsilon^{4}-\frac{49}{432} \epsilon^{2}+1, a_{1}=\frac{1}{21504} \epsilon^{6}-\frac{13}{3840} \epsilon^{4}+\frac{1}{16} \epsilon^{2}, \\
& a_{2}=-\frac{1}{53760} \epsilon^{6}+\frac{1}{960} \epsilon^{4}-\frac{1}{160} \epsilon^{2}, a_{3}=\frac{1}{322560} \epsilon^{6}-\frac{1}{11520} \epsilon^{4}+\frac{1}{2160} \epsilon^{2} .
\end{array}
$$

Choosing some value for $\epsilon$ readily closes the system and uniquely determines all coefficients. However, the choice is not entirely free. Some values of $\epsilon$ result in coefficients violating the positiveness condition (1.32). Moreover, the filter width width should not be chosen larger than the available stencil or smaller than $\Delta_{x}$. One alternative approach to choose some $\epsilon$ is to make use of this degree of freedom to add additional desirable properties to the filter. Several approaches exists in literature usually referred to as additional filter constraints. 
Case Ia: $\widehat{G}\left(\pi / \Delta_{x}\right)=0$, i.e. the transfer function of the filter is null at the grid cutoff, has been suggested by Vasilyev et al. (1998). The discrete filter kernel (1.30) can be expressed by a sum of Dirac $\delta$-functions. Thus, the transfer function is given by

$$
\widehat{G}(k)=\sum_{n=-M}^{N} a_{n} e^{j k n \Delta_{x}}, \quad \text { with } \quad j^{2}=-1 .
$$

For a symmetric filter the complex transfer function reduces to the following real transfer function

$$
\widehat{G}(k)=a_{0}+2 \sum_{n=1}^{N} a_{n} \cos k n \Delta_{x},
$$

This leads to a modification of amplitudes only and no phase shifts are introduced. Eventually, the additional equation for the coefficient values is

$$
\widehat{G}\left(\pi / \Delta_{x}\right)=a_{0}+2 \sum_{n=1}^{N}(-1)^{n} a_{n}=0 .
$$

The coefficient values of the box filter of order 2, 4 and 6 are listed in table A.1 where combinations resulting in a non-positive filter have been neglected.

Case Ib: $\widehat{G}^{\mathrm{dis}}\left(\pi / \Delta_{x}\right)=\widehat{G}^{\text {ana }}\left(\pi / \Delta_{x}\right)$, i.e. matching transfer function values of the discrete and the analytic filter at grid cutoff as suggested by Schmidt (2004). For the box filter, the additional constraint is

$$
\frac{2 \sin (\epsilon \pi / 2)}{\epsilon \pi}=a_{0}+2 \sum_{n=1}^{N}(-1)^{n} a_{n} .
$$

Again, several solutions are possible as shown in table A.1

Case Ic: $\widehat{G}(\pi / \bar{\Delta})=1 / 2$, i.e. the "filter width is taken to be proportional to the inverse wavenumber where the filter transfer function falls to 0.5" Lund (1997). This has also been used by Vasilyev et al. (1998) to constrain their filters by enforcing

$$
\frac{1}{2}=a_{0}+2 \sum_{n=1}^{N} a_{n} \cos \left(\frac{\pi n}{\epsilon}\right) .
$$

However, for the box filter, no solutions exists for 3-, 5- and 7-point stencils.

In contrast to matching coefficients up to arbitrary order for one additional constraint at a specific scale, another approach yielding so called optimal filters has been proposed by Sagaut and Grohens (1999), Vasilyev et al. (1998). Here, the overall residual between analytic and discrete filter given by

$$
\int_{0}^{\pi / \square}\left(\widehat{G}(k)-\widehat{G}_{\mathrm{d}}(k)\right)^{2} \mathrm{~d} k
$$

is minimized. Again, different realizations are possible depending on the range of integration. 
Case IIa: Integration over $\left[0, \pi / \Delta_{x}\right]$ has been suggested by Vasilyev et al. (1998). For a discrete box filter the functional to minimize is

$$
\int_{0}^{\pi}\left(\frac{\sin \left(k^{\prime} \epsilon / 2\right)}{k^{\prime} \epsilon / 2}-2 \sum_{n=1}^{N}\left(a_{n}\left(\cos \left(k^{\prime} n\right)-1\right)\right)-1\right)^{2} \mathrm{~d} k^{\prime},
$$

where the constraint of preservation of constants (1.31) is already included. A sample code for a 3-point stencil is given in listing A.2. In general, there exists multiple local minima for a given number of supporting nodes. All solutions are shown in table A.1 and the solution with the lowest residual is marked by a ' $*$ '.

Case IIb: Integration over $[0, \pi / \widehat{\Delta}]$ is used by Sagaut and Grohens $(1999)$, i.e. they integrate only wavenumbers below the filter width. Here, the functional is

$$
\int_{0}^{\pi}\left(\frac{\sin \left(k^{\prime} / 2\right)}{k^{\prime} / 2}-2 \sum_{n=1}^{N}\left(a_{n}\left(\cos \left(k^{\prime} n / \epsilon\right)-1\right)\right)-1\right)^{2} \mathrm{~d} k^{\prime} .
$$

Again, the coefficients are given in table A.1.

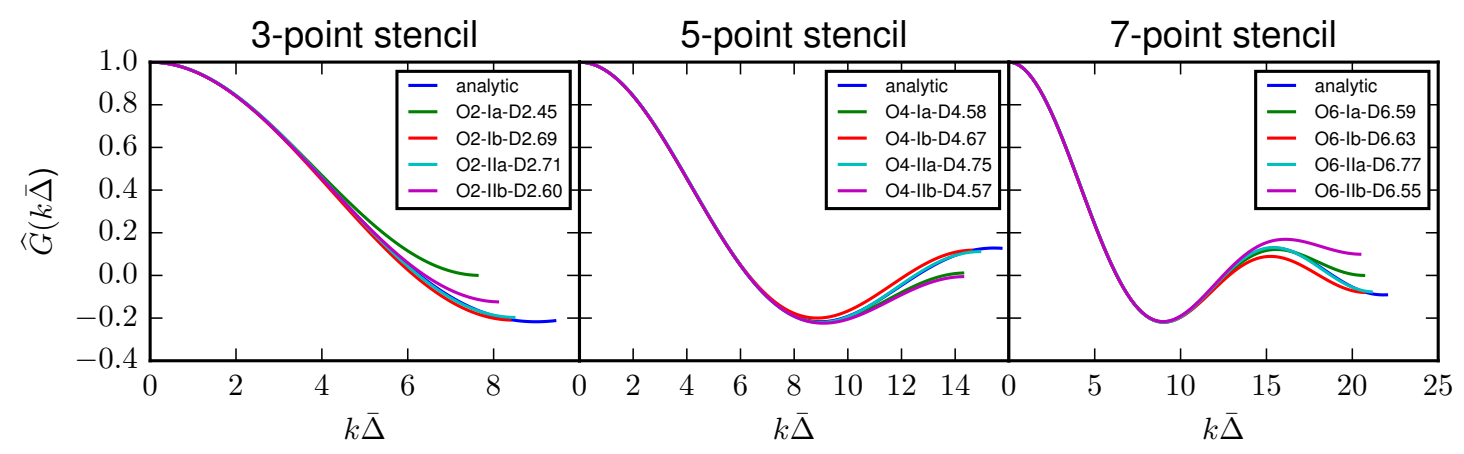

Figure 1.2: Comparison of discrete filter approximations to the analytical box filter for different constraints and stencil sizes.

The resulting filters for all discretization approaches using 3-, 5- and 7-point stencils are illustrated in figure 1.2. In general, the approaches that use the grid cutoff as additional constraint, i.e. case Ib in the polynomial truncation approach and case IIa for the optimal filter approach, provide better approximations to the continuous filter than using a filter width based constraint. Moreover, the differences between Ib and IIa are almost negligible for the two smallest stencils. First for larger stencils (and larger filter widths) the superiority of the optimal filter is distinguishable as shown in figure 1.2 .

The final step towards an implementable discrete filter for LES is the extension to multiple dimensions. Generally, two straightforward approaches based on the construction by one-dimensional filters are possible (Sagaut and Grohens 1999): either simultaneous application in all dimensions or sequential application.

In the simultaneous case, the three-dimensional filter is constructed as

$$
\bar{\phi}_{i, j, k}=\sum_{l=-N}^{N} a_{l}\left(\phi_{i+l, j, k}+\phi_{i, j+l, k}+\phi_{i, j, k+l}\right)
$$


for a (symmetric) $2 N+1$-point one-dimensional filter. Thus, the resulting filter has $6 N+1$ supporting points. The sequential application of this filter corresponds to the following operation

$$
\bar{\phi}_{i, j, k}=\sum_{l=-N}^{N} \sum_{m=-N}^{N} \sum_{n=-N}^{N} a_{l} a_{m} a_{n} \phi_{i+l, j+m, k+n} .
$$

Here, the number of supporting points for the multi-dimensional stencil is greatly increased to $(2 N+1)^{3}$.

Sagaut and Grohens (1999) evaluated the differences between these two approaches in combination with polynomial truncation based box filters and optimized box filters by applying them to a von Karmann spectrum. They find that the error between the filtered discrete spectrum and the analytic spectrum is greatly reduced when a) an optimized filter is used and b) the multi-dimensional filter is constructed based on sequential application. For this reason, these kind of filters are later used in the LES presented in chapter5.

\subsubsection{Verification and validation}

One important aspect of large eddy simulations is the verification and validation of the framework, no matter if it is the implicit scheme or an explicit subgrid-scale model. On the one hand, verification refers to testing whether assumptions in the derivation are met or, for example, how well an SGS model approximates the analytical solution in a given situation. Given that the SGS models take no active part in these tests, they are considered as an a priori analysis. On the other hand, validation refers to testing how the entire framework performs in practice and is vernacularly identified with the term a posteriori. Here, the integrated nature of e.g. numerical scheme and explicit SGS model is tested. Both steps are necessary for successful LES.

In this thesis, both kind of tests are performed. Chapters 2 and 4 present $a$ priori tests of several SGS models, and chapter 5 demonstrates a posteriori validation of selected models for decaying, supersonic MHD turbulence.

All tests have a comparison to some reference data or simulation in common. This comparison is straightforward in a priori tests, where reference data, e.g. from experiments or from high resolution (direct) numerical simulations, can easily be filtered. This filtering results in no reduction of resolution, but in a separation of scales while simultaneously retaining information from the large and small scales. Having quantities on all scales available allows for both the exact (analytical) calculation of subgrid-scale quantities and predicted values by models based on the large-scale quantities only. A comparison between exact and predicted value, for example, by means of a correlation coefficient, can then be used to quantify and rate the performance of different SGS models.

The a posteriori evaluation works similar. However, there are some subtle differences given that actual simulations with and without SGS models at different resolutions are usually compared. The most common technique, see e.g. Müller and Carati (2002), is to use a high-resolution reference simulation without an SGS model and apply a coarsegraining procedure to the data. Coarse-graining corresponds to an effective reduction in resolution, for example, when using volume averages over $2^{3}$ cells, i.e. a non-moving box filter with $\Delta=2 \Delta_{x}$, in order to reduce the resolution by a factor of 2 in each direction. 
Data at lower resolution can then directly be compared to results from explicit LES that were run at a lower resolution from the beginning. This procedure has the advantages that quantities, which have a strong resolution dependence such as derivatives, are calculated on equal grounds.

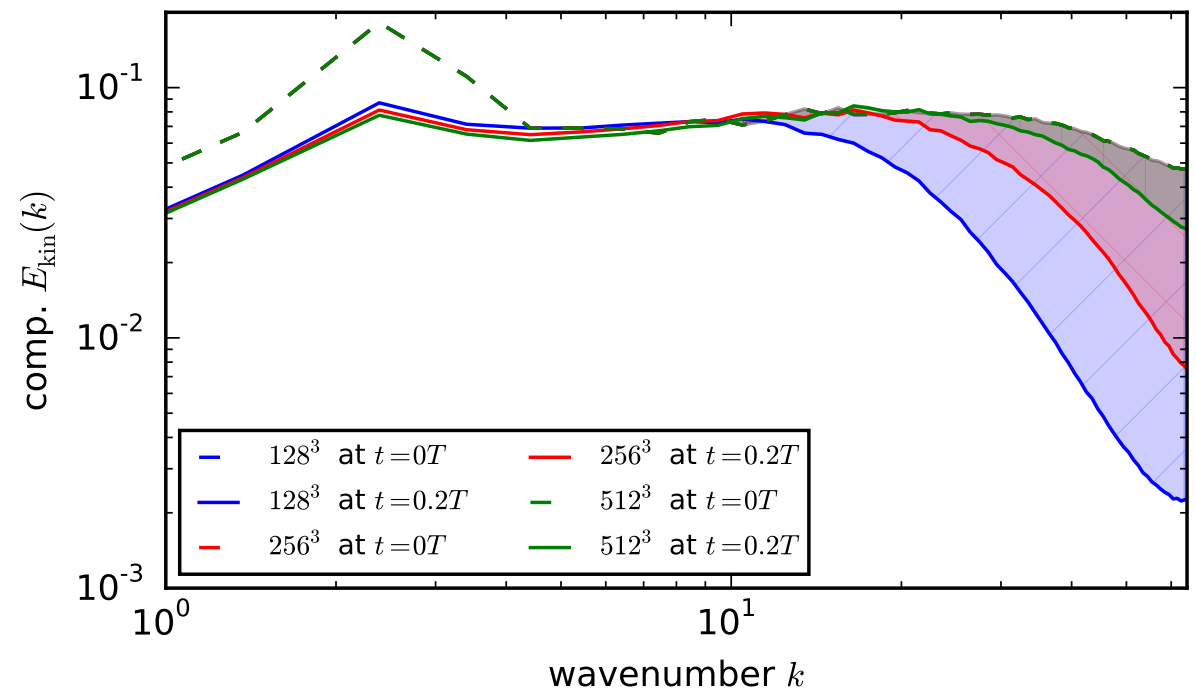

Figure 1.3: Initial compensated kinetic energy spectra of a turbulence-in-a-box simulation for different resolutions. All spectra are analyzed at a resolution of $128^{3}$, i.e. coarsegraining has been applied to the higher-resolution data. The spectra at $t=0 T$ are identical and derived from a reference simulation at $1024^{3}$. The excess energy on the smallest scales due to increased numerical dissipation at lower resolution after $0.2 T$ of free decay is indicated by the shaded ares.

Finally, going one step back, coarse-graining of high-resolution data is also used to generate initial conditions for lower resolution simulations as e.g. in chapter 5 or in Kritsuk et al. (2011). Here, special care needs to be taken as the procedure (especially in shock-capturing finite volume schemes) leaves excess energy on the small scales. Figure 1.3 illustrates this excess energy due to increased numerical dissipation at lower resolutions. If the initial ( $t=0)$ energy spectra would be used as initial conditions for lower resolution LES with explicit SGS model, results would be difficult to interpret because the interaction between model and nonphysical (for a given resolution) energy can hardly be quantified. For this reason, the initial conditions are first evolved without model for transient phase of $t=0.2 T$ turnover times where the excess energy is allowed to relax to a stationary state. Eventually, this enables a direct comparison of different SGS models to a reference run all with (almost) identical initial conditions. The alternative approach used e.g. by Vreman et al. (1992) is to generate an artificial energy spectrum that is fully resolved at the lowest resolution as initial condition in all resolutions. 



\section{Paper I: Nonlinear closures for scale separation in supersonic magnetohydrodynamic turbulence}

The paper originally appeared as Philipp Grete, Dimitar G Vlaykov, Wolfram Schmidt, Dominik R G Schleicher, and Christoph Federrath. Nonlinear closures for scale separation in supersonic magnetohydrodynamic turbulence. New Journal of Physics, 17(2): 023070, 2015. doi : 10.1088/1367-2630/17/2/023070.

The research was jointly designed and planned by all authors. PG originally proposed the new closures. The text and the analysis framework was jointly written and developed by DGV and PG in constant exchange with the coauthors. PG adapted the original implemention of the forcing mechanism by Schmidt et al. (2009) to the latest ENzo version and took care of the upstream integration. CF contributed the FLASH simulations and the Enzo simulations were run by PG. 


IOP Publishing $\quad$ New J. Phys. 17(2015) 023070 doi:10.1088/1367-2630/17/2/023070

\section{New Journal of Physics}

The open access journal at the forefront of physics

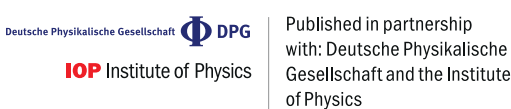

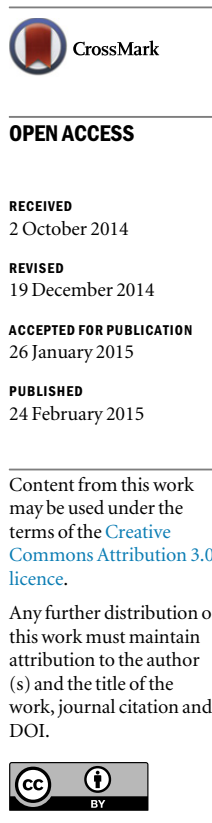

PAPER

\section{Nonlinear closures for scale separation in supersonic} magnetohydrodynamic turbulence

\author{
Philipp Grete ${ }^{1,2}$, Dimitar G Vlaykov ${ }^{2}$, Wolfram Schmidt ${ }^{2}$, Dominik R G Schleicher ${ }^{2}$ and \\ Christoph Federrath ${ }^{3}$ \\ 1 Max-Planck-Institut für Sonnensystemforschung, Justus-von-Liebig-Weg 3, D-37077 Göttingen, Germany \\ 2 Institut für Astrophysik, Universität Göttingen, Friedrich-Hund-Platz 1, D-37077 Göttingen, Germany \\ 3 Research School of Astronomy \& Astrophysics, The Australian National University, Canberra, ACT 2611, Australia \\ E-mail: grete@mps.mpg.de \\ Keywords: magnetohydrodynamics, turbulence, subgrid-scale closure, scale separation \\ Abstract
}

Turbulence in compressible plasma plays a key role in many areas of astrophysics and engineering. The extreme plasma parameters in these environments, e.g. high Reynolds numbers, supersonic and super-Alfvenic flows, however, make direct numerical simulations computationally intractable even for the simplest treatment-magnetohydrodynamics (MHD). To overcome this problem one can use subgrid-scale (SGS) closures-models for the influence of unresolved, subgrid-scales on the resolved ones. In this work we propose and validate a set of constant coefficient closures for the resolved, compressible, ideal MHD equations. The SGS energies are modeled by Smagorinsky-like equilibrium closures. The turbulent stresses and the electromotive force (EMF) are described by expressions that are nonlinear in terms of large scale velocity and magnetic field gradients. To verify the closures we conduct a priori tests over 137 simulation snapshots from two different codes with varying ratios of thermal to magnetic pressure $\left(\beta_{\mathrm{p}}=0.25,1,2.5,5,25\right)$ and sonic Mach numbers $\left(M_{\mathrm{s}}=2,2.5,4\right)$.

Furthermore, we make a comparison to traditional, phenomenological eddy-viscosity and $\alpha-\beta-\gamma$ closures. We find only mediocre performance of the kinetic eddy-viscosity and $\alpha-\beta-\gamma$ closures, and that the magnetic eddy-viscosity closure is poorly correlated with the simulation data. Moreover, three of five coefficients of the traditional closures exhibit a significant spread in values. In contrast, our new closures demonstrate consistently high correlations and constant coefficient values over time and over the wide range of parameters tested. Important aspects in compressible MHD turbulence such as the bi-directional energy cascade, turbulent magnetic pressure and proper alignment of the EMF are well described by our new closures.

\section{Introduction}

Turbulence is ubiquitous in astrophysical plasmas, ranging from coronal mass ejections and stellar winds [1], through star formation in molecular clouds [2], to the gas in the interstellar [3] and intracluster medium. While experimental setups [4] become increasingly more realistic, they are still far away from the regime acting in such extreme conditions. For numerical simulations it is computationally too expensive (if even possible) to capture the entire range of physical processes from plasma kinetics to the integral scales of turbulence. In an astrophysical context, one has to further contend with the additional complications brought about by high compressibility and the accompanying supersonic and super-Alfvenic motion.

Possible ways to circumvent the infeasibility of direct numerical simulations are the use of calculations based on mean-field theories or large-eddy simulations [5]. These simulations only resolve the energy containing large scale dynamics and require a subgrid-scale (SGS) model to account for unresolved effects. While a lot of research has been successfully carried out in the realm of hydrodynamics [6], compressible magnetohydrodynamic SGS closures are essentially unexplored. Previous research is mainly based on the concept of turbulent dissipation in 
incompressible flows [7-10]. They expand the idea of a turbulent eddy-viscosity to an additional eddy-resistivity in the induction equation and propose different phenomenological models. Even though these models are then evaluated a posteriori, a general verification and justification a priori has so far only been considered for a single incompressible dataset [11]. Thus, our objective is to establish the validity of closures for the filtered, compressible magnetohydrodynamics (MHD) equations by coarse-graining multiple datasets from highresolution simulations of statistically homogeneous, forced MHD turbulence.

In general, the effect of finite resolution in numerical simulations can be mimicked by applying a low-pass filter to the standard, ideal MHD equations. This is achieved by convolving the equations with a suitable filter kernel $G$. See e.g. Garnier et al [6] for details on the properties of low-pass filtering and the conditions that $G$ needs to satisfy. For a homogeneous, isotropic, stationary kernel, under periodic boundary conditions [12] the equations take the following form

$$
\begin{aligned}
& \frac{\partial \bar{\rho}}{\partial t}+\nabla \cdot(\bar{\rho} \tilde{\boldsymbol{u}})=0, \\
& \frac{\partial \bar{\rho} \tilde{\boldsymbol{u}}}{\partial t}+\nabla \cdot(\bar{\rho} \tilde{\boldsymbol{u}} \otimes \tilde{\boldsymbol{u}}-\overline{\boldsymbol{B}} \otimes \overline{\boldsymbol{B}})+\nabla\left(\bar{P}+\frac{\bar{B}^{2}}{2}\right)=-\nabla \cdot \tau, \\
& \frac{\partial \overline{\boldsymbol{B}}}{\partial t}-\nabla \times(\tilde{\boldsymbol{u}} \times \overline{\boldsymbol{B}})=\nabla \times \mathcal{E} .
\end{aligned}
$$

The units of the magnetic field $\overline{\boldsymbol{B}}$ incorporate $1 / \sqrt{4 \pi}$. An overbar $\square$ denotes $^{4}$ filtered and a tilde $\widetilde{\square}$ massweighted filtered quantities [12]. For instance, the filtered density field is given by $\bar{\rho}=G * \rho$, while the massweighed filtered velocity field is $\tilde{\boldsymbol{u}}=\overline{\boldsymbol{u}} \rho / \bar{\rho}$. In this formalism, all filtered primary quantities, density $\bar{\rho}$, velocity $\tilde{\boldsymbol{u}}$, magnetic field $\overline{\boldsymbol{B}}$, and thermal pressure $\bar{P}$ are presumed to be known and directly accessible. Due to the introduction of mass-weighted filtering the only remaining terms that require closure are the SGS stress $\tau$ and the electromotive force $(\mathrm{EMF}), \mathcal{E}$. They are analytically expressed $[10]$ as

$$
\begin{gathered}
\mathcal{E}=\overline{\boldsymbol{u} \times \boldsymbol{B}}-\tilde{\boldsymbol{u}} \times \overline{\boldsymbol{B}} \text { and } \\
\tau_{i j}=\tau_{i j}^{\mathrm{u}}-\tau_{i j}^{\mathrm{b}}+\left(\overline{B^{2}}-\bar{B}^{2}\right) \frac{\delta_{i j}}{2}, \quad \text { with } \\
\tau_{i j}^{\mathrm{u}} \equiv \bar{\rho}\left(\widetilde{u_{i} u_{j}}-\tilde{u}_{i} \tilde{u}_{j}\right) \quad \text { and } \tau_{i j}^{\mathrm{b}} \equiv\left(\overline{B_{i} B_{j}}-\bar{B}_{i} \bar{B}_{j}\right) .
\end{gathered}
$$

The SGS stress tensor can be decomposed into the well-known turbulent Reynolds stress $\tau^{\mathrm{u}}$, a turbulent Maxwell stress $\tau^{\mathrm{b}}$ and a magnetic pressure term.

Furthermore, the definitions of the SGS energies are obtained from applying the filter to the total filtered energy density $\bar{E}$, which can be decomposed into the contribution due to resolved fields only and a remainder, designated as SGS energy

$$
\bar{E}=\underbrace{\frac{1}{2} \bar{\rho} \widetilde{u}^{2}+\frac{1}{2} \bar{B}^{2}}_{\text {(resolved) }}+\underbrace{\frac{1}{2} \bar{\rho}\left(\widetilde{u^{2}}-\tilde{u}^{2}\right)+\frac{1}{2}\left(\overline{B^{2}}-\bar{B}^{2}\right)}_{=E_{\mathrm{sg}}^{\mathrm{u}}+E_{\mathrm{sg}}^{\mathrm{b}}=E_{\mathrm{sgs}}} .
$$

It is important to point out that in general the filtering operator is not a Reynolds operator, in particular $\bar{\square} \neq$. It follows that SGS terms, like $E_{\mathrm{SGS}}$, carry information not only about the interactions between unresolved fields but also about cross-scale interactions between unresolved and resolved fields. In addition to this, the turbulent magnetic pressure is identical to the magnetic SGS energy $E_{\mathrm{sgs}}^{\mathrm{b}}$ and both kinetic and magnetic SGS energies are directly given by $2 E_{\mathrm{sgs}}^{\mathrm{u}}=\operatorname{Tr}\left(\tau^{\mathrm{u}}\right)$ and $2 E_{\mathrm{sgs}}^{\mathrm{b}}=\operatorname{Tr}\left(\tau^{\mathrm{b}}\right)$, i.e. they constitute the isotropic parts of the respective SGS tensors. Following the general tensor decomposition, the deviatoric, traceless parts are then given by $\tau_{i j}^{\square *}=\tau_{i j}^{\square}-\frac{1}{3} \delta_{i j} \tau_{k k}^{\square}$.

\section{Traditional closures}

In hydrodynamics, the traceless part of the SGS stress tensor is commonly closed by means of the eddy-viscosity hypothesis $\hat{\tau}_{i j}^{\mathrm{u} \star}=-2 \nu^{\mathrm{u}} \bar{\rho} \widetilde{S}_{i j}^{\star}$ in analogy to the molecular viscosity term in the momentum equation, where $\widetilde{S}_{i j} \equiv \frac{1}{2}\left(\tilde{u}_{i, j}+\tilde{u}_{j, i}\right)$ is the filtered kinetic rate-of-strain tensor. This introduces a turbulent kinetic eddyviscosity $\nu^{\mathrm{u}}=C_{\nu}^{\mathrm{u}} \Delta\left(E_{\mathrm{sgs}}^{\mathrm{u}} / \bar{\rho}\right)^{1 / 2}$ which is proportional to a characteristic velocity, commonly given by the kinetic SGS energy, and a characteristic length scale $\Delta$. This closure has already been applied directly to MHD $[7,13]$ by

${ }^{4}$ Throughout the paper the symbol $\square$ is used as a generic placeholder for variables. $\widehat{\square}$ designates closure expressions. Furthermore, we employ Einstein summation convention and $\square_{i, k}$ is identified with the $k$ th partial derivative of the $i$ th component of $\square$. 


\section{Paper I: Nonlinear closures for scale separation in supersonic MHD turbulence}

neglecting the magnetic contribution $\tau^{\mathrm{b}}$ in the momentum equation. A turbulent magnetic viscosity $\nu^{\mathrm{b}}=C_{\nu}^{\mathrm{b}} \Delta\left(E_{\mathrm{sgs}}^{\mathrm{b}}\right)^{1 / 2}$ was used in [10] with the closure

$$
\hat{\tau}_{i j}^{\mathrm{b} *}=-2 \nu^{\mathrm{b}} \overline{\mathcal{M}_{i j}},
$$

where $\overline{\mathcal{M}}_{i j} \equiv \frac{1}{2}\left(\bar{B}_{i, j}+\bar{B}_{j, i}\right)$ is the filtered magnetic rate-of-strain tensor.

The SGS energies can either be determined by individual evolution equations, where several terms again require closure, or by an instantaneous closure. Smagorinsky [14] introduced such an instantaneous closure in pure incompressible hydrodynamics $(\boldsymbol{B}=0)$ by assuming the SGS energy flux to be in equilibrium with the rate of dissipation

$$
\widehat{E}_{\mathrm{sgs}}^{\mathrm{u}}=C_{\mathrm{E}}^{\mathrm{u}} \Delta^{2} \rho\left|\widetilde{S}^{*}\right|^{2}
$$

Here, $\left|\widetilde{S}^{\star}\right| \equiv \sqrt{2 \widetilde{S_{i j}^{*} \widetilde{S_{i j}^{*}}}}$ denotes the rate-of-strain magnitude.

Finally, the EMF is commonly modeled (e.g. [7, 8, 10,13]) by variations of [15]

$$
\widehat{\mathcal{E}}=\alpha \overline{\boldsymbol{B}}-\beta \overline{\boldsymbol{J}}+\gamma \widetilde{\boldsymbol{\Omega}},
$$

with resolved current $\overline{\boldsymbol{J}}=\nabla \times \overline{\boldsymbol{B}}$ and vorticity $\widetilde{\boldsymbol{\Omega}}=\nabla \times \tilde{\boldsymbol{u}}$. The coefficients $\alpha, \beta$, and $\gamma$ are typically related to the $\alpha$-effect, turbulent resistivity and turbulent cross helicity, respectively. The commonly used closures for these coefficients

$$
\alpha=C_{\alpha}^{\mathcal{E}} t_{\text {turb }} H, \quad \beta=C_{\beta}^{\mathcal{E}} t_{\text {turb }} E_{\mathrm{sgs}} / \bar{\rho}, \quad \gamma=C_{\gamma}^{\mathcal{E}} t_{\text {turb }} W,
$$

are based on dimensional arguments, with turbulent cross helicity $W=\overline{\boldsymbol{u} \cdot \boldsymbol{B}}-\tilde{\boldsymbol{u}} \cdot \overline{\boldsymbol{B}}$, residual helicity $H \sim(\overline{\boldsymbol{J} \cdot \boldsymbol{B}}-\overline{\boldsymbol{J}} \cdot \overline{\boldsymbol{B}})-\bar{\rho}(\overline{\boldsymbol{\Omega} \cdot \boldsymbol{u}}-\widetilde{\boldsymbol{\Omega}} \cdot \tilde{\boldsymbol{u}})$, and timescale $t_{\mathrm{turb}}=\Delta\left(E_{\mathrm{sgs}} / \bar{\rho}\right)^{-1 / 2}$.

\section{Nonlinear closures}

In our new approach we adopt the compressible hydrodynamic nonlinear closure for the kinematic deviatoric stress tensor $\tau^{\mathrm{u} *}$ from [16], similar to the incompressible one from [17]. We propose the straightforward extension to MHD with

$$
\begin{aligned}
& \hat{\tau}_{i j}^{\mathrm{u} *}=2 C_{\mathrm{nl}}^{\mathrm{u}} E_{\mathrm{sgs}}^{\mathrm{u}}\left(\frac{\tilde{u}_{i, k} \tilde{u}_{j, k}}{\tilde{u}_{l, s} \tilde{u}_{l, s}}-\frac{1}{3} \delta_{i j}\right), \\
& \hat{\tau}_{i j}^{\mathrm{b} *}=2 C_{\mathrm{nl}}^{\mathrm{b}} E_{\mathrm{sgs}}^{\mathrm{b}}\left(\frac{\bar{B}_{i, k} \bar{B}_{j, k}}{\bar{B}_{l, s} \bar{B}_{l, s}}-\frac{1}{3} \delta_{i j}\right) .
\end{aligned}
$$

The tensorial structure, e.g. $\tilde{u}_{i, k} \tilde{u}_{j, k}$, can be obtained by a Taylor expansion discarding terms with 2 nd and higher order gradients of the resolved fields. The overall normalization with the SGS energies comes from the constraint that the SGS stresses vanish in laminar flows.

Applying the nonlinearity idea to the EMF generalizes the closure proposed by [11] to the compressible regime

$$
\widehat{\mathcal{E}}_{i}=\varepsilon_{i j k} C_{\mathrm{nl}}^{\mathcal{E}} \Delta^{2} \tilde{u}_{j, s} \bar{B}_{k, s} .
$$

The closure explicitly preserves the anti-symmetry between velocity and magnetic field in $\mathcal{E}$, which in turn helps in capturing their relative geometry.

Finally, to complete the set of nonlinear closure equations, we use the Smagorinsky expression for the turbulent kinetic energy (3) and propose an analogous extension to the magnetic part

$$
\widehat{E}_{\mathrm{sgs}}^{\mathrm{b}}=C_{\mathrm{E}}^{\mathrm{b}} \Delta^{2}|\overline{\mathcal{M}}|^{2} .
$$

Here, the turbulent magnetic energy is proportional to the magnetic rate-of-strain magnitude $|\overline{\mathcal{M}}| \equiv \sqrt{2 \overline{\mathcal{M}_{i j}} \overline{\mathcal{M}_{i j}}}$. There are two advantages of closing $E_{\text {sgs }}^{\mathrm{u}}$ and $E_{\text {sgs }}^{\mathrm{b}}$ separately and not jointly via the total SGS energy. First, there is no additional need to close the often neglected turbulent magnetic pressure, as it is given by $E_{\text {sgs. }}^{\mathrm{b}}$. Second, the individual energies provide closures to the isotropic parts of the turbulent stress tensors $\tau^{\mathrm{u}}$ and $\tau^{\mathrm{b}}$. 


\section{Validation method}

In order to evaluate the proposed closures, we perform an a priori comparison using simulation data obtained from two, grid-based MHD codes (ENzo[18] and FLASHv4[19]). This way the results are less likely to hinge on the particulars of the numerical implementation. In both cases we follow the evolution of a compressible, isothermal fluid in a cubic box with resolution of $512^{3}$ grid cells and periodic boundary conditions, starting from uniform initial conditions. In Enzo we use an ideal equation of state with adiabatic exponent $\kappa=1.001$ in order to approximate isothermal gas. ENzo is a finite-volume code, i.e. the evolution equations are evaluated in integral form by solving a Riemann problem for the mass, momentum and energy flux through cell walls. This allows for the conservation of MHD invariants (e.g. energy) to machine precision. We use a MUSCL-Hancock scheme [20] (a second-order accurate Godunov extension) with second-order Runge-Kutta time integration and Harten-Lax-van Leer (HLL) Riemann solver (a two-wave, three-state solver) to solve the ideal MHD equations. The FLASHv 4 code is similar to ENzo (second-order accurate in space and time), but uses the positive-definite HLL3R Riemann solver [20]. Another difference is that FLASHv4 uses a polytropic equation of state to keep the gas exactly isothermal. Moreover, explicit kinematic viscosity and magnetic resistivity terms are included in the momentum, energy, and induction equations. We set the kinematic and magnetic Reynolds numbers to $\mathrm{Re}=\mathrm{Rm}=3780$. Consequently, the magnetic Prandtl number $\mathrm{Pm}=\mathrm{Rm} / \mathrm{Re}$ is unity. For details on the numerical methods used in FLASHv4, including viscous and resistive dissipation, see [21] and [22]. Both codes employ divergence cleaning [23] to maintain $\nabla \cdot \boldsymbol{B}=0$. A state of homogeneous and isotropic turbulence is reached by supersonic stochastic driving in the momentum equation (given by an Ornstein-Uhlenbeck process) at small wave-numbers, similar to $[24,25]$. Thus, the forcing field is evolving in time and space. The associated large auto-correlation time-scale $T$ of the forcing translates to the eddy turnover time of the largest, energycontaining eddies. It is therefore the chosen unit of time in the following.

We explore a range of parameters. The initial strength of the magnetic field is set by the plasma $\beta_{\mathrm{p}}$-the ratio of thermal to magnetic pressure. The final sonic Mach number $M_{\mathrm{s}}$ is determined by the forcing amplitude. For the Enzo simulations we have initial $\beta_{\mathrm{p}}=0.25,2.5,25$, with $M_{\mathrm{s}} \approx 2.5$ after $t \approx 2 T$ turnover times. The FLASHv4 simulations reach $M_{\mathrm{s}} \approx 4,2$ for initial $\beta_{\mathrm{p}}=1,5$, keeping instead constant Alfvenic Mach number $M_{\mathrm{a}} \approx 3$. We discard all initial data affected by transients (before a simulation time of $t=2 T$ ) and analyze consequent snapshots taken approximately in intervals of $0.15 T$ and $0.1 T$ for the ENZO and FLASHv 4 datasets, respectively.

The analysis begins with the application of a low-pass Gaussian (test) filter to the equations of motion. In the context of the closures we investigate, filtered quantities (i.e. density, velocity, and magnetic field) are interpreted as resolved, while the remainders represent the unresolved small scales. We can then compute $\tau$ and $\mathcal{E}$ both directly from (1) and (2), and from their respective traditional and nonlinear closures.

The determination of the length-scale of the filter bears some consideration. It needs to fall within an intermediate range of length scales, away from the particular effects of both the large-scale forcing and the smallscale dissipation. The largest scale of the system is the full box size $L$ and corresponds to the wavenumber $n=1$, while the smallest scale is given by the Nyquist wavenumber $n_{\mathrm{Nyq}}=N / 2=256$ for the linear numerical resolution $N=512$ grid cells. The turbulence injection wavenumber is $n_{\text {inj }}=2$ (corresponding to half the box size $L / 2$ ) in both codes, which is why the energy spectra, as illustrated in figure 1, peak there. The figure shows the mean kinetic and total (kinetic plus magnetic) energy spectra as a function of wavenumber. Since the stochastic forcing is implemented only in the momentum equation both for ENzO and FLASHv4, the kinetic energy spectrum exhibits the most direct imprint of the forcing itself. Conversely, the total energy spectrum carries the overall effect of the small-scale dissipation through both kinetic and magnetic channels. Figure 1 demonstrates that our simulations produce approximate power-law scaling within a narrow range of wavenumbers, which is indicative of self-similar turbulent fluctuations [26]. This can be interpreted as inertial range dynamics, although the nature of the inertial range in compressible MHD turbulence is still not fully understood [27-30]. Furthermore, this range separates the forcing scale and the dissipation scales and is not affected by numerical diffusion in the absence of a bottleneck effect as demonstrated by [31]. The vertical dotted line in figure 1 indicates our chosen filter length scale, corresponding to $\Delta=16$ grid cells or wavenumber $n_{\text {filter }}$ $=N /(2 \Delta)=16$. This filter scale falls within the range of the self-similar power-law range for both the kinetic and total energy spectra. This is why we use this ideal scale for our filter in the following analysis.

Additionally, this provides the motivation to treat data from both simulations on equal footing, even though FLASHv4 has explicit viscosity and diffusivity while Enzo solves the ideal MHD equation, subject to numerical dissipation only.

In order to incorporate coordinate independence, a scalar field is chosen for comparison, the SGS energy flux $\Sigma$, i.e. the term responsible for the transfer of SGS energy between resolved and unresolved scales. Its components associated with the Reynolds and Maxwell stresses and the EMF are $\Sigma^{u}=\tau_{i j}^{u} \widetilde{S}_{i j}, \Sigma^{b}=\tau_{i j}^{b} \widetilde{S}_{i j}$ and 


\section{Paper I: Nonlinear closures for scale separation in supersonic MHD turbulence}

OP Publishing

P Grete et al

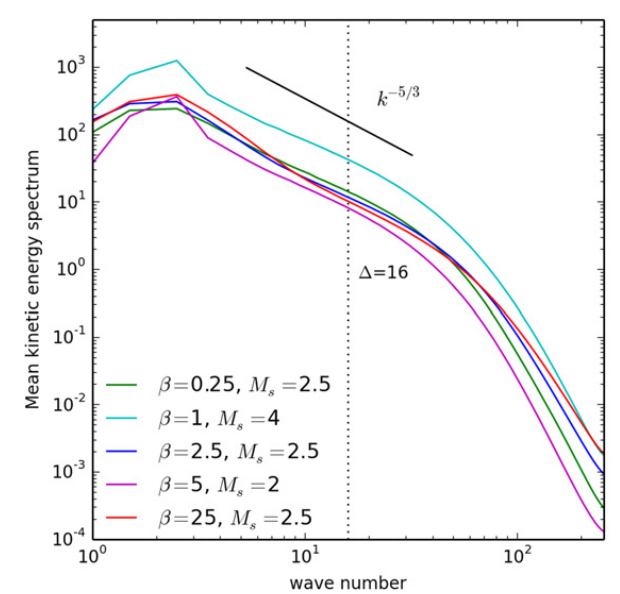

(a) kinetic energy

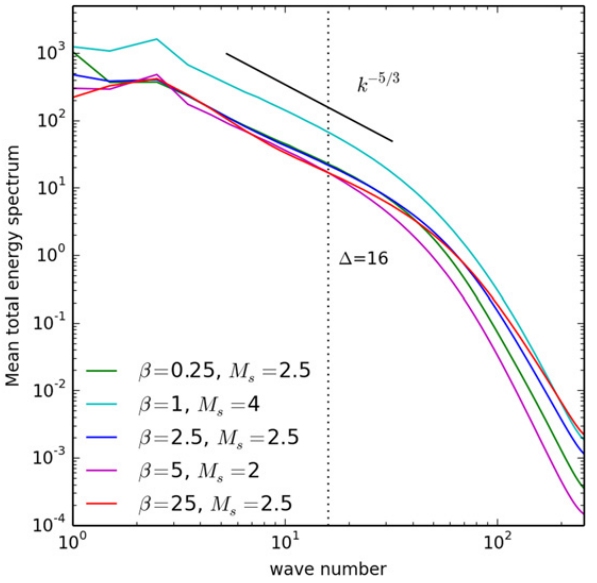

(b) kinetic + magnetic energy

Figure 1. Kinetic (a) and total (b) energy spectrum for each dataset, averaged over the time between $2 T$ and $5 T$. The kinetic energy is calculated from the Fourier transform of $\sqrt{\rho} \boldsymbol{u}$.

$\Sigma^{\mathcal{E}}=\mathcal{E} \cdot \overline{\boldsymbol{J}}$, respectively (see appendix of [10] for the detailed SGS energy equation). Here, we substitute (1) and (2) to obtain exact fluxes $\Sigma \square$ and match these to model fluxes $\widehat{\Sigma}^{\square}$ that employ the corresponding closures. For example, in the case of the eddy-viscosity closure for the deviatoric turbulent Reynolds stress tensor we compare the exact flux

$$
\Sigma^{\mathrm{u} \star}=\tau_{i j}^{\mathrm{u} *} \widetilde{S}_{i j}=\bar{\rho}\left(\left(\widetilde{u_{i} u_{j}}-\widetilde{u}_{i} \tilde{u}_{j}\right)-\frac{1}{3} \delta_{i j}\left(\widetilde{u_{k} u_{k}}-\widetilde{u}_{k} \tilde{u}_{k}\right)\right) \widetilde{S}_{i j}
$$

with the model flux

$$
\widehat{\Sigma}^{\mathrm{u} *}=\hat{\tau}_{i j}^{\mathrm{u} *} \widetilde{S}_{i j}=-\nu^{\mathrm{u}} \bar{\rho}\left|\widetilde{S^{*}}\right|^{2}=-C_{\nu}^{\mathrm{u}} \Delta\left(\bar{\rho} E_{\mathrm{sgs}}^{\mathrm{u}}\right)^{1 / 2}\left|\widetilde{S^{*}}\right|^{2}
$$

On the one hand, the comparison involves the determination of the constant (in space and time), dimensionless closure coefficients $C_{\square}^{\square}$. They are computed individually for each snapshot by minimizing the error between $\Sigma^{\square}$ and $\widehat{\Sigma}^{\square}$ in the least-square sense. This allows to further test the constancy of the coefficients with respect to time and plasma parameters. On the other hand, the general performance of the closure is gauged by computing the Pearson correlation coefficient of $\Sigma^{\square}$ and $\widehat{\Sigma^{\square}}$, where the obtained closure coefficients are substituted in.

Several assumptions should be pointed out concerning this validation technique. Firstly, the simulation data we have available for comparison fall short of realistic astrophysical parameters, e.g. with regards to Reynolds numbers and resolution. In that sense, it would be interesting to use higher resolution direct numerical simulation data or three-dimensional observations or experimental results. The problem is that experimental data for supersonic compressible turbulent plasmas are not available and obtaining realistic Reynolds numbers is computationally challenging for astrophysical parameters. However, as seen from figure 1 , the data we have are sufficiently well resolved for our analysis. Secondly, in choosing the SGS energy flux $\Sigma$, as a diagnostic variable, we implicitly assume that in the context of homogeneous and isotropic turbulence the turbulent transport (encoded by terms of the form $\nabla \cdot(\tilde{u} \cdot \tau)$ and $\nabla \cdot(\bar{B} \times \mathcal{E})$ ) averages out to zero on subgrid scales. This assumption can nevertheless be easily relaxed by incorporating further diagnostic variables. Finally, we have focused on the SGS energy since it increases monotonically with the strength of turbulence regardless of the type of turbulence (e.g. compressive or solenoidal, weak or strong, etc). As an extension, the other two quadratic MHD invariants- the magnetic helicity and cross-helicity, may further highlight distinct turbulence properties present in particular flow configurations. These should be kept in mind as further avenues of investigation, once a preferred closure has been identified by the described validation technique. 


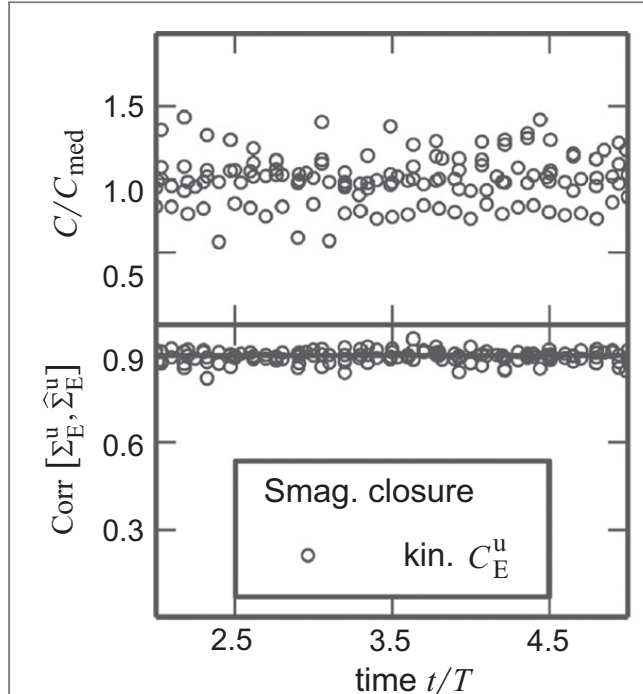

(a) kinetic SGS energy

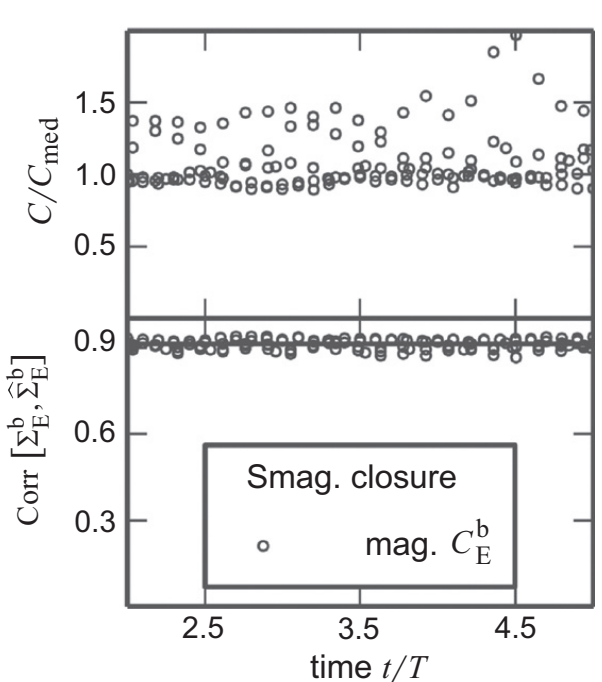

(b) magnetic SGS energy

Figure 2. Model coefficient values (top panels) and correlations (bottom panels with—-median) from fitting model energy flux $\widehat{\Sigma_{\mathrm{E}}^{\square}}$ $=\widehat{\tau}_{i j} \widetilde{S}_{i j}$ to exact flux for the isotropic parts of the SGS stress tensors. These are given by the respective energy model in the trace elements $\left(\tau_{i i}^{\square}=\frac{2}{3} E_{\mathrm{sgs}}^{\square}\right)$. Each panel contains the joint data of all simulations and each snapshot is represented by a marker. Values are given in table 1 .

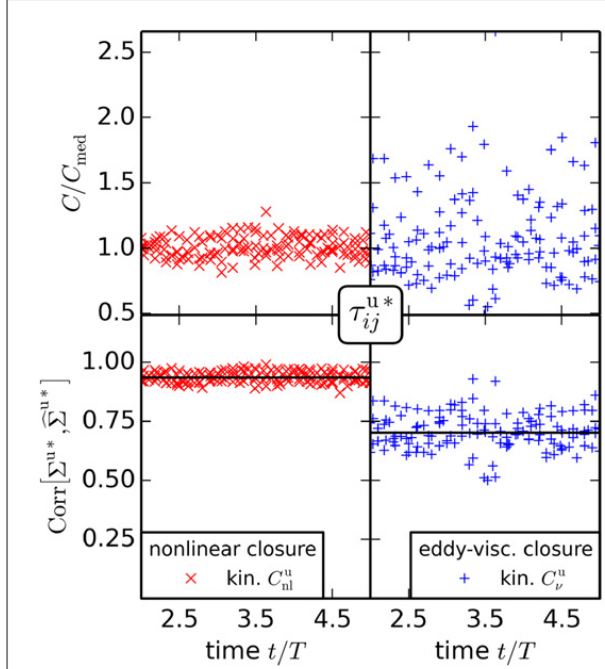

(a) traceless kinetic SGS stress tensor

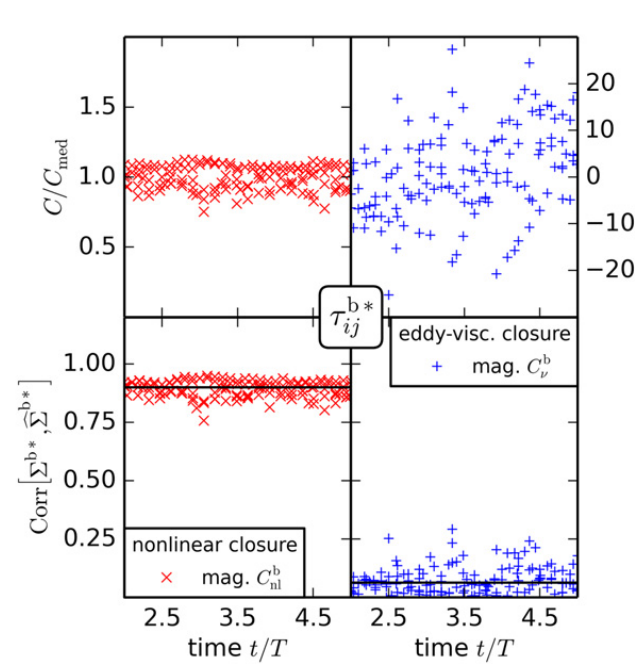

(b) traceless magnetic SGS stress tensor

Figure 3. Model coefficient values (top panels) and correlations (bottom panels with—-median) from fitting model energy flux $\widehat{\Sigma}^{\square}$ $=\widehat{\tau}_{i j}^{\square} \widetilde{S}_{i j}$ to exact flux for the nonlinear closure (left panels) and eddy-viscosity closure (right panels). Each panel contains the joint data of all simulations and each snapshot is represented by a marker. Values are given in table 1.

\section{Results}

The fitting results for the SGS stress tensors' energy flux are given in figure 2 for the isotropic components and figure 3 for the deviatoric components.

The isotropic parts of $\tau^{\mathrm{u}}$ and $\tau^{\mathrm{b}}$ are given by the SGS energies $\tau_{i i}^{\square}=\frac{2}{3} E_{\mathrm{sgs}}^{\square}$ from (3) and (7). Both the coefficient values of kinetic part (figure 2(a) top panel) and the magnetic part (figure 2(b) top panel), have a 


\section{Paper I: Nonlinear closures for scale separation in supersonic MHD turbulence}

OP Publishing New J. Phys. 17 (2015) 023070

Table 1. Model coefficient overview-coefficient value and energy flux correlation: median and bounds of the central $90 \%$ interval across all datasets.

\begin{tabular}{lccc}
\hline \multicolumn{1}{c}{ Model } & Coefficient & Value & $\operatorname{Corr}\left[\Sigma \square, \widehat{\Sigma}^{\square}\right]$ \\
\hline Smagorinsky & $C_{\mathrm{E}}^{\mathrm{u}}$ & $0.056_{-0.015}^{+0.016}$ & $0.90_{-0.04}^{+0.024}$ \\
& $C_{\mathrm{E}}^{\mathrm{b}}$ & $0.075_{-0.007}^{+0.034}$ & $0.91_{-0.04}^{+0.021}$ \\
\hline eddy-viscosity & $C_{\nu}^{\mathrm{u}}$ & $0.061_{-0.019}^{+0.045}$ & $0.70_{-0.11}^{+0.13}$ \\
& $C_{\nu}^{\mathrm{b}}$ & $-0.002_{-0.03}^{+0.029}$ & $0.06_{-0.06}^{+0.14}$ \\
\hline nonlinear & $C_{\mathrm{n}}^{\mathrm{u}}$ & $0.68_{-0.09}^{+0.09}$ & $0.94_{-0.04}^{+0.04}$ \\
& $C_{\mathrm{nn}}^{\mathrm{b}}$ & $0.77_{-0.12}^{+0.08}$ & $0.90_{-0.07}^{+0.04}$ \\
& $C_{\mathrm{nl}}^{\mathcal{E}}$ & $0.12_{-0.024}^{+0.013}$ & $0.79_{-0.17}^{+0.07}$ \\
\hline$\alpha-\beta-\gamma$ & $C_{\alpha}^{\mathcal{E}}$ & $0.0007_{-0.0016}^{+0.0010}$ & \\
& $C_{\beta}^{\mathcal{E}}$ & $0.020_{-0.005}^{+0.009}$ & $0.58_{-0.16}^{+0.06}$ \\
& $C_{\gamma}^{\mathcal{E}}$ & $-0.005_{-0.045}^{+0.067}$ & \} \\
\hline
\end{tabular}

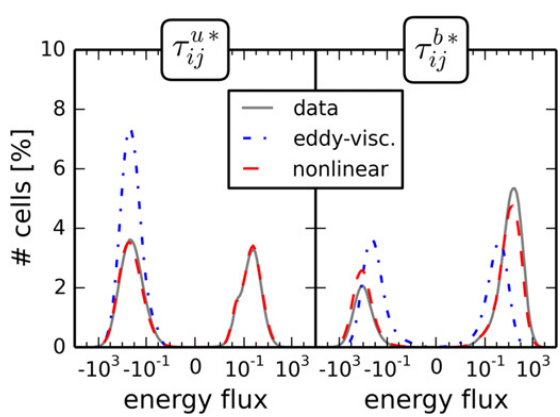

Figure 4. Representative snapshot (ENzo sim. with $\beta_{\mathrm{p}}=2.5$ at $t=4.44 T$ ) of the energy flux $\Sigma^{\square}=\tau_{i j}^{\square} S_{i j}$ distribution within the simulation box.

small spread within a factor of two across time and all simulations. Furthermore, closure and data are highly correlated (bottom panels) with a median correlation coefficient of 0.90 and 0.91 , respectively. More detailed numerical values of these and all following coefficients and correlations are listed in table 1 .

The differences in the deviatoric parts $\hat{\tau}^{\mathrm{u} *}$ in figure $3(\mathrm{a})$ and $\hat{\tau}^{\mathrm{b} *}$ in figure $3(\mathrm{~b})$ between the nonlinear and the eddy-viscosity closures are apparent. While our nonlinear closure exhibits approximately constant coefficient values and correlations over time in all simulations, the kinetic eddy-viscosity closure shows a correlation weaker by $\approx 0.2$ and bigger spread in coefficient values. Moreover, the magnetic eddy-viscosity closure is effectively uncorrelated with the simulation data and the coefficients can even switch sign at different times. The performance of the different closures can be understood from figure 4 , where we plot the energy flux distributions $\Sigma^{\mathrm{u} \star}$ and $\Sigma^{\mathrm{b} *}$ for a single snapshot. A negative flux $\Sigma^{\mathrm{u} \star}<0$ corresponds to a forward energy cascade- the transfer of energy from resolved to subgrid scales, because $\Sigma^{\mathrm{u} *}$ appears as a sink term in the SGS kinetic and magnetic energy evolution equations and as a source term in the respective resolved energy equations. Conversely, a positive flux corresponds to an inverse energy cascade, i.e. transport of energy from subgrid to resolved spatial scales. The general distribution of the actual fluxes in figure 4 is representative for all snapshots. The kinetic SGS energy fluxes are globally almost 1:1 in both directions of the turbulent cascade with a slight tendency towards the forward cascade. However, the forward cascade is about 3-10 times stronger depending on the parameters as indicated by the position of the peaks in the distribution. For this reason, the kinetic eddy-viscosity closure shows a moderate correlation even though it captures only the forward energy cascade-from large to small scales. In fact, since under the eddy-viscosity hypothesis the kinetic SGS energy flux has the form $\widehat{\Sigma}^{\mathrm{u} \star}=-\nu^{\mathrm{u}} \bar{\rho}\left|\tilde{S}^{\star}\right|^{2}$, see (9), any model in which the eddy-viscosity $\nu^{\mathrm{u}}$ has a definite signature with respect to space cannot reproduce a bi-directional energy cascade that is well represented by the nonlinear closure. In contrast to the kinetic SGS energy flux, the global magnetic flux clearly has a preferred direction. Depending on the parameters, between 60 and $80 \%$ of cells have a positive SGS magnetic flux indicating energy 


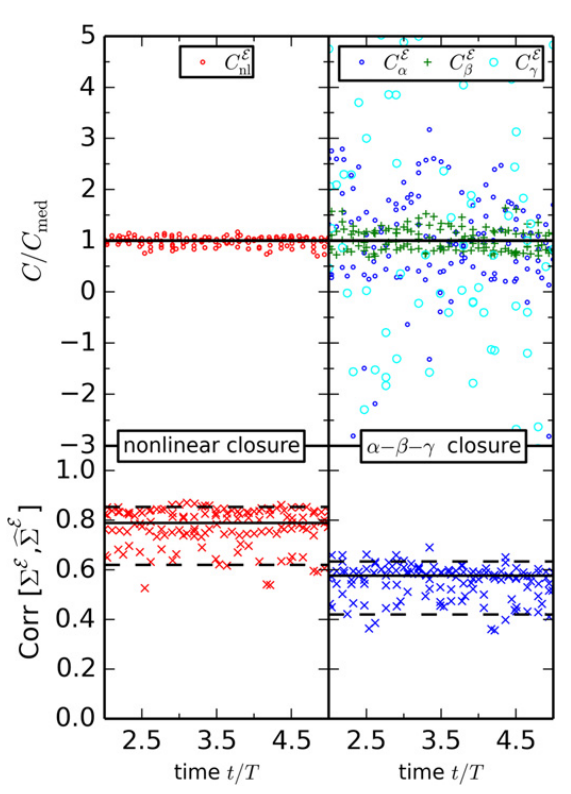

Figure 5. Model coefficient values (top panels) normalized to the sample median (一) and the corresponding Pearson correlation coefficients (bottom panels) with $90 \%$ central interval (- -) for the nonlinear closure (left panels) and the reference closure (right panels) from energy flux fitting for $\widehat{\mathcal{E}}$. Each panel contains the joint data of all simulations and each snapshot is represented by a marker. Values are listed in table 1.

transfer from large to small scales. Nevertheless, the difference in strength is less pronounced as the overall forward flux is about two times stronger than the backward one. Again, these properties are well captured by the proposed nonlinear closure whereas the magnetic eddy-viscosity closure is poorly correlated in both strength and magnitude.Finally, it should be noted, that the nonlinear closures also work very well with the Smagorinsky energy closure. Exchanging the exact expressions $E_{\text {sgs }}^{\square}$ in (4) and (5) with $\widehat{E}_{\text {sgs }}^{\square}$ only slightly reduces the correlations $(\max 5 \%)$ and the coefficients remain constant up to the second significant figure (not plotted here).

Moving on to the EMF, the nonlinear closure outperforms the traditional $\alpha-\beta-\gamma$ closure in almost all datasets, maintaining a constant coefficient with a median correlation of 0.79 (figure 5). The traditional closure exhibits consistently weaker correlations, despite the increased flexibility of three free coefficients. Only $C_{\beta}^{\mathcal{E}}$, related to the turbulent resistivity term in the EMF, is approximately constant, whereas $C_{\alpha}^{\mathcal{E}}$ and $C_{\gamma}^{\mathcal{E}}$ fail to maintain steady values or consistent signature. The reason for the consistently better correlations of the nonlinear closure is hinted at in figure 6. This probability density plot of the local alignment between $\mathcal{E}$ and $\widehat{\mathcal{E}}$ demonstrates that the traditional closure is almost randomly aligned (flat distribution) whereas the nonlinear closure approaches the desired $\delta$-distribution at $0^{\circ}$.

\section{Conclusions and outlook}

In summary, we have proposed a set of constant coefficient closures for the SGS stress and EMF in the filtered MHD equations and conducted a priori tests. The tests we performed do show that the new nonlinear closures perform significantly better than traditional, phenomenological closures with respect to both structural and functional diagnostics. The tests consist of filtering ENzO and FLASHv4 simulations of homogeneous, isotropic turbulence and comparing the resulting SGS terms to their respective closures (dependent only on the filtered fields). All quantities are compared via their contributions to the $S G S$ energy flux $\Sigma^{\square}$, where the closure coefficients are computed by individual least-square fitting. In addition, the alignment for the EMF vector is investigated. All new coefficients correlate well with the data. They are constant over time and as a direct consequence the proposed closures may be implemented in large-eddy simulations without the need for a computationally expensive dynamical procedure which computes the coefficient values at run time. In addition, the coefficients remain constant across simulation runs from two different codes and a wide range of plasma parameters, suggesting that the proposed closures capture an underlying physical mechanism at work in highly 


\section{Paper I: Nonlinear closures for scale separation in supersonic MHD turbulence}

OP Publishing

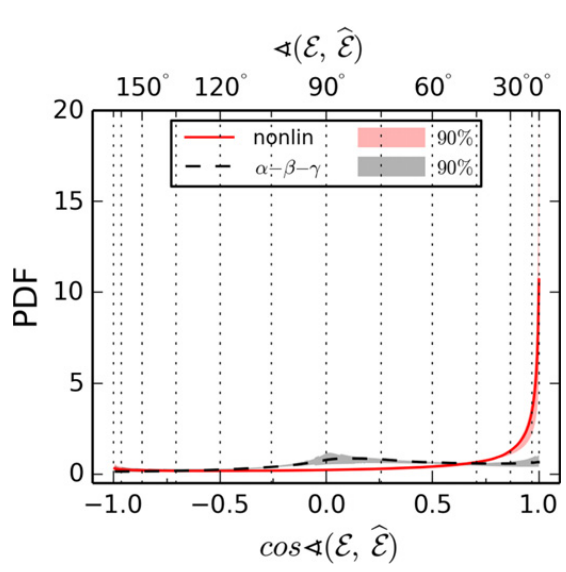

Figure 6. Probability density plot of the local (cell-by-cell) alignment between $\mathcal{E}$ and $\widehat{\mathcal{E}}$. The lines designate the median PDF across all times and datasets for the nonlinear closure (red-) and the $\alpha-\beta-\gamma$ closure (gray - -). The shaded regions designate the central $90 \%$ interval across all times and datasets.

compressible turbulent plasma flows. Moreover, the new closures successfully represent the turbulent magnetic pressure, reproduce the bi-directional energy cascade and are well aligned with the EMF. We recognize the slightly lower correlation of the nonlinear closure in the EMF than in the SGS stress counterpart, suggesting small room for improvement.

Nevertheless, the performance improvement over the traditional closures already supports the implementation and validation of the new closures in an SGS model for large-eddy simulations of compressible turbulent plasma flows. These simulations would then allow us to infer the effect of the proposed model on the large scale flow in practice. Potential applications include accretion disks [32], star-forming magnetized clouds $[33,34]$ and plasmas on cosmological scales [35-40].

\section{Acknowledgments}

PG worked on section 3 and conducted the Enzo simulations. DV worked on section 2. The analysis framework is a joint development by PG and DV. PG acknowledges financial support by the International Max Planck Research School for Solar System Science at the University of Göttingen. DV, WS and DS acknowledge research funding by the Deutsche Forschungsgemeinschaft (DFG) under grant SFB 963/1, project A15. CF is grateful for funding provided by the ARC (grants DP130102078 and DP150104329). We gratefully acknowledge the Jülich Supercomputing Centre (grant hhd20), the Leibniz Rechenzentrum and the Gauss Centre for Supercomputing (grant pr32lo), the Partnership for Advanced Computing in Europe (PRACE grant pr98mu), and the Australian National Computing Infrastructure (grant ek9). The software used in this work was in part developed by the DOE-supported Flash Center for Computational Science at the University of Chicago.

\section{References}

[1] Goldstein M L, Roberts D A and Matthaeus W H 1995 Annu. Rev. Astron. Astrophys. 33 283-326

2] Mac Low M M and Klessen R S 2004 Rev. Mod. Phys. 76 125-94

[3] Elmegreen B G and Scalo J 2004 Annu. Rev. Astron. Astrophys. 42 211-73

[4] Cooper C Metal 2014 Phys. Plasmas 21013505

[5] Schmidt W 2014 Living Rev. Comput. Astrophys. submitted arXiv:1404.2483

[6] Garnier E, Adams N and Sagaut P 2009 Large Eddy Simulation for Compressible Flows (Dordrecht: Springer)

[7] Müller W C and Carati D 2002 Phys. Plasmas $9824-34$

[8] Theobald M L, Fox P A and Sofia S 1994 Phys. Plasmas 13016-32

[9] Chernyshov A A, Karelsky K V and Petrosyan A S 2014 Phys.—Usp. 57 421-52

[10] Miki K and Menon S 2008 Phys. Plasmas 15072306

[11] Balarac G, Kosovichev A G, Brugière O, Wray A A and Mansour N N 2010 Modeling of the subgrid-scale term of the filtered magnetic field transport equation Proc. of the Summer Program (NASA: Center for Turbulence Research, Stanford University) pp 503-12 (available at http://ctr.stanford.edu/Summer/SP10/8 06 balarac.pdf)

[12] Favre A 1983 Phys. Fluids 26 2851-63

[13] Chernyshov A A, Karelsky K V and Petrosyan A S 2007 Phys. Fluids 19055106

[14] Smagorinsky J 1963 Mon. Weather Rev. 9199 


\section{Paper I: Nonlinear closures for scale separation in supersonic MHD turbulence}

[15] Yoshizawa A 1990 Phys. Fluids B 2 1589-600

[16] Schmidt W and Federrath C 2011 Astron. Astrophys. 528 A106

[17] Woodward P R, Porter D H, Anderson S, Fuchs T and Herwig F 2006 J. Phys.: Conf. Ser. 46 370-84

[18] Bryan G Let al 2014 Astrophys. J. Suppl. Ser. 21119

[19] Fryxell B, Olson K, Ricker P, Timmes F X, Zingale M, Lamb D Q, MacNeice P, Rosner R, Truran J W and Tufo H 2000 Astrophys. J. Suppl. Ser. $131273-334$

[20] Waagan K, Federrath C and Klingenberg C 2011 J. Comput. Phys. 230 3331-51

[21] Federrath C, Chabrier G, Schober J, Banerjee R, Klessen R S and Schleicher D R G 2011 Phys. Rev. Lett. 107114504

[22] Federrath C, Schober J, Bovino S and Schleicher D R G 2014 Astrophys. J. 797 L19

[23] Dedner A, Kemm F, Kröner D, Munz C D, Schnitzer T and Wesenberg M 2002 J. Comput. Phys. 175645 - 673

[24] Schmidt W, Federrath C, Hupp M, Kern S and Niemeyer J C 2009 Astron. Astrophys. 494 127-45

[25] Federrath C, Roman-Duval J, Klessen R S, Schmidt W and Mac Low M M 2010 Astron. Astrophys. 512 A81

[26] Federrath C 2013 Mon. Not. R. Astron. Soc. $4361245-57$

[27] Galtier S and Banerjee S 2011 Phys. Rev. Lett. 107134501

[28] Banerjee S and Galtier S 2013 Phys. Rev. E 87 1-8

[29] Aluie H 2011 Phys. Rev. Lett. 106174502

[30] Aluie H 2013 Physica D 247 54-65

[31] Kitsionas Setal 2009 Astron. Astrophys. 508 541-60

[32] Clark P C, Glover S C O, Smith R J, Greif T H, Klessen R S and Bromm V 2011 Science 3311040

[33] Greif T H, Schleicher D R G, Johnson J L, Jappsen A K, Klessen R S, Clark P C, Glover S C O, Stacy A and Bromm V 2008 Proc. Int. Astron. Union 433

[34] Greif T H, Bromm V, Clark P C, Glover S C O, Smith R J, Klessen R S, Yoshida N and Springel V 2012 AIP Conf. Proc. 51 51-56

[35] Agertz O, Kravtsov A V, Leitner S N and Gnedin N Y 2013 Astrophys. J. 77025

[36] Devriendt Jet al 2010 Mon. Not. R. Astron. Soc. Lett. 403 84-88

[37] Vazza F, Brüggen M, Gheller C and Wang P 2014 Mon. Not. R. Astron. Soc. $4453706-22$

[38] Schleicher D, Latif M, Schober J, Schmidt W, Bovino S, Federrath C, Niemeyer J, Banerjee R and Klessen R 2013 Astron. Nachr. 334 531-6

[39] Latif M A, Schleicher D R G, Schmidt W and Niemeyer J 2013 Mon. Not. R. Astron. Soc. 432 668-78

[40] Latif M A, Schleicher D R G and Schmidt W 2014 Mon. Not. R. Astron. Soc. 440 1551-61 



\section{Paper II: A nonlinear structural subgrid-scale closure for compressible MHD. I. Derivation and energy dissipation properties}

The paper originally appeared as Dimitar G. Vlaykov, Philipp Grete, Wolfram Schmidt, and Dominik R. G. Schleicher. A nonlinear structural subgrid-scale closure for compressible MHD. I. Derivation and energy dissipation properties. Physics of Plasmas, 23 (6):062316, 2016. doi:10.1063/1.4954303.

The research was jointly designed and planned by all authors. PG was actively involved in the discussions leading to the derivation of the new closures. PG supported the writing of the final paper by DGV with comments and suggestions at the time of preparing a draft and during the revision. PG checked the calculations and derivations. 


\title{
A nonlinear structural subgrid-scale closure for compressible MHD. I. Derivation and energy dissipation properties
}

\author{
Dimitar G. Vlaykov, ${ }^{1,2, a)}$ Philipp Grete, ${ }^{1,3}$ Wolfram Schmidt, ${ }^{4}$ and Dominik R. G. Schleicher ${ }^{5}$ \\ ${ }^{1}$ Institut für Astrophysik, Universität Göttingen, Friedrich-Hund-Platz 1, D-37077 Göttingen, Germany \\ ${ }^{2}$ Max-Planck-Institut für Dynamik und Selbstorganisation, Am Faßberg 17, D-37077 Göttingen, Germany \\ ${ }^{3}$ Max-Planck-Institut für Sonnensystemforschung, Justus-von-Liebig-Weg 3, D-37077 Göttingen, Germany \\ ${ }^{4}$ Hamburger Sternwarte, Universität Hamburg, Gojenbergsweg 112, D-21029 Hamburg, Germany \\ ${ }^{5}$ Departamento de Astronomía, Facultad Ciencias Físicas y Matemáticas, Universidad de Concepción, \\ Av. Esteban Iturra s/n Barrio Universitario, Casilla 160-C, Chile
}

(Received 5 February 2016; accepted 12 May 2016; published online 28 June 2016)

Compressible magnetohydrodynamic (MHD) turbulence is ubiquitous in astrophysical phenomena ranging from the intergalactic to the stellar scales. In studying them, numerical simulations are nearly inescapable, due to the large degree of nonlinearity involved. However, the dynamical ranges of these phenomena are much larger than what is computationally accessible. In large eddy simulations (LESs), the resulting limited resolution effects are addressed explicitly by introducing to the equations of motion additional terms associated with the unresolved, subgrid-scale dynamics. This renders the system unclosed. We derive a set of nonlinear structural closures for the ideal MHD LES equations with particular emphasis on the effects of compressibility. The closures are based on a gradient expansion of the finite-resolution operator [W. K. Yeo (CUP, 1993)] and require no assumptions about the nature of the flow or magnetic field. Thus, the scope of their applicability ranges from the sub- to the hyper-sonic and -Alfvénic regimes. The closures support spectral energy cascades both up and down-scale, as well as direct transfer between kinetic and magnetic resolved and unresolved energy budgets. They implicitly take into account the local geometry, and in particular, the anisotropy of the flow. Their properties are a priori validated in Paper II [P. Grete et al., Phys. Plasmas 23, 062317 (2016)] against alternative closures available in the literature with respect to a wide range of simulation data of homogeneous and isotropic turbulence. Published by AIP Publishing. [http://dx.doi.org/10.1063/1.4954303]

\section{INTRODUCTION}

There is a great need for increased accuracy in numerical simulations involving turbulent flows of magnetized fluids in fields varying from engineering to astrophysics. In astrophysics, in particular, compressible magnetohydrodynamic (MHD) turbulence is an important ingredient in the solution of outstanding problems on many scales such as the generation and sustainment of galactic and super-galactic scale magnetic fields; ${ }^{3-5}$ the detailed process of star formation, including self-regulation and fragmentation; ${ }^{6-8}$ stellar convection in the interior and stellar atmospheres; ${ }^{9}$ accretion and protoplanetary discs, stellar ejecta, e.g., jets, winds, and outflows; ${ }^{10,11}$ and the dynamics of the solar tachocline, the solar wind, and the solar corona. ${ }^{12-16}$ The dynamical range of these phenomena is usually much larger than what is computationally tractable. Numerically, this translates to unphysical dissipation and turbulence dynamics due to the limited resolution. For example, in finite-volume numerical schemes, it leads to enhanced dissipation. In large eddy simulations (LESs), ${ }^{17-20}$ this problem is tackled by directly solving only the evolution equations for the resolved fields. The contribution of the small under and unresolved scales (i.e., the scales which are badly contaminated by numerical noise or simply unrepresented) on them has to be incorporated via explicit

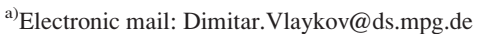

modeling. Formally, these scales are identified by the introduction of a finite resolution operator, in effect a low-pass filter. Large eddy simulations are typically used with grid-based numerical schemes, e.g., based on finite-differences or finitevolumes. As such the grid-scale can be taken to be the filter scale and hence the terms responsible for the small-scale effects are known as subgrid-scale (SGS) terms.

The magnetohydrodynamic LES equations are obtained by applying a finite resolution operator to the MHD equations. It can be shown that this operator can be expressed as a convolution with a low-pass filter kernel. There are several comprehensive reviews of the formalism and its application to hydrodynamics ${ }^{17,18,20}$ and MHD. ${ }^{21}$ Applying the formalism with a static, homogeneous and isotropic kernel $G$ with a constant grid-scale (which can be used to represent the commonly used grid-based numerical schemes in physical or spectral space) under periodic boundary conditions to the ideal MHD equations results in the following equations for the large-scale fields:

$$
\frac{\partial \bar{\rho}}{\partial t}+\nabla \cdot(\bar{\rho} \widetilde{\mathbf{u}})=0
$$

$$
\frac{\partial \bar{\rho} \widetilde{\mathbf{u}}}{\partial t}+\nabla \cdot(\bar{\rho} \widetilde{\mathbf{u}} \otimes \widetilde{\mathbf{u}}-\overline{\mathbf{B}} \otimes \overline{\mathbf{B}})+\nabla\left(\bar{P}+\frac{\bar{B}^{2}}{2}\right)=-\nabla \cdot \tau,
$$




\section{Paper II: A nonlinear structural subgrid-scale closure for compressible MHD. I. Derivation and energy dissipation properties}

$$
\frac{\partial \overline{\mathbf{B}}}{\partial t}-\nabla \times(\widetilde{\mathbf{u}} \times \overline{\mathbf{B}})=\nabla \times \mathcal{E} .
$$

Here, a large scale, filtered field is denoted by an overbar. For instance, the large scale component of the pressure $P$ is given by a convolution with the filter kernel $G$, i.e., $\bar{P}$ $=G * P$ and similarly for the filtered density $\bar{\rho}$ and the magnetic field $\overline{\mathbf{B}}$, which incorporates $\sqrt{4 \pi}$ in the chosen notation. The treatment of the pressure term is beyond the scope of this work due to the wide array of possible equations of state used to close the MHD system. Nevertheless, briefly, if the equation of state is linear in the primary fields (e.g., in isothermal conditions), the pressure does not lead to any SGS contributions.

The tilde denotes a mass-weighted (also known as Favre) filtered field, ${ }^{22}$ e.g., the Favre-filtered velocity field $\tilde{u}=\overline{\rho \mathbf{u}} / \bar{\rho}$. Using $\tilde{u}$ as a primary quantity precludes the introduction of SGS terms in the mass conservation equation. Additionally, it fits well with physical-space-based compressible schemes, where often the momentum $\rho \mathbf{u}$ is evolved as the primary quantity instead of the velocity $\mathbf{u}$. The momentum and induction equations contain two new, SGS terms, $\nabla \cdot \tau$ and $\nabla \times \mathcal{E}$, which will occupy the focus of this article. They are simply the commutators between the finite resolution operator and the nonlinearities of the respective MHD equations. Thus they carry information about the interactions across the filter scale. Analytically, they are given by

$$
\mathcal{E}=\overline{\mathbf{u} \times \mathbf{B}}-\tilde{\mathbf{u}} \times \overline{\mathbf{B}},
$$

and

$$
\tau_{i j}=\tau_{i j}^{u}-\tau_{i j}^{b}+\frac{1}{2} \tau_{k k}^{b} \delta_{i j},
$$

with

$$
\tau_{i j}^{u}=\bar{\rho}\left(\widetilde{u_{i} u_{j}}-\tilde{u}_{i} \tilde{u}_{j}\right), \quad \tau_{i j}^{b}=\left(\overline{B_{i} B_{j}}-\bar{B}_{i} \bar{B}_{j}\right),
$$

where the Einstein summation convention is assumed. The tensor $\tau$ is known as the SGS stress and can be decomposed into kinetic and magnetic components, SGS Reynolds stress $\tau^{u}$ and SGS Maxwell stress $\tau^{b}$, respectively. The (pseudo-) vector $\mathcal{E}$ is known as the electromotive force (EMF). They carry information about the subgrid-scales via the terms $\overline{\mathbf{u} \times \mathbf{B}}, \widetilde{u_{i} u_{j}}$, and $\overline{B_{i} B_{j}}$ and thus cannot be explicitly expressed only in terms of large scale fields. This renders the system of equations (1) unclosed. The evolution equations of the SGS terms ${ }^{17}$ involve new, higher order unknown terms. This continues to build an infinite hierarchy. This is the LES aspect of the well-known turbulence closure problem.

The resolved, i.e., large scale, energies and crosshelicity are defined as

$$
E_{\mathrm{res}}^{\mathrm{u}}=\frac{1}{2} \bar{\rho} \widetilde{\mathbf{u}}^{2}, E_{\mathrm{res}}^{\mathrm{b}}=\frac{1}{2} \overline{\mathbf{B}}^{2}, E_{\mathrm{res}}=E_{\mathrm{res}}^{\mathrm{u}}+E_{\mathrm{res}}^{\mathrm{b}},
$$

and

$$
W_{\text {res }}=\tilde{\mathbf{u}} \cdot \overline{\mathbf{B}} .
$$

Their evolution equations are obtained in the classical manner from the corresponding primary LES equations. ${ }^{23}$ For ideal MHD they can be written as

$$
\begin{gathered}
\frac{\partial}{\partial t} E_{\mathrm{res}}^{\mathrm{u}}+\nabla \cdot\left(\widetilde{\mathbf{u}} E_{\mathrm{res}}^{\mathrm{u}}\right)+\widetilde{\mathbf{u}} \cdot \overline{\mathbf{B}} \times \overline{\mathbf{J}}+\widetilde{\mathbf{u}} \cdot \nabla \bar{P} \\
=-\widetilde{\mathbf{u}} \cdot(\nabla \cdot \tau), \\
\frac{\partial}{\partial t} E_{\mathrm{res}}^{\mathrm{b}}-\overline{\mathbf{B}} \cdot \nabla \times(\widetilde{\mathbf{u}} \times \overline{\mathbf{B}})=\overline{\mathbf{B}} \cdot \nabla \times \mathcal{E}, \\
\frac{\partial E_{\mathrm{res}}}{\partial t}+\nabla \cdot\left(\widetilde{\mathbf{u}} E_{\mathrm{res}}^{\mathrm{u}}+2 \widetilde{\mathbf{u}} E_{\mathrm{res}}^{\mathrm{b}}-\overline{\mathbf{B}} W_{\mathrm{res}}\right)+\widetilde{\mathbf{u}} \cdot \nabla \bar{P} \\
=\overline{\mathbf{B}} \cdot \nabla \times \mathcal{E}-\widetilde{\mathbf{u}} \cdot(\nabla \cdot \tau), \\
\frac{\partial}{\partial t} W_{\mathrm{res}}+\nabla \cdot\left(\widetilde{\mathbf{u}} W_{\mathrm{res}}-\frac{\overline{\mathbf{B}}}{\bar{\rho}} E_{\mathrm{res}}^{\mathrm{u}}\right)+\frac{\overline{\mathbf{B}}}{\bar{\rho}} \cdot \nabla \bar{P} \\
=\widetilde{\mathbf{u}} \cdot \nabla \times \mathcal{E}-\frac{\overline{\mathbf{B}}}{\bar{\rho}} \cdot(\nabla \cdot \tau),
\end{gathered}
$$

where $\overline{\mathbf{J}}=\nabla \times \overline{\mathbf{B}}$ is the resolved current density. Although the total energy and cross-helicity are ideal MHD invariants, their resolved counterparts, as defined above, are not, due to the SGS terms on the right hand side of Eqs. (6) and (7). The equations show that the SGS stress and EMF encode the entire transfer of energy and cross-helicity across the filter scale and truncating the SGS hierarchy at the level of $\tau$ and $\mathcal{E}$ closes these equations as well.

Various approaches have been developed to address the closure problem for hydrodynamics, ${ }^{17,18}$ in and astrophysical settings. ${ }^{20}$ Several models have also been extended to the case of magnetized fluids, ${ }^{24-26}$ some of them taking into account compressibility as well. ${ }^{21,27}$ They can be separated heuristically into structural and functional ones. Functional closures focus on the effect of the SGS terms on the resolved scales and are thus largely phenomenological. For instance, the eddy-viscosity (EV) models ${ }^{21}$ address the anomalous energy dissipation due to turbulence, while dynamo models ${ }^{28,29}$ address the generation and amplification of magnetic fields. Structural models try to mimic some aspect of the structure of the SGS terms, expecting that the desired effects on the large scale will follow automatically. Thus, they largely rely on the robustness of these aspects. In the self-similarity closures, ${ }^{21,30}$ for example, the main assumption is the self-similarity of turbulence in the inertial range. In that context, functional models are useful in situations in which the effect of the unresolved scales is well understood and quantified. Since in practice this is rarely the case for compressible MHD, and in the absence of extensive experimental data for calibration and validation, we proceed with the derivation of a nonlinear structural closure, which is based on the properties of the finite resolution operator, rather than turbulence itself. Thus, the MHD turbulence dynamics is not required to obey any strong assumptions, like scale-similarity, existence of an inertial range, energy cascade, etc. The resulting closure is closely related to a previously a priori validated one $^{27}$ but includes additional compressibility effects. The present paper focuses on the derivation of the new compressible MHD closure, the analytic description of its scope of applicability, and energy dissipation properties. A numerical validation of the closure is performed in Paper II $^{2}$ by a priori comparison to well-resolved numerical data, where it is found to outperform all closures with which it has been compared. 


\section{APPROXIMATE DECONVOLUTION}

As usual in the LES theory, the presented closure has its origins in incompressible hydrodynamics. In particular, it is a self-consistent extension of the Yeo-Bedford (YB) expansions $^{1,31}$ as applied to compressible MHD. Closures of this family have been recently applied to incompressible ${ }^{32-34}$ and compressible (supersonic) MHD ${ }^{23,27}$ turbulence with encouraging results. The same method has also been used to model the transport of a passive scalar. ${ }^{34}$ Here, we focus on the closure derivation and extend it to include so far unaccounted for compressibility effects.

For clarity, this section summarizes the original derivation $^{31}$ as applied to a Gaussian filter kernel and the incompressible MHD SGS terms. The Gaussian kernel can be represented by its Fourier transform, i.e., transfer function $\hat{G}$ given by

$$
\hat{G}(k)=\exp \left(-\Delta^{2} k^{2} /(4 \gamma)\right),
$$

with wavenumber $k$ and filter scale $\Delta$. It is infinitely differentiable, which renders it particularly suitable for analytical manipulation. It is also positive and therefore signature preserving. Thus under its action, the SGS counterparts of positive definite quantities like energy are also positive definite. ${ }^{17}$ Furthermore, by setting the width parameter $\gamma=6$, its first and second order moments match those of a box filter with the same filter scale $\Delta$.

The main idea of the YB expansion is to compute an approximation of the inverse filtering operator based on gradient expansion of the filter kernel $G$. This amounts to computing an approximation of the inverse Fourier transform of $1 / \hat{G}$. The first step is to perform a Taylor expansion of the transfer function and its inverse in terms of the filter scale $\Delta$, i.e.,

$$
\begin{gathered}
\hat{G}(\mathbf{k})=\sum_{n=0}^{\infty} \frac{(-1)^{n}}{n !}\left(\frac{\Delta^{2}}{4 \gamma} \mathbf{k}^{2}\right)^{n}, \\
\frac{1}{\hat{G}(\mathbf{k})}=\sum_{n=0}^{\infty} \frac{1}{n !}\left(\frac{\Delta^{2}}{4 \gamma} \mathbf{k}^{2}\right)^{n} .
\end{gathered}
$$

Applying the expansions to the test fields $\hat{f}$ and $\hat{\bar{f}}$, respectively, followed by an inverse Fourier transformation yields infinite series representations of the filter operator and its inverse in terms of gradient operators acting on the test fields

$$
\begin{gathered}
\bar{f}=G * f=\sum_{n=0}^{\infty} \frac{1}{n !}\left(\frac{\Delta^{2}}{4 \gamma} \nabla^{2}\right)^{n} f, \\
f=G^{-1} * \bar{f}=\sum_{n=0}^{\infty} \frac{(-1)^{n}}{n !}\left(\frac{\Delta^{2}}{4 \gamma} \nabla^{2}\right)^{n} \bar{f} .
\end{gathered}
$$

They are absolutely convergent and formally accurate at all orders, since the Gaussian kernel is infinitely differentiable and with unbounded support. In fact, it has been found ${ }^{35}$ that the series given in Eq. (11) converges for all canonical filters, and more generally, symmetry of the filtering kernel and nonnegativity of its transfer function are sufficient conditions for its convergence for a periodic band-limited field $f$. (The last condition is trivially satisfied in any numerical simulation.) It has also been suggested ${ }^{35}$ that qualitatively the convergence rate tends to decrease as the dissipative strength of the filter increases. In the case of the Gaussian filter, the same results hold for the forward expansion Eq. (12), as it differs from Eq. (11) only by an alternating sign.

To proceed note that the unknown components of the SGS stresses and the EMF are of the form $\overline{f g}$. Applying Eq. (11) to such an expression results in a series in terms of $(f g)$. As it is absolutely convergent, Eq. (12) can be applied separately to each $f$ and $g$ term of the series. The result can be simplified to

$$
\begin{aligned}
\overline{f g}= & \bar{f} \bar{g}+2 a \bar{f}_{, k} \bar{g}_{, k}+\frac{1}{2 !}(2 a)^{2} \bar{f}_{, k l} \bar{g}_{, k l} \\
& +\frac{1}{3 !}(2 a)^{3} \bar{f}_{, k l m} \bar{g}_{, k l m}+O\left(a^{4} \nabla^{8}\right),
\end{aligned}
$$

as given in Eq. (5.21) of Yeo. ${ }^{31}$ Here, a comma is used to represent differentiation with respect to a co-ordinate and $a=\Delta^{2} /(4 \gamma)$. The coefficients in the expansions are given in terms of moments of the transfer function and its inverse. This relationship comes from the orthogonality of the terms in the Fourier expansion and thus holds for any filter kernel for which the expansion exists. There is a closed form expression $^{36}$ for the coefficients in Eq. (13) for a symmetric filter kernel $G$ with infinitely differentiable transfer function-they are given by the Taylor coefficients of the function $F(f, g)=G(-i(f+g)) /(G(-i f) G(-i g))$. Moreover, since any symmetric filter has a real transfer function, only the even order coefficients are non-zero. This symmetry has a fundamental impact on the form of the terms in the expansion as well, namely, each field is differentiated at most once with respect to a co-ordinate.

Recall that for $\gamma=6$ the Gaussian and box filter kernels have identical first and second moments. Therefore, with this parameter choice Eq. (13) is also valid for a box filter up to second order. Furthermore, since all moments of a Gaussian function can be expressed in terms of its second order moment, here $(2 a)$, it is the only parameter which can appear in Eq. (13).

Applying Eq. (13) to the SGS terms in the incompressible MHD equations is sufficient to completely close them

$$
\begin{aligned}
\overline{u_{i} u_{j}}-\bar{u}_{i} \bar{u}_{j} & =2 a \bar{u}_{i, k} \bar{u}_{j, k}, \\
\overline{B_{i} B_{j}}-\bar{B}_{i} \bar{B}_{j} & =2 a \bar{B}_{i, k} \bar{B}_{j, k}, \\
(\overline{\mathbf{u} \times \mathbf{B}}-\overline{\mathbf{u}} \times \overline{\mathbf{B}})_{i} & =2 a \epsilon_{i j k} \bar{u}_{j, l} \bar{B}_{k, l} .
\end{aligned}
$$

It should be noted that the resulting closures have been reached by alternative routes in hydrodynamic LES. The tensor-diffusivity models, ${ }^{37-39}$ for instance, use Taylor expansions of the SGS terms with respect to the turbulent fluctuations (e.g., $\mathbf{u}^{\prime}=\mathbf{u}-\tilde{\mathbf{u}}$ ) or the entire (unfiltered) fields (e.g., u). These derivations however are questionable as they require smoothness of the small scales. ${ }^{40}$ Another alternative, originally designed for image processing ${ }^{41}$ is given by approximate deconvolution closures. ${ }^{18,42-47}$ They are again based on the truncation of an infinite series to reconstruct the inverse of the filtering operator. However, in this approach, 


\section{Paper II: A nonlinear structural subgrid-scale closure for compressible MHD. I. Derivation and energy dissipation properties}

the series is not necessarily convergent and truncating at the optimal order is critical. The results of both approaches for a Gaussian filter agree with Eq. (13) up to second order. ${ }^{18}$ The different motivations and derivation are revealed only at higher orders.

\section{COMPRESSIBLE EXTENSIONS}

To apply the presented derivation self-consistently to the compressible Reynolds SGS stress and EMF, as defined in Eq. (2), the compressibility effects onto the massweighted large scale velocity have to be taken further into account. The issue can be addressed from several viewpoints. On the one hand, one can dispense with the massweighted filtering operator altogether, and re-substitute $\tilde{f} \bar{\rho}=\overline{f \rho}$ in the relevant SGS terms. This requires that an additional SGS term $\overline{\rho u_{i}}-\bar{\rho} \bar{u}_{i}$ is introduced in the continuity equation, and that the EMF and the Reynolds SGS stress are re-defined. The complexity of the Reynolds SGS stress $\tau^{\mathrm{u}}$ is formally increased, as it now contains an unclosed product of three fields, i.e., $\overline{\rho u_{i} u_{j}}$. Nevertheless, the derivation outlined above still holds. Applying Eqs. (11) and (12) to a general term of third order leads to (as given in Eq. (5.23) of $\mathrm{Yeo}^{31}$ )

$$
\begin{aligned}
\overline{f g h}= & \bar{f} \bar{g} \bar{h}+2 a\left(\bar{f}_{, k} \bar{g}_{, k} \bar{h}+\bar{f}_{, k} \bar{g} \bar{h}_{, k}+\bar{f}_{\bar{g}_{, k}} \bar{h}_{, k}\right) \\
& +\frac{1}{2 !}(2 a)^{2}\left(\bar{f}_{, k l} \bar{g}_{, k l} \bar{h}+\bar{f}_{, k l} \bar{g} \bar{h}_{, k l}+\bar{f}_{\bar{g}} \bar{g}_{, k l} \bar{h}_{, k l}\right. \\
& \left.+2 \bar{f}_{, k} \bar{g}_{, k l} \bar{h}_{, l}+2 \bar{f}_{, k} \bar{g}_{, l} \bar{h}_{, k l}+2 \bar{f}_{, k l} \bar{g}_{, k} \bar{h}_{, l}\right) \\
& +O\left(a^{3} \nabla^{6}\right) .
\end{aligned}
$$

To first order in $a$, this technique leads to the following results for the primary SGS terms:

$$
\begin{aligned}
& \overline{\rho u_{i}}-\bar{\rho} \bar{u}_{i}=2 a \bar{\rho}_{, k} \bar{u}_{i, k} \\
& \overline{\rho u_{i} u_{j}}-\bar{\rho} \bar{u}_{i} \bar{u}_{j}=2 a \bar{\rho} \bar{u}_{i, k} \bar{u}_{j, k}+2 a \bar{\rho}_{, k}\left(\bar{u}_{i, k} \bar{u}_{j}+\bar{u}_{i} \bar{u}_{j, k}\right) \text {, } \\
& \overline{B_{i} B_{j}}-\bar{B}_{i} \bar{B}_{j}=2 a \bar{B}_{i, k} \bar{B}_{j, k} \\
& (\overline{\mathbf{u} \times \mathbf{B}}-\overline{\mathbf{u}} \times \overline{\mathbf{B}})_{i}=2 a \epsilon_{i j k} \bar{u}_{j, l} \bar{B}_{k, l} .
\end{aligned}
$$

This constitutes a complete closure of the compressible MHD equations (barring pressure considerations). This approach is applicable for numerical schemes which evolve the velocity field, because only directly filtered fields are present. Even though such schemes are not frequently used to address highly compressible problems, such a model has been implemented in compressible hydrodynamics. ${ }^{48}$

On the other hand, for applications to compressible codes which treat the momentum as a primary quantity, e.g., using finite volume schemes, one needs to take into account the mass-weighted filtering operator. For a field $f$, it is given by $\tilde{f}=(G *(\rho f)) /(G * \rho)$. In the process of directly applying the outlined procedure to this operator, several fundamental challenges are encountered. The main obstacle is that since its filter kernel contains strongly fluctuating contributions (e.g., from the $G * \rho$ component), the Taylor expansion of its transfer function is not well-defined. Additionally, the existence of the inverse transfer function is not assured over an extended interval in spectral space.

\section{A. Simple compressible extension}

The simplest hypothesis which circumvents the complications outlined above would be to assume that even if the derivation is not valid for compressible MHD, its result still holds, i.e., to apply the map

$$
\overline{\mathbf{u}} \rightarrow \tilde{\mathbf{u}}
$$

to the incompressible closures Eq. (14). This would imply that the compressibility effects are implicitly taken into account by the change of operator. Qualitatively, this approach could be motivated by invoking the reduction of compressibility effects at smaller scales, ${ }^{49}$ but ultimately it is the simplest compressibility extension of Eq. (14). In fact, a previous a priori comparison ${ }^{27}$ with data from supersonic numerical simulations showed that this extension yields consistently higher correlation with the data than the other tested classical closures. However, while the results for the SGS stress were consistently high, the EMF closure exhibited a comparatively larger scatter. This difference can be explained by the self-consistent derivation of compressibility effects which follows.

\section{B. Primary compressible extension}

The goal is to obtain an expression of a simply filtered field in terms of the corresponding mass-weighted filtered field. Since mass-weighting applies to velocity-related fields, consider in particular, $\tilde{\mathbf{u}}=\overline{\mathbf{u} \rho} / \bar{\rho}$. Applying Eq. (13) to the right-hand side leads to

$$
\tilde{u}_{i}=\bar{u}_{i}+2 a y_{, k} \bar{u}_{i, k}+2 a^{2}\left(y_{, k l}+y_{, k} y_{, l}\right) \bar{u}_{i, k l}+O\left(a^{3}\right),
$$

where we denote for brevity the natural logarithm of the resolved density as $y=\ln \bar{\rho}$. As Eq. (18) represents an absolutely convergent series, under the same conditions as the original expansion Eq. (11), it can be rearranged to give

$$
\bar{u}_{i}=\tilde{u}_{i}-2 a y_{, k} \bar{u}_{i, k}-2 a^{2}\left(y_{, k l}+y_{, k} y_{, l}\right) \bar{u}_{i, k l}-O\left(a^{3}\right) .
$$

To this we can apply a recurrence technique. To second order in $a$ it gives

$$
\begin{aligned}
\bar{u}_{i}= & \tilde{u}_{i}-2 a y_{, k} \tilde{u}_{i, k}-2 a^{2}\left(\left(y_{, k l}-y_{, k} y_{, l}\right) \tilde{u}_{i, k l}-2 y_{, k} y_{, k l} \tilde{u}_{i, l}\right) \\
& -O\left(a^{3}\right) .
\end{aligned}
$$

This expression, along with Eqs. (13) and (15), can be applied to the definition of the SGS terms, Eq. (2), to obtain

$$
\begin{aligned}
\tau_{i j}^{\mathrm{u}}= & 2 a \bar{\rho} \tilde{u}_{i, k} \tilde{u}_{j, k}+2 a^{2} \bar{\rho}\left(\tilde{u}_{i, k l} \tilde{u}_{j, k l}-2 y_{, k l} \tilde{u}_{i, k} \tilde{u}_{j, l}\right)+O\left(a^{3}\right), \\
\mathcal{E}_{i}= & 2 a \epsilon_{i j k}\left(\tilde{u}_{j, l} \bar{B}_{k, l}-y_{, l} \tilde{u}_{j, l} \bar{B}_{k}\right) \\
& +2 a^{2} \epsilon_{i j k}\left(\tilde{u}_{j, l m} \bar{B}_{k, l m}-2\left(y_{, l m} \tilde{u}_{j, l}+y_{, l} \tilde{u}_{j, l m}\right) \bar{B}_{k, l}\right. \\
& \left.+\left(2 y_{, l} y_{, l m} \tilde{u}_{j, m}+\left(y_{, p} y_{, l}-y_{, p l}\right) \tilde{u}_{j, p l}\right) \bar{B}_{k}\right)+O\left(a^{3}\right) .
\end{aligned}
$$




\section{Paper II: A nonlinear structural subgrid-scale closure for compressible MHD. I. Derivation and energy dissipation properties}

As the Maxwell SGS stress is not directly affected by density variations, its closure is identical to the one from Eq. (14). Remarkably, to first order the compressibility effects on the Reynolds SGS stress are implicitly accounted for by the mass-weighted filtering itself. This is a consequence of the symmetry of the Reynolds SGS stress tensor $\left(\tau_{i j}^{\mathrm{u}}=\tau_{j i}^{\mathrm{u}}\right)$. Explicit density variations appear here only at second order and as second order logarithmic derivatives. Therefore, only very strong compressibility cannot be accounted for by the simple compressibility extension implied in Eq. (17). In contrast, in the EMF closure density variations appear already at first order, and at second order they are much more extensive than for $\tau^{\mathrm{u}}$. This explains the different levels of success of the simple compressibility extension ${ }^{27}$ — terms which account for compressibility effects are missing in the EMF closure but not in the Reynolds SGS stress one.

We note that combining the recurrence relation Eq. (20) with expansions of the type of Eqs. (13) and (15) allows the construction of self-consistent closures for an SGS term of any type to any order. The SGS kinetic and magnetic energies for instance are given trivially as half the traces of the Reynolds or Maxwell SGS stress tensors, respectively. If we were to construct the SGS cross-helicity $W_{\mathrm{sgs}}=\overline{\mathbf{u} \cdot \mathbf{B}}-\tilde{\mathbf{u}} \cdot \overline{\mathbf{B}}$, e.g., to gauge the correlation between kinetic and magnetic SGS effects, its closure to first order would be given by

$$
W_{\mathrm{sgs}}=2 a\left(\tilde{u}_{i, j} \bar{B}_{i, j}-\tilde{u}_{i, j} y_{, j} \bar{B}_{i}\right)+O\left(a^{2}\right) .
$$

Retaining terms to first order in $a$ is expected to provide sufficient SGS information, as suggested by the previously reported results. ${ }^{27,32-34}$ Furthermore, the computational overhead of including such closures in an LES is minimal, as they can contain at most first order derivatives in large scale primary fields.

\section{Extension for the SGS derivatives}

Direct comparison of the outlined closures with the corresponding SGS terms based on numerical data reveals directly the probity of the method. ${ }^{2}$ However, for a posteriori application of the closures in LES simulations, a further compressible effect needs to be considered.

The simple filtering operator is a convolution and as such commutes with differentiation, however, the massweighted filtering operator does not. This is critical since the SGS stress and EMF enter the evolution equations under a gradient. For the purposes of this section, let $\hat{f}$ denote the closure of an SGS term $f$ incorporating mass-weighted filtering. Then propagating the commutator between massweighted filtering and differentiation through the closure calculations above yields the following additional contributions to the momentum and induction equations:

$$
\begin{aligned}
& \widehat{\partial_{i} \tau_{i j}^{\mathrm{u}}}-\partial_{i} \widehat{\tau}_{i j}^{\mathrm{u}}=2 a \bar{\rho}\left(\tilde{u}_{i} \tilde{u}_{j, l}+\tilde{u}_{j} \tilde{u}_{i, l}\right) y_{, i l}, \\
& \quad(\nabla \widehat{\times \mathcal{E}}-\nabla \times \hat{\mathcal{E}})_{i}=2 a \epsilon_{i j k} \epsilon_{k l m} \tilde{u}_{l, p} \bar{B}_{m} y_{j p} .
\end{aligned}
$$

These expressions show the difference between applying the closure procedure to the derivatives of the SGS terms and taking derivatives of the respective closures. The additional corrections are expected to be important primarily for very strong density variations, as they contain second derivatives in the logarithmic density. This can be also seen by comparing the expressions above with the ones obtained by differentiating Eq. (16). Furthermore, they are of leading order (in $a$ ) for the derivatives of both SGS terms and these are precisely the quantities which enter the LES evolution equations and affect the large scale dynamics.

Combining the two compressibility effects leads to significant cancellation of the first order terms in the EMF closure with a final result given by

$$
(\nabla \widehat{\times \mathcal{E}})_{i}=2 a \epsilon_{i j k} \epsilon_{k l m}\left(\left(\tilde{u}_{l} \bar{B}_{m}\right)_{, j}-\left(\tilde{u}_{l, p} \bar{B}_{m}\right)_{, j} y_{, p}\right) .
$$

For the Reynolds SGS stress, the final closure can be given as

$$
\widehat{\partial_{i} \tau_{i j}^{\mathrm{u}}}=2 a\left(\bar{\rho} \tilde{u}_{i, k} \tilde{u}_{j, k}\right)_{, i}+2 a \bar{\rho}\left(\tilde{u}_{i} \tilde{u}_{j, l}+\tilde{u}_{j} \tilde{u}_{i, l}\right) y_{, i l} .
$$

Once again, the SGS Maxwell stress closure is trivially derived from Eq. (14), as it does not contain any massweighted large scale fields.

The effects of the two types of compressibility corrections can be identified by different types of a priori testing. In fact, the validity of the compressible closures was tested $a$ priori against a range of data from sub- to hypersonic turbulence simulations and benchmarked against a wide range of alternative closures ${ }^{2}$ with very positive results. In particular, we investigate their performance with respect to the resolved energy and cross-helicity dynamics (cf. Eqs. (6) and (7)). The primary compressible closures Eqs. (21) and (22) are validated by considering their effect on the spatially local (in the Eulerian sense) dynamics, i.e., in terms of the form $\left(\tau^{\mathrm{u}}\right.$. $\nabla) \cdot \tilde{u}$ and $\mathcal{E} \cdot \nabla \times \overline{\mathbf{B}}$. These terms are usually identified with contributions to the resolved energy or cross-helicity cascades. The impact of these closures on the overall resolved energy or cross-helicity dynamics, e.g., $\tilde{\mathbf{u}} \cdot\left(\nabla \cdot \tau^{\mathrm{u}}\right)$ and $\overline{\mathbf{B}} \cdot \nabla \times \mathcal{E}$, is also tested. While the impact of the differentiation commutators Eq. (24) is best tested directly in $a$ posteriori application, by comparing the results of the local and non-local a priori tests, we give an indication of the parameter regime where these extensions can be important.

\section{SCOPE OF APPLICABILITY}

The closure described above has been derived without any strong assumptions about the flow or the magnetic field. Thus, their application is not limited to turbulence simulations but can be applied in principle to any MHD simulation in which the small scales are not sufficiently well-resolved. Nevertheless, several limitations need to be kept in mind.

First, we have implicitly assumed that the filter kernel is homogeneous and isotropic and has a constant filter scale. This translates to numerical schemes with a regular grid. Furthermore, no boundary terms have been taken into account, which is consistent with periodic domains. Extensions of SGS closures to non-regular grids and non-periodic conditions have been studied in incompressible hydrodynamics. ${ }^{17}$ However, 


\section{Paper II: A nonlinear structural subgrid-scale closure for compressible MHD. I. Derivation and energy dissipation properties}

their application to the current closure is beyond the scope of this article.

Second, the described closures are derived from the analytical form of a filter kernel. As the effective kernel of an LES for a particular numerical scheme is a combination of various discretizations, e.g., grid spacing, time-stepping, differential approximations, quadrature, flux limiting, divergence cleaning (for the magnetic field), shock capturing, etc., its exact analytical form is rarely available. Additional errors stem from the truncation of the infinite series Eqs. (13) and (18), i.e., higher order closures are in principle more accurate. Depending on the convergence rate of the expansions for a particular filter, this error may also need to be considered. Conversely, due to the nonlinear combination of gradient fields, higher order closures are more prone to numerical instabilities. ${ }^{39,50}$

Finally, in LES applications, the SGS terms are based upon information contained in resolved fields, which resides above the Nyquist scale, i.e., the grid resolution. This can be represented by decomposing the effective filter kernel into a spectral kernel at the Nyquist scale and a remainder. The spectral kernel renders the inverse transfer function of the effective filter ill-defined. In order to circumvent this, a twostep procedure can be applied. First, the derivation above should be applied to the component of the effective filtering operator with a formally well-defined inverse. The spectral filter can then be applied to the resulting equations.

To allow for the mentioned inaccuracies and numerical instabilities, additional renormalization may be applied to the final closures. Parametric renormalization may also be applied to the results of a closure for a well-behaved filter, as outlined above, in order to boost its dissipative effect or render it suitable for a selection of numerical schemes. The renormalization can come in the form of constant coefficients or variable fields. Both practices are common in LES. Most canonical SGS closures include a constant coefficient whose value is calibrated dynamically or against experimental data. Allowing for distinct coefficients for the different additive terms in the proposed closures and calibrating them against a particular dataset may be used as a guide for the relative importance of the different terms in the respective flow. With respect to spatially varying modulation, the SGS energy, for instance, can be used to renormalize the strength of the SGS effects in a hydrodynamic LES with a related closure. ${ }^{51,52}$ This technique naturally requires an additional closure for the SGS energy - a common situation in hydrodynamics, ${ }^{18,30,36,51,53-55}$ where different closures are frequently combined in order to alleviate their respective shortcomings. Both types of renormalization outlined above are applied and a priori tested ${ }^{2}$ for the proposed closures, however, it is found that neither is particularly necessary nor beneficial.

\section{ENERGY AND CROSS-HELICITY DISSIPATION PROPERTIES}

One of the main functions of SGS closures is to correct for the transfer of energy across the resolution scale. Therefore, we proceed with an analysis of the dissipation properties of the proposed closures. In particular, we consider the local dissipation of the resolved kinetic energy, magnetic energy and cross-helicity given, respectively, by

$$
\Sigma^{\mathrm{u}}=-\tau_{i j} \tilde{\mathcal{S}}_{i j}, \quad \Sigma^{\mathrm{b}}=-\mathcal{E} \cdot \overline{\mathbf{J}},
$$

and

$$
\Sigma^{W}=-\frac{\tau_{i j}}{\bar{\rho}}\left(\overline{\mathcal{M}}_{i j}-\bar{B}_{j} y_{, i}\right)-\mathcal{E} \cdot \tilde{\Omega}
$$

with the usual definitions of the resolved rate-of-strain $\tilde{\mathcal{S}}_{i j}=1 / 2\left(\tilde{u}_{i, j}+\tilde{u}_{j, i}\right)$, vorticity $(\tilde{\mathbf{\Omega}})_{k}=(\nabla \times \tilde{\mathbf{u}})_{k}$, current $(\overline{\mathbf{J}})_{k}=(\nabla \times \overline{\mathbf{B}})_{k}$, and magnetic rate-of-strain $\overline{\mathcal{M}}_{i j}=1 / 2$ $\left(\bar{B}_{i, j}+\bar{B}_{j, i}\right)$. The signs of the $\Sigma$ fields are chosen such that positive values correspond to a down-scale transfer, i.e., dissipation.

We consider each dissipation term in turn. The kinetic energy dissipation can be further decomposed according to Eq. (2) into $\Sigma^{\mathrm{u}}=\Sigma_{\tau^{\mathrm{u}}}^{\mathrm{u}}+\Sigma_{\tau^{\mathrm{b}}}^{\mathrm{u}}+\Sigma_{\tau^{\mathrm{b}}}^{\mathrm{u}}$. The contribution from the Reynolds SGS stress is given by $\Sigma_{\tau^{u}}^{u}=-\tau_{i j}^{\mathrm{u}} \tilde{\mathcal{S}}_{i j}$. The results here will be the same as in the hydrodynamic limit. As a basis for comparison, consider the classical incompressible eddy-viscosity (EV) family of closures, ${ }^{56}$ which take the form $\tau^{\mathrm{u}}=-\nu_{\text {turb }} \tilde{\mathcal{S}}$ with $\operatorname{Tr}(\tilde{\mathcal{S}}) \equiv 0$ for some (usually non-negative) turbulent viscosity $\nu_{\text {turb }}$. For it $\Sigma_{\tau^{u}}^{\mathrm{u}}$ takes the form

$$
\Sigma_{\mathrm{EV}}^{\mathrm{u}}=\nu_{\mathrm{turb}} \operatorname{Tr}\left(\tilde{\mathcal{S}}^{2}\right)
$$

where $\tilde{\mathcal{S}}^{n}$ represents a tensor product, e.g., $\left(\tilde{\mathcal{S}}^{2}\right)_{i j}=\tilde{\mathcal{S}}_{i k} \tilde{\mathcal{S}}_{k j}$. As $\operatorname{Tr}\left(\tilde{\mathcal{S}}^{2}\right)$ is always non-negative, this closure can transfer energy across the resolution scale only in one direction, depending on the sign of $\nu_{\text {turb }}$, e.g., from resolved to subgrid scales for $\nu_{\text {turb }}>0$. This model can provide energy backscatter only in the compressible regime via an additional (not self-consistent) closure for the SGS kinetic energy and even then only from regions where $\operatorname{Tr}(\tilde{\mathcal{S}})>0$. This can be seen to be problematic since the presence of strong energy cascades in both directions is a key characteristic of MHD turbulence, ${ }^{57,58}$ which differentiates it from the hydrodynamic case.

In contrast, the proposed closure for the Reynolds SGS stress $\tau^{\mathrm{u}}$ can be written as

$$
\tau_{i j}^{\mathrm{u}}=2 a \bar{\rho}\left(\tilde{\mathcal{S}}_{i k} \tilde{\mathcal{S}}_{j k}+\tilde{\Omega}_{i k} \tilde{\Omega}_{j k}+\tilde{\mathcal{S}}_{i k} \tilde{\Omega}_{j k}+\tilde{\Omega}_{i k} \tilde{\mathcal{S}}_{j k}\right),
$$

with vorticity tensor $\tilde{\Omega}_{i j}=-1 / 2 \epsilon_{i j k}(\tilde{\boldsymbol{\Omega}})_{k}$. Substituting this in $\sum_{\tau^{u}}^{\mathrm{u}}$ leads to

$$
\Sigma_{\tau^{\mathrm{u}}}^{\mathrm{u}}=-2 a \bar{\rho}\left(\operatorname{Tr}\left(\widetilde{\mathcal{S}}^{3}\right)+\frac{1}{4} \widetilde{\mathbf{\Omega}}^{2} \operatorname{Tr}(\widetilde{\mathcal{S}})-\frac{1}{4} \widetilde{\mathbf{\Omega}}^{\mathrm{T}} \cdot \widetilde{\mathcal{S}} \cdot \widetilde{\mathbf{\Omega}}\right) .
$$

The first term is reminiscent of the eddy-viscosity expression, as it depends only on the strain tensor. However, there are two qualitative differences stemming from the fact that this term is cubic in $\tilde{\mathcal{S}}$. First, the larger power leads to stronger sensitivity to the resolved rate-of-strain. Second, and perhaps more importantly, this term has indefinite signature, 


\section{Paper II: A nonlinear structural subgrid-scale closure for compressible MHD. I.}

Derivation and energy dissipation properties

which allows for bi-directional energy cascade. Because of it totally compressive rate-of-strain leads to dissipation while expansion leads to back-scatter of kinetic energy.

The proposed model includes a further effect, associated with the last two terms in Eq. (31), namely, vortex stretching. This is the compressible analogue of the incompressible vortex stretching effect encoded in the last term. Geometrically, the combination of the two terms represents the interaction of the vorticity vector with the strain lying in a plane orthogonal to it. As intuition suggests, if a simple vortex tube is compressed perpendicular to its axis, its radius decreases and bigger proportion of its kinetic energy is associated with smaller scales, i.e., this leads to dissipation. Conversely, stretching a vortex shifts its associated energy to larger scales and the result is back-scatter.

Next, consider the contribution of the Maxwell SGS stress to the kinetic energy flux given by $\Sigma_{\tau^{\mathrm{b}}}^{\mathrm{u}}=\tau_{i j}^{\mathrm{b}} \tilde{\mathcal{S}}_{i j}$. The proposed closure can be written as

$$
\tau_{i j}^{\mathrm{b}}=2 a\left(\overline{\mathcal{M}}_{i k} \overline{\mathcal{M}}_{j k}+\bar{J}_{i k} \bar{J}_{j k}+\overline{\mathcal{M}}_{i k} \bar{J}_{j k}+\bar{J}_{i k} \overline{\mathcal{M}}_{j k}\right),
$$

with current tensor $\bar{J}_{i j}=-1 / 2 \epsilon_{i j k}(\overline{\mathbf{J}})_{k}$. Its contribution to the kinetic energy dissipation is given by

$$
\begin{aligned}
\Sigma_{\tau^{\mathrm{b}}}^{\mathrm{u}}= & 2 a\left(\operatorname{Tr}(\overline{\mathcal{M}} \widetilde{\mathcal{S}} \overline{\mathcal{M}})+2 \operatorname{Tr}(\overline{\mathcal{M}} \widetilde{\mathcal{S} J})+\frac{1}{4} \overline{\mathbf{J}}^{2} \operatorname{Tr}(\widetilde{\mathcal{S}})\right. \\
& \left.-\frac{1}{4} \overline{\mathbf{J}}^{\mathrm{T}} \cdot \widetilde{\mathcal{S}} \cdot \overline{\mathbf{J}}\right) .
\end{aligned}
$$

This expression is similar to the contribution of the Reynolds SGS stress. Note, however, that the entire Maxwell SGS stress works in the opposite direction to the Reynolds SGS stress (because of the different overall sign). The first term represents the interaction between the magnetic and kinetic rates-of-strain. Here, compression (i.e., negative eigenvalues of $\tilde{\mathcal{S}}$ ) leads to back-scatter, while stretching leads to dissipation. Furthermore, alignment of the eigenvectors of $\tilde{\mathcal{S}}$ and $\overline{\mathcal{M}}$ maximizes the effect of this term. The second term is associated with the amplification of magnitudes of the ratesof-strain, i.e., $\operatorname{Tr}\left(\tilde{\mathcal{S}}^{2}\right)$ and $\operatorname{Tr}\left(\overline{\mathcal{M}}^{2}\right)$. It implies that the processes which enhance kinetic and magnetic shearing simultaneously dissipate kinetic energy. The last two terms are the counterpart of the vorticity terms Eq. (31) - they are associated with current deformation analogous to the vortex stretching effect. They imply that currents perpendicular to compressive flows lead to backscatter and ones perpendicular to expanding flows - to dissipation. Currents flowing along the compressive or stretching directions have no effect on the SGS energy.

The final component of the kinetic energy flux comes from the SGS magnetic pressure

$$
\Sigma_{\tau_{k k}^{\mathrm{b}}}^{\mathrm{u}}=-\frac{1}{2} \tau_{k k}^{\mathrm{b}} \operatorname{Tr}(\widetilde{\mathcal{S}})=-2 a \operatorname{Tr}(\widetilde{\mathcal{S}})\left(\frac{\operatorname{Tr}\left(\overline{\mathcal{M}}^{2}\right)}{2}+\frac{1}{4} \overline{\mathbf{J}}^{2}\right) .
$$

It reduces the Maxwell SGS stress effects associated with the overall dilatation rate. It introduces purely compressible effects, as in the incompressible limit $\operatorname{Tr}(\tilde{\mathcal{S}})=0$. The isotropic current component $\left(\propto \operatorname{Tr}(\tilde{\mathcal{S}}) \overline{\mathbf{J}}^{2}\right)$ cancels exactly the contribution from $\Sigma_{\tau^{b}}^{\mathrm{u}}$. This re-introduces the possibility of dissipation due to compression along the current direction and emphasizes the importance of providing a closure for the total SGS pressure. Moreover, it enhances the closure's overall sensitivity to the relative orientation of the current and the kinetic rate of strain. The magnetic shear term is associated with the growth of $\operatorname{Tr}\left(\overline{\mathcal{M}}^{2}\right)$ due to overall compression.

Finally, consider the transfer of magnetic energy across the filter scale. The analytic form of $\Sigma^{\mathrm{b}}$ shows that there is backscatter or dynamo-like effect, when the electromotive force is aligned with the large-scale currents and dissipation into unresolved energy in case of anti-alignment. Decomposing the proposed closure into symmetric and anti-symmetric gradients of the resolved fields and substituting into the expression for $\Sigma^{\mathrm{b}}$, leads to the following expression:

$$
\begin{aligned}
\Sigma^{\mathrm{b}}= & 2 a\left(2 \operatorname{Tr}(\overline{\mathcal{M}} \widetilde{\mathcal{S}} \bar{J})+\frac{1}{2} \overline{\mathbf{J}}^{\mathrm{T}} \cdot \widetilde{\mathcal{S}} \cdot \overline{\mathbf{J}}-\frac{1}{2} \overline{\mathbf{J}}^{2} \operatorname{Tr}(\widetilde{\mathcal{S}})\right. \\
& -\frac{1}{2} \widetilde{\boldsymbol{\Omega}}^{\mathrm{T}} \cdot \overline{\mathcal{M}} \cdot \overline{\mathbf{J}}+(\overline{\mathbf{B}} \times \overline{\mathbf{J}})^{\mathrm{T}} \cdot \widetilde{\mathcal{S}} \cdot \nabla y \\
& \left.+\frac{1}{2}(\widetilde{\boldsymbol{\Omega}} \cdot \overline{\mathbf{B}})(\overline{\mathbf{J}} \cdot \nabla y)-\frac{1}{2}(\widetilde{\mathbf{\Omega}} \cdot \overline{\mathbf{J}})(\overline{\mathbf{B}} \cdot \nabla y)\right) .
\end{aligned}
$$

Due to the nonlinear coupling between kinetic and magnetic structures in this closure, these terms involve a large plethora of effects.

Here, like in the kinetic energy case, the relative alignment of the resolved gradients, i.e., the local inhomogeneity and anisotropy, plays a vital role in determining the magnetic energy flux. The first four terms are associated with evolution of the total current $\overline{\mathbf{J}}^{2}$. The first, shearing term is already familiar from Eq. (33) and has the same effect on the magnetic energy as on the kinetic one. The next two terms can be identified as anomalous (anisotropic) resistivity. They are also found in Eq. (33), but with opposite signs and half the amplitude. This identifies an SGS channel for transfer between resolved kinetic and magnetic energy, i.e., half of the dissipated resolved magnetic energy is backscattered into resolved kinetic energy and vice versa, kinetic energy dissipation leads to enhanced turbulence, which in turn causes a dynamo-like increase of resolved magnetic energy. The fourth term is specific to the magnetic energy budget. It is also associated with the enstrophy evolution due to the Lorentz force and connects the relative orientation of vorticity and current with the principal axes of $\overline{\mathcal{M}}$. For instance, along a magnetically compressive direction, it leads to dissipation, if the vorticity and the current are parallel, and backscatter, if they are anti-parallel.

All considerations made so far apply equally to the simple and primary compressible extensions, as well as in the incompressible limit (allowing for $\operatorname{Tr}(\tilde{\mathcal{S}})=0$ ). The final three terms of the magnetic energy dissipation Eq. (35) contain the explicit effect of the primary compressible extension. They have a strong impact primarily in regions of very strong density gradients, e.g., the neighborhood of shocks, due to the logarithmic density derivative. Formally, they are also strongly anisotropic and can be seen to be related to 


\section{Paper II: A nonlinear structural subgrid-scale closure for compressible MHD. I. Derivation and energy dissipation properties}

dynamo-like effects. For instance, $\overline{\mathbf{B}} \times \overline{\mathbf{J}}$ is the complement of the current helicity $\overline{\mathbf{B}} \cdot \overline{\mathbf{J}}$, which can be associated with the $\alpha$-dynamo, while $\tilde{\boldsymbol{\Omega}} \cdot \overline{\mathbf{J}}$ is related to the cross-helicity dynamo. ${ }^{29}$

The effect of the primary compressible extension becomes more evident when considering the SGS effects on the cross-helicity evolution. For completeness, we give the exact expressions for the local contributions of the total SGS Maxwell stress $\Sigma_{\tau_{\mathrm{tot}}^{\mathrm{b}}}^{W}=\Sigma_{\tau^{\mathrm{b}}}^{W}+\Sigma_{\tau_{k k}^{\mathrm{b}}}^{W}$, the SGS Reynolds stress $\Sigma_{\tau^{u}}^{W}$, and the EMF $\Sigma_{\mathcal{E}}^{W}$, defined analogously to their energy counterparts, to the resolved cross-helicity

$$
\begin{aligned}
& \Sigma_{\tau_{\mathrm{tot}}^{\mathrm{b}}}^{W}=-\frac{2 a}{\bar{\rho}}\left(\left(\overline{\mathbf{B}}^{\mathrm{T}} \cdot \overline{\mathcal{M}}^{2} \cdot \nabla y\right)-\operatorname{Tr}\left(\overline{\mathcal{M}}^{3}\right)\right. \\
& -\frac{1}{2} \operatorname{Tr}\left(\overline{\mathcal{M}}^{2}\right)(\overline{\mathbf{B}} \cdot \nabla y)+\overline{\mathbf{J}}^{\mathrm{T}} \cdot \overline{\mathcal{M}} \cdot \overline{\mathbf{J}} \\
& -\left(\overline{\mathbf{B}}^{\mathrm{T}} \cdot \overline{\mathcal{M}}\right) \cdot(\overline{\mathbf{J}} \times \nabla y)-(\overline{\mathbf{J}} \times \overline{\mathbf{B}})^{\mathrm{T}} \cdot(\overline{\mathcal{M}} \cdot \nabla y) \\
& -(\overline{\mathbf{J}} \cdot \overline{\mathbf{B}}) \overline{\mathbf{J}} \cdot \nabla y), \\
& \Sigma_{\tau^{u}}^{W}=2 a\left(-2 \operatorname{Tr}(\widetilde{\mathcal{S}} \overline{\mathcal{M}} \tilde{\mathbf{\Omega}})-\frac{1}{4}(\widetilde{\mathbf{\Omega}} \cdot \overline{\mathbf{B}})(\widetilde{\mathbf{\Omega}} \cdot \nabla y)\right. \\
& +\frac{1}{4} \widetilde{\mathbf{\Omega}}^{\mathrm{T}} \cdot \overline{\mathcal{M}} \cdot \widetilde{\mathbf{\Omega}}+\frac{1}{4} \widetilde{\mathbf{\Omega}}^{2}(\overline{\mathbf{B}} \cdot \nabla y) \\
& +\frac{1}{2}(\overline{\mathbf{B}} \times \widetilde{\mathbf{\Omega}})^{\mathrm{T}} \cdot(\widetilde{\mathcal{S}} \cdot \nabla y)-\operatorname{Tr}(\widetilde{\mathcal{S}} \overline{\mathcal{M}} \widetilde{\mathcal{S}}) \\
& \left.-\frac{1}{2}\left(\overline{\mathbf{B}}^{\mathrm{T}} \cdot \widetilde{\mathcal{S}}\right) \cdot(\widetilde{\mathbf{\Omega}} \times \nabla y)+\overline{\mathbf{B}}^{\mathrm{T}} \cdot \widetilde{\mathcal{S}}^{2} \cdot \nabla y\right), \\
& \Sigma_{\mathcal{E}}^{W}=2 a\left(2 \operatorname{Tr}(\widetilde{\mathcal{S}} \overline{\mathcal{M}} \tilde{\Omega})+\frac{1}{2}(\widetilde{\boldsymbol{\Omega}} \cdot \overline{\mathbf{B}})(\widetilde{\boldsymbol{\Omega}} \cdot \nabla y)\right. \\
& -\frac{1}{2} \widetilde{\boldsymbol{\Omega}}^{\mathrm{T}} \cdot \overline{\mathcal{M}} \cdot \widetilde{\boldsymbol{\Omega}}-\frac{1}{2} \widetilde{\boldsymbol{\Omega}}^{2}(\overline{\mathbf{B}} \cdot \nabla y) \\
& -(\overline{\mathbf{B}} \times \widetilde{\boldsymbol{\Omega}})^{\mathrm{T}} \cdot(\widetilde{\mathcal{S}} \cdot \nabla y)+\frac{1}{2} \widetilde{\boldsymbol{\Omega}}^{\mathrm{T}} \cdot \widetilde{\mathcal{S}} \cdot \overline{\mathbf{J}} \\
& \left.-\frac{1}{2}(\overline{\mathbf{J}} \cdot \widetilde{\mathbf{\Omega}}) \operatorname{Tr}(\widetilde{\mathcal{S}})\right) \text {. }
\end{aligned}
$$

While these expressions contain a large variety of terms, the key point is that there is a strong interplay between Reynolds SGS stress and the EMF contributions, i.e., the terms in $\Sigma_{\tau^{u}}^{W}$ and $\Sigma_{\mathcal{E}}^{W}$. For instance, the cancellation of the $\operatorname{Tr}\left(\tilde{\mathcal{S}} \overline{\mathcal{M}} \tilde{\Omega}^{\tau^{u}}\right)$ term points to an interaction between the resolved and turbulent fields which preserves the large scale topology characterized by $W$.

Another example is given by the $\nabla y$-terms in $\Sigma_{\tau^{u}}^{W}$ and $\Sigma_{\mathcal{E}}^{W}$. In $\Sigma_{\tau^{u}}^{W}$ they come from the intrinsic compressibility effect described by $\tau_{i j}^{\mathrm{u}} \bar{B}_{j} y_{, i} / \bar{\rho}$, i.e., the interaction between velocity fluctuations, density gradients, and a large scale magnetic field. The corresponding $\nabla y$-terms in $\Sigma_{\mathcal{E}}^{W}$ are specific to the primary compressible extension. The analogous form of the two sets of terms shows that the primary extension naturally restores the symmetry between kinetic and magnetic turbulent contributions to the effects of compressibility on $W_{\text {res }}$. As the resolved cross-helicity plays a role in the non-local transfer between kinetic and magnetic energies and affects the rate of energy decay, it is clearly important to treat it with as much care as the resolved energy itself.

\section{CONCLUSION}

The high computational cost of 3-dimensional direct numerical MHD simulations poses severe limitations to our understanding of astrophysical and terrestrial phenomena involving strongly turbulent magnetized fluids. Large-eddy simulations can alleviate this issue by explicitly considering the effects of limited resolution. In this work, we presented the derivation and properties of a nonlinear structural closure of the compressible MHD LES equations. It is based on a series expansion ${ }^{31}$ of the finite resolution operator, a convolution with a low-pass filter kernel, and careful consideration of the impact of the operator on the compressible dynamics. As the derivation needs no assumptions on the nature of the flow, the closures can be applied to a wide variety of MHD problems, as long as they can be described on a regular grid under periodic boundary conditions. In particular, no assumptions were invoked on the level of compressibility, on the structure, dynamics, or even the presence of turbulence and magnetic fields. Thus, the closures are suitable for both statistically stationary and developing disordered velocity and magnetic field configurations, from the sub- to the hyper-sonic and -Alfvenic regime. Only an isothermal equation of state was considered. However, the formalism can be extended to incorporate thermal variations, as well as additional evolution equations, e.g., for the SGS energy or for passive scalar transport.

Although the closures for the MHD SGS terms are derived self-consistently, the information gap below the Nyquist frequency as well as the complicated nature of realistic LES filters leaves room for additional re-normalization or re-calibration of the proposed closures and for combinations with additional closures. In fact a simple renormalized version of the closure has already been validated ${ }^{27}$ in a priori comparison. Here, through a self-consistent derivation of the compressibility effects due to a mass-weighted filter, some of the results of this comparison are clarified. An analysis of the energy dissipation properties of the simple compressible closure demonstrates that it can already accommodate sophisticated energy transfers between resolved and unresolved kinetic and magnetic energy budgets. It emphasizes the dependence of the transfer on local geometry, e.g., anisotropy, and topology, e.g., the interplay between vortical and shearing magnetic and kinetic structures of different types. Furthermore, it allows for imperfect transfer between the resolved kinetic and magnetic energy mediated by the subgrid scales. The additional effects of the self-consistent, primary closure are revealed through the resolved magnetic energy dissipation, where it plays a role in regions of strong compressibility. Moreover, it restores the symmetry between kinetic and magnetic contributions to the cross-helicity dissipation and thus plays a vital role in the evolution of the large-scale fields' topology. Thus presented, the closure is ready to be bench-marked against currently used compressible MHD closures and to have its properties validated against numerical and experimental turbulence data. The results of such a comparison with a wide selection of available SGS closures against a suite of simulation data of homogeneous and isotropic turbulence ranging from the sub- to the hyper-sonic regime are presented in Paper II. ${ }^{2}$ 


\section{Paper II: A nonlinear structural subgrid-scale closure for compressible MHD. I. Derivation and energy dissipation properties}

\section{ACKNOWLEDGMENTS}

P.G. acknowledges financial support by the International Max Planck Research School for Solar System Science at the University of Göttingen. D.V. acknowledges research funding by the Deutsche Forschungsgemeinschaft (DFG) under Grant No. SFB 963/1, project A15 and the Max Planck Institute for Dynamics and Self-Organization. D.R.G.S. thanks for funding through Fondecyt regular (project code 1161247) and through the "Concurso Proyectos Internacionales de Investigación, Convocatoria 2015" (project code PII20150171). This project was supported by the North-German Supercomputing Alliance under Grant No. nip00037.

${ }^{1} \mathrm{~W} . \mathrm{K}$. Yeo, "Conjunctive filtering procedures in surface water flow and transport," in Large Eddy Simulation of Complex Engineering and Geophysical Flows, edited by B. Galperin and S. A. Orszag (CUP, 1993).

${ }^{2}$ P. Grete, D. G. Vlaykov, W. Schmidt, and D. R. G. Schleicher, Phys. Plasmas 23, 062317 (2016).

${ }^{3}$ K. Subramanian, A. Shukurov, and N. E. L. Haugen, Mon. Not. R. Astron Soc. 366, 1437 (2006); e-print arXiv:0505144 [astro-ph]

${ }^{4}$ J. Cho, Astrophys. J. 797, 133 (2014); e-print arXiv:1410.1893.

${ }^{5}$ M. A. Latif, D. R. G. Schleicher, W. Schmidt, and J. Niemeyer, Mon. Not. R. Astron. Soc. 432, 668 (2013).

${ }^{6}$ M.-M. Mac Low and R. S. Klessen, Rev. Mod. Phys. 76, 125 (2004); eprint arXiv:0301093 [astro-ph].

${ }^{7}$ D. J. Price and M. R. Bate, Mon. Not. R. Astron. Soc. 385, 1820 (2008); eprint arXiv:0801.3293.

${ }^{8}$ P. Hennebelle and R. Teyssier, Astron. Astrophys. 477, 25 (2008); e-print arXiv:0709.2887.

${ }^{9}$ S. A. Balbus and J. F. Hawley, Rev. Mod. Phys. 70, 1 (1998).

${ }^{10}$ D. Biskamp, Magnetohydrodynamic Turbulence (CUP, 2003), p. 310

${ }^{11}$ R. E. Pudritz, R. Ouyed, C. Fendt, and A. Brandenburg, Protostars and Planets V, edited by B. Reipurth, D. Jewitt, and K. Keil (The University of Arizona Press Tucson in collaboration with Lunar and planetary Institute, Houston, 2007), p. 277; e-print arXiv:astro-ph/0603592.

${ }^{12}$ E. Priest, Magnetohydrodynamics of the Sun (CUP, 2014).

${ }^{13}$ Y. Zhou, W. Matthaeus, and P. Dmitruk, Rev. Mod. Phys. 76, 1015 (2004).

${ }^{14}$ A. Mangeney, R. Grappin, and M. Velli, in Advances in Solar System Magnetohydrodynamics, edited by E. R. Priest and A. W. Hood (CUP 1991), p. 327.

${ }^{15}$ A. A. Chernyshov, K. V. Karelsky, and A. S. Petrosyan, Phys. Plasmas 17, 102307 (2010).

${ }^{16}$ R. Bruno and V. Carbone, Living Rev. Sol. Phys. 10, 1 (2013).

${ }^{17}$ P. Sagaut, Large Eddy Simulation for Incompressible Flows (Springer, Berlin, Heidelberg, 2006)

${ }^{18}$ E. Garnier, N. Adams, and P. Sagaut, Large Eddy Simulation for Compressible Flows, Scientific Computation (Springer Netherlands, 2009).

${ }^{19}$ M. Miesch, W. Matthaeus, A. Brandenburg, A. Petrosyan, A. Pouquet, C. Cambon, F. Jenko, D. Uzdensky, J. Stone, S. Tobias, J. Toomre, and M. Velli, Space Sci. Rev. 194, 97 (2015); e-print arXiv:1505.01808 [astroph.SR].

${ }^{20}$ W. Schmidt, Living Rev. Comput. Astrophys. 1 (2015).

${ }^{21}$ A. A. Chernyshov, K. V. Karelsky, and A. S. Petrosyan, Phys. - Usp. 57, 421 (2014).
${ }^{22}$ A. Favre, Phys. Fluids 26, 2851 (1983).

${ }^{23}$ D. G. Vlaykov, Sub-grid Scale Modelling of Compressible Magnetohydrodynamic Turbulence: Derivation and A Priori Analysis, Ph.D. thesis, Georg-August-Universität Göttingen, 2015.

${ }^{24}$ W.-C. Müller and D. Carati, Comput. Phys. Commun. 147, 544 (2002).

${ }^{25}$ W.-C. Müller and D. Carati, Phys. Plasmas 9, 824 (2002).

${ }^{26}$ J. P. Graham, P. D. Mininni, and A. Pouquet, Phys. Rev. E 80, 016313 (2009); e-print arXiv:0806.2054.

${ }^{27}$ P. Grete, D. G. Vlaykov, W. Schmidt, D. R. G. Schleicher, and C. Federrath, New J. Phys. 17, 023070 (2015).

${ }^{28}$ F. Widmer, J. Büchner, and N. Yokoi, Phys. Plasmas 23, 042311 (2016).

${ }^{29}$ N. Yokoi, Geophys. Astrophys. Fluid Dyn. 107, 114 (2013).

${ }^{30}$ J. Bardina, J. H. Ferziger, and W. C. Reynolds, "Improved turbulence models based on large eddy simulation of homogeneous, incompressible turbulent flows," Technical Report No. TF-19, Stanford University, May 1983.

${ }^{31}$ W. K. Yeo, A Generalized High Pass/Low Pass Averaging Procedure for Deriving and Solving Turbulent Flow Equations, Ph.D. thesis, Ohio State University, 1987.

${ }^{32}$ G. Balarac, H. Pitsch, and V. Raman, Phys. Fluids 20, 091701 (2008).

${ }^{33}$ Y. Fabre and G. Balarac, Phys. Fluids 23, 115103 (2011).

${ }^{34}$ G. Balarac, J. Le Sommer, X. Meunier, and A. Vollant, Phys. Fluids 25, 075107 (2013).

${ }^{35}$ C. D. Pruett, J. S. Sochacki, and N. A. Adams, Phys. Fluids 13, 2578 (2001).

${ }^{36}$ D. Carati, G. S. Winckelmans, and H. Jeanmart, J. Fluid Mech. 441, 119 (2001).

${ }^{37}$ R. A. Clark, J. H. Ferziger, and W. C. Reynolds, J. Fluid Mech. 91, 1 (1979).

${ }^{38}$ A. Leonard, Adv. Geophys. 18A, 237 (1974).

${ }^{39}$ B. Vreman, B. Geurts, and H. Kuerten, Theor. Comput. Fluid Dyn. 8, 309 (1996).

${ }^{40}$ M. D. Love, J. Fluid Mech. 100, 87 (1980).

${ }^{41}$ P. H. van Cittert, Z. Phys. 69, 298 (1931).

${ }^{42}$ S. Stolz and N. A. Adams, Phys. Fluids 11, 1699 (1999).

${ }^{43}$ S. Stolz, N. A. Adams, and L. Kleiser, Phys. Fluids 13, 997 (2001).

${ }^{44}$ S. Stolz, N. A. Adams, and L. Kleiser, Phys. Fluids 13, 2985 (2001).

${ }^{45}$ S. Stolz and N. A. Adams, Phys. Fluids 15, 2398 (2003).

${ }^{46}$ S. Stolz, P. Schlatter, and L. Kleiser, Phys. Fluids 17, 065103 (2005).

${ }^{47}$ S. Stolz, P. Schlatter, and L. Kleiser, AIAA J. 45, 1019 (2007).

${ }^{48}$ L. Bin and W. Songping, Chin. J. Aeronaut. 20, 495 (2007).

${ }^{49}$ G. Erlebacher, M. Y. Hussaini, C. G. Speziale, and T. A. Zang, J. Fluid Mech. 238, 155 (1992)

${ }^{50}$ B. J. Geurts, Phys. Fluids 9, 3585 (1997)

${ }^{51}$ W. Schmidt and C. Federrath, Astron. Astrophys. 528, A106 (2011).

${ }^{52}$ P. R. Woodward and D. H. Porter, Numerical Modeling of Space Plasma Flows, ASP Conference Series Vol. 359, p. 97, edited by Nikolai V. Pogorelov and Gary P. Zank (2006).

${ }^{53}$ C. G. Speziale, G. Erlebacher, T. A. Zang, and M. Y. Hussaini, Phys. Fluids 31, 940 (1988)

${ }^{54}$ W. Schmidt, J. C. Niemeyer, and W. Hillebrandt, Astron. Astrophys. 450, 265 (2006); e-print arXiv:0601499 [astro-ph].

${ }^{55}$ L. Iapichino, A. Maier, W. Schmidt, and J. C. Niemeyer, AIP Conf. Proc. 1241, 928-934 (2010); e-print arXiv:0911.2629.

${ }^{56}$ A. A. Chernyshov, K. V. Karelsky, and A. S. Petrosyan, Phys. Fluids 19, 055106 (2007).

${ }^{57}$ A. Pouquet, U. Frisch, and J. Léorat, J. Fluid Mech. 77, 321 (1976)

${ }^{58}$ W.-C. Müller, S. K. Malapaka, and A. Busse, Phys. Rev. E: Stat., Nonlinear, Soft Matter Phys. 85, 3 (2012); e-print arXiv:1201.0717. 


\section{Paper III: A nonlinear structural subgrid-scale closure for compressible MHD. II. A priori comparison on turbulence simulation data}

The paper originally appeared as Philipp Grete, Dimitar G. Vlaykov, Wolfram Schmidt, and Dominik R. G. Schleicher. A nonlinear structural subgrid-scale closure for compressible MHD. II. A priori comparison on turbulence simulation data. Physics of Plasmas, 23 (6):062317, 2016. doi: 10.1063/1.4954304.

The research was jointly designed and planned by all authors. PG ran the Enzo simulations, conducted the analysis in sections IV.B., IV.C. and IV.E. and extended the initial analysis by Vlaykov (2015) in sections IV.A. and IV.D. The text was written by PG in constant exchange with the coauthors. 


\title{
A nonlinear structural subgrid-scale closure for compressible MHD. II. A priori comparison on turbulence simulation data
}

\author{
Philipp Grete, ${ }^{1,2, a)}$ Dimitar G. Vlaykov, ${ }^{2,3}$ Wolfram Schmidt, ${ }^{4}$ and Dominik R. G. Schleicher ${ }^{5}$ \\ ${ }^{1}$ Max-Planck-Institut für Sonnensystemforschung, Justus-von-Liebig-Weg 3, D-37077 Göttingen, Germany \\ ${ }^{2}$ Institut für Astrophysik, Universität Göttingen, Friedrich-Hund-Platz 1, D-37077 Göttingen, Germany \\ ${ }^{3}$ Max-Planck-Institut für Dynamik und Selbstorganisation, Am Faßberg 17, D-37077 Göttingen, Germany \\ ${ }^{4}$ Hamburger Sternwarte, Universität Hamburg, Gojenbergsweg 112, D-21029 Hamburg, Germany \\ ${ }^{5}$ Departamento de Astronomía, Facultad Ciencias Físicas y Matemáticas, Universidad de Concepción, \\ Av. Esteban Iturra s/n Barrio Universitario, Casilla 160-C, Chile
}

(Received 5 February 2016; accepted 31 May 2016; published online 28 June 2016)

Even though compressible plasma turbulence is encountered in many astrophysical phenomena, its effect is often not well understood. Furthermore, direct numerical simulations are typically not able to reach the extreme parameters of these processes. For this reason, large-eddy simulations (LES), which only simulate large and intermediate scales directly, are employed. The smallest, unresolved scales and the interactions between small and large scales are introduced by means of a subgridscale (SGS) model. We propose and verify a new set of nonlinear SGS closures for future application as an SGS model in LES of compressible magnetohydrodynamics. We use 15 simulations (without explicit SGS model) of forced, isotropic, homogeneous turbulence with varying sonic Mach number $\mathrm{M}_{\mathrm{s}}=0.2-20$ as reference data for the most extensive a priori tests performed so far in literature. In these tests, we explicitly filter the reference data and compare the performance of the new closures against the most widely tested closures. These include eddy-viscosity and scalesimilarity type closures with different normalizations. Performance indicators are correlations with the turbulent energy and cross-helicity flux, the average SGS dissipation, the topological structure and the ability to reproduce the correct magnitude and the direction of the SGS vectors. We find that only the new nonlinear closures exhibit consistently high correlations (median value $>0.8$ ) with the data over the entire parameter space and outperform the other closures in all tests. Moreover, we show that these results are independent of resolution and chosen filter scale. Additionally, the new closures are effectively coefficient-free with a deviation of less than $20 \%$. Published by AIP Publishing. [http://dx.doi.org/10.1063/1.4954304]

\section{INTRODUCTION}

Turbulence and in particular, plasma turbulence is still one of the least understood phenomena in classical physics today. Even though there are advances in theory, many processes cannot be fully explained yet due to their strong nonlinearity. These cover many different scales and include experiments on Earth ${ }^{1}$ as well as a wide variety of processes (e.g., magnetic reconnection ${ }^{2}$ and turbulent dynamos ${ }^{3}$ ) and astrophysical phenomena such as stellar winds ${ }^{4}$ and magnetized accretion disks. ${ }^{5}$ Compressibility also plays an important role in astrophysical plasmas and increases the complexity even further.

In addition to theory, experiments, and observations, numerical simulations are a useful tool to understand turbulence. However, the level of detail is restricted by the available computing power, and realistic (physical) dynamical ranges are usually not covered. Fortunately, this problem can be improved with the help of large eddy simulations (LES). ${ }^{6,7}$ This approach simulates only the largest and intermediate scales directly. The smallest scales, which are below the resolution limit, i.e., below the grid scale, are introduced by means of a subgrid-scale (SGS) model. Formally, the

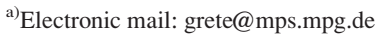

procedure involves the convolution of the primary equations with a filter kernel $G$. For a static, homogeneous, and isotropic filter, the compressible magnetohydrodynamics (MHD) equations under boundary conditions read ${ }^{8,9}$

$$
\begin{gathered}
\frac{\partial \bar{\rho}}{\partial t}+\nabla \cdot(\bar{\rho} \widetilde{\boldsymbol{u}})=0, \\
\frac{\partial \bar{\rho} \widetilde{\boldsymbol{u}}}{\partial t}+\nabla \cdot(\bar{\rho} \widetilde{\boldsymbol{u}} \otimes \widetilde{\boldsymbol{u}}-\overline{\boldsymbol{B}} \otimes \overline{\boldsymbol{B}})+\nabla\left(\bar{P}+\frac{\bar{B}^{2}}{2}\right) \\
=\nabla \cdot\left(2 \nu \bar{\rho} \widetilde{\mathcal{S}}^{*}\right)-\nabla \cdot \tau, \\
\frac{\partial \overline{\boldsymbol{B}}}{\partial t}-\nabla \times(\widetilde{\boldsymbol{u}} \times \overline{\boldsymbol{B}})+\eta \nabla^{2} \overline{\boldsymbol{B}}=\nabla \times \mathcal{E} .
\end{gathered}
$$

Filtering is denoted by $\bar{\square}$, and mass-weighted filtering ${ }^{10}$ is denoted by $\widetilde{\square}=\overline{\rho \square} / \bar{\square}$. Thus, $\bar{\rho}, \widetilde{\boldsymbol{u}}, \overline{\boldsymbol{B}}$ (incorporating $1 / \sqrt{4 \pi}$ ), and $\bar{P}$ are the filtered density, velocity, magnetic field, and thermal pressure, respectively. In the context of LES, filtered quantities are considered resolved and therefore accessible in the simulation. Non-ideal effects are included via resistivity $\eta$ and kinematic viscosity $\nu$ with traceless kinetic rate-ofstrain tensor $\widetilde{\mathcal{S}}_{i j}^{*}=1 / 2\left(\widetilde{u}_{i, j}+\widetilde{u}_{j, i}\right)-1 / 3 \delta_{i j} \widetilde{u}_{k, k}$. Here, $\square_{i, j}$ designates the $j$ th partial derivative of component $i$, a star $\square_{i j}^{*}$ indicates the traceless, deviatoric part of a tensor, and Einstein summation convention applies with the Kronecker delta $\delta_{i j}$. 


\section{Paper III: A nonlinear structural subgrid-scale closure for compressible MHD. II. A priori comparison on turbulence simulation data}

Two new terms enter Equations (2) and (3). The first term, modified from its hydrodynamical form, is the turbulent stress tensor

$$
\begin{aligned}
\tau_{i j} & =\tau_{i j}^{\mathrm{u}}-\tau_{i j}^{\mathrm{b}}+\left(\overline{B^{2}}-\bar{B}^{2}\right) \frac{\delta_{i j}}{2} \quad \text { with } \\
\tau_{i j}^{\mathrm{u}} & \equiv \bar{\rho}\left(\widetilde{u_{i} u_{j}}-\widetilde{u}_{i} \widetilde{u}_{j}\right) \quad \text { and } \quad \tau_{i j}^{\mathrm{b}} \equiv\left(\overline{B_{i} B_{j}}-\bar{B}_{i} \bar{B}_{j}\right),
\end{aligned}
$$

which consists of the turbulent (or SGS) magnetic pressure (last term in (4)), the SGS Reynolds stress $\tau_{i j}^{\mathrm{u}}$, and the SGS Maxwell stress $\tau_{i j}^{\mathrm{b}}$. The second term is the turbulent electromotive force (EMF)

$$
\mathcal{E}=\overline{\boldsymbol{u} \times \boldsymbol{B}}-\widetilde{\boldsymbol{u}} \times \overline{\boldsymbol{B}}
$$

in the induction equation. Both terms are a priori unknown as only filtered primary quantities are accessible in LES (e.g., $\widetilde{\boldsymbol{u}}$ ) but no mixed terms (e.g., $\widetilde{u_{i} u_{j}}$ ). Moreover, the total filtered energy density

$$
\bar{E}=\underbrace{\frac{1}{2} \bar{\rho} \widetilde{u}^{2}+\frac{1}{2} \bar{B}^{2}}_{\text {(resolved) }}+\underbrace{\frac{1}{2} \bar{\rho}\left(\widetilde{u^{2}}-\widetilde{u}^{2}\right)+\frac{1}{2}\left(\overline{B^{2}}-\bar{B}^{2}\right)}_{=E_{\mathrm{sgs}}^{\mathrm{u}}+E_{\mathrm{ggs}}^{\mathrm{b}} \equiv E_{\mathrm{sgg}}(\text { unresolved })}
$$

contains unclosed terms as well, namely, the kinetic SGS energy $E_{\mathrm{sgs}}^{\mathrm{u}}$ and magnetic SGS energy $E_{\mathrm{sgs}}^{\mathrm{b}}$. These terms are given by the isotropic parts of the turbulent stress tensors

$$
\frac{1}{2} \tau_{k k}^{\square}=E_{\mathrm{sgs}}^{\square} .
$$

Similarly, the filtering procedure applies to other quantities such as the cross-helicity $W-$ a measure of the alignment between velocity and magnetic field. The resulting SGS cross-helicity $W_{\text {sgs }}$ is given by

$$
W_{\mathrm{sgs}}=\overline{\boldsymbol{u} \cdot \boldsymbol{B}}-\widetilde{\boldsymbol{u}} \cdot \overline{\boldsymbol{B}}
$$

It encodes not only the alignment between unresolved fields but also between resolved and unresolved ones.

On the one hand, there has been a lot of research in the realm of (incompressible) hydrodynamics ${ }^{11}$ with successful applications to atmospheric boundary layers ${ }^{12}$ and turbulent mixing, ${ }^{13,14}$ as well as astrophysical application ${ }^{6}$ in different subjects such as isolated disc galaxies ${ }^{15}$ or the formation of supermassive black holes. ${ }^{16}$ On the other hand, results for MHD are still scarce and limited to a posteriori application of (decaying) turbulent boxes ${ }^{17}$ in either $2 \mathrm{D},{ }^{18}$ or in the incompressible case, ${ }^{19,20}$ or by neglecting terms such as turbulent magnetic pressure. ${ }^{8}$ However, the a priori validation of these closures is still outstanding, apart from a single incompressible dataset for the EMF. ${ }^{21}$ For this reason, we here expand our first investigation of nonlinear closures ${ }^{22}$ with additional closures from the literature, and over a more extended set of parameters and test cases. We have identified several closure strategies developed in the literature and evaluate the three major ones: eddy-viscosity, which is typically purely dissipative, scale-similarity, which is based on the self-similar properties of turbulence, and deconvolution closures, which are fundamentally nonlinear based on approximate inverses of the filtering operator. All closures, including the new nonlinear closures, are briefly presented in Sec. II. A detailed derivation and formal analysis of the new closures are described in Paper $\mathrm{I}^{23}$ In Section III, we describe our test setup and the process of a priori testing for several reference quantities. The results are then illustrated in Section IV and include a wide variety of functional and structural tests. Finally, in Section V, we conclude with an overall comparison of the presented closures.

\section{CLOSURES}

The following independent terms require closures: the SGS Reynolds stress $\tau^{\mathrm{u}}$, the SGS Maxwell stress $\tau^{\mathrm{b}}$, and the electromotive force $\mathcal{E}$. In the following, we briefly present three general closure strategies (eddy-viscosity, scalesimilarity, and nonlinear) and possible variations with respect to normalization. Each closure strategy is based on a certain idea that naturally transfers to closures of all unknown terms. We identify closures by two uppercase roman letters (with normalizations in superscript), and closure expressions in formulas are denoted by a hat $\hat{\square}$.

\section{A. Eddy-dissipation closures}

The eddy-dissipation family is the most well-established type of closure originating from the Smagorinsky eddy-viscosity $^{24}$ going back several decades. In general, the modeled effects are purely dissipative in nature and resemble existing terms, e.g., the Reynolds stress (10) has the same functional form as the microscopic dissipation in the momentum equation, cf. the right hand side of (2). The same is true for the EMF (12) and Ohmic dissipation in the induction equation. An eddy-diffusivity based closure for the Maxwell stress has been proposed ${ }^{17}$ analogous to eddy-viscosity. The resulting closures are

$$
\begin{array}{ll}
\text { EV }: & \hat{\tau}_{i j}^{\mathrm{u} *}=-2 \bar{\rho} \nu^{\mathrm{u}} \widetilde{\mathcal{S}}_{i j}^{*}, \\
\text { ED }: & \hat{\tau}_{i j}^{\mathrm{b} *}=-2 \nu^{\mathrm{b}} \overline{\mathcal{M}}_{i j}, \\
\text { ER }: & \hat{\mathcal{E}}=-\eta_{t} \overline{\mathbf{J}},
\end{array}
$$

with eddy-viscosity $(\mathrm{EV}) \nu^{\mathrm{u}}$, diffusivity (ED) $\nu^{\mathrm{b}}$, resistivity (ER) $\eta_{t}$, and resolved current $\overline{\mathbf{J}}=\nabla \times \overline{\boldsymbol{B}}$. The kinetic rateof-strain tensor $\widetilde{\mathcal{S}}_{i j}^{*}$ and magnetic rate-of-strain tensor $\overline{\mathcal{M}}_{i j}=$ $1 / 2\left(\bar{B}_{i, j}+\bar{B}_{j, i}\right)$ are by construction deviatoric and so are the closures (10) and (11). The remaining isotropic parts are closed by means of SGS energy closures

$$
\hat{E}_{\mathrm{sgs}}^{\mathrm{b}, \mathcal{M}}=C_{1} \Delta^{2}|\overline{\mathcal{M}}|^{2} \quad \text { and } \quad \hat{E}_{\mathrm{sgs}}^{\mathrm{u}, \mathcal{S}^{*}}=C_{2} \Delta^{2} \bar{\rho}\left|\widetilde{\mathcal{S}}^{*}\right|^{2},
$$

which can be derived from (10) and (11) building upon the realizability of $\hat{\tau}_{i j}^{\mathrm{u}}$ and $\hat{\tau}_{i j}^{\mathrm{b}}$ for a positive filter kernel. ${ }^{22,25}$ The free coefficients $C_{\square}$ appear independently in every closure (including all following ones) and are typically dimensionless. One goal of a priori testing is the determination of the coefficient values as described in Subsection III B.

In addition to the realizability ansatz, the isotropic parts can be closed under the assumption of local equilibrium 
between production and dissipation in the SGS energy evolution equations ${ }^{9}$ resulting in

$$
\hat{E}_{\mathrm{sgs}}^{\mathrm{b}, J}=C_{3} \Delta^{2}|\bar{J}|^{2} \quad \text { and } \quad \hat{E}_{\mathrm{sgs}}^{\mathrm{u}, \mathcal{S}}=C_{4} \Delta^{2} \bar{\rho}|\widetilde{\mathcal{S}}|^{2} .
$$

Furthermore, several normalizations (or scalings) have been developed to control the strength of the deviatoric closures based on different arguments. In this paper, we test the most often used ones, i.e., constant scaling, scaling by SGS energy, and scaling by the interaction between the velocity and the magnetic field. Constant scaling is given by

$$
\begin{array}{ll}
\mathrm{EV}^{\text {const }}: & \nu^{\mathrm{u}}=C_{5} \Delta^{4 / 3}, \\
\mathrm{ED}^{\text {const }}: & \nu^{\mathrm{b}}=C_{6} \Delta^{4 / 3}, \\
\mathrm{ER}^{\text {const }}: & \eta_{t}=C_{7} \Delta^{4 / 3},
\end{array}
$$

motivated by dimensional analysis under Kolmogorov scaling. ${ }^{26}$ These closures neglect any local variability of the eddy-viscosity, diffusivity, and resistivity. In contrast to this, SGS energies, as a local measure of unresolved turbulence, can be used as a proxy to obtain spatially varying closures

$$
\begin{array}{ll}
\mathrm{EV}^{E}: & \nu^{\mathrm{u}}=C_{13} \Delta \sqrt{E_{\mathrm{sgs}}^{\mathrm{u}} / \bar{\rho}}, \\
\mathrm{ED}^{E}: & \nu^{\mathrm{b}}=C_{14} \Delta \sqrt{E_{\mathrm{sgs}}^{\mathrm{b}}}, \\
\mathrm{ER}^{E}: & \eta_{t}=C_{15} \Delta \sqrt{\left(E_{\mathrm{sgs}}^{\mathrm{u}}+E_{\mathrm{sgs}}^{\mathrm{b}}\right) / \bar{\rho}} .
\end{array}
$$

However, the exact values for the energies $E_{\mathrm{sgs}}^{\mathrm{u}}$ and $E_{\mathrm{sgs}}^{\mathrm{b}}(7)$ are unknown. Thus, the energy closure expressions (13) can be used to formulate complete closures ${ }^{18}$ based only on known fields

$$
\begin{aligned}
& \mathrm{EV}^{\mathcal{S}^{*}}: \quad \nu^{\mathrm{u}}=C_{16} \Delta \sqrt{\hat{E}_{\mathrm{sgs}}^{\mathrm{u}, \mathcal{S}^{*}} / \bar{\rho}}, \\
& \mathrm{ED}^{\mathcal{M}}: \quad \nu^{\mathrm{b}}=C_{17} \Delta \sqrt{\hat{E}_{\mathrm{sgs}}^{\mathrm{b}, \mathcal{M}}}, \\
& \mathrm{ER}^{\mathcal{S}+\mathcal{M}}: \quad \eta_{t}=C_{18} \Delta \sqrt{\left(\hat{E}_{\mathrm{sgs}}^{\mathrm{u}, \mathcal{S}^{*}}+\hat{E}_{\mathrm{sgs}}^{\mathrm{b}, \mathcal{M}}\right) / \bar{\rho}} .
\end{aligned}
$$

Another possibility to include local variability is via the interactions between velocity and magnetic field. Here, the SGS cross-helicity (9) serves as a proxy in the closures

$$
\begin{array}{ll}
\mathrm{EV}^{W}: & \nu^{\mathrm{u}}=C_{10} \Delta \rho^{-1 / 4} \sqrt{\left|W_{\mathrm{sgs}}\right|}, \\
\mathrm{ED}^{W}: & \nu^{\mathrm{b}}=C_{11} t_{t} W_{\mathrm{sgs}}, \\
\mathrm{ER}^{W}: & \eta_{t}=C_{12} t_{t} \sqrt{\bar{\rho}} W_{\mathrm{sgs}},
\end{array}
$$

with a turbulent time scale $t_{t}=\Delta \sqrt{\bar{\rho} / E_{\mathrm{sgg}}}$. Again, an alternative formulation has been proposed ${ }^{19}$

$$
\begin{aligned}
& \mathrm{EV}^{\mathcal{S M}}: \quad \nu^{\mathrm{u}}=C_{8} \Delta^{2} \rho^{-1 / 4} \sqrt{\mid 2 \widetilde{\mathcal{S}_{i j} \overline{\mathcal{M}_{i j}} \mid}}, \\
& \mathrm{ER}^{\mathcal{S M}}: \quad \eta_{t}=C_{9} \Delta^{2} \operatorname{sgn}(\overline{\mathbf{J}} \cdot \widetilde{\mathbf{\Omega}}) \sqrt{|\overline{\mathbf{J}} \cdot \widetilde{\mathbf{\Omega}}| / \bar{\rho}^{1 / 2}},
\end{aligned}
$$

since (9) is unclosed. $\widetilde{\boldsymbol{\Omega}}=\nabla \times \widetilde{\boldsymbol{u}}$ is the resolved vorticity. The closures are motivated by assuming that the modeled cross-helicity dissipation rate is a robust proxy of transfer between the kinetic and the magnetic energy.

In addition, we include the $\alpha-\beta-\gamma$-closure ${ }^{20}$ for the electromotive force

$$
\alpha-\beta-\gamma: \quad \eta_{t}=\alpha \overline{\boldsymbol{B}}-\beta \overline{\mathbf{J}}+\gamma \widetilde{\boldsymbol{\Omega}}
$$

in our comparison which was recently applied in LES of current sheets. ${ }^{27}$ Here, $\beta$ is closed identically to $\mathrm{ER}^{E}, \gamma=$ $C_{19} t_{t} W_{\mathrm{sgs}}$ is linked to the SGS cross-helicity, and $\alpha=C_{20} t_{t} H$ is connected to the residual helicity $H=\overline{\boldsymbol{u} \cdot \boldsymbol{\Omega}}-\widetilde{\boldsymbol{u}} \cdot \widetilde{\boldsymbol{\Omega}}$ $-(\overline{\boldsymbol{B} \cdot \boldsymbol{J}}-\overline{\boldsymbol{B}} \cdot \overline{\boldsymbol{J}}) / \bar{\rho}$.

\section{B. Scale-similarity closure}

Scale-similarity (SS) closures are characterized by the assumption that the tensorial structure at the smallest resolved scales is similar to the one at the largest unresolved scales. ${ }^{28}$ This motivates the introduction of a second filter (a test filter) with a filter width equal to or larger than the original filter width. The result of the second filter operation is analogous to the result of the first filter operation, and this allows the recovery of the subgrid-scales. We use a filter with twice the original filter width, as proposed based on ex-

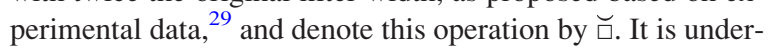
stood that mass-weighted filtering is applied to all quantities involving $\widetilde{\boldsymbol{u}}$. The resulting closures are

$$
\begin{aligned}
& \mathrm{SS}^{\mathrm{u}}: \quad \hat{\tau}_{i j}^{\mathrm{u}}=C_{21} \overline{\bar{\rho}}(\underbrace{\widetilde{\widetilde{u}_{i}}}_{\left(\widetilde{u_{i}} \widetilde{u}_{j}\right.} \widetilde{\widetilde{u}}_{j}), \\
& \mathrm{SS}^{\mathrm{b}}: \quad \hat{\tau}_{i j}^{\mathrm{b}}=C_{22}\left(\overline{\overline{B_{i}} \overline{B_{j}}}-\overline{B_{i}} \overline{B_{j}}\right), \\
& \mathrm{SS}^{\mathcal{E}}: \quad \hat{\mathcal{E}}=C_{23}(\underbrace{}_{\boldsymbol{\boldsymbol { u }} \times \overline{\boldsymbol{B}}}-\widetilde{\boldsymbol{u}} \times \overline{\boldsymbol{B}}) .
\end{aligned}
$$

It should be noted that these coefficients are introduced in order to allow for deviation from model assumptions. Nevertheless, they are expected to be approximately 1 due to the self-similarity assumption. In addition to this, closures for the SGS energies can be extracted from these terms directly by means of definition (8), i.e.,

$$
\begin{aligned}
& \hat{E}_{\mathrm{sgs}}^{\mathrm{u}, \mathrm{SS}}=\frac{1}{1} C_{21} \smile\left(\widetilde{\widetilde{\rho}} \widetilde{\widetilde{u_{k}} \widetilde{u_{k}}}-\widetilde{\widetilde{u_{k}}} \widetilde{\widetilde{u_{k}}}\right), \\
& \hat{E}_{\mathrm{sgs}}^{\mathrm{b}, \mathrm{SS}}=\frac{1}{2} C_{22}\left(\overline{\bar{B}_{k} \overline{B_{k}}}-\overline{B_{k}} \frac{\smile}{B_{k}}\right) \text {. }
\end{aligned}
$$

\section{Nonlinear closures}

Nonlinear (NL) closures are structural in nature. While they are related to other gradient (also known as tensordiffusivity) closures ${ }^{30}$ they are not based on the expansion of the primary quantities, but can be derived through gradient expansion of the filter kernel. ${ }^{31}$ In contrast to the other two families, the assumptions here are rooted in the properties of the filtering operator and not of turbulence as such. 
Truncating the expansion at first order to the following expressions: ${ }^{23}$

$$
\begin{aligned}
& \mathrm{NL}^{\mathrm{u}}: \quad \hat{\tau}_{i j}^{\mathrm{u}}=\frac{1}{12} C_{24} \Delta^{2} \bar{\rho} \widetilde{u}_{i, k} \widetilde{u}_{j, k}, \\
& \mathrm{NL}^{\mathrm{b}}: \quad \hat{\tau}_{i j}^{\mathrm{b}}=\frac{1}{12} C_{25} \Delta^{2} \bar{B}_{i, k} \bar{B}_{j, k}, \\
& \mathrm{NL}^{\mathcal{E}, \rho}: \quad \widehat{\mathcal{E}}=\frac{1}{12} C_{26} \Delta^{2} \varepsilon_{i j k}\left(\widetilde{u}_{j, l} \bar{B}_{k, l}-(\ln \bar{\rho})_{, l} \widetilde{u}_{j, l} \bar{B}_{k}\right) .
\end{aligned}
$$

The electromotive force closure is proposed in Paper I for the first time. It goes beyond the previously proposed expression $^{21,22}$

$$
\mathrm{NL}^{\mathcal{E}}: \quad \widehat{\mathcal{E}}=\frac{1}{12} C_{27} \Delta^{2} \varepsilon_{i j k} \widetilde{u}_{j, l} \bar{B}_{k, l}
$$

by explicitly capturing compressible effects in the second term. As for the scale-similarity closures, the coefficients are external to the closures and meant to capture errors not inline with the closure assumptions. Thus, values around 1 are expected. Again, closures for the SGS energies can readily be written down by definition (8) as

$$
\begin{aligned}
& \hat{E}_{\mathrm{sgs}}^{\mathrm{u}, \mathrm{NL}}=\frac{1}{12} C_{24} \Delta^{2} \bar{\rho} \widetilde{u}_{k, l} \widetilde{u}_{k, l}, \\
& \hat{E}_{\mathrm{sgs}}^{\mathrm{b}, \mathrm{NL}}=\frac{1}{12} C_{25} \Delta^{2} \bar{B}_{k, l} \bar{B}_{k, l} .
\end{aligned}
$$

A normalized version of the nonlinear SGS stress tensors has been proposed in the $\mathrm{HD}^{32,33}$ case and in our previous work $^{22}$ for MHD

$$
\begin{gathered}
\mathrm{NL}^{\mathrm{u}, \mathrm{E}}: \quad \hat{\tau}_{i j}^{\mathrm{u} *}=2 C_{28} E_{\mathrm{sgs}}^{\mathrm{u}}\left(\frac{\widetilde{u}_{i, k} \widetilde{u}_{j, k}}{\widetilde{u}_{l, s} \widetilde{u}_{l, s}}-\frac{1}{3} \delta_{i j}\right), \\
\mathrm{NL}^{\mathrm{b}, \mathrm{E}}: \quad \hat{\tau}_{i j}^{\mathrm{b} *}=2 C_{29} E_{\mathrm{sgs}}^{\mathrm{b}}\left(\frac{\bar{B}_{i, k} \bar{B}_{j, k}}{\bar{B}_{l, s} \bar{B}_{l, s}}-\frac{1}{3} \delta_{i j}\right) .
\end{gathered}
$$

Effectively, the strength is locally determined by the SGS energy, and the structural information is extracted from the unnormalized closures $\mathrm{NL}^{\mathrm{u}}$ and $\mathrm{NL}^{\mathrm{b}}$. Like the energy-scaled closures within the eddy-dissipation family, (41) and (42) are not closed. For this reason, the $E_{\mathrm{sgs}}^{\mathrm{u}}$ and $E_{\mathrm{sgs}}^{\mathrm{b}}$ can be replaced by the energy closure (13) resulting in

$$
\begin{aligned}
& \mathrm{NL}^{\mathrm{u}, \mathcal{S}^{*}}: \hat{\tau}_{i j}^{\mathrm{u} *}=2 C_{30} \hat{E}_{\mathrm{sgs}}^{\mathrm{u}, \mathcal{S}^{*}}\left(\frac{\widetilde{u}_{i, k} \widetilde{u}_{j, k}}{\widetilde{u}_{l, s} \widetilde{u}_{l, s}}-\frac{1}{3} \delta_{i j}\right), \\
& \mathrm{NL}^{\mathrm{b}, \mathcal{M}}: \quad \hat{\tau}_{i j}^{\mathrm{b} *}=2 C_{31} \hat{E}_{\mathrm{sgs}}^{\mathrm{b}, \mathcal{M}}\left(\frac{\bar{B}_{i, k} \bar{B}_{j, k}}{\bar{B}_{l, s} \bar{B}_{l, s}}-\frac{1}{3} \delta_{i j}\right) .
\end{aligned}
$$

\section{VERIFICATION METHOD}

In a first investigation, ${ }^{22}$ we analyzed the supersonic regime in simulations at a resolution of $512^{3}$ grid points. Here, we extend the parameter space to include the subsonic and hypersonic regimes, as well as two additional reference runs at a resolution of $1024^{3}$ grid points. Furthermore, the functional analysis now goes beyond the turbulent energy cascade - we also include the cross-helicity cascade and the total SGS flux of both resolved energy and cross-helicity. Finally, the structural analysis now covers alignment and magnitude of the SGS vectors, and topological properties of the SGS stresses.

\section{A. Simulations}

In total, 15 homogeneous, isotropic turbulence simulations in a periodic box with varying sonic Mach number $\mathrm{M}_{\mathrm{s}}$, Alfvénic Mach number $M_{a}$, and numerical scheme were conducted. All simulations start with uniform initial conditions, i.e., $\rho_{0}=1, \boldsymbol{u}_{0}=\mathbf{0}$ (these and all following variables are in dimensionless code units) within a box of length $L=1$ at resolution of $512^{3}$ or $1024^{3}$ grid points. The initial background magnetic field is uniform in the $\mathrm{z}$-direction and its magnitude specified by the ratio of thermal to magnetic pressure $\beta_{\mathrm{p}}=2 p / B^{2}$. The MHD equations for a compressible fluid are then evolved in time using either ENzo ${ }^{34}$ or FLASHv4. ${ }^{35}$ Statistically stationary turbulence is driven by a stochastic forcing field generated by an Ornstein-Uhlenbeck process. ${ }^{36}$ The strength is defined by a characteristic Mach number $V$. We choose a parabolic forcing profile peaking at wavenumber $k=2$ and a ratio of compressive to solenoidal components $\zeta=|\nabla \cdot \boldsymbol{u}| /\|\nabla \boldsymbol{u}\|$ for which we explore values of 0.5 and 0.9. Details on the forcing can be found in Refs. 37 and 38 , and details about individual simulation parameters are listed in Table I. In ENZO, an open-source fluid code, the ideal $(\nu=\eta=0)$ MHD equations are solved with a MUSCLHancock $^{39}$ framework, employing the second order RungeKutta integration in time, PLM reconstruction, and HLL or HLLD Riemann solvers. ${ }^{40}$ The thermal pressure $p$ is specified by an ideal equation of state with adiabatic exponent $\kappa=1.001$ to resemble an isothermal fluid. In the simulations conducted with the publicly available FLASHv4 code, the MHD equations are evolved with explicit ${ }^{41,42}$ viscosity $\nu$ and resistivity $\eta$ specified via the kinetic Reynolds number $\mathrm{Re}=\frac{L_{0} V_{0}}{\nu}=3780$ and the magnetic Reynolds number $\mathrm{Rm}=\frac{L_{0} V_{0}}{\eta}=3780$. In all simulations, the characteristic length $L_{0}^{\eta}=0.5 L$ is half the box size due to the forcing profile and the characteristic velocity $V_{0}=V c_{s, 0}$ corresponds to the forcing Mach number $V$ relative to the initial speed-ofsound $c_{s, 0}=1$. In contrast to Enzo, the gas is kept exactly isothermal by a polytropic equation of state. The chosen numerical scheme consists of second-order integration in time and space with the HLL3R Riemann solver. ${ }^{43}$ For both Enzo and FLASHv4, the divergence constraint $\nabla \cdot \boldsymbol{B}=0$ is handled by a divergence cleaning scheme. ${ }^{44}$

All simulations initially undergo a transient phase in which the uniform initial conditions evolve into stationary turbulence. This phase lasts for $t<2 T$ dynamical times with $T=0.5 \mathrm{~L} / \mathrm{V}$. Afterwards, the gas is evolved for three additional dynamical times, and ten snapshots per dynamical time are captured for the analysis. The resulting parameter space of the simulations in terms of the temporal mean $\left(\langle\square\rangle_{t}\right)$ sonic $\left\langle\left\langle\mathrm{M}_{\mathrm{s}}^{2}\right\rangle^{1 / 2}\right\rangle_{t}$ and Alfvénic $\left\langle\left\langle\mathrm{M}_{\mathrm{a}}^{2}\right\rangle^{1 / 2}\right\rangle_{t}$ spatial root mean square $(\langle\square\rangle)$ Mach numbers within $2 T<t<5 T$ is illustrated in Figure 1. Simulations 1, 2a, 4, 6, 7a, 11, 12, 13 
TABLE I. Overview of analyzed simulations. The sonic $\mathrm{M}_{\mathrm{s}}$ and Alfvénic $\mathrm{M}_{\mathrm{a}}$ Mach numbers are the temporal means of the spatial RMS numbers over the stationary phase between $2 T<t<5 T$ dynamical times. In all Enzo simulations, the ideal MHD equations were solved with an ideal equation of state. For FLASHv4 a polytropic equation of state and explicit viscosity and resistivity (so that $\mathrm{Re}=\mathrm{Rm}=3780$, see Subsection III A) was used.

\begin{tabular}{|c|c|c|c|c|c|c|c|c|}
\hline Name & Resolution & Forcing Mach $V$ & Init. $\beta_{\mathrm{p}}$ & $\left\langle\left\langle\mathrm{M}_{\mathrm{s}}^{2}\right\rangle^{1 / 2}\right\rangle$ & $\left\langle\left\langle\mathrm{M}_{\mathrm{a}}^{2}\right\rangle^{1 / 2}\right\rangle$ & Code & Riemann solver & $\zeta$ \\
\hline 1 & $512^{3}$ & 0.2 & 450 & 0.22 & 1.95 & Enzo & HLLD & 0.5 \\
\hline $2 a$ & $512^{3}$ & 0.5 & 72 & 0.56 & 1.85 & Enzo & HLLD & 0.5 \\
\hline $2 b$ & $1024^{3}$ & 0.5 & 72 & 0.57 & 1.81 & Enzo & HLLD & 0.5 \\
\hline 3 & $512^{3}$ & 0.5 & 8 & 0.61 & 1.26 & Enzo & HLLD & 0.5 \\
\hline 4 & $512^{3}$ & 1.0 & 18 & 1.17 & 1.90 & Enzo & HLLD & 0.5 \\
\hline 5 & $512^{3}$ & 1.0 & 2 & 1.25 & 1.27 & Enzo & HLLD & 0.5 \\
\hline 6 & $512^{3}$ & 2.0 & 5 & 1.97 & 2.64 & FLASHv4 & HLL3R & 0.5 \\
\hline $7 \mathrm{a}$ & $512^{3}$ & 2.0 & 5 & 2.46 & 2.14 & Enzo & HLL & 0.5 \\
\hline $7 b$ & $1024^{3}$ & 2.0 & 5 & 2.55 & 2.13 & Enzo & HLL & 0.5 \\
\hline 8 & $512^{3}$ & 2.9 & 0.25 & 2.54 & 0.78 & Enzo & HLL & 0.9 \\
\hline 9 & $512^{3}$ & 2.9 & 2.5 & 2.64 & 3.11 & ENZO & HLL & 0.9 \\
\hline 10 & $512^{3}$ & 2.9 & 25 & 2.68 & 8.24 & ENZO & HLL & 0.9 \\
\hline 11 & $512^{3}$ & 4.0 & 1 & 4.14 & 2.88 & FLASHv4 & HLL3R & 0.5 \\
\hline 12 & $512^{3}$ & 10.0 & 0.2 & 10.04 & 2.25 & FLASHv4 & HLL3R & 0.5 \\
\hline 13 & $512^{3}$ & 20.0 & 0.05 & 20.12 & 2.08 & FLASHv4 & HLL3R & 0.5 \\
\hline
\end{tabular}

within the gray area have $\left\langle\left\langle\mathbf{M}_{\mathrm{a}}^{2}\right\rangle^{1 / 2}\right\rangle_{t} \approx 3$ and are therefore used for a $\mathrm{M}_{\mathrm{s}}$-dependency analysis of the different closures.

\section{B. Reference quantities}

In order to assess the quality and performance of the different closures, we conduct functional and structural a priori tests. In a priori testing, a test filter is applied to high resolution data to mimic the effect of limited resolution. The scales below the test filter are treated as unresolved scales. Owing to the explicit filtering, we not only obtain filtered quantities intended to resemble the resolved scales but also retain the sub-filter quantities intended to resemble the unresolved scales. This allows the exact calculation of SGS quantities. In the context of LES, three different filter kernels are typically used: ${ }^{11}$ the box, the Gaussian, and the sharp spectral filter. For the majority of our analysis, we use a Gaussian filter with a characteristic filter scale at a wavenumber $k=16$ for several reasons. First, $k=16$ is within a power-law regime of the energy spectra (cf. Figure 2), which satisfies the assumption of the eddy-viscosity and scale-similarity type closures. Second, it is sufficiently far away from the forcing scale

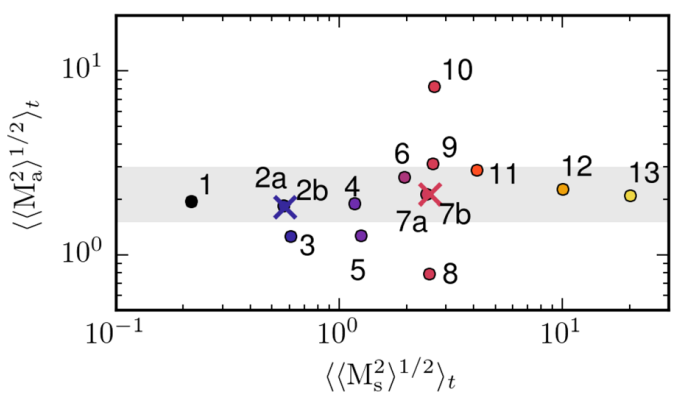

FIG. 1. Parameter space covered by the 15 simulations. Each marker (circles for a resolution of $512^{3}$ grid-points and crosses for $1024^{3}$, respectively) corresponds to the respective mean value over the stationary phase $2 T<t<$ $5 T$ of the spatial root mean square Mach numbers. Only simulations within the gray area are used in the detailed sonic Mach number dependency study. Simulation details are given in Table I.

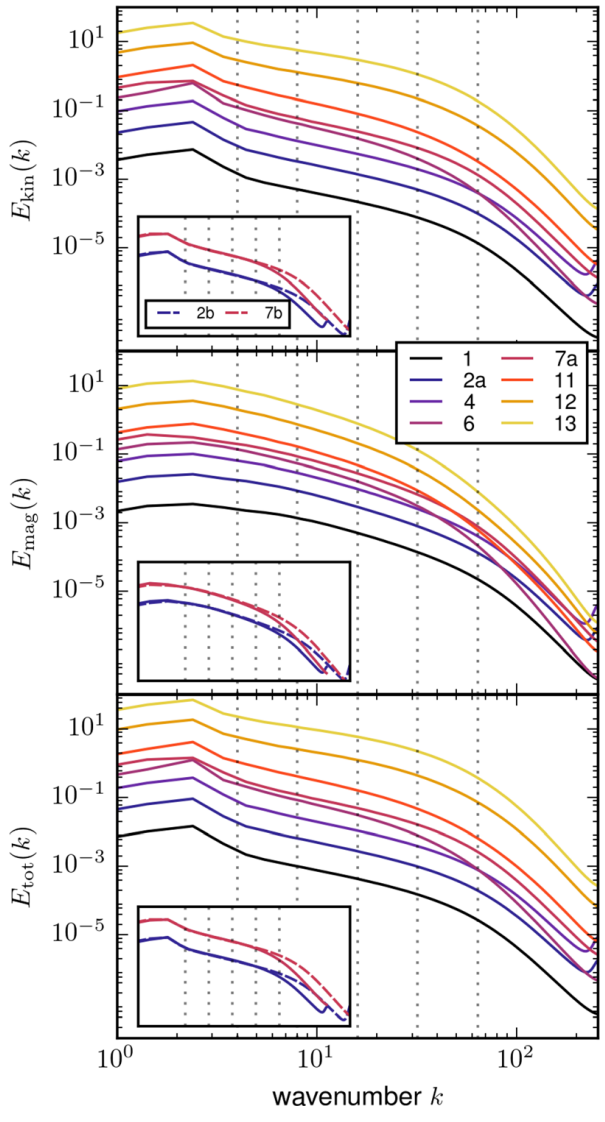

FIG. 2. Mean $(2 T<t<5 T)$ power spectra of the simulations. Kinetic energy is based on the Fourier transform of $\sqrt{\rho} \boldsymbol{u}$. The dashed vertical lines indicate the filter widths $(k=4,8,16,32$, and 64) we are using during the analysis. The insets highlight the extended power-law regime of the $1024^{3}$ runs ( $2 \mathrm{~b}$ and $7 \mathrm{~b}$, dashed lines) over the corresponding $512^{3}$ runs ( $2 \mathrm{a}$ and $7 \mathrm{a}$, solid lines). Simulation details are listed in Table I. 


\section{Paper III: A nonlinear structural subgrid-scale closure for compressible MHD. II. A priori comparison on turbulence simulation data}

$k=2$ where the dynamics of the forcing are expected to be dominant. Third, it also does not fall above the high- $k$ dropoff in the spectrum, caused by viscous and numeric dissipation, which contaminates turbulent dynamics. ${ }^{45}$ The mean spectra within the stationary regime $(2 T<t<5 T)$ of the simulations are illustrated in Figure 2, where we also highlight the filter positions. In addition to filtering at $k=16$, we also probe the closures with filter scales at $k=4,8,32,64$ to investigate the dependence of the result on the chosen scale. Moreover, we verify the results based on Gaussian filtering against a box filter. Given that we analyze compressible data, we do not employ a sharp spectral filter, which can produce negative resolved densities, and SGS stresses that violate realizability. ${ }^{25}$

The first category of tests, functional tests, probe the ability of closures to reproduce a particular (physical) property. In addition to this, they eliminate co-ordinate frame dependence by reduction to scalar diagnostics, e.g., of six SGS stress tensor or three EMF vector components. Historically, the most frequently used reference quantity is the turbulent energy flux, i.e., the cascade term

$$
\Sigma^{E}=\tau_{i j} \widetilde{\mathcal{S}}_{i j}+\mathcal{E} \cdot \bar{J}
$$

It encodes the local exchange between resolved and unresolved energy and is connected to the turbulent energy cascade. However, as it was recently shown, ${ }^{9}$ the total energy flux term

$$
\mathcal{F}^{E}=-\widetilde{\boldsymbol{u}} \cdot(\nabla \cdot \tau)+\overline{\boldsymbol{B}} \cdot \nabla \times \mathcal{E}
$$

is more strongly influenced by the transport terms $\nabla \cdot(\widetilde{\boldsymbol{u}} \cdot \tau$ $+\overline{\boldsymbol{B}} \times \mathcal{E})$ rather than the cascade term $\Sigma^{E}$ in our simulations. Furthermore, in MHD there are additional conserved quantities such as cross-helicity, $W=\boldsymbol{u} \cdot \boldsymbol{B}$, which are, in the context of LES, also governed by resolved and subgrid-scale evolution equations. ${ }^{9}$ The exchange of cross-helicity across the filter scale is analogous to the energy one, with crosshelicity flux

$$
\Sigma^{W}=\tau_{i j}\left(\overline{B_{i}} / \bar{\rho}\right)_{, j}+\mathcal{E} \cdot \widetilde{\boldsymbol{\Omega}} .
$$

Again, the total cross-helicity term

$$
\mathcal{F}^{W}=-\overline{\boldsymbol{B}} / \bar{\rho} \cdot(\nabla \cdot \tau)+\widetilde{\boldsymbol{u}} \cdot \nabla \times \mathcal{E}
$$

is dominated by the transport and not the cascade contribution. ${ }^{9}$ In the following, we are going to analyze all four (pseudo-)scalars as each of them may play a crucial role in different dynamical regimes, and systematic differences between results from total and cascade fluxes may indicate the importance of the differentiation commutator. ${ }^{23}$ Specifically, we conduct nonlinear least-square minimization $^{46}$ between data and closure. This automatically produces the best coefficient $C_{\square}$ for each snapshot and closure individually. Eventually, we calculate the Pearson correlation coefficient as an overall measure of accuracy. While these correlations probe the spatially local performance of the closures, we also analyze a global indicator. In particular, we look at the average SGS dissipation, i.e., the total $\Sigma^{E}$ for each snapshot, and examine the contributions of the individual components.

The performed structural tests start with a topological analysis. We use the geometric invariants of second-rank tensors to compare the topology of the deviatoric SGS Reynolds $\tau^{u *}$ and Maxwell $\tau^{\mathrm{b} *}$ stress tensors for data and closure. The characteristic polynomial of a second-rank tensor $\mathcal{T}$ is ${ }^{47} \lambda_{i}^{3}$ $+P \lambda_{i}^{2}+Q \lambda_{i}+R=0$ with eigenvalues $\lambda_{i}$ and invariants

$$
\begin{gathered}
P=-\operatorname{tr}(\mathcal{T})=-\left(\lambda_{1}+\lambda_{2}+\lambda_{3}\right), \\
Q=\frac{1}{2}\left(P^{2}-\operatorname{tr}\left(\mathcal{T}^{2}\right)\right)=\lambda_{1} \lambda_{2}+\lambda_{2} \lambda_{3}+\lambda_{3} \lambda_{1}, \\
R=-\operatorname{det}(\mathcal{T})=-\lambda_{1} \lambda_{2} \lambda_{3} .
\end{gathered}
$$

Both tensors, $\tau^{\mathrm{u} *}$ and $\tau^{\mathrm{b} *}$, are traceless, so $P=0$. Furthermore, they are symmetric. Thus, $Q$ is negative definite and the three eigenvalues $\lambda_{1} \geq \lambda_{2} \geq \lambda_{3}$ are real. Therefore, only two eigenvalue combinations are possible. On the one hand, sheet-like structures with $R>0$ are produced by expansion in two dimensions $\left(\lambda_{1}, \lambda_{2}>0\right)$, and contraction in the third dimension $\left(\lambda_{3}<0\right)$. On the other hand, tube-like structures with $R<0$ are produced by expansion in one dimension $\left(\lambda_{1}>0\right)$ and contraction in two dimensions $\left(\lambda_{2}, \lambda_{3}<0\right)$.

Given that all closures enter the primary equations ultimately in vectorial form, we also asses their geometrical performance. For this reason, we compare the alignment of the data vector, e.g., $\nabla \cdot \tau^{\mathrm{u} *}$, with the corresponding closure vector, i.e., $\nabla \cdot \hat{\tau}_{i j}^{\mathrm{u*}}$. Moreover, we compare their respective magnitudes. Ideally, the modeled SGS vector will point in the identical direction as the data vector $\left(\cos \left(\nabla \cdot \hat{\tau}_{i j}^{\mathrm{u} *}\right.\right.$, $\left.\nabla \cdot \tau^{\mathrm{u} *}\right)=1$ ) and will be with identical magnitude $\left(\left|\nabla \cdot \hat{\tau}_{i j}^{\mathrm{u} *}\right| /\left|\nabla \cdot \tau^{\mathrm{u} *}\right|=1\right)$.

\section{RESULTS}

\section{A. Functional analysis: Overview and $M_{s}$ dependency}

We start our functional analysis by evaluating the performance of the different closures for the isotropic parts of the SGS stresses, i.e. by definition (8), the SGS energies $\tau_{i j}^{\square}=2 / 3 \delta_{i j} E_{\mathrm{sgs}}^{\square}$. Figure 3 illustrates the creation of the Mach

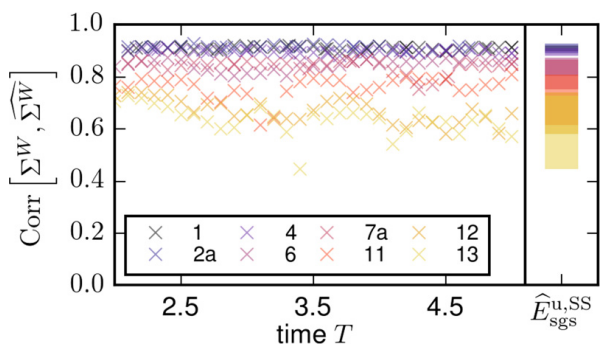

FIG. 3. Illustration of using correlations from individual simulation snapshots (left) to create a bar plot (right). Each marker on the left side corresponds to the correlation coefficient from one snapshot of the color-coded simulations from the subsonic (dark) to the hypersonic (bright) regime. The correlation coefficient is always calculated for only one reference quantity (here, the cross-helicity cascade flux $\Sigma^{W}$ ) with one closure (here, the kinetic SGS energy of the scale-similarity family $\hat{E}_{\mathrm{sgs}}^{\mathrm{u}, \mathrm{SS}}$ ). Each colored bar on the right side spans the range of variation from the minimum to the maximum correlation value over all snapshots of one simulation. 
number dependent bar plots we use in this section for one sample quantity. We use the kinetic SGS energy closure of the scale-similarity family $\hat{E}_{\mathrm{sgs}}^{\mathrm{u}, \mathrm{SS}}(33)$ and compute the correlation $\operatorname{Corr}\left[\Sigma^{W}, \widehat{\Sigma^{W}}\right]$ of the contribution to cross-helicity cascade term $\Sigma^{W}$ based on closure and exact SGS energy expression $E_{\mathrm{sgs}}^{\mathrm{u}}(7)$, i.e.,

$$
\operatorname{Corr}\left[\frac{2}{3} \delta_{i j} E_{\mathrm{sgs}}^{\mathrm{u}}\left(\overline{B_{i}} \bar{\rho}\right)_{, j}, \frac{2}{3} \delta_{i j} \hat{E}_{\mathrm{sgs}}^{\mathrm{u}, \mathrm{SS}}\left(\overline{B_{i}} \bar{\rho}\right)_{, j}\right] .
$$

This is done for each snapshot of all simulations. Then, we take the minimum and maximum value of each simulation separately to determine the vertical extent of the color-coded bars in the right panel of the figure. In this example, it is clear that the cross-helicity cascade is well modeled in the subsonic regime (with correlations above 0.8 ) and tends to perform worse in the hypersonic regime (going down to almost 0.4 ).

The results for all energy closures and all reference quantities are shown in Figure 4(a). In general, all closures perform very well with respect to the cascade fluxes. A notable exception is the already mentioned cross-helicity cascade correlation of the kinetic scale-similarity model $\hat{E}_{\mathrm{sgs}}^{\mathrm{u}, \mathrm{SS}}$, which has a strong $\mathrm{M}_{\mathrm{s}}$ dependency. In addition to this, it can be seen that the total flux terms are generally less well represented than the cascade terms. Furthermore, there is practically no difference between modeling the eddy-viscosity/ diffusivity energies based on realizability conditions $\left(\hat{E}_{\mathrm{sgs}}^{\mathrm{u}, \mathcal{S}^{*}}\right.$ and $\left.\hat{E}_{\text {sgs }}^{\mathrm{b}, \mathcal{M}}\right)$ and the equilibrium approach $\left(\hat{E}_{\mathrm{sgs}}^{\mathrm{u}, \mathcal{S}}\right.$ and $\left.\hat{E}_{\mathrm{sgs}}^{\mathrm{b}, J}\right)$. Overall, with a slight advantage over the eddy-viscosity closures, the nonlinear closures perform best with generally high correlations ( $>0.7$ across the entire parameter space) and very limited $\mathrm{M}_{\mathrm{s}}$ dependency. The median across all simulations of the free coefficient value of each closure is listed in Table II including bounds given by the interquartile range (IQR). For reference, we also provide more detailed data tables as the supplementary material. ${ }^{48}$ All SGS energy closures exhibit only a very limited spread over the tested parameter space with IQRs within a factor of 2 around the median. These results also hold (not shown) for direct fits, i.e., $\operatorname{Corr}\left[E_{\mathrm{sgs}}^{\square}, \hat{E}_{\mathrm{sgs}}^{\square}\right]$, of the kinetic $E_{\mathrm{sgs}}^{\mathrm{u}}$, magnetic $E_{\mathrm{sgs}}^{\mathrm{b}}$, and total $E_{\mathrm{sgs}}$ energies.

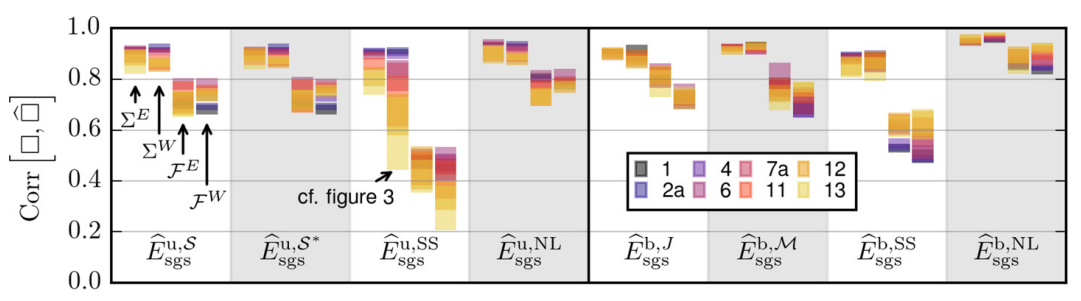

(a)Correlations of different SGS energy closures, i.e. the isotropic component of the SGS stress tensors.

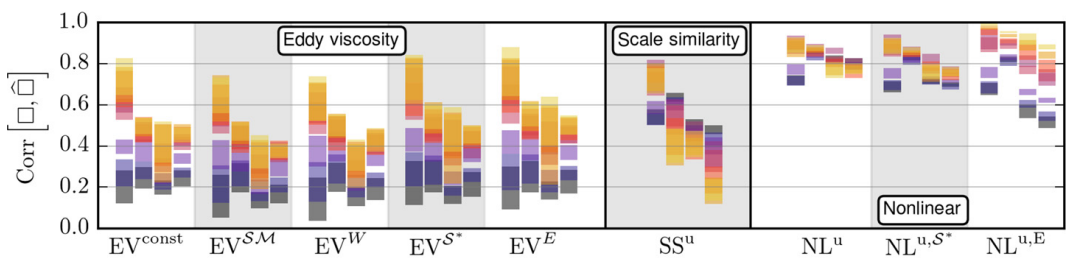

(b)Correlations of different traceless SGS Reynolds stress $\tau^{\mathrm{u} *}$ closures.

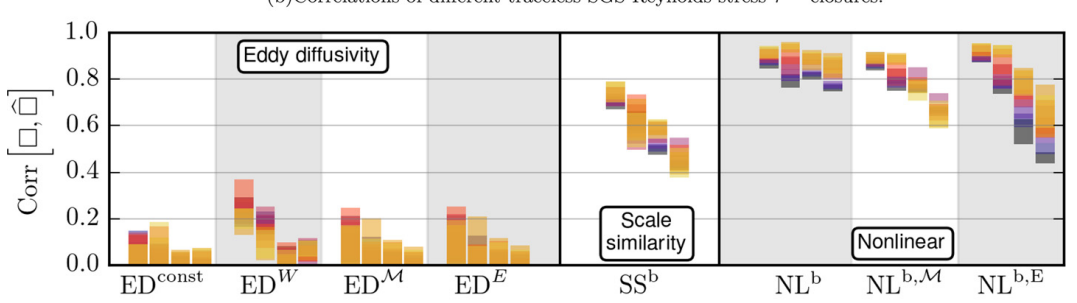

Correlations between closure and data for all reference fluxes. For each closure, the four colored bars (from left to right: energy $\Sigma^{E}$ and cross-helicity $\Sigma^{W}$ cascade, and total energy $\mathcal{F}^{E}$ and cross-helicity $\mathcal{F}^{W}$ flux) illustrate the maximum range of correlation split by simulation. A detailed explanation of the colored bars is given in Figure 3. Subsonic runs are towards the dark end and supersonic at the bright end of the palette (cf. Figure 2). All simulations have been filtered at $k=16$. The $\mathrm{x}$-axis labels denote the different closure identifiers as introduced in Section II.

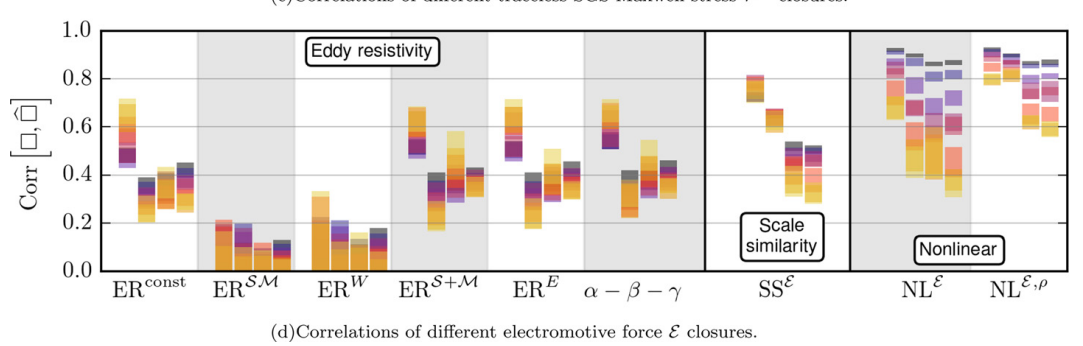




\section{Paper III: A nonlinear structural subgrid-scale closure for compressible MHD. II. A priori comparison on turbulence simulation data}

TABLE II. Median correlation and coefficient values over all $512^{3}$ simulations filtered at $k=16$ with lower and upper bound given by interquartile range of all data. Please note that for the nested closures, e.g., $\mathrm{EV}^{\mathcal{S}^{*}}$, the listed coefficient contains both coefficients. Detailed data tables including results split by reference quantity and min-/maximum values can be found in the supplementary material. ${ }^{48}$

\begin{tabular}{|c|c|c|}
\hline ID & $\operatorname{Corr}[\square, \hat{\square}]$ & Coefficient \\
\hline$\hat{E}_{\mathrm{sgs}}^{\mathrm{u}, \mathcal{S}}$ & $0.83_{-0.094}^{+0.082}$ & $C_{4}=0.036_{-0.0074}^{+0.014}$ \\
\hline$\hat{E}_{\mathrm{sgs}}^{\mathrm{u}, \mathcal{S}^{*}}$ & $0.84_{-0.098}^{+0.068}$ & $C_{2}=0.038_{-0.005}^{+0.022}$ \\
\hline$\hat{E}_{\mathrm{sgs}}^{\mathrm{u}, \mathrm{SS}}$ & $0.59_{-0.11}^{+0.3}$ & $C_{21}=1_{-0.35}^{+0.19}$ \\
\hline$\hat{E}_{\mathrm{sgs}}^{\mathrm{u}, \mathrm{NL}}$ & $0.85_{-0.058}^{+0.077}$ & $C_{24}=1.2_{-0.19}^{+0.53}$ \\
\hline$\hat{E}_{\mathrm{sgs}}^{\mathrm{b}, J}$ & $0.83_{-0.084}^{+0.057}$ & $C_{3}=0.043_{-0.0065}^{+0.021}$ \\
\hline$\hat{E}_{\mathrm{sgs}}^{\mathrm{b}, \mathcal{M}}$ & $0.87_{-0.13}^{+0.054}$ & $C_{1}=0.045_{-0.0058}^{+0.028}$ \\
\hline$\hat{E}_{\mathrm{sgs}}^{\mathrm{b}, \mathrm{SS}}$ & $0.79_{-0.23}^{+0.093}$ & $C_{22}=1.1_{-0.36}^{+0.26}$ \\
\hline$\hat{E}_{\mathrm{sgs}}^{\mathrm{b}, \mathrm{NL}}$ & $0.93_{-0.073}^{+0.018}$ & $C_{25}=1.3_{-0.1}^{+0.43}$ \\
\hline $\mathrm{EV}^{\text {const }}$ & $0.4_{-0.12}^{+0.079}$ & $C_{5}=0.096_{-0.074}^{+0.13}$ \\
\hline $\mathrm{EV}^{\mathcal{S M}}$ & $0.35_{-0.1}^{+0.081}$ & $C_{8}=0.011_{-0.0057}^{+0.0061}$ \\
\hline $\mathrm{EV}^{W}$ & $0.39_{-0.12}^{+0.092}$ & $C_{10}=0.024_{-0.008}^{+0.008}$ \\
\hline $\mathrm{EV}^{\mathcal{S}^{*}}$ & $0.43_{-0.13}^{+0.091}$ & $C_{16}=0.0085_{-0.0031}^{+0.0031}$ \\
\hline $\mathrm{EV}^{E}$ & $0.44_{-0.15}^{+0.089}$ & $C_{13}=0.041_{-0.021}^{+0.017}$ \\
\hline $\mathrm{ED}^{\text {const }}$ & $0.02_{-0.012}^{+0.016}$ & $C_{6}=0.00071_{-0.006}^{+-0.006}$ \\
\hline $\mathrm{ED}^{W}$ & $0.089_{-0.042}^{+0.1}$ & $C_{11}=-0.0066_{-0.0067}^{+0.0025}$ \\
\hline $\mathrm{ED}^{\mathcal{M}}$ & $0.026_{-0.012}^{+0.021}$ & $C_{17}=0.00014_{-0.00038}^{+0.00014}$ \\
\hline $\mathrm{ED}^{E}$ & $0.027_{-0.014}^{+0.02}$ & $C_{14}=0.00055_{-0.002}^{+0.00093}$ \\
\hline $\mathrm{ER}^{\text {const }}$ & $0.35_{-0.053}^{+0.092}$ & $C_{7}=0.14_{-0.11}^{+0.054}$ \\
\hline $\mathrm{ER}^{\mathcal{S M}}$ & $0.032_{-0.017}^{+0.024}$ & $C_{9}=-0.00055_{-0.0015}^{+0.0011}$ \\
\hline $\mathrm{ER}^{W}$ & $0.042_{-0.024}^{+0.035}$ & $C_{12}=-0.0014_{-0.0039}^{+0.0021}$ \\
\hline $\mathrm{ER}^{\mathcal{S}+\mathcal{M}}$ & $0.36_{-0.057}^{+0.11}$ & $C_{18}=0.0096_{-0.0035}^{+0.0068}$ \\
\hline $\mathrm{ER}^{E}$ & $0.36_{-0.056}^{+0.1}$ & $C_{15}=0.035_{-0.013}^{+0.025}$ \\
\hline$\alpha-\beta-\gamma$ & $0.37_{-0.049}^{+0.11}$ & $\left\{\begin{array}{l}C_{20}=-0.0026_{-0.0043}^{+0.0018} \\
C_{15}=0.033_{-0.0087}^{+0.028} \\
C_{19}=-0.00017_{-0.0079}^{+0.0058}\end{array}\right.$ \\
\hline $\mathrm{SS}^{\mathrm{u}}$ & $0.49_{-0.072}^{+0.11}$ & $C_{21}=0.67_{-0.23}^{+0.16}$ \\
\hline $\mathrm{SS}^{\mathrm{b}}$ & $0.58_{-0.084}^{+0.081}$ & $C_{22}=0.9_{-0.43}^{+0.25}$ \\
\hline $\mathrm{SS}^{\mathcal{E}}$ & $0.55_{-0.084}^{+0.13}$ & $C_{23}=0.89_{-0.18}^{+0.098}$ \\
\hline $\mathrm{NL}^{\mathrm{u}}$ & $0.82_{-0.029}^{+0.038}$ & $C_{24}=0.98_{-0.19}^{+0.081}$ \\
\hline $\mathrm{NL}^{\mathrm{u}, \mathcal{S}^{*}}$ & $0.77_{-0.038}^{+0.069}$ & $C_{30}=0.032_{-0.0052}^{+0.0026}$ \\
\hline $\mathrm{NL}^{\mathrm{u}, \mathrm{E}}$ & $0.81_{-0.13}^{+0.078}$ & $C_{28}=0.52_{-0.12}^{+0.09}$ \\
\hline $\mathrm{NL}^{\mathrm{b}}$ & $0.85_{-0.038}^{+0.029}$ & $C_{25}=1.1_{-0.063}^{+0.19}$ \\
\hline $\mathrm{NL}^{\mathrm{b}, \mathcal{M}}$ & $0.77_{-0.074}^{+0.065}$ & $C_{31}=0.039_{-0.0052}^{+0.0093}$ \\
\hline $\mathrm{NL}^{\mathrm{b}, \mathrm{E}}$ & $0.76_{-0.14}^{+0.11}$ & $C_{29}=0.52_{-0.21}^{+0.21}$ \\
\hline $\mathrm{NL}^{\mathcal{E}}$ & $0.7_{-0.13}^{+0.13}$ & $C_{27}=1.2_{-0.11}^{+0.14}$ \\
\hline $\mathrm{NL}^{\mathcal{E}, \rho}$ & $0.84_{-0.072}^{+0.04}$ & $C_{26}=1_{-0.3}^{+0.11}$ \\
\hline
\end{tabular}

The correlations of all four functional reference quantities for the traceless SGS Reynolds stress $\hat{\tau}_{i j}^{\mathrm{u} *}$ are depicted in Figure 4(b). All eddy-viscosity type closures EV ${ }^{\square}$ are very similar and insensitive to the scaling chosen. Even though the correlations for all snapshots within a single simulation do not vary much, there is a substantial difference between the simulations. Correlations are typically below 0.2 in the subsonic regime whereas they can reach $>0.8$ in the highly supersonic regime. This ordering is present in all reference quantities for the EV closures. The scale-similarity SS ${ }^{\mathrm{u}}$ closure also exhibits this behavior for the turbulent energy cascade $\Sigma^{E}$ even though the lower bound in the subsonic regime is much better, $\approx 0.5$. However, the correlations of the other fluxes, $\Sigma^{W}, \mathcal{F}^{E}$, and $\mathcal{F}^{W}$, have the opposite ordering. The most extreme case of $\mathcal{F}^{W}$ spreads from $\approx 0.5$ in the subsonic regime to correlations as low as 0.1 for the $\mathrm{M}_{\mathrm{s}} \approx 20$ simulation. The nonlinear family NL is closest to the data in general. Again, we observe an ordering with $\mathrm{M}_{\mathrm{s}}$ but the spread is much more constrained and for $\mathrm{NL}^{\mathrm{u}}$ the correlations are consistently above 0.7 . Here, the scaling only further separates individual simulations with supersonic simulations slightly improving and subsonic simulations becoming worse on average.

Generally, the results for the traceless SGS Maxwell stress $\hat{\tau}_{i j}^{\mathrm{b} *}$, as shown in Figure 4(c), are very similar to those for $\hat{\tau}_{i j}^{\mathrm{u} *}$. Again, the nonlinear family has the best performance and different normalizations for NL cause a wider spread. The scale-similarity closure $\mathrm{SS}^{\mathrm{b}}$ is slightly worse with best correlations up to 0.8 for $\Sigma^{E}$ and worst $(0.4)$ for $\mathcal{F}^{W}$. Most striking is the poor performance of all eddy-diffusivity (ED) closures. Independent of normalization and simulation, the correlations barely reach 0.4 with the majority of snapshots (93\%) being below 0.2 for all reference quantities.

Finally, the findings for the electromotive force $\mathcal{E}$ are much more diverse. First, within the eddy-resistivity (ER) family, scaling by cross-helicity leads to poor correlations (99\% snapshots below 0.2). However, ER ${ }^{\text {const }}$ and energy scalings $\left(\mathrm{ER}^{\mathcal{S M}}\right.$ and $\mathrm{ER}^{E}$ ) provide reasonable correlations (from 0.5 for low $\mathrm{M}_{\mathrm{s}}$ to 0.7 for high $\mathrm{M}_{\mathrm{s}}$ ) for the turbulent energy cascade $\Sigma^{E}$ but are less effective $(<0.5)$ for $\Sigma^{W}, \mathcal{F}^{E}$, and $\mathcal{F}^{W}$. In addition, there is practically no difference between these scalings and the addition of the two extra terms in the $\alpha-\beta-\gamma$ closure. Second, the scale-similarity closure $\mathrm{SS}^{\mathcal{E}}$ performs similar to the reasonable ER closures with respect to the total terms $\mathcal{F}^{E}$ and $\mathcal{F}^{W}$. However, it performs much better for the cascade terms with correlations for $\Sigma^{W}$ of $\approx 0.65$ and for $\Sigma^{E}$ of $\approx 0.75$ without significant $\mathrm{M}_{\mathrm{s}}$ dependence. Third, the effect of the compressible extension of the nonlinear closure $\mathrm{NL}^{\mathcal{E}, \rho}$ becomes apparent when comparing the results for different simulations. While there is practically no difference between $\mathrm{NL}^{\mathcal{E}}$ and $\mathrm{NL}^{\mathcal{E}, \rho}$ in the subsonic regime (correlations $>0.9$ for all quantities), the shortcomings of $\mathrm{NL}^{\mathcal{E}}$ in the highly supersonic regime are apparent. Correlations of $\approx 0.4$ for $\Sigma^{W}, \mathcal{F}^{E}$, and $\mathcal{F}^{W}$ in the $\mathrm{M}_{\mathrm{s}} \approx 20$ simulation can be improved by the additional term in $\mathrm{NL}^{\mathcal{E}, \rho}$ to $\approx 0.6$ for $\mathcal{F}^{E}$ and $\mathcal{F}^{W}$, and even up to $\approx 0.8$ for $\Sigma^{W}$. The improvements for $\mathrm{NL}^{\mathcal{E}, \rho}$ are more pronounced in the cascade terms (with a spread of $0.8-0.9$ ) than in the total flux terms (with a spread of 0.6-0.9). Here, the additional differentiation commutator ${ }^{23}$ might further increase the correlations in the high- $\mathrm{M}_{\mathrm{s}}$ regime. The overall trend that the nonlinear closures are better correlated with the data than the scalesimilarity or eddy-resistivity closures continues for the electromotive force as well.

Furthermore, as listed in Table II, closures that exhibit a generally high correlation show the least spread in their free coefficient values $C_{\square}$ and vice versa. For example, $\mathrm{NL}^{\mathrm{u}}$, 
with a median correlation of 0.82 , has a spread in the coefficient value of $<20 \%$. In contrast to this, $\mathrm{ED}^{E}$, with a median correlation of 0.027 , has median coefficient of effectively 0 because it takes both negative and positive values. It should be noted that all scale-similarity closures and the unnormalized nonlinear closures have coefficients of $C_{\square} \approx 1$, as expected analytically. Finally, the common coefficient $C_{15}$, which the $\alpha-\beta-\gamma$ and $\mathrm{ER}^{E}$ closures share, is essentially identical, while the two additional terms in the $\alpha-\beta-\gamma$ closure are effectively canceled by their free coefficients $C_{19}, C_{20} \approx 0$. This also explains their identical behavior in correlations.

\section{B. Functional analysis: Filter widths and kernel shapes}

In subsection IV A, we saw that the differences in correlations for functional tests are most pronounced between closure families and that normalization within a family itself is subdominant. For this reason, we continue our analysis with the best performing closure of each family. In this section, we verify that the results shown in subsection IV A from simulations at a resolution of $512^{3}$ filtered at $k=16$ do not substantially change with resolution and we investigate how the different closures react to the chosen filter scale.

Figure 5 illustrates the comparison of correlation and coefficient values among four simulations (2a,b and $7 \mathrm{a}, \mathrm{b}$ ) that differ in driving (subsonic and supersonic) and resolution $\left(512^{3}\right.$ and $\left.1024^{3}\right)$. Furthermore, we apply the filter at different scales $k=4,8,16,32$, and 64 . The extreme cases, $k=4$ and $k=64$, are very close to the forcing regime or already in the dissipation regime, ${ }^{45}$ respectively. Generally, we confirm the observed ordering in correlations among closure families described in subsection IV A. Independent of resolution and filter width, the nonlinear closures outperform the scale-similarity and eddy-viscosity type closures. On average the difference in both correlations and coefficient values between the $512^{3}$ and $1024^{3}$ simulations are below $7 \%$ at $k=16$. Furthermore, all closures typically achieve higher

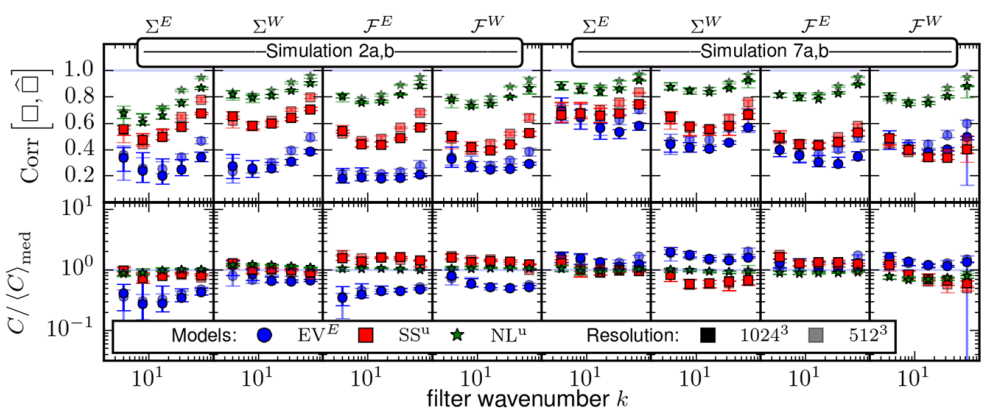

(a) SGS Reynolds stress closures $\widehat{\tau}_{i j}^{\mathrm{u} *}$

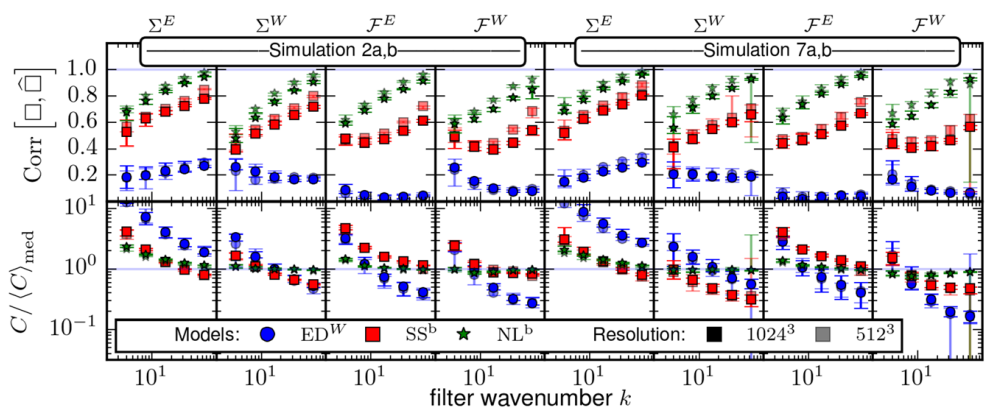

(b) SGS Maxwell stress closures $\widehat{\tau}_{i j}^{b *}$

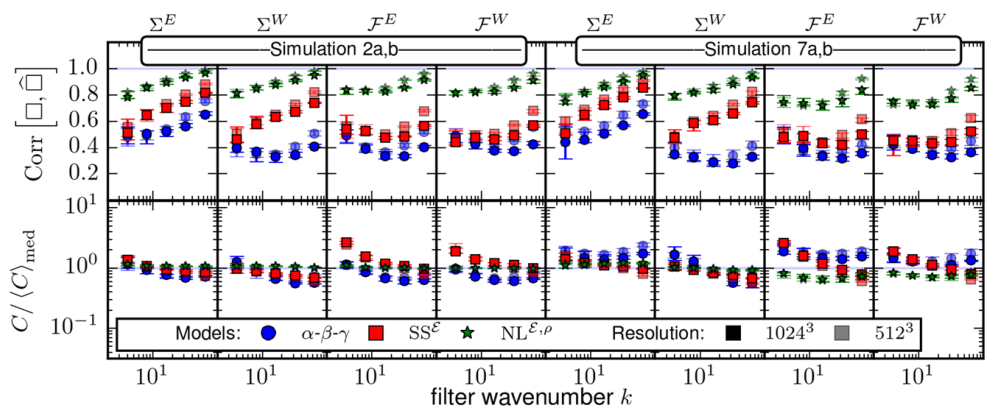

(c) Electromotive force closures $\widehat{\mathcal{E}}$
FIG. 5. Comparison of the median correlation (top row in each plot) and coefficient (bottom row) value at different filter wavenumbers $k=$ $4,8,16,32$, and 64 and simulation resolutions $512^{3}$ (transparent) and $1024^{3}$ (opaque) for subsonic simulation $2 \mathrm{a}, \mathrm{b}$ and supersonic simulation 7a,b. The error bars illustrate the respective minimum and maximum values. Each column corresponds to results of fitting one reference quantity $\Sigma^{E}, \Sigma^{W}, \mathcal{F}^{E}$, or $\mathcal{F}^{W}$, and each marker represents the median value over snapshots at $t=$ $\{2,2.5,3,3.5,4,4.5,5\} T$ of the particular simulation. The coefficient values are normalized to the respective median value over the snapshots of both simulations and at all filter widths at a given resolution. 
correlations $(\approx 25 \%$ while filtering at $k=64$ compared to $k=16)$ towards the high $k$ end and the correlations from $512^{3}$ simulations at $k>16$ tend to be higher than from simulations at $1024^{3}$. This is not surprising. On the one hand, the amount of subgrid-scale dynamics that needs to be modeled is reduced with increasing filter wavenumber. One the other hand, there is less physical information at high $k$ for lower resolutions. Nevertheless, for some cases, there are more subtle differences with respect to filter scale, which we describe in the following.

In Figure 5(a), the best closures within each family for the SGS Reynolds stress are shown, i.e., $\mathrm{EV}^{E}, \mathrm{SS}^{\mathrm{u}}$, and $\mathrm{NL}^{\mathrm{u}}$. The overall correlation, depending on filter scale $k$, for each model and reference quantity has a very shallow U-shape. Compared to $k=16$, the correlations are $\approx 6 \%$ higher at $k=4$ and $\approx 30 \%$ higher at $k=64$, respectively. The slight increase at $k=4$ might be attributed to the proximity to the forcing scale $k \approx 2$, which is completely resolved. Thus, the largest unresolved scales of $\tau^{\mathrm{u}}$ might see an imprint of the (resolved) forcing and lack SGS turbulent dynamics, which, in turn, renders specific SGS modeling unnecessary and increases the correlation. The observed systematic differences in correlations with varying $k$ are generally not present in the coefficient values. However, the values vary to different extents within each family and reference quantity. While the mean deviation from the median coefficient over all reference quantities, filter widths, and snapshots is only $10 \%$ for the nonlinear closure $\mathrm{NL}^{\mathrm{u}}$, it varies by $47 \%$ for the eddy-viscosity reference closure $\mathrm{EV}^{E}$. Compared to the results of $\tau^{\mathrm{b} *}$ in the next paragraph, this is still acceptable, even though we find systematically lower coefficient at $\mathrm{M}_{\mathrm{s}} \approx 0.6$ compared to $\mathrm{M}_{\mathrm{s}} \approx 2.5$.

The SGS Maxwell stress results depicted in Figure 5(b) show a strong filter scale dependency of the closure coefficient for the scale-similarity $\mathrm{SS}^{\mathrm{b}}$ and eddy-diffusivity $\mathrm{ED}^{W}$ closure. The coefficients are larger for small $k$ and decrease with increasing $k$ spanning almost two orders-of-magnitude. Only the nonlinear closure $\mathrm{NL}^{\mathrm{b}}$ keeps a rather constant value with deviations of $17 \%$ on average. The correlations, on the other hand, show a systematic increase with $k$ for $\mathrm{NL}^{\mathrm{b}}$ in all reference quantities. This might be ascribed to the absence of a direct forcing term acting on the magnetic field. Similar

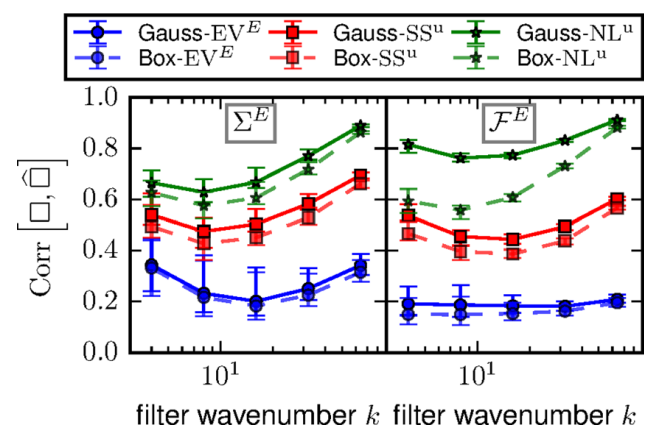

FIG. 6. Correlations of the energy cascade, $\Sigma^{E}$, and total energy, $\mathcal{F}^{E}$, flux of different deviatoric kinetic SGS stress closures for different filter widths and kernels (box - - and Gaussian -) in subsonic simulation 2b. Markers indicate the median, and the error bars show the minimum and maximum value over time. behavior is also present in $\mathrm{SS}^{\mathrm{b}}$ with the slight difference of a plateau for $k \leq 16$ in the total flux quantities $\mathcal{F}^{E}$ and $\mathcal{F}^{W}$. Finally, the eddy-diffusivity closure never reaches a correlation higher than 0.36 over the entire parameter space.

The different closures for the electromotive force $\mathcal{E}$ are closer to each other as illustrated in Figure 5(c). Here, both $\mathrm{NL}^{\mathcal{E}, \rho}$ and $\mathrm{SS}^{\mathcal{E}}$ exhibit strictly increasing correlation values with $k$ for the cascade terms $\Sigma^{E}$ and $\Sigma^{W}$ and a plateau for $k \leq 16$ for the total flux terms $\mathcal{F}^{E}$ and $\mathcal{F}^{W}$. The coefficient values for all $\mathcal{E}$ closure are less widely spread. The $\alpha-\beta-\gamma$ closure has a variation of $37 \%$ around the median over all data whereby we only take the dominant $\beta$ term into account. The $\mathrm{SS}^{\mathcal{E}}$ closure has a variation of $47 \%$, and the nonlinear closure is effectively constant with a spread of only $16 \%$.

Finally, the differences between using a Gaussian and a box kernel for the analysis are illustrated in Figure 6. Two trends can be observed for the kinetic energy cascade and total flux. The correlations of $\Sigma^{E}$ for the box filter are (within the error bars) slightly lower $(\$ 10 \%)$ for all models and filter widths. In addition, the correlations exhibit a more pronounced deviation for the total energy flux $\mathcal{F}^{E}$ especially at smaller filter wavenumbers $k$ and thus larger filter widths. We attribute this to the non-smooth nature of the box kernel versus the Gaussian kernel resulting in numerical biases in the computation of gradient-based quantities. This could explain why the deviations are more pronounced in the total flux which has an additional divergence operator acting on the SGS terms in comparison to the cascade flux. Likewise, the effect would be more pronounced in the nonlinear closures as they are built from nonlinear combinations of gradients. The observed convergence between box and Gaussian filtering with increasing $k$ is also expected, because the differences between the kernels become less distinct for small widths. Overall, the observed behavior based on Gaussian filtering, i.e., better performance of the nonlinear closures over the scale-similarity and the eddy-dissipation family ones, also holds for filtering with a box kernel. These trends similarly apply to the cross-helicity fluxes and other SGS terms, too.

\section{Functional analysis: Average SGS dissipation}

We close the functional analysis with a comparison of the contributions by individual components to the average SGS dissipation $\Sigma^{E}$. Figure 7 illustrates the share of deviatoric kinetic SGS stress, $\tau_{i j}^{\mathrm{u} *} \widetilde{\mathcal{S}}_{i j}$, and deviatoric magnetic SGS stress, $\tau_{i j}^{\mathrm{b} *} \widetilde{\mathcal{S}}_{i j}$, kinetic SGS pressure, $1 / 3 \tau_{k k}^{\mathrm{u}} \widetilde{\mathcal{S}}_{k k}$, and magnetic SGS pressure, $1 / 6 \tau_{k k}^{\mathrm{b}} \widetilde{\mathcal{S}}_{k k}$, and EMF, $\mathcal{E} \cdot \overline{\mathbf{J}}$ to $\Sigma^{E}$ for different filter widths. In general, both SGS pressures (and thus energies) are almost negligible $(<10 \%)$ in the reference data even though the data cover the slightly supersonic regime (simulation 7b). Similarly, the deviatoric kinetic SGS stress is subdominant $(10 \%-20 \%)$ while the deviatoric magnetic SGS stress and the EMF jointly contribute $\approx 80 \%$ to the total SGS dissipation independent of the chosen filter scale. While the magnetic stress dominates at the largest scales (up to $50 \%$ at $k=4$ ), its contribution constantly decreases, and at the smallest scale the EMF is strongest reaching a contribution of $\approx 50 \%$. This can be understood by analyzing the ratio 


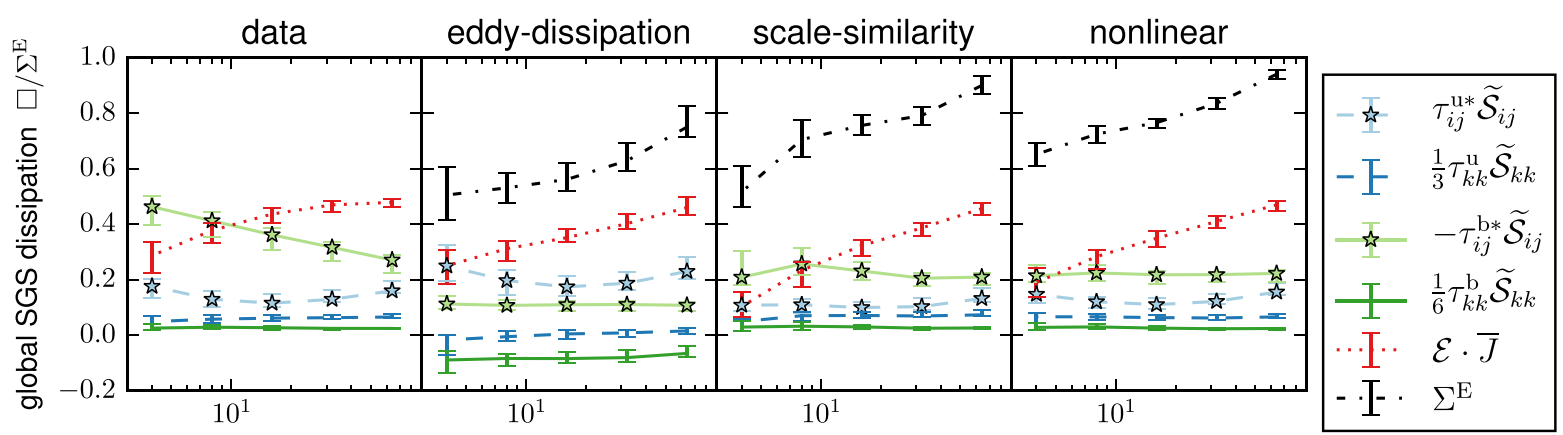

filter wavenumber $k \quad$ filter wavenumber $k \quad$ filter wavenumber $k \quad$ filter wavenumber $k$

FIG. 7. Contributions of individual components (deviatoric kinetic SGS stress, $\tau_{i j}^{\mathrm{u} *} \widetilde{\mathcal{S}}_{i j}$, and deviatoric magnetic SGS stress, $\tau_{i j}^{\mathrm{b} *} \widetilde{\mathcal{S}}_{i j}$, kinetic SGS pressure, $1 / 3 \tau_{k k}^{\mathrm{u}} \mathcal{S}_{k k}$, and magnetic SGS pressure, $1 / 6 \tau_{k k}^{\mathrm{b}} \widetilde{\mathcal{S}}_{k k}$, and EMF, $\mathcal{E} \cdot \overline{\mathbf{J}}$ ) normalized to the average SGS dissipation, $\Sigma^{E}$, of supersonic simulation $7 \mathrm{~b}$ for different filter widths. The markers illustrate the median and the error bars show the minimum and maximum values over time. Each closure family is represented by the locally best performing closures, i.e., eddy-dissipation of $\mathrm{EV}^{E}-\hat{E}_{\mathrm{sgs}}^{\mathrm{u}, \mathcal{S}}-\mathrm{ED}^{W}-\hat{E}_{\mathrm{sgs}}^{\mathrm{b}, J}-\alpha-\beta-\gamma$, scale-similarity of $\mathrm{SS}^{\mathrm{u}}-\mathrm{SS}^{\mathrm{b}}-\mathrm{SS}^{\mathcal{E}}$, and the nonlinear family of $\mathrm{NL}^{\mathrm{u}}-\mathrm{NL}^{\mathrm{b}}-\mathrm{NL}^{\mathcal{E}, \rho}$.

of forward to inverse energy transfer (not shown). While the forward transfer mediated by $\tau_{i j}^{\mathrm{b} *} \widetilde{\mathcal{S}}_{i j}$ is $\approx 30$ times stronger than the inverse transfer at $k=4$, it is only $\approx 6$ times stronger at $k=64$. At the same time, the EMF ratio remains constant at a factor $\approx 8$. Two scenarios (or more likely an unbalanced combination thereof) could potentially explain this situation: either the existence of an inverse cascade coupled to direct forward transfer or direct inverse transfer coupled with a forward cascade. On the one hand, a cascade typically transfers energy from one scale to the next smaller (or larger) scale resulting in a constant flux with varying filter width. On the other hand, direct transfer allows exchange of energy between scales with arbitrary separation and thus the flux may vary with varying filter width. Although a more detailed study, e.g., by a shell-to-shell energy transfer analysis, would allow a better interpretation, it is not required for the following closure discussion and we leave it as subject to future work.

Before analyzing the predicted contributions by the different closure families, it should be noted that the coefficients from the fits have been used to calculate the resulting dissipation values. Allowing all coefficients to vary freely and optimizing for average SGS dissipation would allow each closure to exactly match the reference data and, in turn, render this analysis meaningless. In general, all closure families behave similar with respect to the total dissipation. At large scales, they underestimate the reference data by $\approx 50 \%$ (eddy-dissipation and scale-similarity) and $\approx 40 \%$ (nonlinear), while improving towards the smallest scales reaching $\approx 75 \%$ (ED), $\approx 90 \%$ (SS), and $\approx 95 \%$ (NL) agreement. This is seen to be due to the successful capture of the EMF related contribution and failing to represent the deviatoric magnetic stress dynamics at varying filter scale. In other words, all closures predict too much net inverse energy transfer to the largest scales. Another important observation concerns the overall inverse energy transfer by the magnetic SGS pressure of the eddydiffusivity closure. Given that the eddy-viscosity and eddyresistivity closures cannot provide inverse energy transfer by construction, and that the eddy-diffusivity closure itself exhibits the overall poorest correlation as shown in subsections IV A and IV B, the SGS pressures are the only channels left for inverse transfer in this closure set. Thus, in the process of matching the inverse transfer that is present in the reference data, an over-compensation in the SGS energies takes place.

\section{Structural analysis: Topology}

We begin our structural analysis with the comparison of the deviatoric stress tensor topology. Figure 8 illustrates the amount of tube-like structures in our simulations. The only other possibility for $\tau^{\mathrm{u} *}, \tau^{\mathrm{b} *}$, and $\tau^{*}$ are sheet-like structures. Analyzing the kinetic $\tau^{\mathrm{u} *}$ and magnetic $\tau^{\mathrm{b} *}$ tensors individually we have $\approx 88 \%$ tube-like structures and $\approx 12 \%$ sheetlike structures in the data independent of tensor and sonic Mach number $M_{\mathrm{s}}$. Furthermore, there are almost no temporal variations within each simulation - the error bars indicating the minimum and maximum are within the markers. The scale-similarity closures $\mathrm{SS}^{\mathrm{u}}$ and $\mathrm{SS}^{\mathrm{b}}$ match these topologies very closely with differences of only $\approx 1 \%$. The nonlinear closures $\mathrm{NL}^{\mathrm{u}}$ and $\mathrm{NL}^{\mathrm{b}}$ are closely following the data topology as well, even though they slightly overestimate the

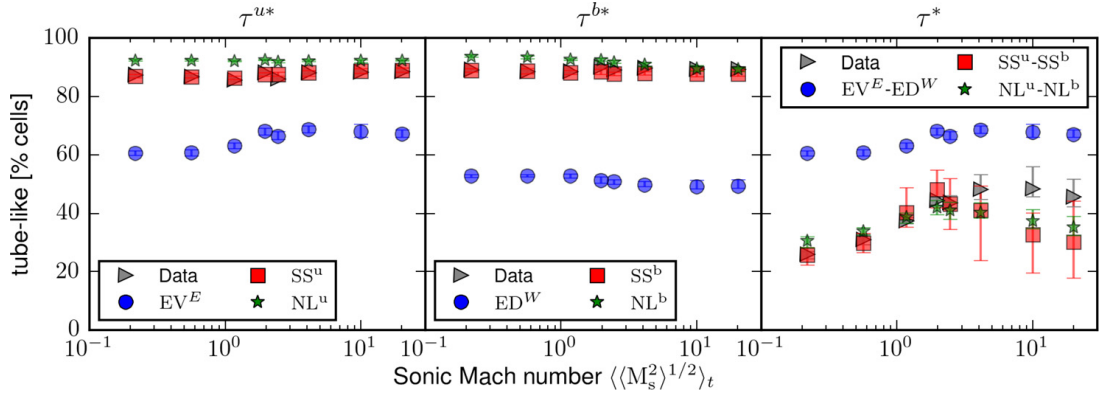

FIG. 8. Topology of deviatoric stress tensors by mean percentage of tubelike structures over all snapshots of each simulation (1, 2a, 4, 6, 7a, 11, 12, and 13, see Table I). The remaining structures are sheet-like. The error bars indicate the minimum and maximum value over time for each simulation. 


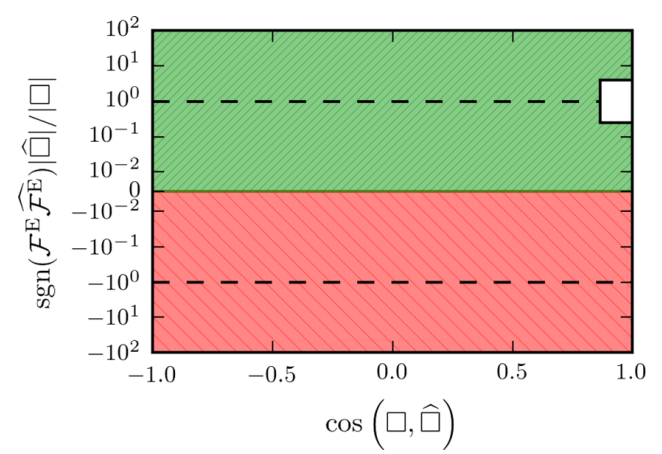

FIG. 9. Illustration of magnitude-alignment 2D-histograms (see Figure 10). The $\mathrm{x}$-axis shows the alignment between closure vector and reference vector. Relative closure magnitudes are given on the y-axis with the dashed (- -) lines indicating identical closure and reference magnitude. The upper half (green "/" hatching) indicates equal direction in energy cascade, i.e., same sign of $\mathcal{F}^{E}$ and $\widehat{\mathcal{F}^{E}}$, whereas the lower half (red " " hatching) corresponds to opposed directions. The white box illustrates the area of optimal performance: alignment is within $30^{\circ}$, relative magnitude within a factor of 4 , and identical flux sign.

amount of tube-like structure by $\approx 3 \%$ in general. Eddyviscosity $\mathrm{EV}^{E}$ and eddy-diffusivity $\mathrm{ED}^{W}$ closures on the other hand are not able to match the flow topology. While $\mathrm{EV}^{E}$ is able to reproduce at least the correct tendency with dominating tube structures $(65 \%), \mathrm{ED}^{W}$ produces an equal share of tube and sheet structures.

Interestingly, the topological configuration changes dramatically when analyzing the deviatoric tensor $\tau^{*}=$ $\tau^{\mathrm{u} *}-\tau^{\mathrm{b} *}$ as a whole. The dominant, $\mathbf{M}_{\mathrm{s}}$-independent tubelike topology vanishes, and sheet configurations become dominant in the subsonic regime. In the supersonic regime, tube- and sheet-like configurations are equally present with some $(<10 \%)$ temporal variation. Again, scale-similarity and nonlinear closures are able to follow the trend more closely than eddy-dissipation type closures. $\mathrm{EV}^{E}-\mathrm{ED}^{W}$ exhibits the same behavior as $\mathrm{EV}^{E}$ alone and provides mainly tube-like topology. The scale-similarity closure correctly captures the topology in the subsonic regime with negligible temporal variations. However, in the supersonic regime, the amount of sheet-like structures is overestimated by $15 \%$ on average and there are temporal variations of up to $14 \%$. In contrast to this, the nonlinear closure shows less variations $(<4 \%)$. However, it also overestimates sheet-like structure in the supersonic regime, but by only $10 \%$. Overall these results are in line with the original closure approaches-functional versus structural. The functional eddy-dissipation closures do not perform well in this structural test, whereas both structural closure families are capable of capturing the data topology.

\section{E. Structural analysis: Alignment and magnitude}

In order to asses how the different closures perform as vectors in the equations, i.e., $\nabla \cdot \hat{\tau}$ and $\nabla \times \hat{\mathcal{E}}$, we compare their magnitude and alignment with the reference data. Figure 9 is an explanatory sketch of the 2D-histograms we use for the analysis. The relative vector magnitude, e.g., $\left|\nabla \cdot \hat{\tau}_{i j}^{\mathrm{u} *}\right| /\left|\nabla \cdot \tau^{\mathrm{u} *}\right|$, is plotted against the angle between closure and exact solution, e.g., $\cos \left(\nabla \cdot \hat{\tau}_{i j}^{\mathrm{u}}, \nabla \cdot \tau^{\mathrm{u} *}\right)$. Furthermore, we use the sign of the product of closure flux $\widehat{\mathcal{F}^{E}}$ and reference flux $\mathcal{F}^{E}$ to split the histogram in two halves. A positive sign corresponds to the correct direction of the cascade, while a negative one indicates opposite direction. We choose this kind of presentation as it illustrates several independent measures for single-coefficient closures. First, the magnitude is a direct result of the free coefficient value that is determined by the fitting process. Second, the sign of the fluxes is determined in conjunction with a resolved flow quantity, e.g., $\widetilde{\boldsymbol{u}}$ for $\mathcal{F}^{E}=\widetilde{\boldsymbol{u}} \cdot(\nabla \cdot \tau)$, see (46), and is independent of the coefficient magnitude. Third, the angle is given by the SGS terms alone and is also independent of the coefficient magnitude. We define a region of optimal performance in order to make quantitative statements. Within this region, the relative magnitude does not deviate by more than a factor of 4 , the angle between closure and data is $<30^{\circ}$, and both fluxes $\left(\widehat{\mathcal{F}^{E}}\right.$ and $\left.\mathcal{F}^{E}\right)$ have identical sign. We use the results of the energy flux fits $\mathcal{F}^{E}$ in this subsection. Nevertheless, we also verified that the conclusions similarly apply to the other flux fits $\Sigma^{E}, \Sigma^{W}$, and $\mathcal{F}^{W}$.

Figure 10 illustrates the resulting 2D-histograms for the best performing closures in a snapshot of the supersonic simulation 7a at $t=4 T$, which has randomly been chosen for illustration purposes. The deviatoric SGS Reynolds stress $\hat{\tau}_{i j}^{\mathrm{u} *}$ closures $\mathrm{EV}^{E}, \mathrm{SS}^{\mathrm{u}}$, and $\mathrm{NL}^{\mathrm{u}}$ are shown in Figure 10(a). In general, the magnitude predicted by $\mathrm{EV}^{E}$ and $\mathrm{SS}^{\mathrm{u}}$ is too small. Furthermore, the angle between closure and data is almost randomly distributed with a slight tendency of alignment, which is more pronounced for $\mathrm{SS}^{\mathrm{u}}$. In contrast to this, $\mathrm{NL}^{\mathrm{u}}$ exhibits a clear peak at exact alignment and equal magnitude.

Over all simulations $49_{-4}^{+10} \%$ (median and bounds giving the maximum and minimum) of the cells within the simulation cube are within the region of optimal performance for $\mathrm{NL}^{\mathrm{u}}$ and $81_{-2}^{+3} \%$ have the correct sign of $\mathcal{F}^{E}$. SS ${ }^{\mathrm{u}}$ has still $66_{-2}^{+2} \%$ cells with the correct sign and $14_{-3}^{+4} \%$ in the optimal region, whereas $\mathrm{EV}^{E}$ performs worst with $5_{-4}^{+4} \%$ in the optimal region and only $58_{-2}^{+2} \%$ with equal sign.

Figure 10(b) illustrates the deviatoric SGS Maxwell closures $\mathrm{NL}^{\mathrm{b}}, \mathrm{SS}^{\mathrm{b}}$, and $\mathrm{ED}^{W}$ for the same snapshot. Overall, the nonlinear and scale-similarity closure behave very similar to their kinetic counterparts with $61_{-12}^{+13} \%$ optimal region and $84_{-5}^{+5} \%$ correct sign for $\mathrm{NL}^{\mathrm{b}}$, and $27_{-8}^{+5} \%$ optimal region and $71_{-2}^{+3} \%$ correct sign for $\mathrm{SS}^{\mathrm{b}}$, respectively. The weak performance of eddy-diffusivity closures described in subsections IV A-IV D is also apparent here. The magnitude of $\mathrm{ED}^{W}$ is typically too small by more than a factor of 10 . This comes as no surprise as it is determined by the free coefficient. Given that $\mathcal{F}^{E}$ and $\widehat{\mathcal{F}^{E}}$ have matching signs only in $52_{-1}^{+1} \%$ of the cells, which corresponds to random behavior, the fitting process favors a closure coefficient close to 0 . In addition to this, the distribution of the angle between closure and data, which is independent of the fitting procedure, is completely random and $<1 \%$ are in the optimal region.

Finally, the EMF closures $\alpha-\beta-\gamma, \mathrm{SS}^{\mathcal{E}}$, and $\mathrm{NL}^{\mathcal{E}, \rho}$ are depicted in Figure 10(c) for the same snapshot. Here, the 

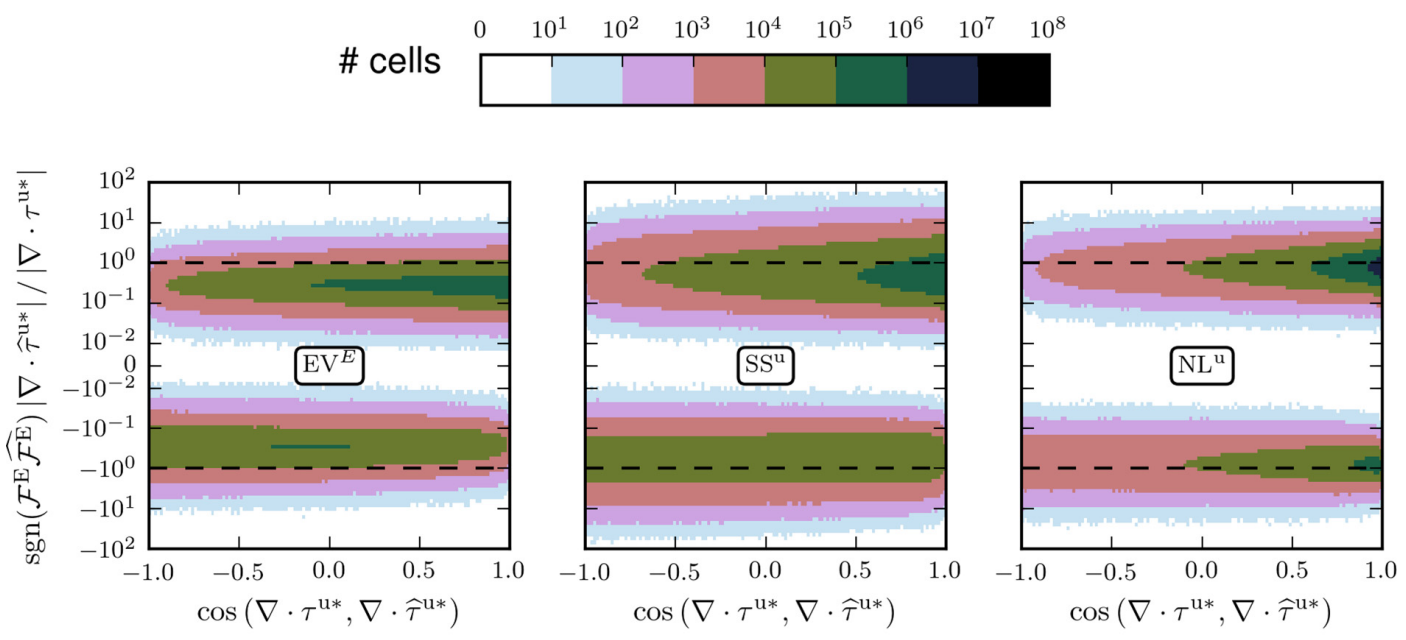

(a) Deviatoric SGS Reynolds stress closures $\widehat{\tau}_{i j}^{\mathrm{u} *}$
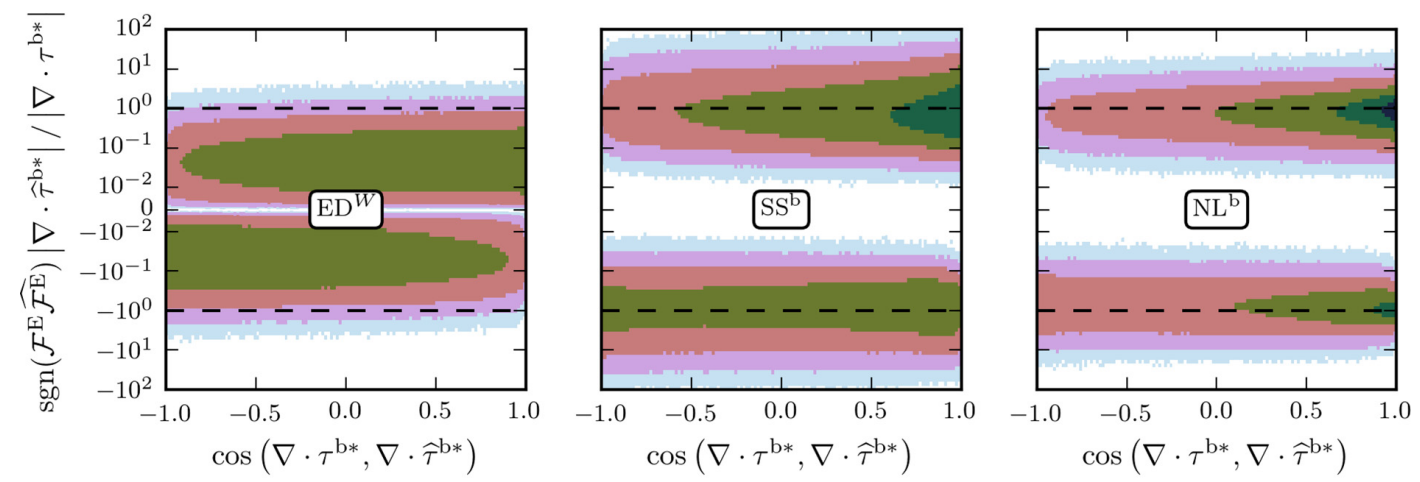

(b) Deviatoric SGS Maxwell stress closures $\widehat{\tau}_{i j}^{\mathrm{b} *}$
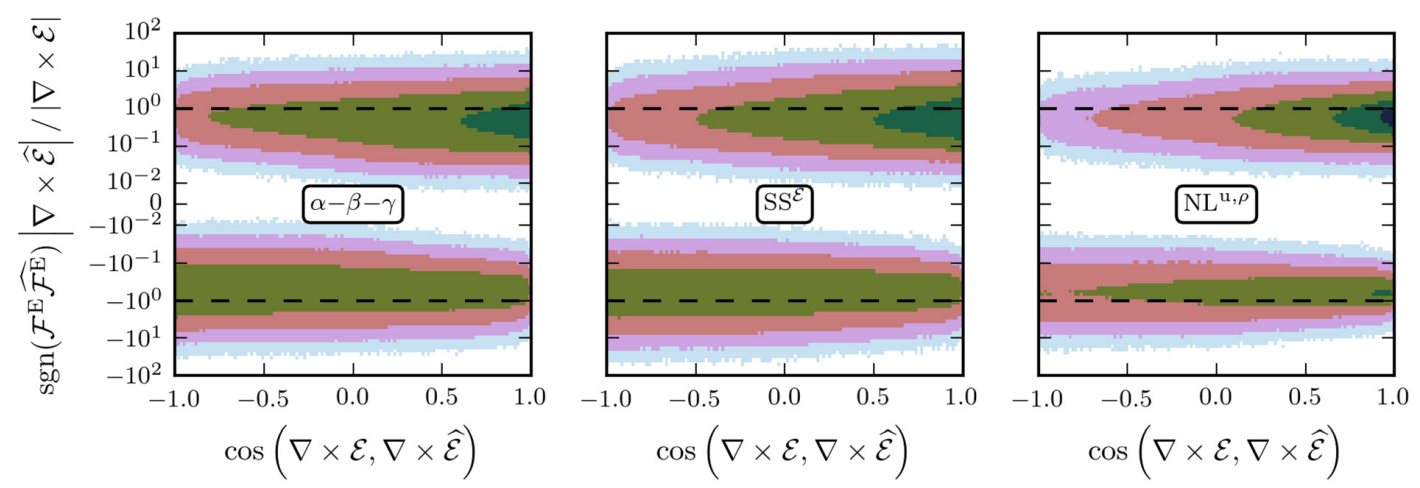

(c) Electromotive force closures $\widehat{\mathcal{E}}$

FIG. 10. Two dimensional histograms showing the distribution of relative closure vector magnitude, i.e., $\operatorname{sgn}\left(\mathcal{F}^{E} \hat{\mathcal{F}}^{E}\right)\left|\nabla \cdot \hat{\tau}^{\mathrm{u} *}\right| /\left|\nabla \cdot \tau^{\mathrm{u} *}\right|$, versus alignment, i.e., $\cos \left(\nabla \cdot \hat{\tau}^{\mathrm{u} *}, \nabla \cdot \tau^{\mathrm{u} *}\right)$, between closure and data vector. The additional signum, sgn, function on the y-axis is used to indicate flux alignment, i.e., whether data flux $\mathcal{F}^{E}$ and the flux predicted by the closure $\widehat{\mathcal{F}^{E}}$ have identical sign. Dashed lines in each plot illustrate identical closure and data vector magnitudes. The data are taken from a single snapshot at $t=4 T$ of supersonic simulation 7a filtered at $k=16$.

performance of the eddy-dissipation family closure $\alpha-\beta-\gamma$ is best compared to the other terms. Overall, $13_{-4}^{+4} \%$ cells are within the optimal region and $61_{-4}^{+5} \%$ have the correct sign. $\mathrm{SS}^{\mathcal{E}}$ performs slightly better with $19_{-7}^{+4} \%$ and $66_{-5}^{+2} \%$, respectively. In both cases, the closure vector is more likely to be aligned with the data vector even though it is not as pronounced as for the $\mathrm{NL}^{\mathcal{E}, \rho}$ closure. For the nonlinear closure $53_{-29}^{+6} \%$ are within the optimal region whereby the lower 
limit stems from the highly supersonic simulations 12 and 13. Nevertheless, $\mathrm{NL}^{\mathcal{E}, \rho}$ produces the correct flux sign in the majority of cells $\left(80_{-8}^{+3} \%\right)$ and the variation is less extensive.

The general trend that nonlinear closures are performing best, followed by scale-similarity closures and eventually eddy-dissipation closures is again visible for all terms, $\tau^{\mathrm{u} *}, \tau^{\mathrm{b} *}$, and $\mathcal{E}$.

\section{CONCLUSIONS AND OUTLOOK}

In this paper, we systematically conducted a priori tests of different subgrid-scale closures in the realm of compressible magnetohydrodynamics. Over a large parameter space of 15 simulations of forced, homogeneous, isotropic turbulence with sonic Mach numbers ranging from $\mathrm{M}_{\mathrm{s}}=0.2$ to 20 we were able to show that closures of the proposed nonlinear type outperform traditional closures of eddydissipation and scale-similarity type in every single test. The main feature of the nonlinear closures is that they require no assumptions about the nature of the flow or turbulence, and, therefore, are able to capture anisotropic effects and support up- and down-scale energy transfer. In contrast, the scale-similarity and eddy-dissipation type closures assume some universal behavior of turbulence. The $a$ priori tests included the correlation between closure and explicitly filtered reference data for quantities such as the turbulent energy $\Sigma^{E}$ and cross-helicity $\Sigma^{W}$ cascades, and total turbulent energy $\mathcal{F}^{E}$ and cross-helicity $\mathcal{F}^{W}$ fluxes. The turbulent energy cascade flux has also been used to analyze the average SGS dissipation. Additionally, we also evaluated the distribution of topological structures for the SGS Reynolds and Maxwell stress tensors and their alignment with respect to the reference data in physical space. Moreover, we verified that our conclusions are not sensitive to resolution, filter width, or filter kernel by comparing results between $512^{3}$ and $1024^{3}$ resolution simulations at filter widths of $k=4,8,16,32$, and 64 with box kernel and a Gaussian kernel. Finally, we were able to verify that the free coefficients of the basic nonlinear closures are very close to unity as expected from the analytic derivation.

Overall, we conclude that the eddy-dissipation family including the popular Smagorinsky closure has only a limited range of applicability, e.g., in situations with dominantly supersonic turbulence and in situations where local flow features are less important. Closures of the scale-similarity family or the nonlinear family can be applied in much more diverse situations, e.g., where anisotropic features or upscale energy transfer are required. However, there is still room for improvement as the net up-scale transfer via the SGS Maxwell stress is overestimated. Furthermore, the scale-similarity closures should be handled with care as their performance varies strongly with reference quantity and sonic Mach number. The basic nonlinear closures, $\mathrm{NL}^{\mathrm{u}}, \mathrm{NL}^{\mathrm{b}}$ and $\mathrm{NL}^{\mathcal{E}, \rho}$, on the other hand perform well across the entire tested parameter space and are able to reproduce local flow features.

This encourages the application of the basic nonlinear closures as a zero-coefficient SGS model in large-eddy simulations of compressible MHD. These simulations would benefit from the additional physics provided by the SGS model. Promising processes for such LES are turbulent magnetic reconnection ${ }^{2}$ or the turbulent dynamo, ${ }^{3}$ for example, in star-forming magnetized clouds ${ }^{49}$ or even in galaxies ${ }^{50}$ and clusters.

\section{ACKNOWLEDGMENTS}

The authors would like to thank C. Federrath for providing the FLASHv4 simulations. P.G. acknowledges financial support by the International Max Planck Research School for Solar System Science at the University of Göttingen. D.V. acknowledges research funding by the Deutsche Forschungsgemeinschaft (DFG) under Grant No. SFB 963/1, project A15, and the Max Planck Institute for Dynamics and Self-Organization. D.R.G.S. thanks for funding through Fondecyt regular (project code 1161247) and through the "Concurso Proyectos Internacionales de Investigación, Convocatoria 2015" (project code PII20150171). The Enzo simulations were performed and analyzed with the HLRN-III facilities of the North-German Supercomputing Alliance under Grant No. nip00037.

${ }^{1}$ C. M. Cooper, J. Wallace, M. Brookhart, M. Clark, C. Collins, W. X. Ding, K. Flanagan, I. Khalzov, Y. Li, J. Milhone, M. Nornberg, P. Nonn, D. Weisberg, D. G. Whyte, E. Zweibel, and C. B. Forest, Phys. Plasmas 21, 013505 (2014).

${ }^{2}$ J. S. Oishi, M.-M. Mac Low, D. C. Collins, and M. Tamura, Astrophys. J. 806, L12 (2015); e-print arXiv:1505.04653 [astro-ph.SR].

${ }^{3}$ J. Schober, D. R. G. Schleicher, C. Federrath, S. Bovino, and R. S. Klessen, Phys. Rev. E 92, 023010 (2015).

${ }^{4}$ M. L. Goldstein, D. A. Roberts, and W. H. Matthaeus, Annu. Rev. Astron. Astrophys. 33, 283 (1995).

${ }^{5}$ S. A. Balbus and J. F. Hawley, Rev. Mod. Phys. 70, 1 (1998).

${ }^{6}$ W. Schmidt, Living Rev. Comput. Astrophys. 1, 2 (2015).

${ }^{7}$ M. Miesch, W. Matthaeus, A. Brandenburg, A. Petrosyan, A. Pouquet, C.

Cambon, F. Jenko, D. Uzdensky, J. Stone, S. Tobias, J. Toomre, and M. Velli, Space Sci. Rev. 1, 1-41 (2015).

${ }^{8}$ A. A. Chernyshov, K. V. Karelsky, and A. S. Petrosyan, Phys. -Usp. 57, 421 (2014).

${ }^{9}$ D. G. Vlaykov, "Sub-grid Scale modelling of compressible magnetohydrodynamic turbulence: Derivation and a priori analysis," Ph.D. thesis (Georg-August University School of Science (GAUSS), Göttingen, 2015).

${ }^{10}$ A. Favre, Phys. Fluids 26, 2851 (1983).

${ }^{11} \mathrm{P}$. Sagaut, Large Eddy Simulation for Incompressible Flows: An Introduction, Scientific Computation (Springer, 2006).

${ }^{12} \mathrm{H}$. Lu and F. Porté-Agel, Physics of Fluids 22, 015109 (2010).

${ }^{13}$ B. Vreman, B. Geurts, and H. Kuerten, J. Fluid Mech. 339, 357 (1997).

${ }^{14} \mathrm{G}$. Balarac, J. Le Sommer, X. Meunier, and A. Vollant, Phys. Fluids 25, 075107 (2013).

${ }^{15}$ H. Braun, W. Schmidt, J. C. Niemeyer, and A. S. Almgren, Mon. Not. R. Astron. Soc. 442, 3407 (2014).

${ }^{16}$ M. A. Latif, D. R. G. Schleicher, W. Schmidt, and J. C. Niemeyer, Mon. Not. R. Astron. Soc. 436, 2989 (2013).

${ }^{17}$ K. Miki and S. Menon, Phys. Plasmas 15, 072306 (2008).

${ }^{18}$ M. L. Theobald, P. A. Fox, and S. Sofia, Phys. Plasmas 1, 3016 (1994).

${ }^{19}$ W.-C. Müller and D. Carati, Phys. Plasmas 9, 824 (2002).

${ }^{20}$ N. Yokoi, Geophys. Astrophys. Fluid Dyn. 37, 114-184 (2013).

${ }^{21}$ G. Balarac, A. G. Kosovichev, O. Brugière, A. A. Wray, and N. N. Mansour, in Proceedings of the Summer Program (Center for Turbulence Research, Stanford University/NASA, 2010), pp. 503-512.

${ }^{22}$ P. Grete, D. G. Vlaykov, W. Schmidt, D. R. G. Schleicher, and C. Federrath, New J. Phys. 17, 023070 (2015).

${ }^{23}$ D. G. Vlaykov, P. Grete, W. Schmidt, and D. R. G. Schleicher, Phys. Plasmas 23, 062316 (2016).

${ }^{24}$ J. Smagorinsky, Mon. Weather Rev. 91, 99 (1963).

${ }^{25}$ B. Vreman, B. Geurts, and H. Kuerten, J. Fluid Mech. 278, 351 (1994).

${ }^{26}$ O. Agullo, W.-C. Müller, B. Knaepen, and D. Carati, Phys. Plasmas 8, 3502 (2001). 


\section{Paper III: A nonlinear structural subgrid-scale closure for compressible MHD. II. A priori comparison on turbulence simulation data}

${ }^{27}$ F. Widmer, J. Büchner, and N. Yokoi, Phys. Plasmas 23, 042311 (2016).

${ }^{28}$ J. Bardina, J. H. Ferziger, and W. C. Reynolds, "Improved subgrid-scale models for large-eddy simulation," AIAA Paper No. 80-1357, 1980

${ }^{29}$ S. Liu, C. Meneveau, and J. Katz, J. Fluid Mech. 275, 83 (1994).

${ }^{30}$ A. Leonard, in Turbulent Diffusion in Environmental Pollution Proceedings of a Symposium held at Charlottesville, Advances in Geophysics 18, Part A, edited by F. Frenkiel and R. Munn (Elsevier, 1975), pp. 237-248.

${ }^{31}$ W. K. Yeo, "A generalized high pass/low pass averaging procedure for deriving and solving turbulent flow equations," Ph.D. thesis (The Ohio State University, 1987).

${ }^{32}$ P. R. Woodward, D. H. Porter, S. Anderson, T. Fuchs, and F. Herwig, J. Phys. Conf. Ser. 46, 370 (2006).

${ }^{33}$ W. Schmidt and C. Federrath, Astron. Astrophys. 528, A106 (2011).

${ }^{34}$ G. L. Bryan, M. L. Norman, B. W. O'Shea, T. Abel, J. H. Wise, M. J. Turk, D. R. Reynolds, D. C. Collins, P. Wang, S. W. Skillman, B. Smith, R. P. Harkness, J. Bordner, J. Hoon Kim, M. Kuhlen, H. Xu, N Goldbaum, C. Hummels, A. G. Kritsuk, E. Tasker, S. Skory, C. M Simpson, O. Hahn, J. S. Oishi, G. C. So, F. Zhao, R. Cen, Y. Li, and T. E. Collaboration, Astrophys. J. Suppl. Ser. 211, 19 (2014).

${ }^{35}$ B. Fryxell, K. Olson, P. Ricker, F. X. Timmes, M. Zingale, D. Q. Lamb, P. MacNeice, R. Rosner, J. W. Truran, and H. Tufo, Astrophys. J. Suppl. Ser. 131, 273 (2000)

${ }^{36}$ S. B. Pope, Turbulent Flows (Cambridge University Press, 2000).

${ }^{37}$ W. Schmidt, C. Federrath, M. Hupp, S. Kern, and J. C. Niemeyer, Astron. Astrophys. 494, 127 (2009).
${ }^{38}$ C. Federrath, J. Roman-Duval, R. S. Klessen, W. Schmidt, and M.-M. Mac Low, Astron. Astrophys. 512, A81 (2010).

${ }^{39}$ E. F. Toro, Riemann Solvers and Numerical Methods for Fluid Dynamics: A Practical Introduction (Springer Science \& Business Media, 2009).

${ }^{40}$ T. Miyoshi and K. Kusano, J. Comput. Phys. 208, 315 (2005).

${ }^{41}$ C. Federrath, G. Chabrier, J. Schober, R. Banerjee, R. S. Klessen, and D. R. G. Schleicher, Phys. Rev. Lett. 107, 114504 (2011).

${ }^{42}$ C. Federrath, J. Schober, S. Bovino, and D. R. G. Schleicher, Astrophys. J. 797, L19 (2014).

${ }^{43}$ K. Waagan, C. Federrath, and C. Klingenberg, J. Comput. Phys. 230, 3331 (2011).

${ }^{44}$ A. Dedner, F. Kemm, D. Kröner, C.-D. Munz, T. Schnitzer, and M. Wesenberg, J. Comput. Phys. 175, 645 (2002).

${ }^{45}$ S. Kitsionas, C. Federrath, R. S. Klessen, W. Schmidt, D. J. Price, L. J. Dursi, M. Gritschneder, S. Walch, R. Piontek, J. Kim, A.-K. Jappsen, P. Ciecielag, and M.-M. Mac Low, Astron. Astrophys. 508, 541 (2009).

${ }^{46}$ M. Newville, T. Stensitzki, D. B. Allen, and A. Ingargiola, LMFIT: NonLinear Least-Square Minimization and Curve-Fitting for Python, 2014.

${ }^{47}$ V. Dallas and A. Alexakis, Phys. Fluids 25, 105106 (2013); e-print arXiv:1304.0695.

${ }^{48}$ See supplementary material at http://dx.doi.org/10.1063/1.4954304 for detailed correlation and coefficient values split by simulation.

${ }^{49}$ M.-M. Mac Low and R. S. Klessen, Rev. Mod. Phys. 76, 125 (2004).

${ }^{50}$ R. Pakmor, F. Marinacci, and V. Springel, Astrophys. J. Lett. 783, L20 (2014) 


\section{Paper IV: Comparative statistics of selected subgrid-scale models in large eddy simulations of decaying, supersonic MHD turbulence}

The content of this chapter is a draft by Philipp Grete, Dimitar G. Vlaykov, Wolfram Schmidt, and Dominik R. G. Schleicher. It has been submitted to a peer-reviewed journal and is currently under review.

The research was jointly designed and planned by all authors. PG implemented the models and the filtering framework in Enzo. All simulations were run and analyzed by PG. The text was written by PG in constant exchange with the coauthors. 


\title{
5 Paper IV: Comparative statistics of selected subgrid-scale models in large eddy simulations of decaying, supersonic MHD turbulence
}

\section{Comparative statistics of selected subgrid-scale models in large eddy simulations of decaying, supersonic MHD turbulence}

\author{
Philipp Grete* \\ Max-Planck-Institut für Sonnensystemforschung, Justus-von-Liebig-Weg 3, D-37077 Göttingen, Germany and \\ Institut für Astrophysik, Universität Göttingen, Friedrich-Hund-Platz 1, D-37077 Göttingen, Germany \\ Dimitar G Vlaykov \\ Max-Planck-Institut für Dynamik und Selbstorganisation, Am Faßberg 17, D-37077 Göttingen, Germany
}

Wolfram Schmidt

Hamburger Sternwarte, Universität Hamburg, Gojenbergsweg 112, D-21029 Hamburg, Germany

Dominik R G Schleicher

Departamento de Astronomía, Facultad Ciencias Físicas y Matemáticas,

Universidad de Concepción, Av. Esteban Iturra s/n Barrio Universitario, Casilla 160-C, Chile

(Dated: July 25, 2016)

Large eddy simulations (LES) are a powerful tool in understanding processes that are inaccessible by direct simulations due to their complexity, for example, in the highly turbulent regime. However, their accuracy and success depends on a proper subgrid-scale (SGS) model that accounts for the unresolved scales in the simulation. We evaluate the applicability of two traditional SGS models, namely the eddy-viscosity (EV) and the scale-similarity (SS) model, and one recently proposed nonlinear (NL) SGS model in the realm of compressible MHD turbulence. Using 209 simulations of decaying, supersonic (initial sonic Mach number $\mathrm{M}_{\mathrm{s}} \approx 3$ ) MHD turbulence with a shock-capturing scheme and varying resolution, SGS model and filter, we analyze the ensemble statistics of kinetic and magnetic energy spectra and structure functions. Furthermore, we compare the temporal evolution of lower and higher order statistical moments of the spatial distributions of kinetic and magnetic energy, vorticity, current density, and dilatation magnitudes. We find no statistical influence on the evolution of the flow by any model if grid-scale quantities are used to calculate SGS contributions. In addition, the SS models, which employ an explicit filter, have no impact in general. On the contrary, both EV and NL models change the statistics if an explicit filter is used. For example, they slightly increase the dissipation on the smallest scales. We demonstrate that the nonlinear model improves higher order statistics already with a small explicit filter, i.e. a three-point stencil. The results of e.g. the structure functions or the skewness and kurtosis of the current density distribution are closer to the ones obtained from simulations at higher resolution. In addition, no additional regularization to stabilize the model is required. We conclude that the nonlinear model with a small explicit filter is suitable for application in more complex scenarios when higher order statistics are important.

\section{INTRODUCTION}

Magnetohydrodynamic (MHD) turbulence is observed in many different processes and on many different scales, for example, in astrophysics, in magnetized accretion disks [1], stellar winds [2], galaxies and galaxy mergers [3], or more generally in processes like magnetic reconnection [4] and the turbulent amplification of magnetic fields [5]. Moreover, experiments on Earth also aim at a better understanding of e.g. flow-driven MHD instabilities [6]. However, the full multi-dimensional dynamics are only rarely accessible in these observations and experiments. For this reason, simulations are frequently used as a third, complementary approach or to support the design of experiments [7].

Unfortunately, direct simulations with realistic parameters are often still not possible despite the ever increas- ing availability and performance of large computing clusters, and the advances in numerical methods. In these cases, large eddy simulations (LES) have been employed successfully in the past, however, mostly in the realm of (incompressible) hydrodynamics, see e.g. [8] for a general introduction and [9] for an astrophysics related review. In LES only the largest eddies, which correspond to motions on large and intermediate scales, are simulated directly. The smallest scales, which are either not represented or unresolved in these simulations, are reintroduced by the means of a subgrid-scale (SGS) model. Formally, this is equivalent to applying a low-pass filter to the ideal compressible MHD equations resulting in expressions of the

* grete@mps.mpg.de 


\section{Paper IV: Comparative statistics of selected subgrid-scale models in large eddy simulations of decaying, supersonic MHD turbulence}

form $[10]$

$$
\begin{array}{r}
\frac{\partial \bar{\rho}}{\partial t}+\nabla \cdot(\bar{\rho} \widetilde{\boldsymbol{u}})=0, \\
\frac{\partial \bar{\rho} \widetilde{\boldsymbol{u}}}{\partial t}+\nabla \cdot(\bar{\rho} \widetilde{\boldsymbol{u}} \otimes \widetilde{\boldsymbol{u}}-\overline{\boldsymbol{B}} \otimes \overline{\boldsymbol{B}})+\nabla\left(\bar{P}+\frac{\bar{B}^{2}}{2}\right) \\
=-\nabla \cdot \tau, \\
\frac{\partial \overline{\boldsymbol{B}}}{\partial t}-\nabla \times(\widetilde{\boldsymbol{u}} \times \overline{\boldsymbol{B}})=\nabla \times \mathcal{E},
\end{array}
$$

for a static, homogeneous and isotropic filter under periodic boundary conditions. The filtered primary quantities, i.e. the density $\bar{\rho}$, velocity $\widetilde{\boldsymbol{u}}$, magnetic field $\overline{\boldsymbol{B}}$ (incorporating $1 / \sqrt{4 \pi}$ ) and pressure $\bar{P}$ are considered resolved in LES. Normal filtering is denoted by an overbar $\bar{\square}$ whereas mass-weighted, Favre [11] filtering is denoted by a tilde $\widetilde{\square}=\overline{\rho \square} \bar{\square}$.

Assuming an isothermal equation of state $(\bar{P} \propto \bar{\rho})$, all interactions between resolved and unresolved scales and among unresolved scales themselves are captured by the two new terms in the equations. The turbulent stress tensor is given by

$$
\tau_{i j}=\tau_{i j}^{\mathrm{u}}-\tau_{i j}^{\mathrm{b}}+\left(\overline{B^{2}}-\bar{B}^{2}\right) \frac{\delta_{i j}}{2},
$$

and can be decomposed into the turbulent (or SGS) magnetic pressure (last term), SGS Reynolds stress $\tau_{i j}^{\mathrm{u}}$ and SGS Maxwell stress $\tau_{i j}^{\mathrm{b}}$ with

$$
\tau_{i j}^{\mathrm{u}} \equiv \bar{\rho}\left(\widetilde{u_{i} u_{j}}-\widetilde{u}_{i} \widetilde{u}_{j}\right) \text { and } \tau_{i j}^{\mathrm{b}} \equiv\left(\overline{B_{i} B_{j}}-\bar{B}_{i} \bar{B}_{j}\right)
$$

The second new term is the turbulent electromotive force (EMF):

$$
\mathcal{E}=\overline{\boldsymbol{u} \times \boldsymbol{B}}-\widetilde{\boldsymbol{u}} \times \overline{\boldsymbol{B}} .
$$

Moreover, the total filtered energy is given by

$$
\bar{E}=\underbrace{\frac{1}{2} \bar{\rho} \widetilde{u}^{2}+\frac{1}{2} \bar{B}^{2}}_{\text {(resolved) }}+\underbrace{\frac{1}{2} \bar{\rho}\left(\widetilde{u^{2}}-\widetilde{u}^{2}\right)+\frac{1}{2}\left(\overline{B^{2}}-\bar{B}^{2}\right)}_{=E_{\mathrm{sgs}}^{\mathrm{u}}+E_{\mathrm{sgs}}^{\mathrm{b}} \equiv E_{\mathrm{sgs}}(\text { unresolved })}(7)
$$

where, by virtue of the identity $\tau_{i i}^{\square} \equiv 2 E_{\mathrm{sgs}}^{\square}$ (with Einstein summation), the SGS energies are given by the traces of the corresponding stress tensor.

All these SGS terms are unclosed because the mixed terms, i.e. $\widehat{u_{i} u_{j}}, \overline{B_{i} B_{j}}$ and $\overline{\boldsymbol{u} \times \boldsymbol{B}}$, are not explicitly accessible in an LES and require modeling. This modeling is the main challenge for a successful LES.

SGS models have been subject of research in hydrodynamics for many decades, e.g. in the incompressible [8], compressible [12] and astrophysical [9] regime. However, work in the realm of MHD and in particular compressible MHD is scarce, see [13] and [14] for recent reviews. Directly linked to this work are the MHD simulations of (decaying) turbulent boxes in 3D [15], in 2D [16] and in the incompressible case $[17,18]$. However, all these groups use different numerical schemes, e.g. finite-differences or (pseudo-) spectral methods, while we employ a shockcapturing finite-volume scheme. Usually, these shockcapturing methods are thought to provide an implicit SGS model as shocks are captured by locally increasing the effective numerical dissipation with the help of e.g. slope limiting. Since this procedure is part of the overall method, these simulations are also referred to as implicit LES (ILES) [19].

In the presented work, we compare the a posteriori behavior of several SGS model including a nonlinear model that explicitly captures compressibility [10]. Their performance was previously evaluated a priori [20, 21]. Here, we analyze a set of simulations of decaying homogeneous and isotropic turbulence with respect to a set of statistical quantities including energy spectra, structure functions and statistical moments of the primary fields. More details on the numerics and the implementation are given in the following section II where we also introduce the different models tested and the setup of the particular test case of decaying compressible MHD turbulence. Afterwards, in section III we present the results of different statistics such as energy spectra, evolution of statistical moments and structure function. Then we discuss these results with respect to previous work in IV and conclude in the last section $\mathrm{V}$.

\section{METHOD}

\section{A. Subgrid-scale models}

In our a priori analysis [21] we tested three different model families, eddy-dissipation, scale-similarity, and nonlinear models, with different normalizations. For each family we identified the best model with corresponding coefficients. These models are now tested a posteriori and described in the following.

a. The eddy-viscosity (EV) model has the longest tradition with roots going back even further than its formulation for LES by Smagorinsky [22]. While originally developed for the kinetic SGS stress tensor in hydrodynamics, the general idea has been transferred to MHD $[15,18]$, where the EMF closure is usually referred to as anomalous or eddy-resistivity. The names of these functional models stem from their primary feature: purely dissipative behavior analogous to e.g. molecular viscosity and resistivity. We use the following formulations

$$
\begin{aligned}
\widehat{\tau}_{i j}^{\mathrm{u}} & =-2 \bar{\rho} \nu^{\mathrm{u}} \widetilde{\mathcal{S}}_{i j}^{*}+2 / 3 \delta_{i j} \widehat{E}_{\mathrm{sgs}}^{\mathrm{u}, \mathcal{S}^{*}}, \\
\widehat{\mathcal{E}} & =-\eta_{t} \overline{\mathbf{J}},
\end{aligned}
$$

where the strengths of the eddy-viscosity $\nu^{\mathrm{u}}$ and eddyresistivity $\eta_{t}$ are given by

$$
\begin{aligned}
\nu^{\mathrm{u}} & =C_{1} \Delta \sqrt{\widehat{E}_{\mathrm{sgs}}^{\mathrm{u}, \mathcal{S}^{*}} / \bar{\rho}} \text { and } \\
\eta_{t} & =C_{2} \Delta \sqrt{\left(\widehat{E}_{\mathrm{sgs}}^{\mathrm{u}, \mathcal{S}^{*}}+\widehat{E}_{\mathrm{sgs}}^{\mathrm{b}, \mathcal{M}}\right) / \bar{\rho}} .
\end{aligned}
$$




\section{Paper IV: Comparative statistics of selected subgrid-scale models in large eddy simulations of decaying, supersonic MHD turbulence}

They are locally scaled by the SGS energies derived from realizability conditions of the SGS stresses resulting in $[20,23]$

$$
\begin{aligned}
& \widehat{E}_{\mathrm{sgs}}^{\mathrm{u}, \mathcal{S}^{*}}=C_{3} \Delta^{2} \bar{\rho}\left|\widetilde{\mathcal{S}}^{*}\right|^{2} \quad \text { and } \\
& \widehat{E}_{\mathrm{sgs}}^{\mathrm{b}, \mathcal{M}}=C_{4} \Delta^{2}|\overline{\mathcal{M}}|^{2} .
\end{aligned}
$$

In agreement with the a priori analysis [21] the coefficients are set to $C_{1}=C_{2}=0.05$ and $C_{3}=C_{4}=0.04$. Please note that the coefficient indices are different from the ones in the referenced paper due to a reduced selection of models. Furthermore, the SGS Maxwell stress is neglected as the eddy-diffusivity extension in compressible MHD was found to not match the reference data (correlations $<0.1$ ) in the a priori analysis. Therefore, the effects of the SGS Maxwell stress are modeled implicitly by the numerical scheme.

b. The scale-similarity (SS) model originates in experimental hydrodynamics where it was observed that scale-to-scale energy transfer is self-similar up to intermittency [24]. Formally, this additional scale separation can be expressed by an additional (test) filter $\square$ whose filter width is larger than the original one. The model is given by

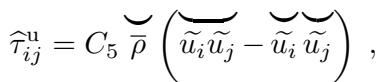

$$
\begin{aligned}
& \widehat{\tau}_{i j}^{\mathrm{b}}=C_{6}\left(\overline{\overline{B_{i} B_{j}}}-\overline{B_{i}} \overline{B_{j}}\right) \text {, } \\
& \widehat{\mathcal{E}}=C_{7}(\widetilde{\widetilde{\boldsymbol{u}} \times \overline{\boldsymbol{B}}}-\widetilde{\boldsymbol{u}} \times \overline{\boldsymbol{B}}),
\end{aligned}
$$

and mass-weighted filtering also applies to the test filter where velocity components are involved. Again, we choose the coefficients according to the a priori analysis: $C_{5}=0.67, C_{6}=0.9$ and $C_{7}=0.89$. The model allows for energy transfer down- and up-scale and, as a structural closure, aims at reproducing closely the properties of the SGS terms and not just their effects on the large scales.

c. The nonlinear (NL) model is another structural model and exhibited the highest correlations with reference data in a priori tests $[20,21]$. It can be derived from Taylor expansion of the inverse filter kernel [10, 25] and requires no further assumptions about the underlying flow features. We employ the primary compressible extension resulting in the following model:

$$
\begin{aligned}
\widehat{\tau}_{i j}^{\mathrm{u}} & =\frac{1}{12} \Delta^{2} \bar{\rho} \widetilde{u}_{i, k} \widetilde{u}_{j, k}, \\
\widehat{\tau}_{i j}^{\mathrm{b}} & =\frac{1}{12} \Delta^{2} \bar{B}_{i, k} \bar{B}_{j, k}, \\
\widehat{\mathcal{E}} & =\frac{1}{12} \Delta^{2} \varepsilon_{i j k}\left(\widetilde{u}_{j, l} \bar{B}_{k, l}-(\ln \bar{\rho})_{, l} \widetilde{u}_{j, l} \bar{B}_{k}\right) .
\end{aligned}
$$

As previously shown, this model does not require a calibration coefficient a priori [21] and the prefactor $1 / 12$ originates from the second moment of the Gaussian or box filter.
TABLE I. Filter weights for a discrete representation of the box filter based on an optimal filter approach by minimizing the residual between the analytic and discrete filter over wavenumbers below the filter width. The filter width $\bar{\Delta}$ is given in terms of grid-spacing $\Delta_{x}$

\begin{tabular}{lcccc}
\hline \hline \multirow{2}{*}{ Identifier } & filter width & \multicolumn{3}{c}{ filter weights } \\
& $\bar{\Delta}$ & $w_{i}$ & $w_{i \pm 1}$ & $w_{i \pm 2}$ \\
\hline GS & $1 \Delta_{x}$ & 1 & 0 & 0 \\
F3 & $2.711 \Delta_{x}$ & 0.4015 & 0.29925 & 0 \\
F5 & $4.7498 \Delta_{x}$ & 0.20238 & 0.22208 & 0.17673 \\
\hline \hline
\end{tabular}

\section{B. Implementation and explicit filtering}

We implemented the different models in the opensource, community-developed magnetohydrodynamic code Enzo [26]. The new terms are handled by operatorsplitting within the MUSCL-Hancock framework and evaluated with centered finite-differences. They are advanced in time together with the other fluxes by the existing second order Runge-Kutta scheme.

Furthermore, we implemented a flexible filtering framework that supports different stencils and weights in real space. In order to determine the individual weights, we construct discrete, explicit filters by minimizing the residual between analytic and discrete filter yielding so called optimal filters [27]. We optimize for wavenumbers below the filter width [28]. The resulting weights and filter widths for a symmetric one-dimensional 3-point (F3), and 5-point (F5) stencil of the box kernel are listed in table I. We construct the corresponding multi-dimensional filters of $N$-point one-dimensional stencils by

$$
\bar{\square}_{i, j, k}=\sum_{l=-N^{*}}^{N^{*}} \sum_{m=-N^{*}}^{N^{*}} \sum_{n=-N^{*}}^{N^{*}} w_{l} w_{m} w_{n} \square_{i+l, j+m, k+n},
$$

with discrete filter weights $w_{i}, N^{*}=(N-1) / 2$, and indices referring to discrete spatial locations. This translates to the sequential application of the one-dimensional filters and results in large 3-d filter stencils $\left(N^{3}\right)$, e.g. 27 points for the F3 filter and 125 points for the F5 filter. However, this construction is more accurate [28] than the alternative approach of simultaneous application. Finally, we also use the trivial grid filter (GS). In that case, the quantities are used as they are computed in the original simulation, which corresponds to a natural filter by the discretization itself.

\section{Simulations}

All our simulations are run with ENzo [26] using the HLL Riemann solver within the MUSCL-Hancock framework with second order Runge-Kutta time integration. Moreover, we close the equations of ideal MHD with a quasi-isothermal equation of state, i.e. we employ 


\section{Paper IV: Comparative statistics of selected subgrid-scale models in large eddy simulations of decaying, supersonic MHD turbulence}

an ideal equation of state with a ratio of specific heats $\kappa=1.001$.

In order to get proper initial conditions for freely decaying, compressible MHD turbulence, we first start from uniform initial conditions $\rho=1, \boldsymbol{u}=\mathbf{0}$ and $\boldsymbol{B}=(0.6325,0,0)^{\mathrm{T}}$ (in code units) corresponding to an initial plasma beta (ratio of thermal to magnetic pressure) of $\beta_{\mathrm{p}}=5$. These uniform initial conditions are then driven in a cubic box with length $L=1$ and resolution $512^{3}$ by a stochastic forcing field that evolves in space and time [29]. The forcing field has a parabolic profile between wavenumber $1<k<3$ and is centered at $k=2$. Moreover, the overall forcing amplitude is set to $V=3$ and distributed between $1 / 3$ compressive and $2 / 3$ solenoidal components. The forcing leads to statistically isotropic, homogeneous turbulence with root mean square sonic Mach number of $\mathrm{M}_{\mathrm{s}} \approx 3$ after two turnover times $T=L /(2 \mathrm{~V})$ and we follow its evolution for a total of 20T. Assuming that two different snapshots are statistically independent from each other after one turnover time, we have an ensemble of 19 different realizations (at $t=\{2,3, \ldots, 20\} T$ ) of isotropic, homogeneous turbulence, which are statistically indistinguishable. We take states from this ensemble as initial conditions for freely decaying turbulence. This later allows us to analyze ensemble statistics to better capture the intermittent nature of turbulence.

For each realization we run the following 11 simulations with different configurations - varying the resolution, models (or lack thereof) and explicit filter, namely:

- 3 implicit large-eddy simulations (ILES). Recall that in these simulations there is no explicit model $(\tau=\mathcal{E}=0)$, at resolutions of $128^{3}, 256^{3}$ and $512^{3}$.

- 3 LES with the eddy-viscosity model at a resolution of $128^{3}$ : one with no explicit filter (EV-GS); one with a filter with a three-point stencil (EV-F3); and one with a five-point stencil (EV-F5).

- 2 LES with the scale-similarity model at $128^{3} \mathrm{em}$ ploying three- (SS-F3) and five-point (SS-F5) explicit filtering (because a grid-scale scale-similarity model does not exist).

- 3 LES with the nonlinear model again at $128^{3}$ with all three different filters NL-GS, NL-F3, and NLF5.

The highest resolution $512^{3}$ ILES simulations are later used as reference runs. Comparing the results between the different ILES enables us to evaluate the pure influence of reduced resolution (and thus reduced dynamics) on the evolution of the decay. From a comparison of results between ILES and the different LES at identical resolution (here $128^{3}$ ) we can draw conclusions on the influence of the individual models on the evolution.

Each simulation follows the decay for two turnover times. We capture snapshots every $0.05 T$ resulting in 40 snapshots per simulation. Finally, we note that the initial conditions at lower resolutions $\left(128^{3}\right.$ and $\left.256^{3}\right)$ have been calculated from the initial $512^{3}$ snapshot of each realization by coarse-graining, i.e. volume-averaging over $2^{3}$ and $4^{3}$ cells, respectively. We choose this approach to minimize the differences in the initial conditions between the individual configurations of a particular realization. In addition to this, all simulations, including the LES, first decay for $0.2 T$ without model before the actual $2 T$ decay that we follow and analyze. This is done in order to obtain converged spectra at a given resolution, because the process of coarse-graining leaves excess energy at the smallest scales and the interaction between model and excess energy is unknown. Moreover, resolution dependent quantities, e.g. magnetic energy or vorticity (see next section), relax to their intrinsic value in this transientdecay.

\section{RESULTS}

\section{A. Energy spectra}

Figure 1 shows the kinetic and magnetic energy spectra initially at $t=0 T$ and after two turnover times at $t=2 T$.

Initially, there is basically no variation of the individual spectra between different configurations. However, the difference in resolution is clearly visible. The highest resolution ensemble (ILES-512) exhibits the most extended power-law range in the kinetic energy spectrum. Accordingly the wavenumber $k$ where the spectrum drops due to numerical dissipation is shifted towards larger scales with decreasing resolution (ILES-256 and ILES-128). This also confirms our approach of removing coarse-graining artifacts in the initial snapshots by the initial transientdecay. We verified that the smallest scales are statistically stationary in the following evolution. The small peak still visible around $k=2$ is due to the original characteristic driving scale of the initial forced simulation.

After two turnover times, the differences between resolutions remain the most striking feature in the spectra with respect to the wavenumbers where the spectrum wears off. At the lowest resolution, the differences between the ILES-128 and the different SGS models are subtle. There exists virtually no difference between the ILES-128 and the grid-filtered eddy-viscosity (EV-GS) and nonlinear (NL-GS) model or the scale-similarity runs (SS-F3 and SS-F5) - both in the kinetic and in the magnetic spectrum. The SGS runs of the eddy-viscosity model with explicit filtering lead to a slightly stronger dissipative behavior. This is expressed by a marginal reduction of energy on the smallest scales $k \gtrsim 30$ and more pronounced $(\approx 40 \%)$ for the larger filter width (EV-F5) than for the smaller filter width $(\approx 20 \%-\mathrm{EV}-\mathrm{F} 3)$. The nonlinear model (NL-F3 and NL-F5) seems to have a very similar dissipative behavior to the eddy-viscosity model given that the spectra coincide for the same filter. 


\section{Paper IV: Comparative statistics of selected subgrid-scale models in large eddy simulations of decaying, supersonic MHD turbulence}
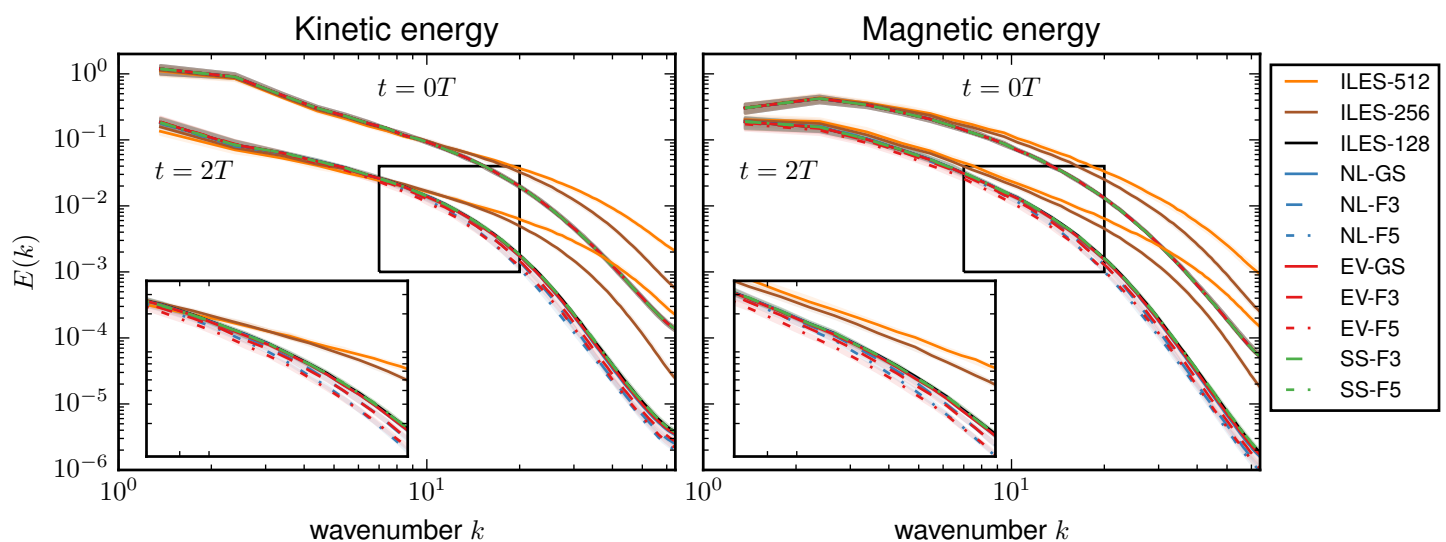

FIG. 1. Kinetic and magnetic energy spectra at initial time $t=0 T$ and after two turnover times $t=2 T$ of free decay. The lines correspond to the median over all 19 realizations and the shaded areas (if not hidden by the linewidth) show the interquartile range. The kinetic energy spectrum has been calculated based on the Fourier transform of $\sqrt{\rho} \boldsymbol{u}$. The insets show a magnification of the configurations in the intermediate wavenumber range at $t=2 T$.

\section{B. Temporal evolution of mean quantities}

The evolution over time of the spatially averaged kinetic energy, magnetic energy, vorticity magnitude, current density magnitude and dilatation magnitude is shown in figure 2. Overall, all quantities smoothly decay over the two turnover times as expected. The panels show a similar behavior of the SGS models as observed in the energy spectra. However, there are subtle differences.

The evolution of the kinetic energy, $\rho \boldsymbol{u}^{2} / 2$, is converged with respect to resolution and SGS model. In contrast to this, the magnetic energy, $\boldsymbol{B}^{2} / 2$, shows a clear separation with resolution. The decreased turbulence intensity or effective Reynolds number at lower resolutions cannot sustain the original magnetic field strength of the driven simulation conducted at higher resolution. Thus, the differences in the initial values at $t=0 T$ can be attributed to the removal of coarse-graining artifacts (here, the excess magnetic energy for a given resolution) by the transient-decay. When removing the resolution effects, e.g. by normalizing each ensemble to its initial value, all configurations but one (EV-F5) collapse to a converged evolution. The eddy-viscosity model with the largest explicit filter shows $\mathrm{a} \approx 10 \%$ decrease in magnetic energy indicating increased dissipation. However, in constrast to the energy spectra, here not only the small scales are affected by the model, but a back-reaction onto the largest scales has taken place.

The derived quantities, i.e. the vorticity magnitude $|\nabla \times \boldsymbol{u}|$, the current density magnitude $|\nabla \times \boldsymbol{B}|$ and the dilatation magnitude $|\nabla \cdot \boldsymbol{u}|$, exhibit comparable behavior. In the raw data, resolution effects dominate and resolving smaller spatial scales leads to larger values. Contrary to the evolution of the magnetic energy, this effect does not vanish when all configurations are normalized and a lower resolution results in a slightly increased decay rate. The same four SGS models (EV-F3, EV-F5, NL-F3 and NL-F5) as in the energy spectra now separate from the bulk (EV-GS, NL-GS, SS-F3 and SS-F5), which is indistinguishable from the ILES-128 ensemble. The vorticity and current magnitudes are reduced by $5 \%$ (NL-F3), $6 \%$ (EV-F3), 10\% (NL-F5) and 12\% (EV-F5) indicating very similar behavior with respect to filter width between eddy-viscosity and nonlinear model. In contrast, the dilatation magnitude is only reduced by $5 \%$ (F3) and $6 \%$ (F5) for the NL models, but by $8 \%$ (F3) and 16\% (F5) for the EV models. The latter indicates that the eddyviscosity model is more isotropic than the nonlinear one in agreement with their functional form.

\section{Higher-order statistics}

After having described the evolution of mean quantities, we now consider the temporal evolution of the higher order moments of the distributions. They provide insight into the tails of the distributions, which are crucial in the characterization and understanding of, for instance, the intermittency of turbulence. In general, the variances of all quantities (kinetic and magnetic energy, and vorticity, current and dilatation magnitudes) posses the same characteristics as their means in the previous section, i.e. an overall decay is observed with similar ensemble variations and configuration separations.

The next higher order moments we consider are the 


\section{Paper IV: Comparative statistics of selected subgrid-scale models in large eddy simulations of decaying, supersonic MHD turbulence}

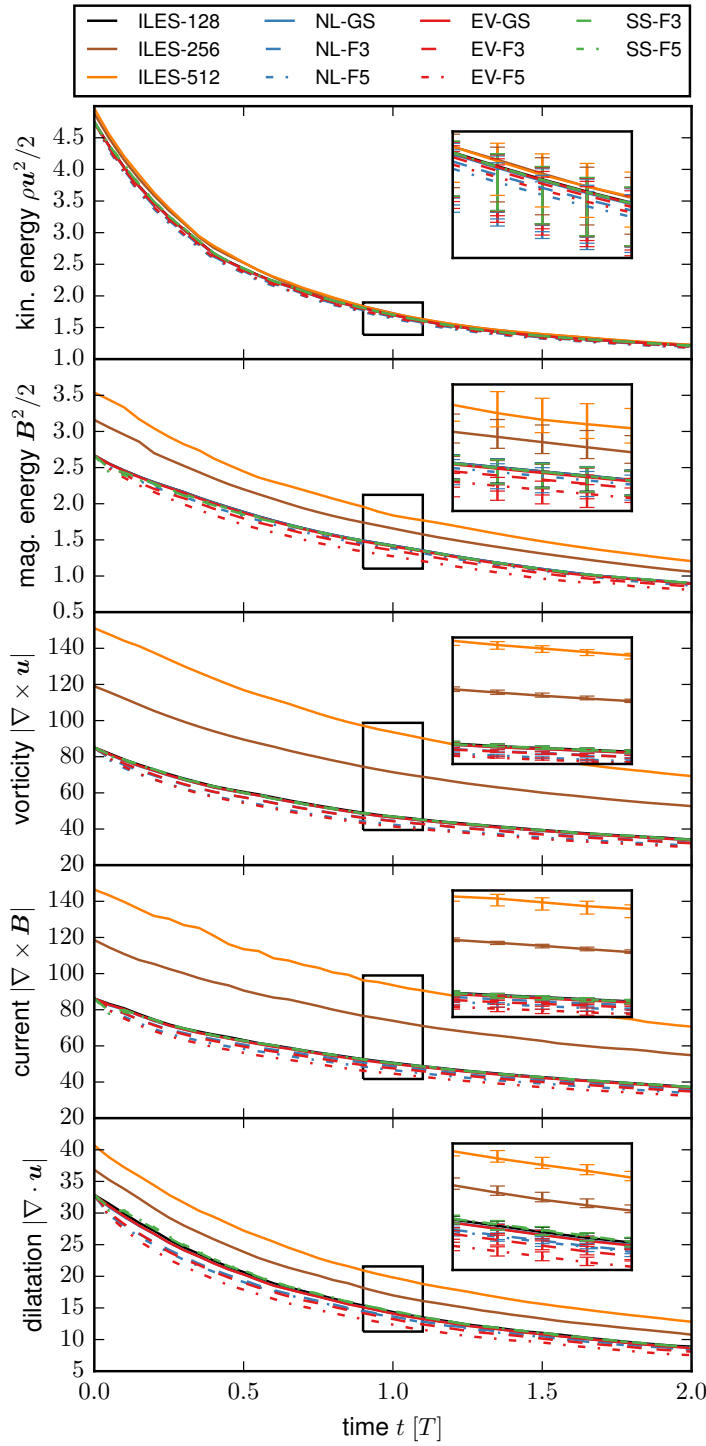

FIG. 2. Temporal evolution of the ensemble median (over 19 different realizations) of the spatial mean kinetic energy, magnetic energy, vorticity magnitude, current density magnitude and dilatation magnitude. The variations in terms of interquartile ranges are illustrated in the insets.

skewness with standard deviation $\sigma$ and the (Fisher) kurtosis

$$
\operatorname{kurt} x=\frac{\left\langle(x-\langle x\rangle)^{4}\right\rangle}{\sigma^{4}(x)}-3 .
$$

The kinetic and magnetic energy skewness and kurtosis do not discriminate between the different models as the ensemble variations for each configuration are larger than the differences between the configurations. This picture changes when looking at the higher order moments of the derived quantities. The temporal evolution of the skewness and kurtosis of the vorticity, dilatation and current magnitude are very similar (with respect to the qualitative evolution of the medians and interquartile ranges) as shown in figure 3 . Thus, we focus on the magnitude of the current density for a quantitative discussion. Firstly, both skewness and kurtosis are resolution dependent. A lower resolution increases the skewness by $\approx 5 \%$ (ILES256 vs ILES-512) and $\approx 10 \%$ (ILES-128 vs ILES-1024), and the kurtosis by $\approx 10 \%$ and $\approx 25 \%$, respectively.

All eddy-viscosity and scale-similarity models follow this trend. They evolve virtually identically to the ILES128 ensemble. Also the nonlinear model based on gridscale quantities (NL-GS) does not have a measurable impact on the results. However, the explicitly filtered nonlinear models (NL-F3 and NL-F5) clearly improve over the ensemble without model. Their evolution is consistent with the higher resolution (ILES-256) results. Moreover, the differences between using a three-point stencil and a five-point stencil are negligible indicating a converged result.

To further illustrate this we show the instantaneous probability density function (PDF) of the current magnitude at $t=1 T$ in figure 4 . The top panel (4(a)) illustrates the raw PDFs. The ensembles at different resolutions are clearly identified by an overall shift on the x-axis. This corresponds to the decrease of the mean with resolution, as described in section III B. The differences in the higher order statistics of the different configurations are already hinted at in the insets. A pure shift would equally affect the left and right hand side tails. This is observed in the PDFs of EV-F3 and EV-F5, which are both shifted to the left in comparison to the implicit LES configuration. In contrast to this, the PDFs of NL-F3 and NL-F5 only exhibit a shift in the right tail and coincide with the ILES-128 in the left tail indicating a change in the shape of the PDF.

This difference is evident in the PDFs of the normalized current in figure $4(\mathrm{~b})$, where the individual PDFs have been normalized by the respective mean values. The three features previously identified in the temporal evolution of the skewness and kurtosis, i.e. the resolution differences (ILES-128 vs ILES-256 vs ILES-512), the insensitivity of the EV and SS models, and the improvement by the nonlinear model, are clearly present. In fact, the ensemble distributions of the nonlinear model (NL-F3 and NL-F5) at a resolution of $128^{3}$ match the distribution of the implicit LES at a resolution of $256^{3}$ demonstrat- 


\section{Paper IV: Comparative statistics of selected subgrid-scale models in large eddy simulations of decaying, supersonic MHD turbulence}
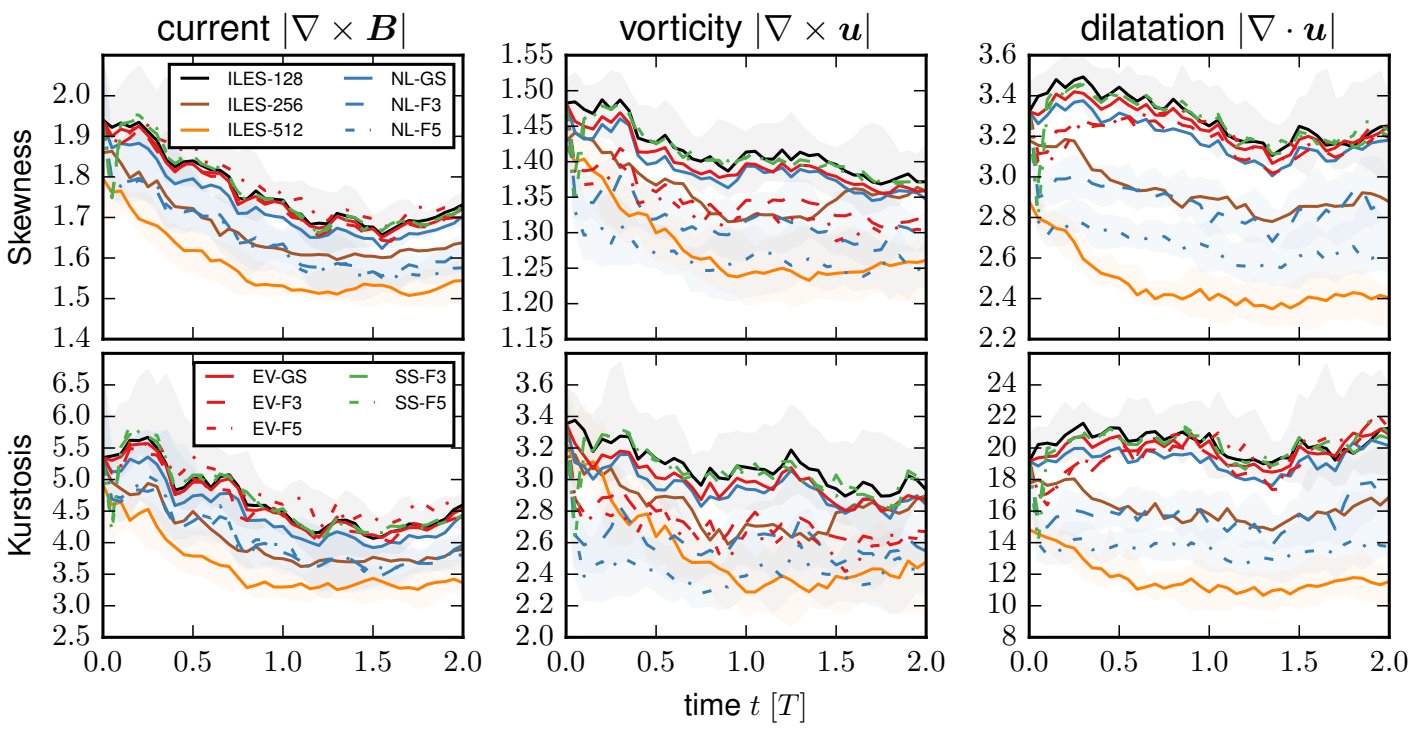

FIG. 3. Temporal evolution of the skewness and kurtosis of the distributions of current density, vorticity and dilatation magnitude. The lines indicate the median over all 19 realizations. The shaded areas correspond to the interquartile ranges (IQR). For clarity, they are only shown for ILES-128, ILES-512, NL-F3 and NL-F5 as the IQRs of similar lines are virtually identical, e.g. the lines of ILES-128, EV-GS, NL-GS, SS-F3 and SS-F5,

ing a clear enhancement. Finally, we emphasize again that the results of the current density presented in this subsection are qualitative identical to the ones obtained for the vorticity and dilatation magnitude, i.e. the explicitly filtered nonlinear models match the higher-resolution ILES.

\section{Structure functions}

In order to gain further insight into the flow we analyze the structure functions [e.g. 30]. In particular, we look at the longitudinal velocity structure functions of order $p$

$$
S_{\|}^{p}(l)=\left\langle|(\boldsymbol{u}(\boldsymbol{x}+\boldsymbol{l})-\boldsymbol{u}(\boldsymbol{x})) \cdot \boldsymbol{l} / l|^{p}\right\rangle
$$

which are given by the moments of the velocity increments along the direction of separation $\boldsymbol{l}$ assuming homogeneity and isotropy. Structure functions are related to the correlation functions and the energy spectrum. Moreover, they exhibit scaling behavior in the inertial range $S^{p}(l) \propto l^{\zeta_{p}}$ so that scaling exponents $\zeta_{p}$ can be determined. Figure 5(a) illustrates the second order longitudinal structure function $S_{\|}^{2}$ for all configurations of one arbitrary realization after one turnover time of free decay. All structure functions have been calculated based on $10^{10}$ randomly chosen pair of points. The convergence has been verified by comparing the results with the ones obtained by using twice the amount of points for one particular snapshot. Two important features can be observed. First, the structure functions of all configurations, i.e. independent of resolution and presence of an SGS model, collapse (on top of each other) on scales $\gtrsim 30 \Delta_{x}$. On smaller scales, the differences with respect to resolution are more pronounced. This is expected since the increasing numerical dissipation with decreasing resolution leads to a decrease of variations in the velocity field on the small scales. Again, the grid-filtered EV and NL LES, and the scale-similarity runs are indistinguishable from the ILES run. The increased dissipation of the EV and NL model already observed in the spectra and mean quantities is also visible here in the slightly reduced variations on the smallest scales. Second, no clear power-law range can be identified in any of the configurations which can be attributed to the limited resolution, which for these simulations indicates a too low Reynolds number.

For this reason, we make use of the concept of extended self-similarity (ESS) stating that the scaling behavior with corresponding scaling exponents can be recovered by relating structure functions to each other. While originally discovered in hydrodynamics [31], this concept works remarkably well in MHD, too. For example, in figure $5(\mathrm{~b})$ we plot $S_{\|}^{2}$ versus $S_{\|}^{3}$ (of the same snapshot as in figure 5(a)). A power-law behavior for all configurations is clearly visible. Moreover, the scaling exponents in this representations are by construction 


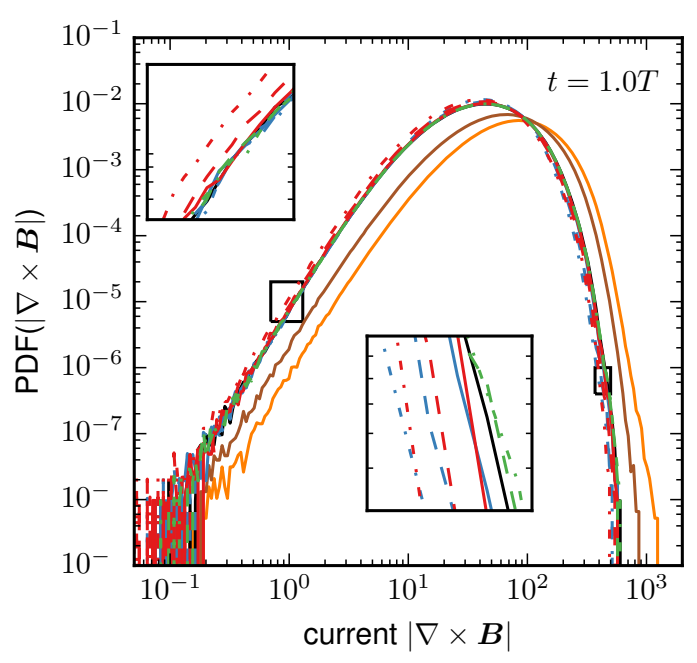

(a) Original raw data.

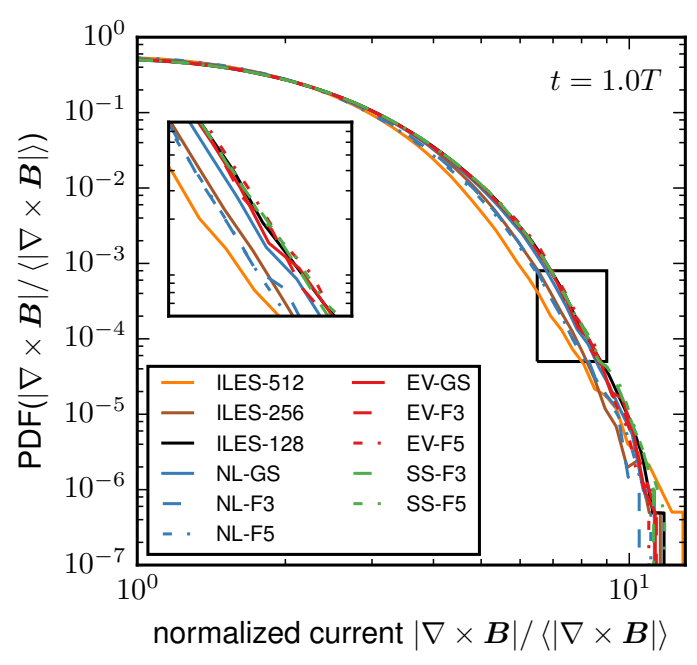

(b)Detailed illustration of right tail. Here, the current has been normalized by the respective mean value of each configuration in order to highlight resolution independent features.

FIG. 4. Probability density function of the stacked current density magnitude, i.e. each bin contains the sum over all 19 realizations, at $t=1 T$. The insets are magnifications of the indicated regions.

identical to the original ones. Thus, we determine the individual exponents in this representation by nonlinear least-square minimization using the LMFIT package [32] With these exponents, we now continue our analysis in two directions: reevaluation of the structure functions versus separation distance, and scaling behavior with increasing order $p$.

Figure 5(c) shows the median (over all 19 realiza-

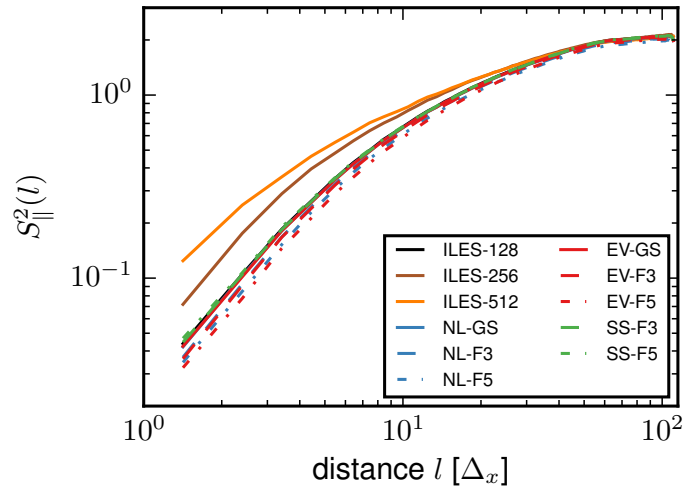

(a)Second order longitudinal structure function of one arbitrary realization.

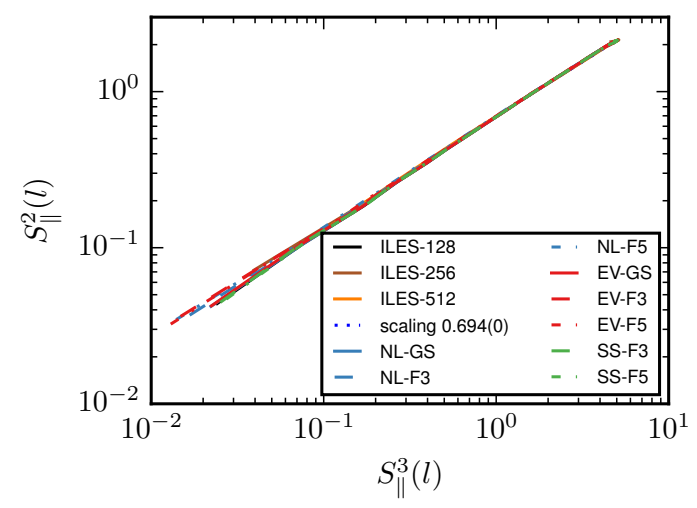

(b)Second order versus third order structure function illustrating extended self-similarity of one arbitrary realization. The best power-law fit (blue, dotted ...) to the ILES-512 simulation has an index of $0.694(0)$.

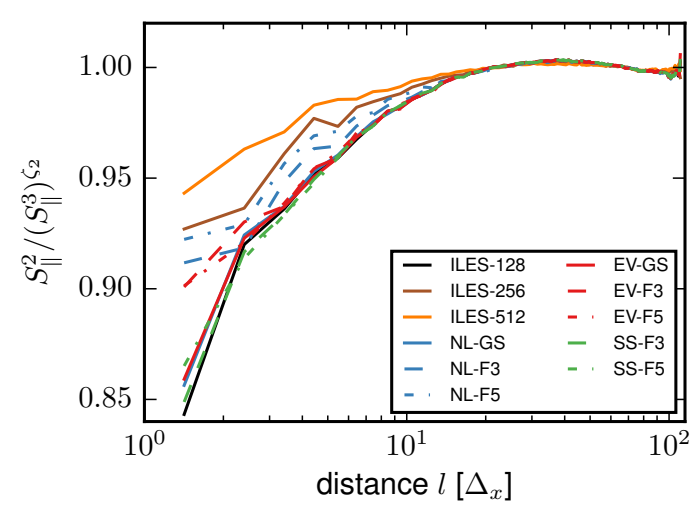

(c)Second order structure functions normalized to the third order structure function scaled by the best-fit exponent. The lines indicate the median over all 19 realizations. Variations as measured by the interquartile range are $<9 \%$.

FIG. 5. Different illustrations of second order longitudinal velocity structure functions $S_{\|}^{2}(l)$ at $t=1 T$. 


\section{Paper IV: Comparative statistics of selected subgrid-scale models in large eddy simulations of decaying, supersonic MHD turbulence}

tion) second order structure function of all configurations compensated by the corresponding third-order structure function and scaling exponent, i.e. $S_{\|}^{2} /\left(S_{\|}^{3}\right)^{\zeta_{2}}$, versus distance $l$. The plot illustrates where and to what extent the power-law scaling is found in the non-normalized data. Approximate power-law scaling is observed for all configurations on scales $\gtrsim 20 \Delta_{x}$. Below $20 \Delta_{x}$ the individual configurations start to deviate from ideal scaling. The deviations grow towards smaller scales for all configurations, however, to different degrees for different configurations. As expected, the highest resolution runs (ILES-512) exhibit the least deviation (at most $6 \%$ on the smallest scale), followed by the intermediate resolution runs (ILES-256) with $\approx 7 \%$ on the smallest scale. At the lowest resolution, the no-model (ILES-128), grid-scale filtered SGS (EV-GS and NL-GS) and scale-similarity (SSF3 and SS-F5) runs show the strongest deviation, $\approx 15 \%$. The explicitly filtered eddy-viscosity (EV-F3 and EVF5) and nonlinear models (NL-F3 and NL-F5) display an improved behavior over the other low resolution runs. While the two EV models deviate by $10 \%$, the nonlinear models deviate $8-9 \%$, reaching almost the performance of the intermediate resolution runs. Qualitatively, the same behavior observed for the structure functions of order $p=2$ is also observed for the structure functions of order $p=1$ and higher orders.

Finally, we analyze how the scaling exponents evolve with order $p$ depending on resolution and presence of an SGS model. Figure 6 illustrates the median coefficients $\zeta_{o}$ over all 19 realizations up to order $p=6$. The coefficients have been determined based on nonlinear least-square fitting and employing extended self-similarity. For higher orders the ESS does not provide robust exponents any more. The left panel of figure 6 shows the influence of decreasing resolution on the exponents. While the exponents up to $p=4$ are virtually identical, a lower resolution (ILES-128 and ILES-256) leads to a slight overestimation of $\zeta_{5}(2 \%)$ and $\zeta_{6}(3 \%)$ in comparison to ILES512. For reference, we also plot the exponents as derived by She and Leveque [33] under the assumption that the most singular dissipative structures are filaments

$$
\zeta_{p}=\frac{p}{9}+2\left(1-\left(\frac{2}{3}\right)^{p / 3}\right) .
$$

The reference run ILES-512 fits the prediction remarkably well with a deviation of only $8 \%$ at the highest order $p=6$. In general, the different SGS models and filtering procedures do not have a measurable influence on the scaling behavior. All behave like the ILES-128 yielding slightly overestimated exponents at high order, which can be attributed entirely to the low resolution.

\section{E. Computational efficiency}

Finally, we compare the additional computational costs incurred with the calculation of the different SGS
TABLE II. Computational efficiency of the different SGS models relative to the no-model run at identical resolution (ILES-128). For reference, the efficiency of the no-model run at the next higher resolution (ILES-256) is also shown. The numbers represent the mean values (at the fluid level) over three test runs of 300 cycles each. Each run used the same executable on a single machine employing 8 MPI-processes (no threading). This corresponds to a $64^{3}$ grid per process, as suggested by the ENZO documentation.

\begin{tabular}{lccc}
\hline \hline & GS & F3 & F5 \\
\hline NL & $1.175(5)$ & $1.468(5)$ & $2.411(7)$ \\
EV & $1.133(5)$ & $1.431(6)$ & $2.368(8)$ \\
SS & & $1.949(8)$ & $4.804(16)$ \\
ILES-256 & $9.242(28)^{\mathrm{a}}$ & & \\
\hline \hline
\end{tabular}

a Please note that this number only includes the time per cycle. The total computational costs are increased by another factor of $\gtrsim 2$ due to the decreased timestep at higher resolution.

models and the filtering. Given that all LES were conducted at a resolution of $128^{3}$ grid points, we compare the relative overhead over the no-model ensemble at the same resolution (ILES-128). Table II lists the mean ratios of the time per individual cycle at the fluid level, i.e. other factors such as inter-process communication are not included. The time per cycle increases for all SGS models when compared to the ILES-128 as expected. Furthermore, two general trends are visible.

First, the computational costs increase with increasing filter width. For example, the nonlinear model with gridscale quantities increases the time per cycle by a factor of $\approx 1.18$ (NL-GS). Explicit filtering introduces additional computations and is thus even more expensive, i.e. a factor of $\approx 1.47$ for NL-F3 and of $\approx 2.41$ for NL-F5, respectively. The unproportional increase in computational costs between F3 and F5 is easily explained by the unfavorable memory access in the filtering procedure. For F5 the filter is build upon a stencil involving $5^{3}=125$ points resulting in many accesses to non-contiguous memory and thus cache-misses.

Second, the eddy-viscosity and nonlinear model introduce a similar overhead, with EV being a few percent cheaper than NL, while the scale-similarity model is about twice as expensive as the other two models. The latter is attributed to the additional (comparatively expensive, explicit) filter operations. NL and EV only require filter operations on the 7 primary quantities $(\bar{\rho}, \widetilde{\boldsymbol{u}}$ and $\overline{\boldsymbol{B}})$. The scale-similarity models also needs all filtered mixed quantities $(\widetilde{u_{i} \widetilde{u}_{j}}, \overline{B_{i} B_{j}}$, and $\underbrace{}_{\widetilde{\boldsymbol{u}} \times \overline{\boldsymbol{B}}})$ which involves 15 additional filter operations in total.

Finally, we also tested how the computational costs increase for a no-model run at the next higher resolution (ILES-256). At the level of a single fluid cycle the time increases by factor of $\approx 9$. However, this does not yet take into account that the timestep is also reduced by a factor of $\gtrsim 2$ at $256^{3}$ versus $128^{3}$. Thus, the total time required to reach a certain state in the simulation 


\section{Paper IV: Comparative statistics of selected subgrid-scale models in large eddy simulations of decaying, supersonic MHD turbulence}

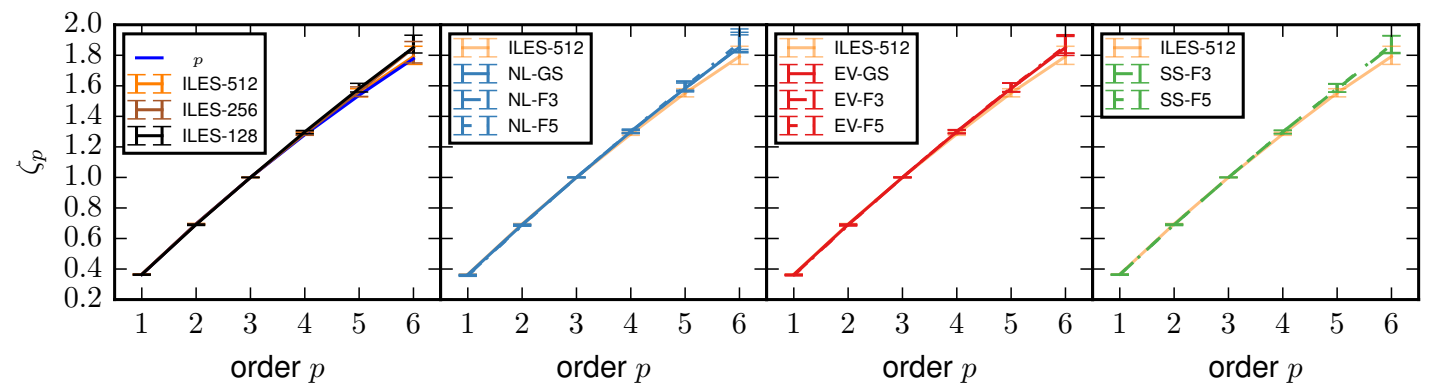

FIG. 6. Scaling exponents $\zeta_{p}$ of the longitudinal velocity structure functions of order $p$ at $t=1 T$. The lines indicate the median over all 19 realization and the errorbars illustrate the interquartile range. For reference, the theoretical scaling $\zeta_{p}^{\mathrm{SL}}$ derived by She and Leveque [33] is shown in the left panel, and the reference simulation (ILES-512) is drawn in all panels.

is effectively increased by a factor of $\gtrsim 18$.

\section{DISCUSSION}

One of the most striking results from the analysis in the last section is that models calculated from quantities at the grid-scale, i.e. EV-GS and NL-GS, and scalesimilarity models (SS-F3 and SS-F5) have no measurable impact on the statistics of the flow. The results for the grid-scale based models are in agreement with findings for finite-difference schemes [34, 35] and for shock-capturing methods [36], i.e. numerics dominate over (eddy-viscosity type) SGS models when no explicit filtering is applied. However this does not explain the results for the scalesimilarity closures, which employ an explicit filter. A possible explanation for the absence of any observable effect (apart from a very short transient behavior, e.g. in the kurtosis and skewness of the current at $t<0.1 T$ as visible in figure 3 ) is that the filter separation is still too small. Physically, this translates to the statement that the modeling assumption of self-similar turbulence is not fulfilled on these numerically strongly damped scales.

Another observation concerns the convergence with filter width for explicitly filtered eddy-viscosity (EV-F3 and EV-F5) and nonlinear (NL-F3 NL-F5) models. The energy spectra and mean quantities exhibit a small dependency on the filter width indicating increased dissipative behavior with larger $\Delta$. However, this is secondary from a practical point of view because a smaller explicit filter is desireable for two reasons. First, higher order statistics, e.g. skewness, kurtosis and normalized structure functions, show approximately converged results for F3 and F5. Thus, the improvements over the ILES-128 can already be achieved with the smaller explicit filter and the nonlinear model (NL-F3) while possessing a smaller intrinsic dissipation and being computationally more efficient than the F5 counterpart. Second, larger explicit filters in their current form are impractical for actual LES in any case.

Due to the unfavorable memory access in the filtering, the computational cost grows exponentially with increasing filter width. In addition, more and more ghost zones are required increasing the costs even further. Building multi-dimensional filters based on the simultaneous application of one dimensional ones rather than the sequential application could be a potential way out. Even though they are found to be slightly less accurate [28], their multi-dimensional stencil size is decreased dramatically, i.e. from $N^{3}$ to $3(N-1)+1$ supporting points in three dimensions with $N$ being the number of points for the 1-d filter. Alternatively, the filtering could be realized in spectral space. While the process of filtering itself is then reduced to a simple local multiplication, additional complexity independent of the filter width, is introduced by the transformations between real and spectral space.

Our current filtering framework could also be further optimized to reduce the computational overhead of the filtering. For example, cache misses would be partly avoided by using fixed, compiled-in stencils rather than dynamic ones in each cycle. Independently of this, in practice the estitamed SGS modeling overhead in a simulation is rather conservative. The total wallclock time always depends on additional factors than the time spent at the pure fluid level, most notably inter-process communication. Moreover, additional physics present in the simulation such as gravity or chemistry can reduce the relative overhead introduced by an SGS model even further.

Finally, we comment on the numerical stability of the different models. All models and filters lead to stable simulations and we did not employ any explicitly regularization. While this comes as no surprise for the eddyviscosity type models, which are only capable of transferring energy down-scale, other groups, e.g. [17, 37], typically find that regularization is required for scalesimilarity and nonlinear type models. These models also allow for up-scale energy transfer and are thus capable of seeding numerical instabilities when this inverse 


\section{Paper IV: Comparative statistics of selected subgrid-scale models in large eddy simulations of decaying, supersonic MHD turbulence}

transfer is not controlled. Most frequently, both type of models are therefore supplemented with an additional eddy-viscosity type term which successfully stabilizes the simulations. However, this only concerns (non shockcapturing) finite-volume, finite difference or (pseudo-) spectral schemes. In our case of a shock-capturing finitevolume scheme, the inherent numerical dissipation acts as an effective eddy-viscosity model (thus the term implicit LES, see e.g. [19]) and evidently provides sufficient regularization for stable simulations.

\section{CONCLUSIONS AND OUTLOOK}

In this paper we analyzed the free decay of homogeneous, isotropic, supersonic MHD turbulence with different SGS models and without explicit model on various grid resolutions. SGS models are typically introduced to LES in order to incorporate unaccounted effects from below the grid-scale, and to improve the quality of the simulation at lower computational cost. We measured the quality of the SGS models by their capability to reproduce the results of a reference simulation at higher resolution. The reference quantities included the energy spectra, the evolution of different statistical moments of the kinetic and magnetic energies, the vorticity, the current density and the dilatation magnitudes. In total, we compared three SGS models: eddy-viscosity, scalesimilarity and nonlinear. Additionaly, we evaluated the influence of using implicit-, grid-filtered quantities versus explicitly filtered quantities to calculate the model terms. We analyzed an ensemble of 19 different initial conditions for each configuration as temporary, transient fluctuations can easily dominate individual simulations.

We find that the simulations employing a grid-filtered eddy-viscosity (EV-GS) or nonlinear (NL-GS) model, or a scale-similarity model with the tested explicit filters (SS-F3 and SS-F5) produce results that are indistinguishable from an implicit LES, i.e. without an explicit model, at the same resolution. Moreover, we find that the eddyviscosity and nonlinear models with the two tested explicit filter widths, i.e. with filter widths of $2.71 \Delta_{x}$ (EVF3 and NL-F3) and $4.75 \Delta_{x}$ (EV-F5 and NL-F5), introduce little additional dissipation on the smallest represented scales e.g. in the energy spectra or the evolu- tion of the mean quantities. Finally, the nonlinear model (NL-F3 and NL-F5) improves higher order statistics of small-scale dependent quantities, such as the kurtosis and skewness of the current density, dilatation and vorticity. For these quantities, the results of an ILES at doubled resolution (in each dimension) can be achieved while introducing only a small computational overhead - less than factor of 1.5 (versus $\approx 16$ for the higher-resolution ILES). This similarly applies to the normalized structure functions and is independent of the explicit filter width.

Based on these results we conclude that an explicit filter is required in order to obtain a measurable impact of an SGS model for shock-capturing finite-volume schemes of second order. In how far this conclusion holds for schemes of higher order and more dynamic versions of the SGS models, e.g. with dynamic coefficients, is yet to be seen and subject to future work. Furthermore, additional dissipation for the explicitly filtered models is not required as numerical dissipation proves to be sufficient (if not too high). Thus, the introduction of an eddy-viscosity model in these schemes is unnecessary. However, as the nonlinear model improves higher order statistics, it would be desirable to remove the unnecessary dissipation to improve the lower-order statistics as well. This kind of regularization is also subject of future work. Finally, the nonlinear model in its current version can readily be used with a small explicit filter in situation where higher order statistics are important at little extra cost. The associated code will be made publicly available together with the publication.

\section{ACKNOWLEDGMENTS}

PG acknowledges financial support by the International Max Planck Research School for Solar System Science at the University of Göttingen. DV acknowledge research funding by the Max-Planck-Institut für Dynamik und Selbstorganisation. DRGS thanks for funding through Fondecyt regular (project code 1161247) and through the "Concurso Proyectos Internacionales de Investigación, Convocatoria 2015" (project code PII20150171). The ENZO simulations were performed and analyzed with the HLRN-III facilities of the NorthGerman Supercomputing Alliance under grant nip0003\%.
[1] S. A. Balbus and J. F. Hawley, Rev. Mod. Phys. 70, (1998).

[2] M. L. Goldstein, D. A. Roberts, and W. H. Matthaeus, ARA\&A 33, 283 (1995).

[3] K. Rodenbeck and D. R. G. Schleicher, Astronomy \& Astrophysics Forthcoming article (2016), 10.1051/0004$6361 / 201527393$.

[4] J. S. Oishi, M.-M. M. Low, D. C. Collins, and M. Tamura, The Astrophysical Journal Letters 806, L12 (2015).
[5] J. Schober, D. R. G. Schleicher, C. Federrath, S. Bovino, and R. S. Klessen, Phys. Rev. E 92, 023010 (2015).

[6] C. M. Cooper, J. Wallace, M. Brookhart, M. Clark, C. Collins, W. X. Ding, K. Flanagan, I. Khalzov, Y. Li, J. Milhone, M. Nornberg, P. Nonn, D. Weisberg, D. G. Whyte, E. Zweibel, and C. B. Forest, Physics of Plasmas 21, 013505 (2014).

[7] P. Tzeferacos, M. Fatenejad, N. Flocke, C. Graziani, G. Gregori, D. Lamb, D. Lee, J. Meinecke, A. Scopatz, and K. Weide, High Energy Density Physics 17, Part A 


\section{Paper IV: Comparative statistics of selected subgrid-scale models in large eddy simulations of decaying, supersonic MHD turbulence}

24 (2015), special Issue: 10th International Conference on High Energy Density Laboratory Astrophysics.

[8] P. Sagaut, Large Eddy Simulation for Incompressible Flows: An Introduction, Scientific Computation (Springer, 2006).

[9] W. Schmidt, Living Reviews in Computational Astrophysics 1 (2015), 10.1007/lrca-2015-2.

[10] D. G. Vlaykov, P. Grete, W. Schmidt, and D. R. G. Schleicher, Physics of Plasmas 23, 062316 (2016), http://dx.doi.org/10.1063/1.4954303.

[11] A. Favre, Physics of Fluids 26, 2851 (1983).

[12] E. Garnier, N. Adams, and P. Sagaut, Large Eddy Simulation for Compressible Flows, Scientific Computation (Springer Netherlands, 2009).

[13] A. A. Chernyshov, K. V. Karelsky, and A. S. Petrosyan, Physics-Uspekhi 57, 421 (2014).

[14] M. Miesch, W. Matthaeus, A. Brandenburg, A. Petrosyan, A. Pouquet, C. Cambon, F. Jenko, D. Uzdensky, J. Stone, S. Tobias, J. Toomre, and M. Velli, Space Science Reviews , 1 (2015).

[15] K. Miki and S. Menon, Physics of Plasmas (1994-present) 15, 072306 (2008)

[16] M. L. Theobald, P. A. Fox, and S. Sofia, Physics of Plasmas 1, 3016 (1994).

[17] W.-C. Müller and D. Carati, Physics of Plasmas (1994present) 9, 824 (2002).

[18] N. Yokoi, Geophysical \& Astrophysical Fluid Dynamics 107, 114 (2013)

[19] F. Grinstein, L. Margolin, and W. Rider, Implicit Large Eddy Simulation: Computing Turbulent Fluid Dynamics (Cambridge University Press, 2007).

[20] P. Grete, D. G. Vlaykov, W. Schmidt, D. R. G. Schleicher, and C. Federrath, New Journal of Physics 17, 023070 (2015)

[21] P. Grete, D. G. Vlaykov, W. Schmidt, and D. R. G. Schleicher, Physics of Plasmas 23, 062317 (2016), http://dx.doi.org/10.1063/1.4954304

[22] J. Smagorinsky, Monthly Weather Review 91, 99 (1963)

[23] B. Vreman, B. Geurts, and H. Kuerten, Journal of Fluid
Mechanics 278, 351 (1994).

[24] J. Bardina, J. Ferziger, and W. Reynolds (American Institute of Aeronautics and Astronautics, 1980).

[25] W. K. Yeo, A generalized high pass/low pass averaging procedure for deriving and solving turbulent flow equations, Ph.D. thesis, The Ohio State University (1987).

[26] G. L. Bryan, M. L. Norman, B. W. O'Shea, T. Abel, J. H. Wise, M. J. Turk, D. R. Reynolds, D. C. Collins, P. Wang, S. W. Skillman, B. Smith, R. P. Harkness, J. Bordner, J. hoon Kim, M. Kuhlen, H. Xu, N. Goldbaum, C. Hummels, A. G. Kritsuk, E. Tasker, S. Skory C. M. Simpson, O. Hahn, J. S. Oishi, G. C. So, F. Zhao, R. Cen, Y. Li, and T. E. Collaboration, The Astrophysical Journal Supplement Series 211, 19 (2014).

[27] O. V. Vasilyev, T. S. Lund, and P. Moin, Journal of Computational Physics 146, 82 (1998).

[28] P. Sagaut and R. Grohens, International Journal for Numerical Methods in Fluids 31, 1195 (1999).

[29] W. Schmidt, C. Federrath, M. Hupp, S. Kern, and J. C. Niemeyer, Astronomy \& Astrophysics 494, 127 (2009).

[30] D. Biskamp, Magnetohydrodynamic Turbulence, by Dieter Biskamp, pp. 310. ISBN 0521810116. Cambridge, UK: Cambridge University Press, September 2003. (Cambrdige University Press, 2003).

[31] R. Benzi, S. Ciliberto, R. Tripiccione, C. Baudet, F. Massaioli, and S. Succi, Phys. Rev. E 48, R29 (1993).

[32] M. Newville, T. Stensitzki, D. B. Allen, and A. Ingargiola, "LMFIT: Non-Linear Least-Square Minimization and Curve-Fitting for Python," (2014).

[33] Z.-S. She and E. Leveque, Phys. Rev. Lett. 72, 336 (1994).

[34] S. Ghosal, Journal of Computational Physics 125, 187 (1996).

[35] F. K. Chow and P. Moin, Journal of Computational Physics 184, 366 (2003).

[36] E. Garnier, M. Mossi, P. Sagaut, P. Comte, and M. Deville, Journal of Computational Physics 153, 273 (1999).

[37] G. S. Winckelmans, A. A. Wray, O. V. Vasilyev, and H. Jeanmart, Physics of Fluids 13, 1385 (2001). 



\section{Summary and conclusions}

In this thesis, we introduced and validated a new nonlinear subgrid-scale model for highly compressible MHD turbulence. Overall, we pursued the following structured approach:

First, we proposed a simple nonlinear closure (Grete et al. 2015) and compared it to the most established closure in literature, i.e. a closure of eddy-viscosity/Smagorinsky type where the local strengths of the turbulent viscosity (and resistivity) are regulated via the SGS energy. The comparison was based on a priori tests in the supersonic regime $\left(\mathrm{M}_{\mathrm{s}} \approx 2-4\right)$ of homogeneous, isotropic, isothermal MHD turbulence. We analyzed the turbulent energy cascade flux across a fixed filter scale and the angular alignment between exact and predicted SGS electromotive force. We found that the simple nonlinear closures for all SGS terms, i.e. kinetic and magnetic SGS stress and EMF, outperform the traditional closures in all tests. They have consistently higher correlations in the energy fluxes, are capable of reproducing the up-scale energy transfer present in the reference data, and show a preferred alignment with the data vector versus random alignment for the eddy-viscosity closures. At the same time, the free coefficients that come with all closures proved to exhibit negligible variations in the case of the nonlinear closures whereas significant variations (and even switching signs) are observed for some coefficients of the eddy-viscosity closure. In addition, we proposed (magnetic) and tested (kinetic and magnetic) instantaneous closures for the SGS energies and showed that both are well correlated with the data maintaining virtually constant coefficients throughout the datasets.

Second, motivated by these promising results, we analytically derived fully compressible nonlinear closures (Vlaykov et al.2016) based on an approximate deconvolution approach via a truncated gradient expansion of the filter kernel. These closures explicitly take into account variations in the density. While the SGS Reynolds stress closure remains unaffected because, as we show, compressibility effects are accounted for by the mass-weighted filtering operation, the closure for the EMF has an additional term. Given that this term is proportional to the partial derivatives of the logarithmic density, it is first expected to be important in the highly supersonic regime where very strong density variations exist. Moreover, we also showed that in the case of mass-weighted filtering the filtering operation and derivation do not commute any more so that additional terms enter both, SGS Reynolds stress and EMF, closures. Eventually, we discussed the energy and cross-helicity dissipation properties of the compressible nonlinear closures and specifically highlighted the potential channels of up-scale transfer, e.g. due to compression via the SGS Maxwell stress.

Third, we largely extended the a priori analysis in Grete et al. (2016) in several ways such as reference quantities, parameter space and closures tested. The reference quantities covered not only the turbulent energy cascade flux, but also turbulent cross-helicity cascade flux, and the total fluxes of both energy and cross-helicity. All these quantities 
evaluate the functional performance - as does the average (i.e. global) SGS dissipation, which we analyzed, too. In addition, we also assessed the structural performance of different closures, in particular, the topological structure of the SGS stresses, and the alignment and magnitude of the SGS vectors in the primary equations. All tests were performed over a parameter space now ranging from the weakly compressible $\left(\mathrm{M}_{\mathrm{s}} \approx 0.2\right)$ to the highly compressible $\left(\mathrm{M}_{\mathrm{s}} \approx 20\right)$ regime. With respect to the filtering, not only the Gaussian filter was used, but also the box filter. Furthermore, the filter scale was varied significantly so that it covered the full range from close to the forcing scale up to the beginning of the (numerically) dissipative scales. Lastly, the amount of tested closures has been greatly increases. Specifically, closures of scale-similarity type were tested next to eddy-viscosity and nonlinear type closures. For the latter two types, also scalings based on different SGS quantities that locally determine the strength of the overall closure were evaluated, including SGS energy and SGS cross-helicity based ones. We found that across all reference quantities and parameters the nonlinear closures outperform closures based on the other two approaches. In particular, we were able to show that the explicit compressible extension of the nonlinear closures significantly increases the correlation between closure and reference data when compared to the simple compressible version in the highly supersonic regime. Even more, the original (unscaled) nonlinear closures do not require an external coefficient as the optimal coefficient (from nonlinear least-square fitting) was found to be unity.

Fourth and finally, we implemented the best performing closure of each type (eddyviscosity, scale-similarity, and nonlinear) in an existing shock-capturing finite-volume code and analyzed their performance as an SGS model in large eddy simulation of decaying, supersonic MHD turbulence (Grete et al. submitted). The test setup was comprised of an ensemble of 19 different realizations in which for each we followed the decay from an initial sonic Mach number of $\mathrm{M}_{\mathrm{s}} \approx 3$ for all models. Moreover, we implemented an explicit filtering operation and varied the effective scale on which the SGS models were calculated from using the grid-scale ( $\Delta_{x}$, no explicit filter) to calculations based quantities subject to an explicit 5-point stencil filter $\approx 5 \Delta_{x}$. We then compared the evolution against the results from (no model) implicit LES at the same and higher resolution. We found that lower-order statistics, e.g. the mean magnitudes of kinetic energy, magnetic energy, vorticity, current and dilatation are mostly dominated by resolution effects and that the presence of an SGS model has virtually no influence on the flow. This is also true for the higher-order statistics (e.g. skewness and kurtosis) if the SGS models are calculated at the grid-scale, or in case of the scale-similarity model even in general. In contrast to this, the nonlinear SGS model employing an explicit filter is able to match the performance of the reference run at twice the resolution (in each dimension), for instance for derived quantities such as the current density and the vorticity magnitude. We also showed that the improvement is present in the longitudinal velocity structure functions. Finally, we concluded that, despite the odds of being masked by the numerical dissipation in shock-capturing methods, the presence of an SGS model (in particular the proposed nonlinear one) improves higher-order statistics, which are essential in our understanding of turbulence.

Related work to the subject of subgrid-scale modeling of (highly) compressible MHD turbulence in finite-volume methods is rather sparse, or, more precisely, non-existent to 
our knowledge. However, different aspects have been covered by different groups.

One of the most active groups with approximately 10 papers since 2006 is probably Chernyshov et al. (2014). The latter is a review covering their previous work. In general, they employ a compressible framework to analyze mostly decaying, e.g. Chernyshov et al. (2006, 2007, 2009) MHD turbulence. Nevertheless, their simulations typically cover the weakly compressible and sub-Alfvénic regime only. Thus, they can employ a highorder finite-difference scheme and forgo shock-capturing mechanisms. Similar to our work, they evaluate different SGS models including models of eddy-viscosity type (with different scalings) and of scale-similarity type. However, despite using a compressible formalism they always neglect the SGS Maxwell stress referring to Müller and Carati (2002), who find that it has no measurable impact on the results of forced and decaying incompressible MHD turbulence using a pseudospectral code. In general, Chernyshov et al. (2014) find that the Smagorinsky-type eddy-viscosity model with a dynamic calculation of the coefficients (Germano et al. 1991, Lilly 1992) and a cross-helicity based model provide the best results when comparing LES (at a resolution of $64^{3}$ ) to DNS (at a resolution of at most $256^{3}$ ). In light of their numerical scheme this is expected. Given that high-order finite-difference schemes usually have very low numerical dissipation any SGS model providing additional mechanisms to dissipate energy is expected to improve the quality of the solution.

Similar findings are reported by Miki and Menon (2008), who use a finite volume code based on central differences. Again, they employ a compressible formalism in the derivation of the closures, but eventually apply them in the incompressible limit (of forced, decaying and rotating turbulence). In contrast to the previous works, they only use single closure strategy, which, in turn, is much more elaborate. In particular, they build upon the early work of Yoshizawa (1990), who used a two-scale direct interaction approximation to derive closures for all terms. Both SGS stresses are modeled with diffusive terms, i.e. a Smagorinsky-type eddy-viscosity for the kinetic part and an equivalent eddy-diffusivity for the magnetic part. The electromotive force contains three terms: an $\alpha$ term proportional to the residual helicity, a $\beta$ term corresponding to an eddy-resistivity, and a $\gamma$ term incorporating SGS cross-helicity. In addition, Miki and Menon (2008) introduce dynamical equations for the kinetic and magnetic SGS energies, which are also used to locally determine the strengths of the diffusive SGS terms. A similar framework is also used by Yokoi (2013) but for incompressible mean-field simulations. In comparison to our results, we were not able to confirm any particular significance of the eddy-diffusivity closure for the SGS Maxwell stress or the cross-helicity related term in the EMF a priori. In fact, independent of the scaling used for the stress tensor (e.g. based on cross-helicity or energy), closure and reference data are virtually uncorrelated, and, in the case of the EMF, the additional cross-helicity term does not improve the moderate correlations already provided by an eddy-resistivity term.

This is also in agreement with Müller and Carati (2002), who, as already mentioned, measure no impact by these terms a posteriori. More generally, they compare the results from DNS of forced (at a resolution of $256^{3}$ ) and decaying $\left(512^{3}\right.$ ) helical MHD turbulence to LES $\left(64^{3}\right)$ with different SGS models including a dynamic eddy-viscosity model, a cross-helicity based model, a model of nonlinear type, and mixed variants of these. Given their low-dissipation pseudospectral scheme they find that basically all models improve the low resolution LES by providing an additional dissipation mechanism. However, one 
model, the cross-helicity based, model is observed to "perform outstandingly well". This is attributed to the fact that, by allowing for a negative eddy-resistivity in the EMF closure, magnetic energy can be transferred to large scales effectively supporting the inverse cascade of magnetic helicity.

While all the results mentioned so far are only concerned with a posteriori performance of MHD SGS models, there also exists (to our knowledge) a single work related to $a$ priori verification. Balarac et al. (2010) analyze eight different EMF models, including eddy-resistivity, scale-similarity, nonlinear and mixed models, in an incompressible, forced, homogeneous, isotropic MHD turbulence simulation using a pseudospectral code at a resolution of $256^{3}$. Similar to our work, they use different filter types (here, spectral and box) and scales, and differentiate between functional and structural performance. They find that structural models, i.e. of scale-similarity and nonlinear type, provide better results than functional ones with the mixed scale-similarity model (complemented by an eddy-resistivity term) showing overall the best performance. While their functional analysis is based on the turbulent energy cascade flux (as is our first investigation), their structural evaluation makes use of optimal estimator theory. Moreau et al. (2006) generally proposed this concept to a priori verification of LES. To be more specific, an artificial neural network (ANN) is used (i.e. trained) to provide the best approximation to an observable variable (e.g. the EMF) given a limited set of input variables (e.g. the filtered quantities). Just recently, Gamahara and Hattori (2016) employed this method to study models for the SGS Reynolds stress in incompressible hydrodynamics. The ANN does initially not possess any structure as it is first constructed during the training phase. Interestingly, they find that the final ANN after the training closely resembles a nonlinear type SGS model in terms of structure and performance. This is also in agreement with the findings of a project (in progress) of ours in which we briefly evaluated MHD SGS closures with ANNs.

Main conclusions The majority of the related work covers the incompressible or weakly compressible MHD regime. However, supersonic shocks and magnetic fields are present in many different astrophysical systems, from the solar wind over the interstellar medium to galaxy clusters. Given their compressible nature and their strong influence on the overall dissipative behavior of a system, compressible effects should not be neglected easily. Instead, an explicit and correct treatment is often crucial to the representation of such physical system in a simulation. The new nonlinear closures explicitly take compressibility into account. We also showed that it significantly improves the SGS model performance in the highly supersonic regime. Thus, large eddy simulations of, for example, the molecular clouds in ISM with Mach numbers $M_{s} \gtrsim 10$ should benefit from this explicit treatment.

Turbulent pressure contributes a significant fraction to the total pressure in many systems. In the presence of magnetic fields, the turbulent pressure is not only of hydrodynamic nature any more, but there is turbulent magnetic pressure, too. Again, omitting this feature potentially translates to omitting a relevant physical ingredient in the overall dynamics. The nonlinear closure automatically accounts for the unresolved magnetic pressure and no special treatment is required. Hence, LES with the nonlinear SGS model could, for instance, contribute to the discussion on the role of the negative effective magnetic pressure instability (NEMPI, Brandenburg et al. 2011). This instability is driven by 
the imbalance between the kinetic and magnetic contributions to the total turbulent energy and turbulent pressure. It allows for the large-scale concentration of coherent magnetic fields from small-scale motion and thus might drive the formation of solar active regions.

Finally, dynamo processes are present on many different scales in the universe. In the process, kinetic energy is converted to magnetic energy. However, the process is not necessarily local in the sense that magnetic energy can grow on the large scales from turbulent motions on the smallest scales (cf. the small-scale dynamo). Naturally, the nonlinear model allows for energy transfer from the unresolved to the resolved scales. For this reason, the known resolution dependence of small-scale dynamo action could possibly alleviated by introducing the nonlinear SGS model to the simulations.

Outlook As we were able to show in the previous chapter, SGS models, in fact, work in shock-capturing finite volume methods when explicit filtering is used. In particular, the nonlinear model is able to improve higher-order statistics. However, it also slightly increases the dissipation on the smallest scales. Generally, this behavior is unfavorable in shock-capturing methods given that the numerical dissipation should be kept to a minimum in order to achieve the highest accuracy possible. An optimal shock-capturing scheme thus provides this minimum only. For this reason, regularization of the nonlinear model could be introduced when applied in shock-capturing schemes targeting, for example, highly supersonic astrophysical flows. The discussion would, of course, be different for other schemes such as spectral schemes or higher-order compact central ones. In the latter case, Vreman et al. (1997) suggested to limit the excessive backscatter of nonlinear type models by using the model locally only in places where it produces down-scale energy transfer. This idea is based on the observation and a similar proposition for the scale-similarity model by Liu et al. (1994), which is also known to produce excessive backscatter. More recently, this approach has been refined by Vollant et al. (2016) who split the flux according to compressional, stretching and rotational effects and eventually suppress the component associated to inverse transfer only. This concept could be transferred to shock-capturing schemes with a fundamental difference: suppressing the downscale transfer rather than the backscatter. Assuming that the shock-capturing provides just the necessary amount of dissipation, the nonlinear model would then be able to provide a channel for up-scale transfer (of e.g. magnetic energy) while not introducing additional dissipation at the same time. An alternative approach to reduce the excess dissipation of the nonlinear model could be the introduction of a mixed model, i.e. the nonlinear model is complemented with an eddy-viscosity term. In contrast to non-shock-capturing schemes, where this type of mixed model is introduced to stabilize the backscatter of structural models (Bardina et al. 1983), here, a negative eddy-viscosity could be used to counteract the dissipative effect of structural models. However, we point out that this kind of mixed model has no physical grounds and would be motivated from a purely numerical point of view.

Another potential improvement of the overall implementation in practice concerns the explicit filtering. Given that this type of filter is essential (grid-scale based models have no significant influence on the evolution of the flow) an efficient calculation is desirable. Due to the nature of being a convolution, this is straightforward in spectral space, whereas the procedure is much more inefficient in real space implementations, e.g., because of distant memory access. In astrophysical simulations adaptive mesh refinement (AMR) (Berger 
and Colella 1989) is often used to bridge the gap between the vastly varying scales. In AMR, regions of interest are simulated at a higher resolution than the root grid. However, the quantities, which are calculated on fine grids, are also present on the coarser grid and already in a smoothed, averaged fashion. These quantities would naturally correspond to the low-pass filtered representations of the quantities calculated on the smaller scales. Unfortunately, this kind of implicit filtering is anisotropic by construction. A cell on the coarse grid hosts $2^{3}$ finer cells (in the case of a 3-dimensional simulation and a refinement factor of 2) of which none is spatially centered with respect to the higher level. In how far this influences the integrated results can hardly be estimated a priori. For this reason, we suggest a more detailed study on the connection between explicit filtering and AMR.

There also exists an interesting extension of the overall formalism. The deconvolution approach we used to derive the compressible nonlinear model straightforwardly extends to closure terms of higher order. Thus, it could also be used to model the unclosed terms in the dynamic equations of e.g. SGS kinetic and magnetic energy, or SGS cross-helicity. In contrast to the zero-equation closures we derived, these additional equations would allow for an intermediate reservoir to mediate between different (additional) SGS physics.

In astrophysics, this technique is used by Schmidt et al. (2014) to explicitly treat the commutator between grids of different resolutions in AMR, or by Braun and Schmidt (2015) to take non-adiabatic processes in the multi-phase ISM into account. While this treatment is so far only applied in hydrodynamic simulations, it offers great potential in MHD applications, too. A more detailed representation of subgrid-scale physics could significantly improve the quality of simulations, especially in processes where the interaction between (unresolved or small-scale) kinetic and magnetic fields is important. This concerns, for example, simulations involving a dynamo, the redistribution of magnetic fields from supernovae, or the joint dynamics of turbulent motion and small-scale magnetic fields during galaxy mergers (Rodenbeck, Kai and Schleicher, Dominik R. G. 2016). Similarly, Egan et al. (2016) presented a first step towards a more detailed, coherent description of subgrid-scale physics in the ICM. The local plasma properties and in particular the interaction of plasma turbulence and magnetic fields should eventually be incorporated in an SGS model on the macroscopic scales. This model aims at treating different physical processes including thermal conduction, viscosity and turbulence as intrinsically linked below the grid scale. Thus, a uniform description, i.e. a self-consistent SGS model from first principles, is desirable.

We conclude that this work provides a solid basis from which it can be expanded into many different areas: from a theoretical point of view connected to the numerical scheme, over practical aspects concerning the implementation, to actual simulations of a wide variety of (astrophysical) phenomena including stellar convection, the ISM and galaxy cluster dynamics - and all this despite being barely able to simulate the simple cup of coffee discussed at the very beginning. 


\section{Bibliography}

F. Anselmet, Y. Gagne, E. J. Hopfinger, and R. A. Antonia. High-order velocity structure functions in turbulent shear flows. Journal of Fluid Mechanics, 140:63-89, 1984. doi: $10.1017 /$ S0022112084000513.

G. Balarac, A. G. Kosovichev, O. Brugière, A. A. Wray, and N. N. Mansour. Modeling of the subgrid-scale term of the filtered magnetic field transport equation. In Proceedings of the Summer Program, pages 503-512, Center for Turbulence Research, Stanford University/NASA, 2010.

Dinshaw S. Balsara, Tobias Rumpf, Michael Dumbser, and Claus-Dieter Munz. Efficient, high accuracy ader-weno schemes for hydrodynamics and divergence-free magnetohydrodynamics. J. Comput. Phys., 228(7):2480-2516, 2009. doi: 10.1016/j.jcp.2008.12. 003.

J. Bardina, J. Ferziger, and W. Reynolds. Improved subgrid-scale models for large-eddy simulation. American Institute of Aeronautics and Astronautics, 1980. doi: 10.2514/6. 1980-1357.

J Bardina, JH Ferziger, and WC Reynolds. Improved turbulence models based on large eddy simulation of homogeneous, incompressible turbulent flows. Stanford Univ. Report, 1(TF-19), 1983.

R. Benzi, S. Ciliberto, R. Tripiccione, C. Baudet, F. Massaioli, and S. Succi. Extended self-similarity in turbulent flows. Phys. Rev. E, 48:R29-R32, 1993. doi: 10.1103/ PhysRevE.48.R29.

M.J. Berger and P. Colella. Local adaptive mesh refinement for shock hydrodynamics. Journal of Computational Physics, 82(1):64 - 84, 1989. doi: 10.1016/0021-9991(89) 90035-1.

Dieter Biskamp. Magnetohydrodynamic Turbulence. Cambrdige University Press, 2003.

S. I. Braginskii. Transport Processes in a Plasma. Reviews of Plasma Physics, 1:205, 1965.

Axel Brandenburg and Kandaswamy Subramanian. Astrophysical magnetic fields and nonlinear dynamo theory. Physics Reports, 417(1-4):1 - 209, 2005. doi: 10.1016/j. physrep.2005.06.005. 
Axel Brandenburg, Koen Kemel, Nathan Kleeorin, Dhrubaditya Mitra, and Igor Rogachevskii. Detection of negative effective magnetic pressure instability in turbulence simulations. The Astrophysical Journal Letters, 740(2):L50, 2011.

H. Braun and W. Schmidt. The small and the beautiful: how the star formation law affects galactic disc structure. Monthly Notices of the Royal Astronomical Society, 454(2): 1545-1555, 2015. doi: 10.1093/mnras/stv1856.

M. Brüggen and F. Vazza. Turbulence in the Intracluster Medium, pages 599614. Springer Berlin Heidelberg, Berlin, Heidelberg, 2015. doi: 10.1007/ 978-3-662-44625-6_21.

G. Brunetti and T. W. Jones. Cosmic Rays in Galaxy Clusters and Their Interaction with Magnetic Fields, pages 557-598. Springer Berlin Heidelberg, Berlin, Heidelberg, 2015. doi: 10.1007/978-3-662-44625-6_20.

Greg L. Bryan, Michael L. Norman, Brian W. O’Shea, Tom Abel, John H. Wise, Matthew J. Turk, Daniel R. Reynolds, David C. Collins, Peng Wang, Samuel W. Skillman, Britton Smith, Robert P. Harkness, James Bordner, Ji hoon Kim, Michael Kuhlen, Hao Xu, Nathan Goldbaum, Cameron Hummels, Alexei G. Kritsuk, Elizabeth Tasker, Stephen Skory, Christine M. Simpson, Oliver Hahn, Jeffrey S. Oishi, Geoffrey C. So, Fen Zhao, Renyue Cen, Yuan Li, and The Enzo Collaboration. Enzo: An adaptive mesh refinement code for astrophysics. The Astrophysical Journal Supplement Series, 211 (2):19, 2014.

C. Canuto, M.Y. Hussaini, A.M. Quarteroni, and J.Z. Thomas A. Spectral Methods in Fluid Dynamics. Springer Series in Computational Physics. Springer Berlin Heidelberg, 1988. doi: 10.1007/978-3-642-84108-8.

V. M. Canuto and J. Christensen-Dalsgaard. Turbulence in astrophysics: Stars. Annual Review of Fluid Mechanics, 30(1):167-198, 1998. doi: 10.1146/annurev.fluid.30.1. 167.

A. A. Chernyshov, K. V. Karelsky, and A. S. Petrosyan. Large-eddy simulation of magnetohydrodynamic turbulence in compressible fluid. Physics of Plasmas, 13(3):032304, 2006. doi: 10.1063/1.2171705.

A. A. Chernyshov, K. V. Karelsky, and A. S. Petrosyan. Development of large eddy simulation for modeling of decaying compressible magnetohydrodynamic turbulence. Physics of Fluids (1994-present), 19(5):055106, 2007. doi: 10.1063/1.2728936.

A A Chernyshov, K V Karelsky, and A S Petrosyan. Subgrid-scale modeling for the study of compressible magnetohydrodynamic turbulence in space plasmas. Physics-Uspekhi, 57(5):421-452, 2014. doi: 10.3367/UFNe.0184.201405a.0457.

Alexander A. Chernyshov, Kirill V. Karelsky, and Arakel S. Petrosyan. Validation of large eddy simulation method for study of flatness and skewness of decaying compressible magnetohydrodynamic turbulence. Theoretical and Computational Fluid Dynamics, 23(6):451-470, 2009. doi: 10.1007/s00162-009-0153-2. 
A.R. Choudhuri. The Physics of Fluids and Plasmas: An Introduction for Astrophysicists. Cambridge University Press, 1998.

Robert A. Clark, Joel H. Ferziger, and W. C. Reynolds. Evaluation of subgrid-scale models using an accurately simulated turbulent flow. Journal of Fluid Mechanics, 91:1-16, 1979. doi: 10.1017/S002211207900001X.

P.A. Davidson. Turbulence: An Introduction for Scientists and Engineers. OUP Oxford, 2004.

A. Dedner, F. Kemm, D. Kröner, C.-D. Munz, T. Schnitzer, and M. Wesenberg. Hyperbolic divergence cleaning for the mhd equations. Journal of Computational Physics, 175(2):645 - 673, 2002. doi: 10.1006/jcph.2001.6961.

H. Egan, B. W. O'Shea, E. Hallman, J. Burns, H. Xu, D. Collins, H. Li, and M. L. Norman. Length Scales and Turbulent Properties of Magnetic Fields in Simulated Galaxy Clusters. ArXiv e-prints, 2016.

C. R. Evans and J. F. Hawley. Simulation of magnetohydrodynamic flows - A constrained transport method. ApJ, 332:659-677, 1988. doi: 10.1086/166684.

Edith Falgarone, Giorgos Momferratos, and Pierre Lesaffre. The Intermittency of ISM Turbulence: What Do the Observations Tell Us?, pages 227-252. Springer Berlin Heidelberg, Berlin, Heidelberg, 2015. doi: 10.1007/978-3-662-44625-6_9.

A. Favre. Turbulence - Space-time statistical properties and behavior in supersonic flows. Physics of Fluids, 26:2851-2863, 1983. doi: 10.1063/1.864049.

C. Federrath, R. S. Klessen, L. Iapichino, and N. J. Hammer. The world's largest turbulence simulations, chapter 1, pages 30-31. Bayerische Akademie der Wissenschaften, 2016.

Christoph Federrath. Inefficient star formation through turbulence, magnetic fields and feedback. Monthly Notices of the Royal Astronomical Society, 450(4):4035-4042, 2015. doi: 10.1093/mnras/stv941.

Joel H. Ferziger and Milovan Perić. Computational Methods for Fluid Dynamics. Springer Berlin Heidelberg, Berlin, Heidelberg, third, rev. edition edition, 2002. Online edition Springer eBook Collection. Engineering.

U. Frisch. Turbulence: The Legacy of AN Kolmogorov. Cambridge University Press, 1995.

B. Fryxell, K. Olson, P. Ricker, F. X. Timmes, M. Zingale, D. Q. Lamb, P. MacNeice, R. Rosner, J. W. Truran, and H. Tufo. FLASH: An Adaptive Mesh Hydrodynamics Code for Modeling Astrophysical Thermonuclear Flashes. Astrophys. J. Suppl. Ser., 131(1):273-334, 2000. doi: 10.1086/317361.

M. Gamahara and Y. Hattori. Searching for turbulence models by artificial neural network. ArXiv e-prints, 2016. 
E. Garnier, N. Adams, and P. Sagaut. Large Eddy Simulation for Compressible Flows. Scientific Computation. Springer Netherlands, 2009. doi: 10.1007/978-90-481-2819-8.

Eric Garnier, Michele Mossi, Pierre Sagaut, Pierre Comte, and Michel Deville. On the use of shock-capturing schemes for large-eddy simulation. Journal of Computational Physics, 153(2):273 - 311, 1999. doi: 10.1006/jcph.1999.6268.

Massimo Germano, Ugo Piomelli, Parviz Moin, and William H. Cabot. A dynamic subgrid-scale eddy viscosity model. Physics of Fluids A, 3(7):1760-1765, 1991. doi: $10.1063 / 1.857955$.

Sergei Konstantinovich Godunov. A difference method for numerical calculation of discontinuous solutions of the equations of hydrodynamics. Matematicheskii Sbornik, 89 (3):271-306, 1959.

P. Goldreich and S. Sridhar. Toward a theory of interstellar turbulence. 2: Strong alfvenic turbulence. ApJ, 438:763-775, 1995. doi: 10.1086/175121.

Philipp Grete, Dimitar G Vlaykov, Wolfram Schmidt, Dominik R G Schleicher, and Christoph Federrath. Nonlinear closures for scale separation in supersonic magnetohydrodynamic turbulence. New Journal of Physics, 17(2):023070, 2015. doi: 10.1088/1367-2630/17/2/023070.

Philipp Grete, Dimitar G. Vlaykov, Wolfram Schmidt, and Dominik R. G. Schleicher. A nonlinear structural subgrid-scale closure for compressible mhd. ii. a priori comparison on turbulence simulation data. Physics of Plasmas, 23(6):062317, 2016. doi: 10.1063/ 1.4954304 .

Philipp Grete, Dimitar G. Vlaykov, Wolfram Schmidt, and Dominik R. G. Schleicher. Comparative statistics of selected subgrid-scale models in large eddy simulations of decaying, supersonic mhd turbulence. submitted.

F.F. Grinstein, L.G. Margolin, and W.J. Rider. Implicit Large Eddy Simulation: Computing Turbulent Fluid Dynamics. Cambridge University Press, 2007.

P. S. Iroshnikov. Turbulence of a Conducting Fluid in a Strong Magnetic Field. Soviet Ast., 7:566, 1964.

A.P. Kazantsev. Enhancement of a magnetic field by a conducting fluid. Sov. Phys. JETP, 26:1031-1034, 1968.

Ralf S. Klessen and Simon C.O. Glover. Physical processes in the interstellar medium. In Yves Revaz, Pascale Jablonka, Romain Teyssier, and Lucio Mayer, editors, Star Formation in Galaxy Evolution: Connecting Numerical Models to Reality, volume 43 of Saas-Fee Advanced Course, pages 85-250. Springer Berlin Heidelberg, 2016. doi: 10.1007/978-3-662-47890-5_2.

A. Kolmogorov. The Local Structure of Turbulence in Incompressible Viscous Fluid for Very Large Reynolds’ Numbers. Akademiia Nauk SSSR Doklady, 30:301-305, 1941. 
A. N. Kolmogorov. A refinement of previous hypotheses concerning the local structure of turbulence in a viscous incompressible fluid at high reynolds number. Journal of Fluid Mechanics, 13:82-85, 1962. doi: 10.1017/S0022112062000518.

Robert H. Kraichnan. Inertial-range spectrum of hydromagnetic turbulence. Physics of Fluids, 8(7):1385-1387, 1965. doi: 10.1063/1.1761412.

Alexei G. Kritsuk, Åke Nordlund, David Collins, Paolo Padoan, Michael L. Norman, Tom Abel, Robi Banerjee, Christoph Federrath, Mario Flock, Dongwook Lee, Pak Shing Li, Wolf-Christian Müller, Romain Teyssier, Sergey D. Ustyugov, Christian Vogel, and Hao Xu. Comparing numerical methods for isothermal magnetized supersonic turbulence. The Astrophysical Journal, 737(1):13, 2011.

A. Leonard. Energy cascade in large-eddy simulations of turbulent fluid flows. In F.N. Frenkiel and R.E. Munn, editors, Turbulent Diffusion in Environmental PollutionProceedings of a Symposium held at Charlottesville, volume 18, Part A of Advances in Geophysics, pages 237 - 248. Elsevier, 1975. doi: 10.1016/S0065-2687(08)60464-1.

R.J. LeVeque. Finite Volume Methods for Hyperbolic Problems. Cambridge Texts in Applied Mathematics. Cambridge University Press, 2002.

D. K. Lilly. A proposed modification of the germano subgrid-scale closure method. Physics of Fluids A, 4(3):633-635, 1992. doi: 10.1063/1.858280.

Shewen Liu, Charles Meneveau, and Joseph Katz. On the properties of similarity subgridscale models as deduced from measurements in a turbulent jet. Journal of Fluid Mechanics, 275:83-119, 1994. doi: 10.1017/S0022112094002296.

TS Lund. On the use of discrete filters for large eddy simulation. Annual Research Briefs, pages 83-95, 1997.

Len G. Margolin and William J. Rider. Numerical regularization: The numerical analysis of implicit subgrid models. In F.F. Grinstein, L.G. Margolin, and W.J. Rider, editors, Implicit Large Eddy Simulation: Computing Turbulent Fluid Dynamics, chapter 5, pages 195-221. Cambridge University Press, 2007.

Mark Miesch, William Matthaeus, Axel Brandenburg, Arakel Petrosyan, Annick Pouquet, Claude Cambon, Frank Jenko, Dmitri Uzdensky, James Stone, Steve Tobias, Juri Toomre, and Marco Velli. Large-eddy simulations of magnetohydrodynamic turbulence in heliophysics and astrophysics. Space Science Reviews, pages 1-41, 2015. doi: 10.1007/s11214-015-0190-7.

Mark S. Miesch. Large-scale dynamics of the convection zone and tachocline. Living Reviews in Solar Physics, 2(1), 2005. doi: 10.1007/lrsp-2005-1.

Kenji Miki and Suresh Menon. Localized dynamic subgrid closure for simulation of magnetohydrodynamic turbulence. Physics of Plasmas (1994-present), 15(7):072306, 2008. doi: 10.1063/1.2947312. 
Takahiro Miyoshi and Kanya Kusano. A multi-state \{HLL\} approximate riemann solver for ideal magnetohydrodynamics. Journal of Computational Physics, 208(1):315 344, 2005. doi: 10.1016/j.jcp.2005.02.017.

A. Moreau, O. Teytaud, and J. P. Bertoglio. Optimal estimation for large-eddy simulation of turbulence and application to the analysis of subgrid models. Physics of Fluids (1994-present), 18(10):105101, 2006. doi: 10.1063/1.2357974.

Wolf-Christian Müller and Daniele Carati. Dynamic gradient-diffusion subgrid models for incompressible magnetohydrodynamic turbulence. Physics of Plasmas (1994present), 9(3):824-834, 2002. doi: 10.1063/1.1448498.

A. M. Obukhov. Some specific features of atmospheric turbulence. Journal of Geophysical Research, 67(8):3011-3014, 1962. doi: 10.1029/JZ067i008p03011.

Stephen B. Pope. Turbulent Flows. Cambridge University Press, 2000. Cambridge Books Online.

L. F. Richardson. Weather Prediction by Numerical Process. Cambridge University Press, Cambridge, 1922.

Rodenbeck, Kai and Schleicher, Dominik R. G. Magnetic fields during galaxy mergers. AEFA, 593:A89, 2016. doi: 10.1051/0004-6361/201527393.

P. Sagaut. Large Eddy Simulation for Incompressible Flows: An Introduction. Scientific Computation. Springer, 2006.

P. Sagaut and R. Grohens. Discrete filters for large eddy simulation. International Journal for Numerical Methods in Fluids, 31(8):1195-1220, 1999. doi: 10.1002/(SICI) 1097-0363(19991230)31:8<1195::AID-FLD914>3.0.CO;2-H.

W Schmidt and C Federrath. A fluid-dynamical subgrid scale model for highly compressible astrophysical turbulence. Astron. Astrophys., 528:A106, 2011. doi: 10.1051/ 0004-6361/201015630.

W. Schmidt, C. Federrath, M. Hupp, S. Kern, and J. C. Niemeyer. Numerical simulations of compressively driven interstellar turbulence. I. Isothermal gas. Astronomy $\mathcal{E}$ Astrophysics, 494:127-145, 2009. doi: 10.1051/0004-6361:200809967.

W. Schmidt, A. S. Almgren, H. Braun, J. F. Engels, J. C. Niemeyer, J. Schulz, R. R. Mekuria, A. J. Aspden, and J. B. Bell. Cosmological fluid mechanics with adaptively refined large eddy simulations. MNRAS, 440:3051-3077, 2014. doi: 10.1093/mnras/ stu501.

Wolfram Schmidt. Turbulent Thermonuclear Combustion in Degenerate Stars. Dissertation, Technische Universität München, München, 2004.

Wolfram Schmidt. Large eddy simulations in astrophysics. Living Reviews in Computational Astrophysics, 1(2), 2015. doi: 10.1007/lrca-2015-2. 
Zhen-Su She and Emmanuel Leveque. Universal scaling laws in fully developed turbulence. Phys. Rev. Lett., 72:336-339, 1994. doi: 10.1103/PhysRevLett.72.336.

Frank H. Shu, Fred C. Adams, and Susana Lizano. Star formation in molecular clouds: Observation and theory. Annual Review of Astronomy and Astrophysics, 25(1):23-81, 1987. doi: 10.1146/annurev.aa.25.090187.000323.

J. Smagorinsky. General Circulation Experiments with the Primitive Equations. Monthly Weather Review, 91:99, 1963. doi: 10.1175/1520-0493(1963)091<0099:GCEWTP>2. 3.CO;2.

Volker Springel. The cosmological simulation code gadget-2. Monthly Notices of the Royal Astronomical Society, 364(4):1105-1134, 2005. doi: 10.1111/j.1365-2966.2005. 09655.x.

S. Stolz and N. A. Adams. An approximate deconvolution procedure for large-eddy simulation. Physics of Fluids, 11(7):1699-1701, 1999. doi: 10.1063/1.869867.

Kandaswamy Subramanian, Anvar Shukurov, and Nils Erland L. Haugen. Evolving turbulence and magnetic fields in galaxy clusters. Monthly Notices of the Royal Astronomical Society, 366(4):1437-1454, 2006. doi: 10.1111/j.1365-2966.2006.09918.x.

S. M. Tobias, F. Cattaneo, and S. Boldyrev. MHD Dynamos and Turbulence. In P.A. Davidson, Y. Kaneda, and K.R. Sreenivasan, editors, Ten Chapters in Turbulence, chapter 9, pages 351-404. Cambridge University Press, 2013.

Eleuterio F Toro. Riemann solvers and numerical methods for fluid dynamics: a practical introduction. Springer Science \& Business Media, 2009.

Oleg V. Vasilyev, Thomas S. Lund, and Parviz Moin. A general class of commutative filters for $\{$ LES $\}$ in complex geometries. Journal of Computational Physics, 146(1):82 - 104, 1998. doi: 10.1006/jcph.1998.6060.

Enrique Vázquez-Semadeni. Interstellar MHD Turbulence and Star Formation, pages 401-444. Springer Berlin Heidelberg, Berlin, Heidelberg, 2015. doi: 10.1007/ 978-3-662-44625-6_14.

Dimitar G Vlaykov. Sub-grid Scale Modelling of Compressible Magnetohydrodynamic Turbulence: Derivation and A Priori Analysis. PhD thesis, Georg-August University School of Science (GAUSS) Göttingen, 2015.

Dimitar G. Vlaykov, Philipp Grete, Wolfram Schmidt, and Dominik R. G. Schleicher. A nonlinear structural subgrid-scale closure for compressible mhd. i. derivation and energy dissipation properties. Physics of Plasmas, 23(6):062316, 2016. doi: 10.1063/ 1.4954303 .

A. Vollant, G. Balarac, and C. Corre. A dynamic regularized gradient model of the subgrid-scale stress tensor for large-eddy simulation. Physics of Fluids, 28(2):025114, 2016. doi: 10.1063/1.4941781. 
A. W. Vreman, B. J. Geurts, J. G. M. Kuerten, and P. J. Zandbergen. A finite volume approach to large eddy simulation of compressible, homogeneous, isotropic, decaying turbulence. International Journal for Numerical Methods in Fluids, 15(7):799-816, 1992. doi: 10.1002/fld.1650150705.

Bert Vreman, Bernard Geurts, and Hans Kuerten. Realizability conditions for the turbulent stress tensor in large-eddy simulation. Journal of Fluid Mechanics, 278:351-362, 1994. doi: 10.1017/S0022112094003745.

Bert Vreman, Bernard Geurts, and Hans Kuerten. Large-eddy simulation of the turbulent mixing layer. Journal of Fluid Mechanics, 339:357-390, 1997. doi: 10.1017/ S0022112097005429.

Peng Wang and Tom Abel. Magnetohydrodynamic simulations of disk galaxy formation: The magnetization of the cold and warm medium. The Astrophysical Journal, 696(1): 96, 2009.

Woon Kwang Yeo. A generalized high pass/low pass averaging procedure for deriving and solving turbulent flow equations. PhD thesis, The Ohio State University, 1987.

N. Yokoi. Cross helicity and related dynamo. Geophysical $\mathcal{E}$ Astrophysical Fluid Dynamics, 107(1-2):114-184, 2013. doi: 10.1080/03091929.2012.754022.

A. Yoshizawa. Self-consistent turbulent dynamo modeling of reversed field pinches and planetary magnetic fields. Physics of Fluids B, 2:1589-1600, 1990. doi: 10.1063/1. 859484.

Yan Zang, Robert L. Street, and Jeffrey R. Koseff. A dynamic mixed subgrid-scale model and its application to turbulent recirculating flows. Physics of Fluids A, 5(12):31863196, 1993. doi: 10.1063/1.858675. 
Appendix 



\section{A Discrete filter approximations}

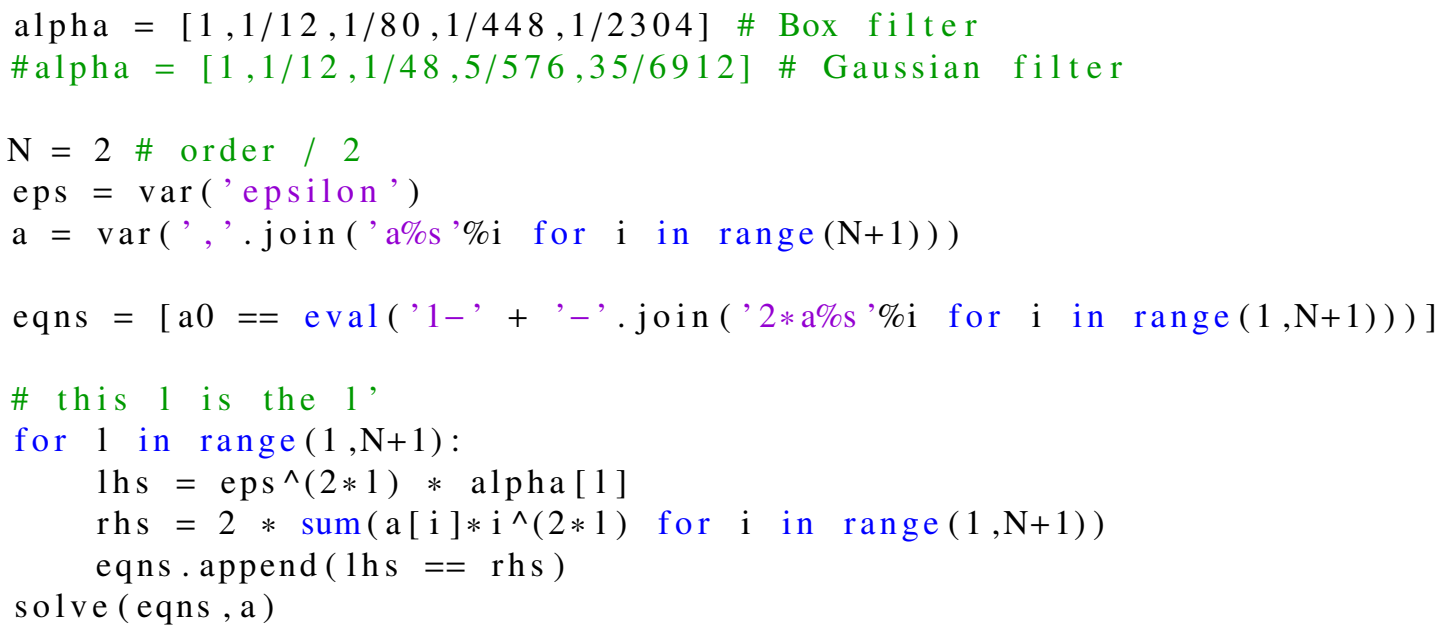

Listing A.1: Code snippet showing the symbolic calculation in SAGE (http:// sagemath.org) of the discrete filter operator coefficients that match the continuous box or Gaussian filter kernel.

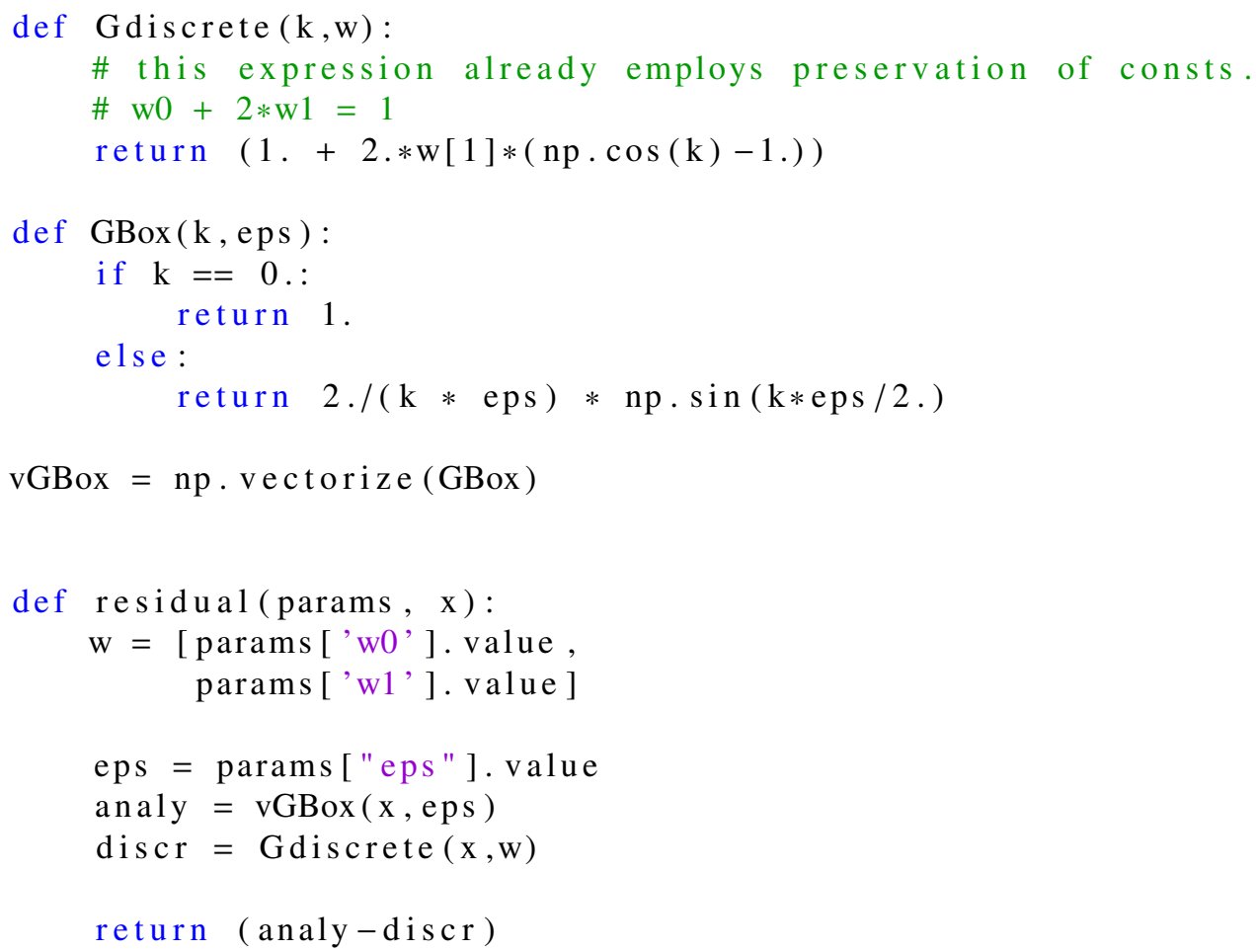


A Discrete filter approximations

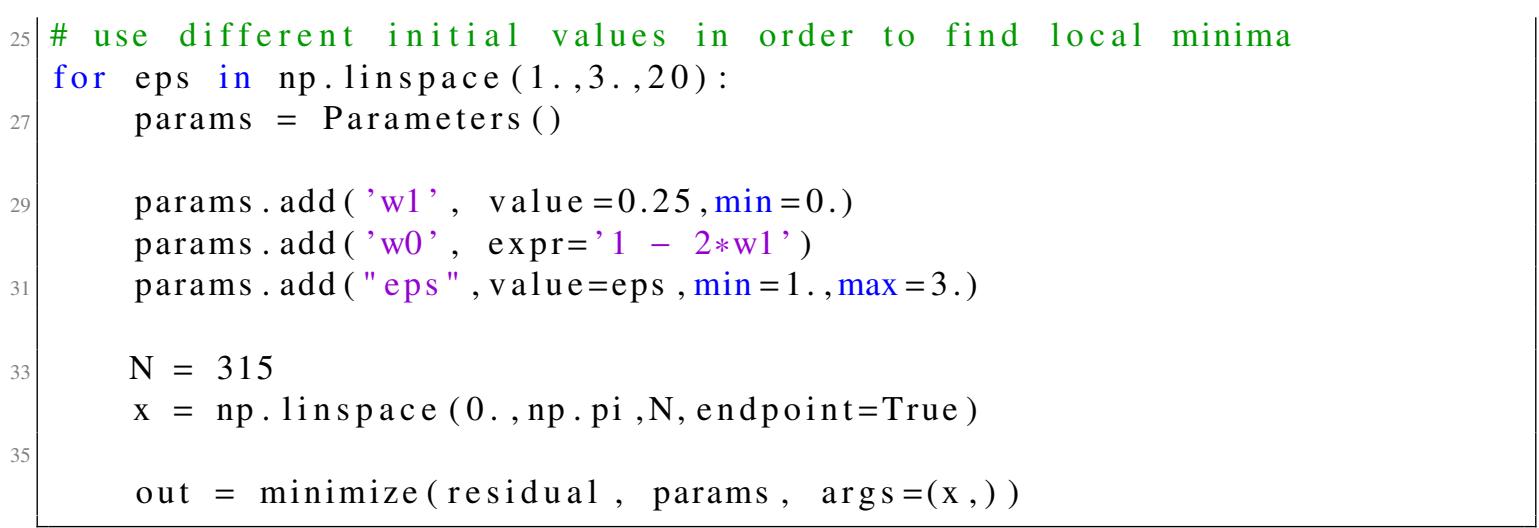

Listing A.2: Python code snippet to calculate optimal coefficients of the discrete box filter to match the continuous filter with the LMFIT package.

Table A.1: Discrete filter coefficient values for a positive, symmetric box filter. The star (*) marks the solution with the lowest residual for a given order in the cases where the coefficients have been determined by least-square fitting to the analytic function.

\begin{tabular}{lccccccc}
\hline ID & Case & order & $\epsilon$ & $a_{0}$ & $a_{1}$ & $a_{2}$ & $a_{3}$ \\
\hline O2-Ia-D2.45 & Ia & 2 & $\sqrt{6}$ & $1 / 2$ & $1 / 4$ & - & - \\
O4-Ia-D4.58 & Ia & 4 & 4.5854 & 0.18886 & 0.25 & 0.15556 & - \\
O6-Ia-D4.58 & Ia & 6 & 4.5848 & 0.18776 & 0.24983 & 0.15612 & 0.00017 \\
O6-Ia-D6.59 & Ia & 6 & 6.5927 & 0.16224 & 0.13932 & 0.16888 & 0.11068 \\
\hline O2-Ib-D2.69 & Ib & 2 & 2.6939 & 0.39524 & 0.30238 & - & - \\
O4-Ib-D2.77 & Ib & 4 & 2.7711 & 0.38438 & 0.30376 & 0.0040484 & - \\
O4-Ib-D4.67 & Ib & 4 & 4.671 & 0.21488 & 0.22038 & 0.17218 & - \\
O6-Ib-D4.77 & Ib & 6 & 4.7733 & 0.20582 & 0.21659 & 0.17834 & 0.0021545 \\
O6-Ib-D6.63 & Ib & 6 & 6.6274 & 0.14217 & 0.15446 & 0.1589 & 0.11556 \\
\hline O2-IIa-D2.71 & IIa & 2 & 2.711 & 0.40149 & 0.29925 & - & - \\
O4-IIa-D2.81* & IIa & 4 & 2.8051 & 0.37989 & 0.30335 & 0.00671 & - \\
O4-IIa-D4.75 & IIa & 4 & 4.7498 & 0.20237 & 0.22208 & 0.17673 & - \\
O6-IIa-D2.81 & IIa & 6 & 2.8051 & 0.37989 & 0.30335 & 0.00671 & 0.00000 \\
O6-IIa-D4.85* & IIa & 6 & 4.8526 & 0.20015 & 0.21362 & 0.18112 & 0.00518 \\
O6-IIa-D6.77 & IIa & 6 & 6.7661 & 0.15192 & 0.14379 & 0.15472 & 0.12555 \\
\hline O2-IIb-D2.60 & IIb & 2 & 2.5978 & 0.43787 & 0.28106 & - & - \\
O4-IIb-D2.69 & IIb & 4 & 2.6926 & 0.40915 & 0.29325 & 0.00222 & - \\
O4-IIb-D4.57* & IIb & 4 & 4.5709 & 0.18768 & 0.25137 & 0.15479 & - \\
O6-IIb-D2.69 & IIb & 6 & 2.6926 & 0.40915 & 0.29320 & 0.00222 & 0.00000 \\
O6-IIb-D4.67 & IIb & 6 & 4.6743 & 0.19559 & 0.23451 & 0.16668 & 0.00101 \\
O6-IIb-D6.55* & IIb & 6 & 6.5461 & 0.18695 & 0.12084 & 0.18130 & 0.10438 \\
\hline
\end{tabular}




\section{Scientific contributions}

\section{Refereed journal articles}

P. Grete, D. G. Vlaykov, W. Schmidt, D. R. G. Schleicher and C. Federrath

Title: "Nonlinear closures for scale separation in supersonic magnetohydrodynamic turbulence"

2015 New J. Phys. 17023070 doi : 10. 1088/1367-2630/17/2/023070

D. G. Vlaykov, P. Grete, W. Schmidt and D. R G Schleicher

Title: "A nonlinear structural subgrid-scale closure for compressible MHD. I. Derivation and energy dissipation properties"

2016 Physics of Plasmas 23062316 doi : 10.1063/1.4954303

P. Grete, D. G. Vlaykov, W. Schmidt and D. R. G. Schleicher

Title: "A nonlinear structural subgrid-scale closure for compressible MHD. II. A priori comparison on turbulence simulation data"

2016 Physics of Plasmas 23062317 doi : 10.1063/1.4954304

P. Grete, D. G. Vlaykov, W. Schmidt and D. R. G. Schleicher

Title: "Comparative statistics of selected subgrid-scale models in large eddy simulations of decaying, supersonic MHD turbulence"

Submitted to a peer-reviewed journal.

\section{Invited Talks}

11/2015 Colloquium talk, Astronomy department, Universidad de Concepción, Chile

Title: "Closing the foodchain of magnetic whorls - Introduction to subgridscale modeling of magnetohydrodynamic turbulence"

06/2015 Philipp Grete and Dimitar Vlaykov

Colloquium talk, Hamburger Sternwarte, Hamburg, Germany

Title: "Nonlinear subgrid-scale closures in magnetohydrodynamic turbulence" 


\section{Conference contributions}

06/2016 Talk at "ASTRONUM 2016 - 11th International Conference on Numerical Modeling of Space Plasma Flows", Monterey, CA, USA

Title: "A compressible, nonlinear subgrid-scale model for magnetohydrodynamic turbulence"

05/2016 | Poster at "6th Les Houches school in numerical physics "International School of Computational Astrophysics", Les Houches, France Title: "Subgrid-scale closures for MHD turbulence"

12/2015 Talk at "16 $6^{\text {th }}$ MHD Days", Ilmenau, Germany

Title: "Subgrid-scale modeling of compressible MHD turbulence"

03/2015 Talk at winter school on "Turbulence, magnetic fields and self organization in laboratory and astrophysical plasmas", Les Houches, France

Title: "Nonlinear subgrid-scale closures in magnetohydrodynamic turbulence"

12/2014 Talk at " $15^{\text {th }}$ MHD Days", Potsdam, Germany

Title: "Nonlinear subgrid-scale closures in magnetohydrodynamic turbulence"

11/2014 Dimitar Vlaykov and Philipp Grete

Poster at Conference "Magnetic fields from the sun to black holes", Paris, France

Title: "Subgrid-scale closures for MHD turbulence"

09/2014 Talk at "XXXIV Dynamics Days Europe, Nonlinear Phenomena in Plasma Astrophysics", Bayreuth, Germany

Title: "Subgrid-scale closures in highly compressible MHD turbulence: turbulent energies"

08/2014 Talk at "5th Black Sea Biennial School and Workshop on Space Plasma Physics", Kiten, Bulgaria

Title: "Subgrid-scale modeling of highly compressible MHD turbulence: momentum closure"

10/2013 Dimitar Vlaykov and Philipp Grete

Poster at workshop on "Astrophysical Turbulence: From Galaxies to Planets", Dresden, Germany

Title: "Large eddy simulations of MHD turbulence" 


\section{Acknowledgements}

First of all, I would like to express my gratitude to my advisers Dominik Schleicher and Wolfram Schmidt. Both possess a unique set of skills, which they happily shared and which allowed for a well-guided path through the $\mathrm{PhD}$ adventure - even from the far north and the other side of the Earth. Similarly, I am deeply grateful to Dimitar Vlaykov, my friend and fellow combatant in $k$-space, for the productive teamwork and inspiring discussions in general. I thank Christoph Federrath for providing the FLAsH datasets we used in the analysis.

I would like to thank Laurent Gizon as a member of my thesis advisory committee, and the members of my examination committee Eberhard Bodenschatz, Marcus Brüggen, Jens Niemeyer, Jörg Büchner and Andreas Dillmann for willingly support and review my thesis. Also, I would like to thank Sonja Schuh for her support in making things happen in an occasionally restrictive environment.

I acknowledge financial support by the International Max Planck Research School for Solar System Science at the University of Göttingen. Moreover, I thank Dominik Schleicher and Jens Niemeyer for additional financial support via the IAG, which allowed me to attend summer and winter schools, and to continue my work at the IAG.

Finally, I am grateful to the colleagues and friends in the cosmology group, in particular Christoph Behrens and Jan Frederik Engels, who made life on floor E exciting and enjoyable, not to mention Kai Rodenbeck without whom the entire floor would starve.

My deepest gratitude goes to my family who never questioned my change of plans and supported me all along the way - not only during the PhD. Last but not least, without the relentless support by Petja this thesis and my mind would be in a very different state. I cannot express how much I value all her many unexpected tokens of appreciation that always make me smile. 